\title{
Synthesis, Structure and Solution Studies of Lithiated Allylic Phosphines and Phosphine Oxides
}

Nimrod M. Eren, Samantha A. Orr, Christopher D. Thompson, Emily C. Border, Michael A. Stevens, and Victoria L. Blair*

School of chemistry, Monash University, Clayton, Melbourne, Victoria 3800 , Australia

\section{Supporting Information}

\section{Contents}

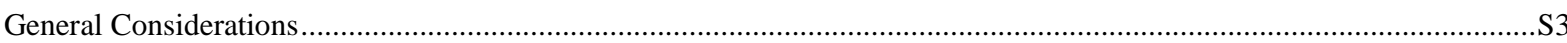

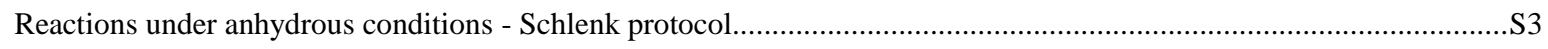

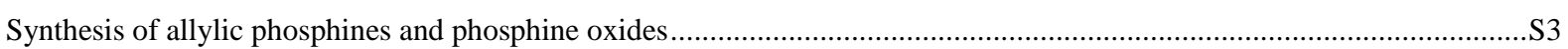

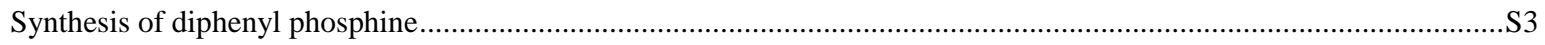

Procedure A: Synthesis of allylic diphenyl phosphines P1, P2 and P3 ...................................................................S3

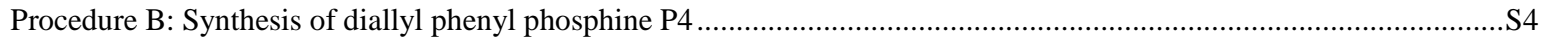

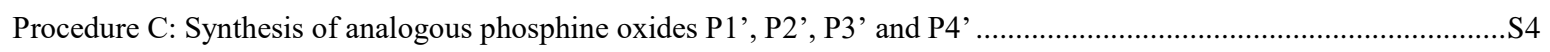

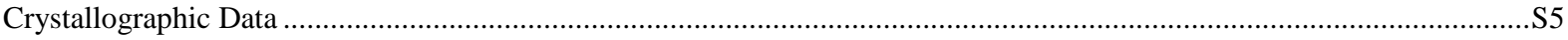

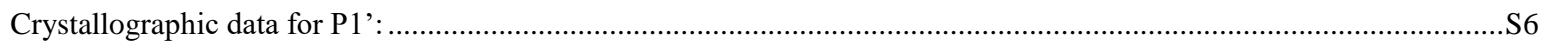

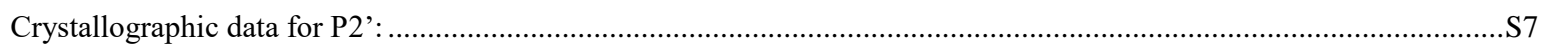

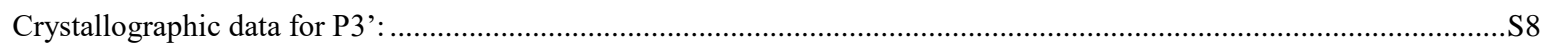

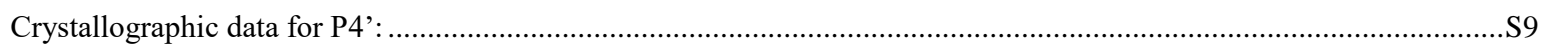

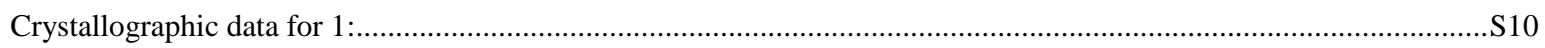

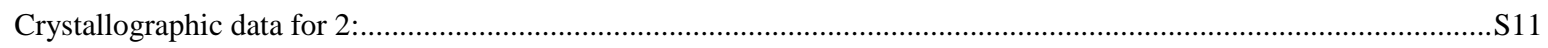

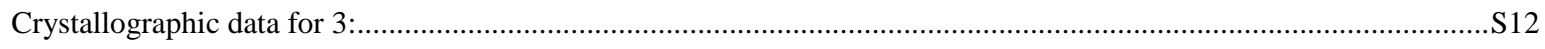

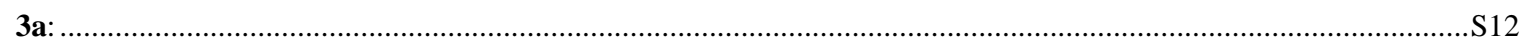

3b:

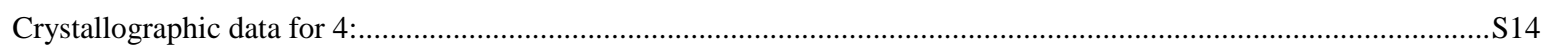

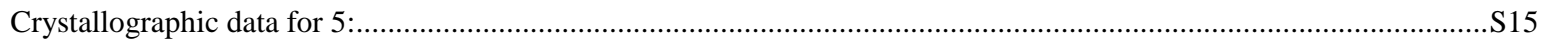

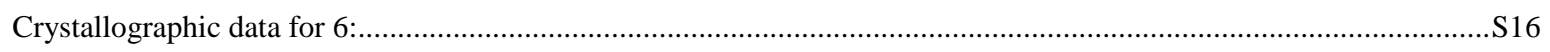

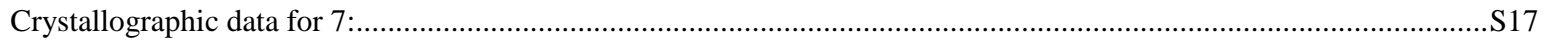

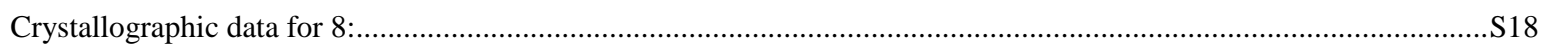

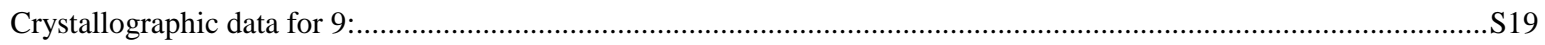

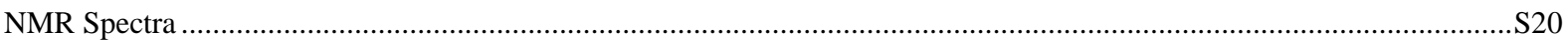

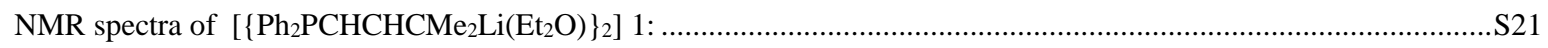

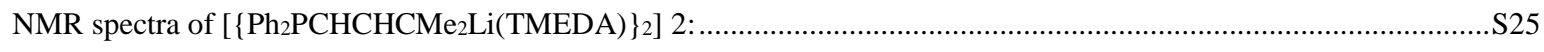

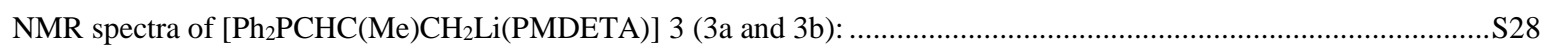

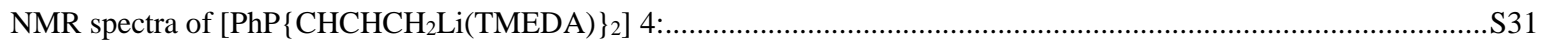

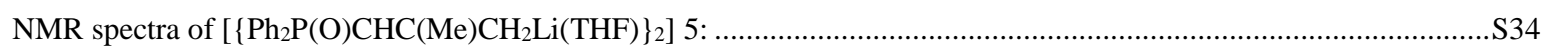

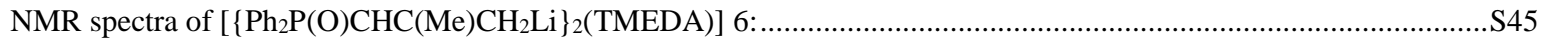

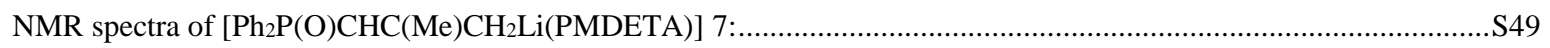


NMR spectra of $\left[\mathrm{Ph}_{2} \mathrm{P}(\mathrm{O}) \mathrm{CHCHCMe}_{2} \mathrm{Li}(\mathrm{PMDETA})\right] 8$

NMR spectra of $\left[\mathrm{Ph}_{2} \mathrm{P}(\mathrm{O}) \mathrm{CHCHCH}_{2} \mathrm{Li}(\mathrm{PMDETA})\right]$ 9:

NMR spectra of $\left[\mathrm{Ph}_{2} \mathrm{PCHCHCMe}_{2} \mathrm{Li}\right.$ (PMDETA) 10 :.

NMR spectra of $\left[\mathrm{Ph}_{2} \mathrm{PCHC}(\mathrm{Me}) \mathrm{CH}_{2} \mathrm{Li}(\mathrm{TMEDA})\right] 11$ : S62

NMR spectra of $\left[\left\{\mathrm{Ph}_{2} \mathrm{P}(\mathrm{O}) \mathrm{CHCHCH}_{2} \mathrm{Li}_{2}\right\}_{2}\left(\mathrm{Et}_{2} \mathrm{O}\right)_{\mathrm{n}}\right] 12$ :

S70 


\section{General Considerations}

\section{Reactions under anhydrous conditions - Schlenk protocol}

All reactions requiring anhydrous conditions were conducted with oven dried glassware under an atmosphere of nitrogen using a vacuum/nitrogen line and Schlenk techniques. All glassware was dried at $105{ }^{\circ} \mathrm{C}$ for approximately 24 hours prior to use and allowed to cool under vacuum to minimise water content. The Schlenk assembly was purged of air and moisture under high vacuum and backfilled with nitrogen three times.

Liquids and solutions of dissolved solids were transferred through rubber seals using oven dried, nitrogen purged syringes or cannulas. Filtering of solutions was carried out through rubber seal using oven dried / nitrogen purged cannula equipped with glass fibre micro filters (GF/A, circles $\emptyset 42.5 \mathrm{~cm}$, Whatman $\left.{ }^{\circledR}\right)$ which were fixed with Teflon rubber.

Air-sensitive compounds were stored in a high purity argon recirculating dry box, with oxygen and moisture levels of $<0.1$ ppm. All analytical samples of air-sensitive compounds were prepared using a high purity argon recirculating dry box.

\section{Synthesis of allylic phosphines and phosphine oxides}

The mono allylic phosphines: allyl diphenyl phosphine, 3,3-dimethylallyl diphenyl phosphine, 2-methylallyl diphenyl phosphine (P1-3) and their oxide derivatives (P'1-3) were synthesised according to the following established procedure:

\section{Synthesis of diphenyl phosphine}<smiles>c1ccc(Pc2ccccc2)cc1</smiles>
Triphenylphosphine (52.45 g, $200.0 \mathrm{mmol})$ and lithim metal (pellets) (5.52 g, $800 \mathrm{mmol})$ were weighed and added into a flask. The flask was then flushed with nitrogen (3x). After the flask was placed in ice, THF $(100 \mathrm{~mL})$ was added dropwise with stirring evolving heat. This reaction was left to stir until it had warmed to room temperature. The solution was filtered into another Schlenk flask and quenched with deoxygenated water $(9 \mathrm{~mL}, 500 \mathrm{mmol})$. The organic layer was transferred into another nitrogen atmosphere Schlenk flask, and the remaining aqueous layer was extracted using Et $2 \mathrm{O}(3 \times 40 \mathrm{~mL})$. The organic phases were then combined, dried with $\mathrm{MgSO}_{4}$, filtered into an $\mathrm{RBF}$ and the solvent was removed in vacuuo. The remaining liquid was then distilled (at ca. 0.15 torr) under vacuum at $80^{\circ} \mathrm{C}$, yielding a clear colourless oil $(20 \mathrm{~mL}, 115 \mathrm{mmol}$, $57 \%$ yield).

\section{Procedure A: Synthesis of allylic diphenyl phosphines P1, P2 and P3}<smiles>C=CCP(c1ccccc1)c1ccccc1</smiles>

P1

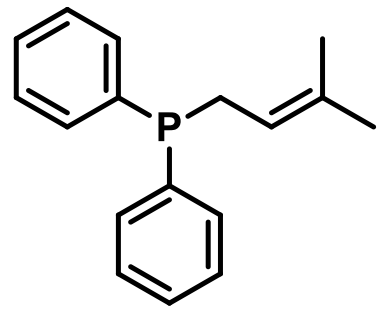

P2<smiles>C=C(C)CP(c1ccccc1)c1ccccc1</smiles>

P3

Diphenylphosphine $(5.0 \mathrm{~mL}, 29$ mmol) was dissolved in $50 \mathrm{~mL}$ of $n$ hexane. $n$-butyllithium $(19 \mathrm{~mL}, 1.6$ M solution in hexane, $30 \mathrm{mmol}$ ) was added dropwise to the diphenylphosphine dropwise at $0{ }^{\circ} \mathrm{C}$ with stirring and reaction was allowed to warm to room temperature overnight. The precipitate was washed with $n$ hexane 3 x $20 \mathrm{~mL}$. The yellow precipitate was then resuspended in $20 \mathrm{~mL} n$-hexane and allylhalide (P1) or 3,3-dimethylallyl halide (P2) or 2-methylallyl halide $(\mathbf{P 3})(31 \mathrm{mmol})$ was added to the solution at $0{ }^{\circ} \mathrm{C}$. The solution was allowed to stir and warm to room temperature overnight. The mixture was filtered using a cannula filter and solvent was removed from the filtrate under vacuum. The remaining colourless oil was purified via vacuum distillation $\left(140-150{ }^{\circ} \mathrm{C}\right.$ at ca. 0.15 torr) as a clear, colourless oil. $60-80 \%$ yield (allyl group dependent).

P1: ${ }^{1} \mathrm{H}$ NMR ( $\left.\mathrm{C}_{6} \mathrm{D}_{6}, 400.20 \mathrm{MHz}\right): \delta 2.71\left(\mathrm{~d},{ }^{3} J_{\mathrm{H}-\mathrm{H}}=7.4 \mathrm{~Hz}, 2 \mathrm{H}, \mathrm{P}-\mathrm{CH}_{2}-\right), 4.91\left(\mathrm{~m}, 2 \mathrm{H},=\mathrm{CH}_{2}\right), 5.77\left(\mathrm{ddtd},{ }^{3} J_{\mathrm{H}-\mathrm{H}(\mathrm{trans})}=17.4\right.$ $\left.\mathrm{Hz},{ }^{3} J_{\mathrm{H}-\mathrm{H}(\mathrm{cis})}=10.2 \mathrm{~Hz},{ }^{3} J_{\mathrm{H}-\mathrm{H} 2}=7.4 \mathrm{~Hz},{ }^{3} J_{\mathrm{H}-\mathrm{P}}=5.8 \mathrm{~Hz}, 1 \mathrm{H},-\mathrm{CH}=\right), 7.07(\mathrm{~m}, 6 \mathrm{H}, m$ - and $p-\mathrm{H}), 7.40(\mathrm{~m}, 4 \mathrm{H}, o-\mathrm{H}) .{ }^{13} \mathrm{C} \mathrm{NMR}$ $\left(\mathrm{C}_{6} \mathrm{D}_{6}, 100.6 \mathrm{MHz}\right): \delta 34.08\left(\mathrm{~d},{ }^{1} J_{\mathrm{C}-\mathrm{P}}=14.9 \mathrm{~Hz}, \mathrm{P}-\mathrm{CH}_{2}-\right), 117.36\left(\mathrm{~d},{ }^{3} J_{\mathrm{C}-\mathrm{P}}=10.5 \mathrm{~Hz},=\mathrm{CH}_{2}\right), 128.64\left(\mathrm{~d},{ }^{3} J_{\mathrm{C}-\mathrm{P}}=6.4 \mathrm{~Hz}, m-\mathrm{C}\right)$, 128.73(s, p-C), $133.26\left(\mathrm{~d},{ }^{1} J_{\mathrm{C}-\mathrm{P}}=18.5 \mathrm{~Hz}, o-\mathrm{C}\right), 133.60\left(\mathrm{~d},{ }^{2} J_{\mathrm{C}-\mathrm{P}}=9.6 \mathrm{~Hz},-\mathrm{CH}=\right), 139.09\left(\mathrm{~d},{ }^{1} J_{\mathrm{C}-\mathrm{P}}=16.1 \mathrm{~Hz}, i-\mathrm{C}\right) .{ }^{31} \mathrm{P}\{\mathrm{H}\}$ $\operatorname{NMR}\left(\mathrm{C}_{6} \mathrm{D}_{6}, 162.0 \mathrm{MHz}\right): \delta-15.7$.

P2: ${ }^{1} \mathrm{H}$ NMR $\left(\mathrm{C}_{6} \mathrm{D}_{6}, 400.20 \mathrm{MHz}\right): \delta 1.35$ (br s, $\left.3 \mathrm{H},=\mathrm{CMeMe}(\mathrm{cis})\right), 1.53\left(\mathrm{dd},{ }^{4} \mathrm{~J}_{\mathrm{H}-\mathrm{H}}=4.1 \mathrm{~Hz},{ }^{5} J_{\mathrm{H}-\mathrm{P}}=1.4 \mathrm{~Hz}, 2 \mathrm{H}, 3 \mathrm{H}\right.$, $=\mathrm{CMeMe}(\operatorname{trans})), 2.77\left(\mathrm{~d},{ }^{4} J_{\mathrm{H}-\mathrm{P}}=7.9 \mathrm{~Hz}, 2 \mathrm{H}, \mathrm{P}-\mathrm{CH}_{2}-\right), 5.28(\mathrm{~m}, 1 \mathrm{H},-\mathrm{CH}=), 7.08(\mathrm{~m}, 6 \mathrm{H}, m-$ and $p-\mathrm{H}), 7.45(\mathrm{~m}, 4 \mathrm{H}, o-\mathrm{H})$. ${ }^{13} \mathrm{C}$ NMR $\left(\mathrm{C}_{6} \mathrm{D}_{6}, 100.6 \mathrm{MHz}\right): \delta 17.81(\mathrm{~s},=\mathrm{CMeMe}(\mathrm{cis})), 25.84\left(\mathrm{~d},{ }^{4} J_{\mathrm{C}-\mathrm{P}}=2.1 \mathrm{~Hz},=\mathrm{CMeMe}(\operatorname{trans})\right), 28.63\left(\mathrm{~d},{ }^{1} J_{\mathrm{C}-\mathrm{P}}=13.6\right.$ $\left.\mathrm{Hz}, \mathrm{P}-\mathrm{CH}_{2}-\right), 119.01\left(\mathrm{~d},{ }^{2} J_{\mathrm{C}-\mathrm{P}}=8.7 \mathrm{~Hz},-\mathrm{CH}=\right), 128.56$ (s, $\left.p-\mathrm{C}\right), 128.62(\mathrm{~s}, m-\mathrm{C}), 133.29\left(\mathrm{~d},{ }^{2} J_{\mathrm{C}-\mathrm{P}}=18.3 \mathrm{~Hz} o-\mathrm{C}\right), 134.33(\mathrm{~d}$, $\left.{ }^{3} J_{\mathrm{C}-\mathrm{P}}=17.0 \mathrm{~Hz},=\mathrm{CMe}_{2}\right) 139.65\left(\mathrm{~d},{ }^{3} J_{\mathrm{C}-\mathrm{P}}=16.7 \mathrm{~Hz}, i-\mathrm{C}\right) .{ }^{31} \mathrm{P}\{\mathrm{H}\} \mathrm{NMR}\left(\mathrm{C}_{6} \mathrm{D}_{6}, 162.0 \mathrm{MHz}\right): \delta-15.7$.

P3: ${ }^{1} \mathrm{H}$ NMR ( $\left.\mathrm{C}_{6} \mathrm{D}_{6}, 400.20 \mathrm{MHz}\right): \delta 1.79$ (s, 3H, -C(Me)=), $2.72\left(\mathrm{~s}, 2 \mathrm{H}, \mathrm{P}-\mathrm{CH}_{2}-\right), 4.66\left(\mathrm{ddq},{ }^{4} \mathrm{~J}_{\mathrm{H}-\mathrm{H}}=3.2 \mathrm{~Hz},{ }^{4} \mathrm{~J}_{\mathrm{H}-\mathrm{P}}=2.1 \mathrm{~Hz}\right.$, ${ }^{2} J_{\mathrm{H}-\mathrm{H}}=1.0 \mathrm{~Hz}, 1 \mathrm{H},-\mathrm{C}(\mathrm{Me})=\mathrm{CHH}(\mathrm{cis}), 4.72\left(\mathrm{ddq},{ }^{4} J_{\mathrm{H}-\mathrm{H}}=1.7 \mathrm{~Hz},{ }^{4} J_{\mathrm{H}-\mathrm{P}}=1.6 \mathrm{~Hz},{ }^{2} J_{\mathrm{H}-\mathrm{H}}=1.6 \mathrm{~Hz}, 1 \mathrm{H},-\mathrm{C}(\mathrm{Me})=\mathrm{CHH}(\operatorname{trans})\right.$, $7.07(\mathrm{~m}, 6 \mathrm{H}, m$ - and $p-\mathrm{H}), 7.41(\mathrm{~m}, 4 \mathrm{H}, o-\mathrm{H}) .{ }^{13} \mathrm{C}$ NMR $\left(\mathrm{C}_{6} \mathrm{D}_{6}, 100.6 \mathrm{MHz}\right): \delta 23.78\left(\mathrm{~d},{ }^{3} J_{\mathrm{C}-\mathrm{P}}=7.6 \mathrm{~Hz},-\mathrm{C}(\mathrm{Me})=\right), 39.09(\mathrm{~d}$, 
$\left.{ }^{1} J_{\mathrm{C}-\mathrm{P}}=15.5 \mathrm{~Hz}, \mathrm{P}-\mathrm{CH}_{2}-\right), 113.27\left(\mathrm{~d},{ }^{3} J_{\mathrm{C}-\mathrm{P}}=9.3 \mathrm{~Hz},=\mathrm{CH}_{2}\right), 128.60\left(\mathrm{~s},{ }^{3} J_{\mathrm{C}-\mathrm{P}}=6.6 \mathrm{~Hz}, m-\mathrm{C}\right), 128.72(p-\mathrm{C}), 133.29\left(\mathrm{~d},{ }^{2} J_{\mathrm{C}-\mathrm{P}}=\right.$ $18.8 \mathrm{~Hz}, o-\mathrm{C}), 139.68\left(\mathrm{~d},{ }^{2} J_{\mathrm{C}-\mathrm{P}}=15.5 \mathrm{~Hz},-\mathrm{C}(\mathrm{Me})=\right), 141.62\left(\mathrm{~d},{ }^{1} J_{\mathrm{C}-\mathrm{P}}=8.0 \mathrm{~Hz}, i-\mathrm{C}\right) .{ }^{31} \mathrm{P}\{\mathrm{H}\} \mathrm{NMR}\left(\mathrm{C}_{6} \mathrm{D}_{6}, 162.0 \mathrm{MHz}\right): \delta-$ 17.9.

\section{Procedure B: Synthesis of diallyl phenyl phosphine P4}

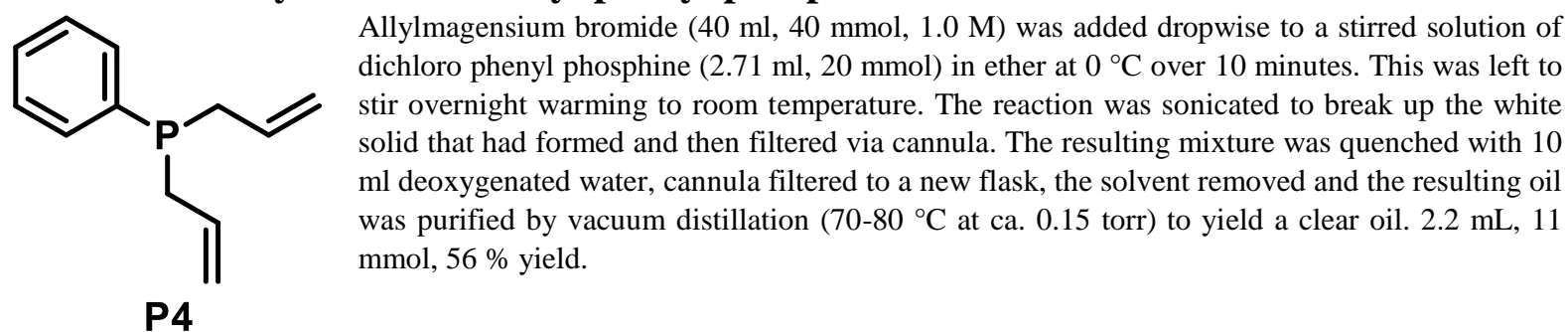

P4: ${ }^{1} \mathrm{H}$ NMR $\left(\mathrm{C}_{6} \mathrm{D}_{6}, 400.20 \mathrm{MHz}\right): \delta 2.38\left(\mathrm{~m}, 4 \mathrm{H}, \mathrm{P}-\mathrm{CH}_{2}-\right), 4.91\left(\mathrm{~m}, 4 \mathrm{H},=\mathrm{CH}_{2}\right), 5.68\left(\mathrm{ddtd},{ }^{3} J_{\mathrm{H}-\mathrm{H}(\mathrm{trans})}=16.6 \mathrm{~Hz},{ }^{3} J_{\mathrm{H}-\mathrm{H}(\mathrm{cis})}=\right.$ $\left.10.4 \mathrm{~Hz},{ }^{3} J_{\mathrm{H}-\mathrm{H} 2}=7.5 \mathrm{~Hz},{ }^{3} J_{\mathrm{H}-\mathrm{P}}=4.9 \mathrm{~Hz}, 2 \mathrm{H},-\mathrm{CH}=\right), 7.11(\mathrm{~m}, 3 \mathrm{H}, m-$ and $p-\mathrm{H}), 7.38(\mathrm{~m}, 2 \mathrm{H}, o-\mathrm{H}) .{ }^{13} \mathrm{C}$ NMR $\left(\mathrm{C}_{6} \mathrm{D}_{6}, 100.6\right.$ MHz): $\delta 32.35\left(\mathrm{~d},{ }^{1} J_{\mathrm{C}-\mathrm{P}}=16.5 \mathrm{~Hz}, \mathrm{P}-\mathrm{CH}_{2}\right), 116.84\left(\mathrm{~d},{ }^{3} J_{\mathrm{C}-\mathrm{P}}=8.8 \mathrm{~Hz},=\mathrm{CH}_{2}\right), 128.49\left(\mathrm{~d},{ }^{3} J_{\mathrm{C}-\mathrm{P}}=8.8 \mathrm{~Hz}, m-\mathrm{C}\right), 128.83(\mathrm{~s}, p-$ C), $132.66\left(\mathrm{~d},{ }^{1} J_{\mathrm{C}-\mathrm{P}}=18.5 \mathrm{~Hz}, o-\mathrm{C}\right), 133.56\left(\mathrm{~d},{ }^{1} J_{\mathrm{C}-\mathrm{P}}=7.3 \mathrm{~Hz},-\mathrm{CH}=\right), 138.04\left(\mathrm{~d},{ }^{1} J_{\mathrm{C}-\mathrm{P}}=19.6 \mathrm{~Hz}, i-\mathrm{C}\right) .{ }^{31} \mathrm{P}\{\mathrm{H}\} \mathrm{NMR}\left(\mathrm{C}_{6} \mathrm{D}_{6}\right.$, $162.0 \mathrm{MHz}): \delta-26.6$.

\section{Procedure C: Synthesis of analogous phosphine oxides P1', P2', P3' and P4'}<smiles>C=CCP(=O)(c1ccccc1)c1ccccc1</smiles>

P1'<smiles>CC(C)=CCP(=O)(c1ccccc1)c1ccccc1</smiles>

P2'<smiles>C=C(C)CP(=O)(c1ccccc1)c1ccccc1</smiles>

P3'<smiles>C=CCP(=O)(CC=C)c1ccccc1</smiles>

P4'

The parent phosphine (P1, P2, P3 or P4) was dissolved in THF, cooled to $0{ }^{\circ} \mathrm{C}$ and an excess of dilute $\mathrm{H}_{2} \mathrm{O}_{2}$ in THF was added to it dropwise. An organic extraction using $\mathrm{Et}_{2} \mathrm{O}$ was carried out followed by drying with $\mathrm{MgSO}_{4}$. Removal of the solvent under vacuum yielded phosphine oxides P1'-3' as crystalline white solids and P4' as a thick colourless oil (65, 50, 57 and 4 $\%$ yield respectively).

P1': ${ }^{1} \mathrm{H}$ NMR (C6 6 6, 400.20 MHz): $\delta 2.76\left(\mathrm{ddt},{ }^{2} J_{\mathrm{H}-\mathrm{P}}=14.4 \mathrm{~Hz},{ }^{3} J_{\mathrm{H}-\mathrm{H}}=7.4 \mathrm{~Hz},{ }^{4} J_{\mathrm{H}-\mathrm{H}}=1.3 \mathrm{~Hz}, 2 \mathrm{H}, \mathrm{P}-\mathrm{CH}_{2}-\right), 4.95(\mathrm{~m}, 2 \mathrm{H}$, $\left.=\mathrm{CH}_{2}\right), 5.84(\mathrm{~m}, 1 \mathrm{H},-\mathrm{CH}=), 7.04(\mathrm{~m}, 6 \mathrm{H}, m-$ and $p-\mathrm{H}), 7.71(\mathrm{~m}, 4 \mathrm{H}, o-\mathrm{H}) .{ }^{13} \mathrm{C} \mathrm{NMR}\left(\mathrm{C}_{6} \mathrm{D}_{6}, 100.6 \mathrm{MHz}\right): \delta 34.07\left(\mathrm{~d},{ }^{1} J_{\mathrm{C}-\mathrm{P}}=\right.$ $\left.14.9 \mathrm{~Hz}, \mathrm{P}-\mathrm{CH}_{2}-\right), 117.35\left(\mathrm{~d},{ }^{3} J_{\mathrm{C}-\mathrm{P}}=10.7 \mathrm{~Hz},=\mathrm{CH}_{2}\right), 128.67(\mathrm{~m}, m$ and $p-\mathrm{C}), 133.26\left(\mathrm{~d},{ }^{1} J_{\mathrm{C}-\mathrm{P}}=18.6 \mathrm{~Hz}, o-\mathrm{C}\right), 133.61\left(\mathrm{~d},{ }^{1} J_{\mathrm{C}-}\right.$ $\mathrm{P}=9.3 \mathrm{~Hz}, i-\mathrm{C}), 139.09\left(\mathrm{~d},{ }^{1} J_{\mathrm{C}-\mathrm{P}}=16.1 \mathrm{~Hz},-\mathrm{CH}=\right) .{ }^{31} \mathrm{P}\{\mathrm{H}\} \mathrm{NMR}\left(\mathrm{C}_{6} \mathrm{D}_{6}, 162.0 \mathrm{MHz}\right): \delta 25.9$.

P2': ${ }^{1} \mathrm{H}$ NMR ( $\left.\mathrm{C}_{6} \mathrm{D}_{6}, 400.20 \mathrm{MHz}\right): \delta 1.37\left(\mathrm{dd},{ }^{4} J_{\mathrm{H}-\mathrm{H}}=3.4 \mathrm{~Hz},{ }^{5} J_{\mathrm{H}-\mathrm{P}}=1.3 \mathrm{~Hz}, 3 \mathrm{H},=\mathrm{CMe}_{2}(\mathrm{cis})\right), 1.48\left(\mathrm{dd},{ }^{4} J_{\mathrm{H}-\mathrm{H}}=4.5 \mathrm{~Hz},{ }^{5} J_{\mathrm{H}-}\right.$ $\left.\mathrm{P}=1.3 \mathrm{~Hz}, 3 \mathrm{H},=\mathrm{CMe}_{2}(\operatorname{trans})\right), 2.86\left(\mathrm{dd},{ }^{3} J_{\mathrm{H}-\mathrm{H}}=14.4 \mathrm{~Hz},{ }^{4} J_{\mathrm{H}-\mathrm{P}}=7.7 \mathrm{~Hz}, 2 \mathrm{H}, \mathrm{P}_{-} \mathrm{CH}_{2}-\right), 5.33(\mathrm{~m}, 1 \mathrm{H},-\mathrm{CH}=), 7.05(\mathrm{~m}, 6 \mathrm{H}, m-$ and $p-\mathrm{H}), 7.77(\mathrm{~m}, 4 \mathrm{H}, o-\mathrm{H}) .{ }^{13} \mathrm{C} \mathrm{NMR}\left(\mathrm{C}_{6} \mathrm{D}_{6}, 100.6 \mathrm{MHz}\right): \delta 18.13\left(\mathrm{~d},{ }^{4} J_{\mathrm{C}-\mathrm{P}}=2.5 \mathrm{~Hz},=\mathrm{CMe}_{2}(\mathrm{cis})\right), 25.81\left(\mathrm{~d},{ }^{4} J_{\mathrm{C}-\mathrm{P}}=2.8 \mathrm{~Hz}\right.$, $=\mathrm{CMe}_{2}$ (trans)), $31.37\left(\mathrm{~d},{ }^{1} J_{\mathrm{C}-\mathrm{P}}=70.0 \mathrm{~Hz}, \mathrm{P}-\mathrm{CH}_{2}-\right), 113.44\left(\mathrm{~d},{ }^{3} J_{\mathrm{C}-\mathrm{P}}=9.2 \mathrm{~Hz},-\mathrm{CH}=\right), 128.55\left(\mathrm{~d},{ }^{3} J_{\mathrm{C}-\mathrm{P}}=9.2 \mathrm{~Hz}, m-\mathrm{C}\right), 131.3$ $(\mathrm{s}, p-\mathrm{C}), 131.35\left(\mathrm{~d},{ }^{2} J_{\mathrm{C}-\mathrm{P}}=9.04 \mathrm{~Hz}, o-\mathrm{C}\right), 135.02\left(\mathrm{~d},{ }^{1} J_{\mathrm{C}-\mathrm{P}}=96.0 \mathrm{~Hz}, i-\mathrm{C}\right), 137.02\left(\mathrm{~d},{ }^{4} J_{\mathrm{C}-\mathrm{P}}=11.9 \mathrm{~Hz},=\mathrm{CMe} 2\right) .{ }^{31} \mathrm{P}\{\mathrm{H}\} \mathrm{NMR}$ $\left(\mathrm{C}_{6} \mathrm{D}_{6}, 162.0 \mathrm{MHz}\right): \delta 26.7$.

P3': ${ }^{1} \mathrm{H}$ NMR $\left(\mathrm{C}_{6} \mathrm{D}_{6}, 400.20 \mathrm{MHz}\right): \delta 1.91(\mathrm{~m}, 3 \mathrm{H},-\mathrm{C}(\mathrm{Me})=), 2.80\left(\mathrm{~d},{ }^{3} J_{\mathrm{H}-\mathrm{H}}=13.9 \mathrm{~Hz}, 2 \mathrm{H}, \mathrm{P}-\mathrm{CH}_{2}-\right), 4.63\left(\mathrm{ddq},{ }^{2} J_{\mathrm{H}-\mathrm{H}}=4.7\right.$ $\left.\mathrm{Hz},{ }^{4} J_{\mathrm{H}-\mathrm{P}}=1.9 \mathrm{~Hz},{ }^{4} J_{\mathrm{H}-\mathrm{H}}=0.9 \mathrm{~Hz}, 1 \mathrm{H},=\mathrm{CHH}(\mathrm{cis})\right), 4.77\left(\mathrm{ddq},{ }^{2} J_{\mathrm{H}-\mathrm{H}}=4.7 \mathrm{~Hz},{ }^{4} J_{\mathrm{H}-\mathrm{P}}=1.6 \mathrm{~Hz},{ }^{4} J_{\mathrm{H}-\mathrm{H}}=1.6 \mathrm{~Hz}, 1 \mathrm{H}\right.$, $=\mathrm{CHH}(\operatorname{trans})), 7.05(\mathrm{~m}, 6 \mathrm{H}, m$ - and $p-\mathrm{H}), 7.76\left(\mathrm{~m}, 4 \mathrm{H}, o-\mathrm{H} .{ }^{13} \mathrm{C}\right.$ NMR $\left(\mathrm{C}_{6} \mathrm{D}_{6}, 100.6 \mathrm{MHz}\right): \delta 24.76\left(\mathrm{~d},{ }^{3} J_{\mathrm{C}-\mathrm{P}}=2.2 \mathrm{~Hz},-\right.$ $\mathrm{C}(\mathrm{Me})=), 38.89\left(\mathrm{~d},{ }^{3} J_{\mathrm{C}-\mathrm{P}}=66.7 \mathrm{~Hz}, \mathrm{P}-\mathrm{CH}_{2}-\right), 115.60\left(\mathrm{~d},{ }^{3} J_{\mathrm{C}-\mathrm{P}}=9.8 \mathrm{~Hz},=\mathrm{CH}_{2}\right), 128.54\left(\mathrm{~d},{ }^{3} J_{\mathrm{C}-\mathrm{P}}=11.5 \mathrm{~Hz}, m-\mathrm{C}\right), 131.34(\mathrm{~d}$, $\left.{ }^{4} J_{\mathrm{C}-\mathrm{P}}=2.8 \mathrm{~Hz}, p-\mathrm{C}\right), 131.34\left(\mathrm{~d},{ }^{3} J_{\mathrm{C}-\mathrm{P}}=8.8 \mathrm{~Hz}, o-\mathrm{C}\right), 134.70\left(\mathrm{~d},{ }^{3} J_{\mathrm{C}-\mathrm{P}}=96.9 \mathrm{~Hz},-\mathrm{C}(\mathrm{Me})=\right), 137.43\left(\mathrm{~d},{ }^{3} J_{\mathrm{C}-\mathrm{P}}=9.6 \mathrm{~Hz}, i-\mathrm{C}\right)$. ${ }^{31} \mathrm{P}\{\mathrm{H}\}$ NMR $\left(\mathrm{C}_{6} \mathrm{D}_{6}, 162.0 \mathrm{MHz}\right): \delta 25.7$.

P4': ${ }^{1} \mathrm{H}$ NMR ( $\left.\mathrm{C}_{6} \mathrm{D}_{6}, 400.20 \mathrm{MHz}\right): \delta 2.45\left(\mathrm{~m}, 4 \mathrm{H}, \mathrm{P}-\mathrm{CH}_{2}-\right), 4.91\left(\mathrm{~m}, 4 \mathrm{H},=\mathrm{CH}_{2}\right), 5.73\left(\mathrm{ddtd},{ }^{3} J_{\mathrm{H}-\mathrm{P}}=17.3 \mathrm{~Hz},{ }^{3} J_{\mathrm{H}-\mathrm{H}(\text { trans })}=\right.$ $\left.10.2 \mathrm{~Hz},{ }^{3} J_{\mathrm{H}-\mathrm{H}}=7.5 \mathrm{~Hz},{ }^{3} J_{\mathrm{H}-\mathrm{H}(\mathrm{cis})}=5.4 \mathrm{~Hz}, 2 \mathrm{H},-\mathrm{CH}=\right), 7.08(\mathrm{~m}, 3 \mathrm{H}, m-$ and $p-\mathrm{H}), 7.59(\mathrm{~m}, 2 \mathrm{H}, o-\mathrm{H}),{ }^{13} \mathrm{C}$ NMR $\left(\mathrm{C}_{6} \mathrm{D}_{6}, 100.6\right.$ $\mathrm{MHz}): \delta 35.79\left(\mathrm{~d},{ }^{1} J_{\mathrm{C}-\mathrm{P}}=65.2 \mathrm{~Hz}, \mathrm{P}-\mathrm{CH}_{2}-\right), 119.99\left(\mathrm{~d},{ }^{3} J_{\mathrm{C}-\mathrm{P}}=11.4 \mathrm{~Hz},=\mathrm{CH}_{2}\right), 128.40\left(\mathrm{~d},{ }^{2} J_{\mathrm{C}-\mathrm{P}}=9.0 \mathrm{~Hz},-\mathrm{CH}=\right), 128.48(\mathrm{~d}$, $\left.{ }^{2} J_{\mathrm{C}-\mathrm{P}}=11.2 \mathrm{~Hz}, m-\mathrm{C}\right), 131.21\left(\mathrm{~d},{ }^{1} J_{\mathrm{C}-\mathrm{P}}=8.5 \mathrm{~Hz}, o-\mathrm{C}\right), 131.47\left(\mathrm{~d},{ }^{1} J_{\mathrm{C}-\mathrm{P}}=2.8 \mathrm{~Hz}, p-\mathrm{C}\right), 133.25\left(\mathrm{~d},{ }^{1} J_{\mathrm{C}-\mathrm{P}}=92.9 \mathrm{~Hz}, i-\mathrm{C}\right)$.

${ }^{31} \mathrm{P}\{\mathrm{H}\}$ NMR $\left(\mathrm{C}_{6} \mathrm{D}_{6}, 162.0 \mathrm{MHz}\right): \delta 31.8$. 


\section{Crystallographic Data}

Crystals of P'1-4, 1, 2, 3a, 3b, 6 and 8-12, suitable for X-ray structural determination were mounted in silicone oil. Crystallographic measurements were carried out with either a Bruker X8 APEXII CCD diffractometer with a graphite monochromator with Mo K $\alpha$ radiation $(\lambda=0.71073 \AA)$; or a Rigaku Xtalab Synergy Dualflex using a graphite monochromator with Mo K $\alpha$ radiation $(\lambda=0.71073 \AA)$ or $\mathrm{Cu} \mathrm{K \alpha}$ radiation $(\lambda=1.54180 \AA)$; or the MX1 beamline of the Australian Synchrotron $(\lambda=0.71090 \AA) .{ }^{1}$ Structures were solved and refined with SHELX-2016 ${ }^{2}$ suite and X-seed interface ${ }^{3}$ or Olex $2 .{ }^{4}$ Hydrogen atoms were typically included in calculated positions (riding model). Data for P'1-4, 1, 2, 3a, 3b, 6 and 8-12 has been deposited with the Cambridge Crystallographic Database with CCDC numbers 1953196, 1953195, 1953200, 1953198, 1953207, 1953206, 1953202, 1953199, 1953205, 1953204, 1953197, 1953203, 1953194 and 1953201 respectively. Crystal data, details of data collections and refinements for all structures can be found in their CIF files and are summarized in the text below. The supplementary crystallographic data for this paper can be obtained free of charge from the Cambridge Crystallographic Data Centre (CCDC) via www.ccdc.cam.ac.uk/data_request/cif. 


\section{Crystallographic data for P1':}

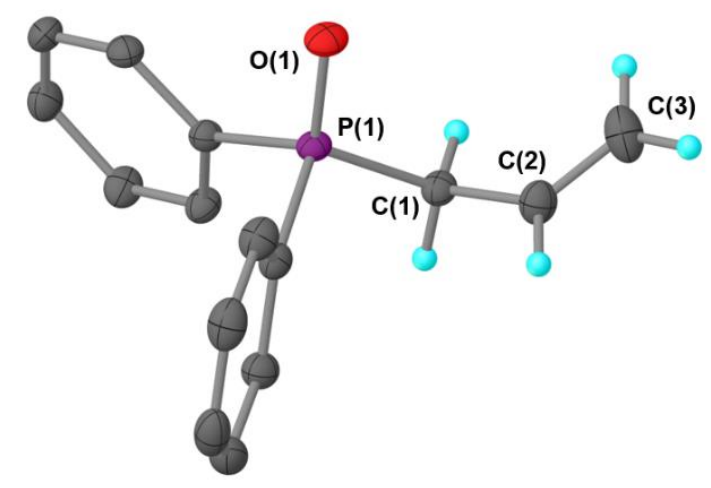

Figure S1. Structure of allyldiphenylphosphine oxide $\mathrm{Ph}_{2} \mathrm{P}(\mathrm{O}) \mathrm{CH}_{2} \mathrm{CHCH}_{2}$ P1'. Hydrogen atoms (except allylic) were omitted for clarity. Thermal ellipsoids are shown at a $40 \%$ probability. Selected bond lengths $(\AA)$ and angles $\left({ }^{\circ}\right)$ : $\mathrm{P}(1)-\mathrm{C}(1), 1.8093(13)$; $\mathrm{C}(1)$ $\mathrm{C}(2), 1.4958(18) ; \mathrm{C}(2)-\mathrm{C}(3), 1.310(2) ; \mathrm{P}(1)-\mathrm{O}(1), 1.4901 ; \mathrm{P}(1)-\mathrm{C}(1)-\mathrm{C}(2), 112.37(9)$.

Crystal data for allyldiphenylphosphine oxide P1', $\mathrm{C}_{15} \mathrm{H}_{15} \mathrm{OP}, 1953196$ (CCDC): $M=242.26$, clear whiteish colourless needle, $0.545 \times 0.323 \times 0.27 \mathrm{~mm}^{3}$, space group (No. 14), $V=1297.54(4) \AA^{3}, Z=4, D_{\mathrm{c}}=1.240 \mathrm{~g} / \mathrm{cm}^{3}, F_{000}=512$, XtaLAB Synergy, Dualflex, HyPix, CuK $\alpha$ radiation, $\lambda=1.54184 \AA$, $T=123.00(10) \mathrm{K}, 2 \theta_{\max }=153.6^{\circ}, 9175$ reflections collected, 2665 unique $\left(R_{\text {int }}=0.0284\right)$. The structure was solved and refined using the programs SHELXT 2014/5 (Sheldrick, 2014) and ShelXL (Sheldrick, 2016) respectively. The program Olex $2^{4}$ was used as an interface to the SHELX programs, and to prepare the figures. Final $G o o F=1.071, R 1=0.0364, w R 2=0.0987, R$ indices based on 2587 reflections with $\mathrm{I}>2 \sigma(\mathrm{I})$ (refinement on $F^{2}$ ), 162 parameters, 0 restraints. Lp and absorption corrections applied, $\mu=1.709 \mathrm{~mm}^{-1}$. 


\section{Crystallographic data for P2':}

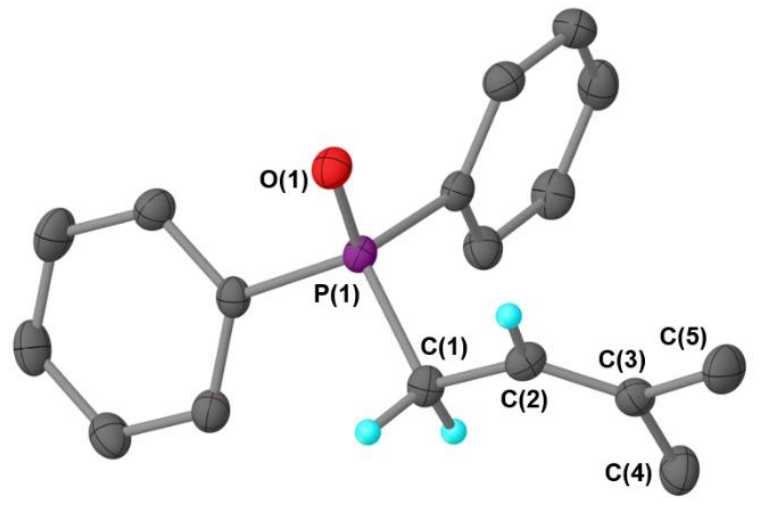

Figure S2. Structure of 3,3-dimethylallyldiphenylphosphine oxide $\mathrm{Ph}_{2} \mathrm{P}(\mathrm{O}) \mathrm{CH}_{2} \mathrm{CHCMe}_{2} \mathbf{P 2}$ '. Hydrogen atoms (except allylic and including allyl- $\left.\mathrm{Me}_{2}\right)$ were omitted for clarity. Thermal ellipsoids are shown at a $40 \%$ probability. Selected bond lengths $(\AA)$ and angles $\left({ }^{\circ}\right)$ : $\mathrm{P}(1)-\mathrm{C}(1), 1.8034(17) ; \mathrm{C}(1)-\mathrm{C}(2), 1.505(2) ; \mathrm{C}(2)-\mathrm{C}(3), 1.331(2) ; \mathrm{P}(1)-\mathrm{O}(1), 1.4912(13) ; \mathrm{P}(1)-\mathrm{C}(1)-\mathrm{C}(2), 111.35(12)$.

Crystal data for 3,3-dimethylallyldiphenylphosphine oxide P2', $\mathrm{C}_{17} \mathrm{H}_{19} \mathrm{OP}, 1953195$ (CCDC): $M=270.31$, clear whiteish colourless needle, $0.494 \times 0.139 \times 0.043 \mathrm{~mm}^{3}$, space group (No. 14), $V=1449.92(4) \AA^{3}, Z=4, D_{\mathrm{c}}=1.238 \mathrm{~g} / \mathrm{cm}^{3}, F_{000}=576$, XtaLAB Synergy, Dualflex, HyPix, CuK $\alpha$ radiation, $\lambda=1.54184 \AA ̊ 丿, T=123.00(10) \mathrm{K}, 2 \theta_{\max }=154.0^{\circ}, 10960$ reflections collected, 2980 unique $\left(\mathrm{R}_{\text {int }}=0.0426\right)$. The structure was solved and refined using the programs SHELXT 2018/2 (Sheldrick, 2018) and ShelXL (Sheldrick, 2016) respectively. The program Olex $2^{4}$ was used as an interface to the SHELX programs, and to prepare the figures. Final $G o o F=1.071, R l=0.0440, w R 2=0.1092, R$ indices based on 2732 reflections with $\mathrm{I}>2 \sigma(\mathrm{I})$ (refinement on $F^{2}$ ), 174 parameters, 0 restraints. Lp and absorption corrections applied, $\mu=1.579 \mathrm{~mm}^{-1}$. 


\section{Crystallographic data for P3':}

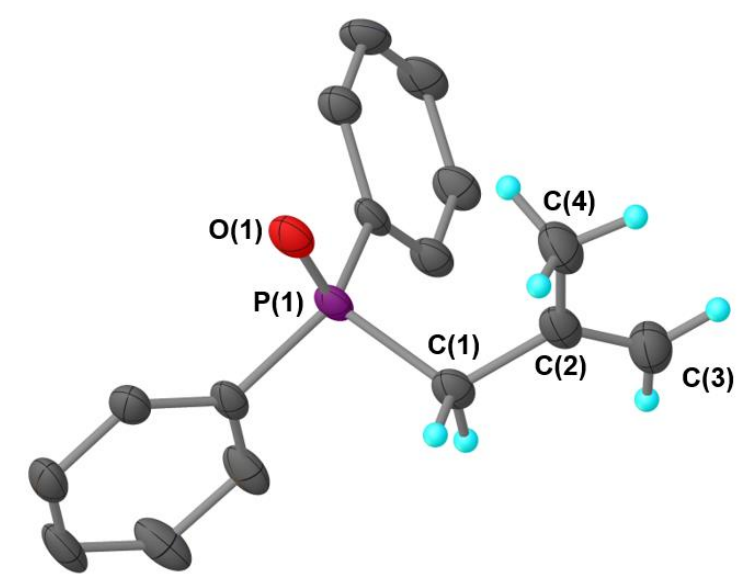

Figure S3. Structure of 2-methylallyldiphenylphosphine oxide $\mathrm{Ph}_{2} \mathrm{P}(\mathrm{O}) \mathrm{CH}_{2} \mathrm{C}(\mathrm{Me}) \mathrm{CH}_{2} \mathbf{P 3}$ '. Hydrogen atoms (except allylic) were omitted for clarity. Thermal ellipsoids are shown at a $40 \%$ probability. Selected bond lengths $(\AA)$ and angles $\left({ }^{\circ}\right)$ : $\mathrm{P}(1)-\mathrm{C}(1), 1.816(3)$; $\mathrm{C}(1)-\mathrm{C}(2), 1.513(4) ; \mathrm{C}(2)-\mathrm{C}(3), 1.338(5) ; \mathrm{C}(2)-\mathrm{C}(4), 1.474(5) ; \mathrm{P}(1)-\mathrm{O}(1), 1.475(3) ; \mathrm{P}(1)-\mathrm{C}(1)-\mathrm{C}(2), 115.7(2)$.

Crystal data for 2-methylallyldiphenylphosphine oxide P3', $\mathrm{C}_{16} \mathrm{H}_{17} \mathrm{OP}, 1953200$ (CCDC): $M=256.28$, clear whiteish colourless needle, $0.22 \times 0.142 \times 0.09 \mathrm{~mm}^{3}$, space group (No. 61), $V=2763.54(10) \AA^{3}, Z=8, D_{\mathrm{c}}=1.232 \mathrm{~g} / \mathrm{cm}^{3}, F_{000}=1088$, XtaLAB Synergy, Dualflex, HyPix, CuK $\alpha$ radiation, $\lambda=1.54178 \AA$, $T=123.00(10) \mathrm{K}, 2 \theta_{\max }=154.2^{\circ}, 14848$ reflections collected, 2865 unique $\left(\mathrm{R}_{\text {int }}=0.0812\right)$. The structure was solved and refined using the programs SHELXT 2014/5 (Sheldrick, 2014) and ShelXL (Sheldrick, 2016) respectively. The program Olex $2^{4}$ was used as an interface to the SHELX programs, and to prepare the figures. Final $G o o F=1.086, R 1=0.0704, w R 2=0.1710, R$ indices based on 2544 reflections with $\mathrm{I}>2 \sigma(\mathrm{I})$ (refinement on $F^{2}$ ), 164 parameters, 0 restraints. Lp and absorption corrections applied, $\mu=1.631 \mathrm{~mm}^{-1}$. 


\section{Crystallographic data for P4':}

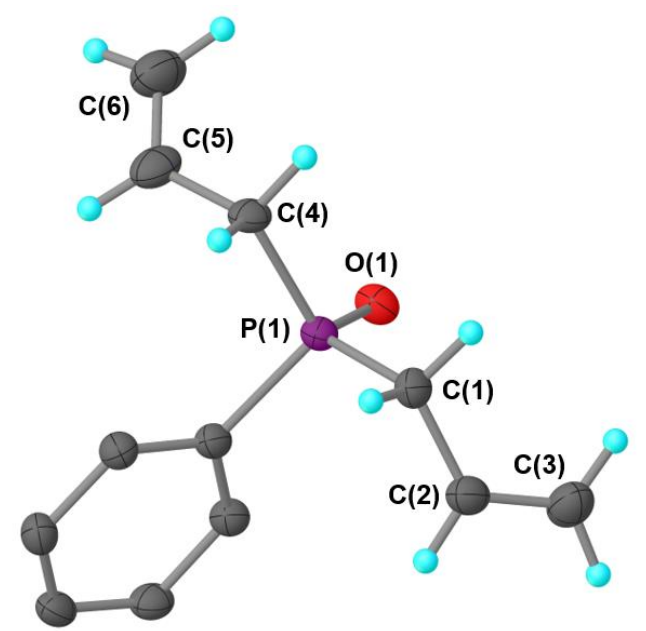

Figure S4. Structure of diallylphenylphosphine oxide $\mathrm{PhP}(\mathrm{O})\left\{\mathrm{CH}_{2} \mathrm{CHCH}_{2}\right\}_{2} \mathbf{P 4}$ '. Hydrogen atoms (except allylic) were omitted for clarity. Thermal ellipsoids are shown at a $40 \%$ probability. Selected bond lengths $(\AA)$ and angles $\left({ }^{\circ}\right)$ : $\mathrm{P}(1)-\mathrm{C}(1), 1.814(4) ; \mathrm{P}(1)-\mathrm{C}(4)$, 1.820(4); $\mathrm{C}(1)-\mathrm{C}(2), 1.513(6) ; \mathrm{C}(4)-\mathrm{C}(5), 1.498(6) ; \mathrm{C}(2)-\mathrm{C}(3), 1.289(8) ; \mathrm{C}(5)-\mathrm{C}(6), 1.308(7) ; \mathrm{P}(1)-\mathrm{O}(1), 1.495(4) ; \mathrm{P}(1)-\mathrm{C}(1)-\mathrm{C}(2)$, 112.9(3); $\mathrm{P}(1)-\mathrm{C}(4)-\mathrm{C}(5), 111.6(3)$.

Crystal data for diallylphenylphosphine oxide P4', $\mathrm{C}_{12} \mathrm{H}_{15} \mathrm{OP}, 1953198$ (CCDC): $M=206.22$, clear whiteish colourless needle, $0.452 \times 0.062 \times 0.055 \mathrm{~mm}^{3}$, space group (No. 4), $V=565.70(2) \AA^{3}, Z=2, D_{\mathrm{c}}=1.211 \mathrm{~g} / \mathrm{cm}^{3}, F_{000}=220$, XtaLAB Synergy, Dualflex, HyPix, CuK $\alpha$ radiation, $\lambda=1.54184 \AA$, $T=123.00(10) \mathrm{K}, 2 \theta_{\max }=154.0^{\circ}, 7867$ reflections collected, 2254 unique $\left(R_{\text {int }}=0.0517\right)$. The structure was solved and refined using the programs SHELXT 2014/5 (Sheldrick, 2014) and ShelXL (Sheldrick, 2016) respectively. The program Olex $2^{4}$ was used as an interface to the SHELX programs, and to prepare the figures. Final $G o o F=1.087, R I=0.0693, w R 2=0.1810, R$ indices based on 2190 reflections with $\mathrm{I}>2 \sigma(\mathrm{I})$ (refinement on $\left.F^{2}\right), 127$ parameters, 1 restraint. Lp and absorption corrections applied, $\mu=1.864 \mathrm{~mm}^{-1}$. Absolute structure parameter $=0.08(5)$ (Flack, H. D. Acta Cryst. 1983, A39, 876-881). 


\section{Crystallographic data for 1:}

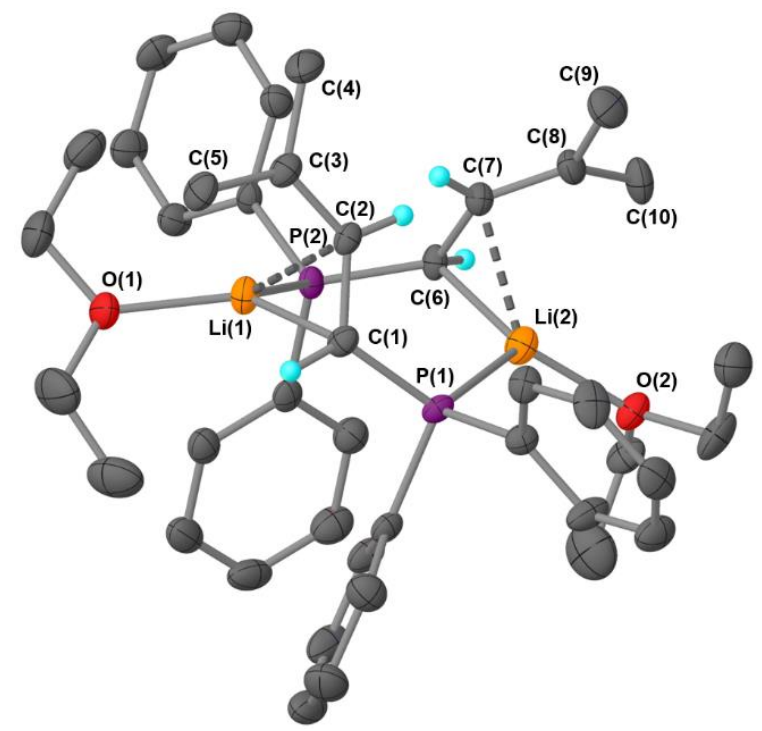

Figure S5. Molecular structure of $\left[\left\{\mathrm{Ph}_{2} \mathrm{PCHCHCMe}_{2} \mathrm{Li}_{2}\left(\mathrm{Et}_{2} \mathrm{O}\right)\right\}_{2}\right]$ 1. Hydrogen atoms (except allylic and including allyl-Me $)$ were omitted for clarity. Disorder in one of the diethyl ether molecules was omitted for clarity. Thermal ellipsoids are shown at a $40 \%$ probability. Selected bond lengths $(\AA)$ and angles ( $\left.{ }^{\circ}\right)$ : P(1)-C(1), 1.752(3); C(1)-C(2), 1.434(4); C(2)-C(3), 1.357(4); Li(1)-C(1), 2.224(6); $\mathrm{Li}(1)-\mathrm{C}(2), 2.250(6) ; \mathrm{Li}(1)-\mathrm{C}(3), 2.672(6) ; \mathrm{Li}(2)-\mathrm{P}(1), 2.612(6) ; \mathrm{Li}(2)-\mathrm{C}(6), 2.192(6) ; \mathrm{Li}(2)-\mathrm{C}(7), 2.173(6) ; \mathrm{P}(1)-\mathrm{C}(1)-\mathrm{C}(2)$, 118.4(2).

Crystal data for $\left[\left\{\mathrm{Ph}_{2} \mathrm{PCHCHCMe} 2 \mathrm{Li}\left(\mathrm{Et}_{2} \mathrm{O}\right)\right\}_{2}\right] \quad 1, \mathrm{C}_{42} \mathrm{H}_{56} \mathrm{Li}_{2} \mathrm{O}_{2} \mathrm{P}_{2}, 1953207$ (CCDC): $M=668.68$, clear orangish yellow needle, $0.16 \times 0.12 \times 0.10 \mathrm{~mm}^{3}$, space group (No. 14), $V=3938.0(4) \AA^{3}, Z=4, D_{\mathrm{c}}=1.128 \mathrm{~g} / \mathrm{cm}^{3}, F_{000}=1440$, Bruker APEXII CCD, MoK $\alpha$ radiation, $\lambda=0.71073 \AA, T=123 \mathrm{~K}, 2 \theta_{\max }=59.4^{\circ}, 65915$ reflections collected, 11174 unique $\left(\mathrm{R}_{\text {int }}=0.0456\right)$. The structure was solved and refined using the programs SHELXT 2014/5 (Sheldrick, 2014) and ShelXL (Sheldrick, 2016) respectively. The program Olex $2^{4}$ was used as an interface to the SHELX programs, and to prepare the figures. One of the diethyl ether molecules was modelled as disordered. Refined as a two-component twin (Twin law 1, 0, 0.02, 0, -1, 0, 0, 0, -1), BASF [0.0969(11)]. Final $G o o F=1.086, R 1=0.0759, w R 2=0.1816, R$ indices based on 9321 reflections with $\mathrm{I}>2 \sigma(\mathrm{I})$ (refinement on $F^{2}$ ), 484 parameters, 6 restraints. Lp and absorption corrections applied, $\mu=0.143 \mathrm{~mm}^{-1}$. 


\section{Crystallographic data for 2:}

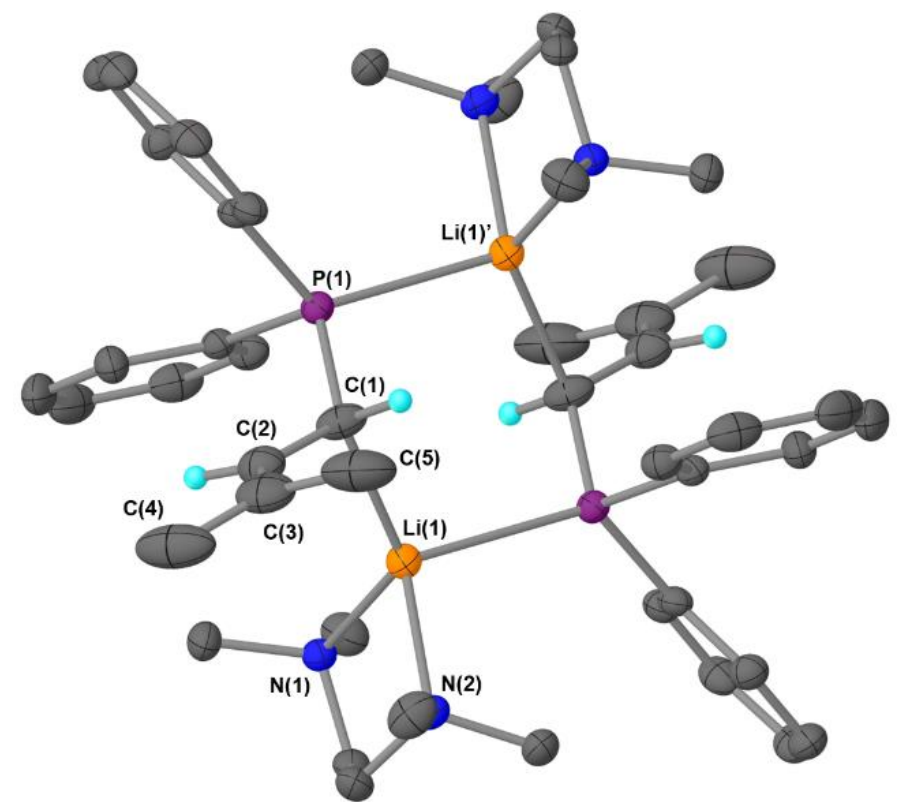

Figure S6. Molecular structure of [ $\left.\left\{\mathrm{Ph}_{2} \mathrm{PCHCHCMe} \mathrm{Li}_{2}(\mathrm{TMEDA})\right\}_{2}\right]$ 2. Hydrogen atoms (except allylic and including allyl-Me $\mathrm{Me}_{2}$ ) were omitted for clarity. Thermal ellipsoids are shown at a $40 \%$ probability. Symmetry operator (') $=1-\mathrm{x}, 1-\mathrm{y}, 1-\mathrm{z}$. Selected bond lengths $(\AA)$ and angles $\left({ }^{\circ}\right)$ : $\mathrm{P}(1)-\mathrm{C}(1), 1.753(2) ; \mathrm{C}(1)-\mathrm{C}(2), 1.419(3)$; $\mathrm{C}(2)-\mathrm{C}(3), 1.354(4) ; \mathrm{Li}(1)-\mathrm{C}(1), 2.216(4)$; $\mathrm{Li}(1)$ ') $-\mathrm{P}(1), 2.655(4)$; $\mathrm{P}(1)-\mathrm{C}(1)-\mathrm{C}(2), 121.44(18)$.

Crystal data for $\left[\left\{\mathrm{Ph}_{2} \mathrm{PCHCHCMe} 2 \mathrm{Li}(\mathrm{TMEDA})\right\}_{2}\right] 2, \mathrm{C}_{46} \mathrm{H}_{68} \mathrm{Li}_{2} \mathrm{~N}_{4} \mathrm{P}_{2}, 1953206$ (CCDC): $M=752.86$, clear orangish yellow block, $0.312 \times 0.254 \times 0.121 \mathrm{~mm}^{3}$, space group (No. 2), $V=1096.72(18) \AA^{3}, Z=1, D_{\mathrm{c}}=1.140 \mathrm{~g} / \mathrm{cm}^{3}, F_{000}=408$, Bruker APEX-II CCD, MoK $\alpha$ radiation, $\lambda=0.71073 \AA, T=123 \mathrm{~K}, 2 \theta_{\max }=61.6^{\circ}, 36072$ reflections collected, 6792 unique $\left(\mathrm{R}_{\text {int }}=\right.$ 0.0445). The structure was solved and refined using the programs SHELXT 2014/5 (Sheldrick, 2014) and ShelXL (Sheldrick, 2016) respectively. The program Olex $2^{4}$ was used as an interface to the SHELX programs, and to prepare the figures. Final $G o o F=1.042, R 1=0.0662, w R 2=0.1638, R$ indices based on 4960 reflections with $\mathrm{I}>2 \sigma(\mathrm{I})\left(\right.$ refinement on $\left.F^{2}\right), 250$ parameters, 0 restraints. Lp and absorption corrections applied, $\mu=0.134 \mathrm{~mm}^{-1}$. 


\section{Crystallographic data for 3:}
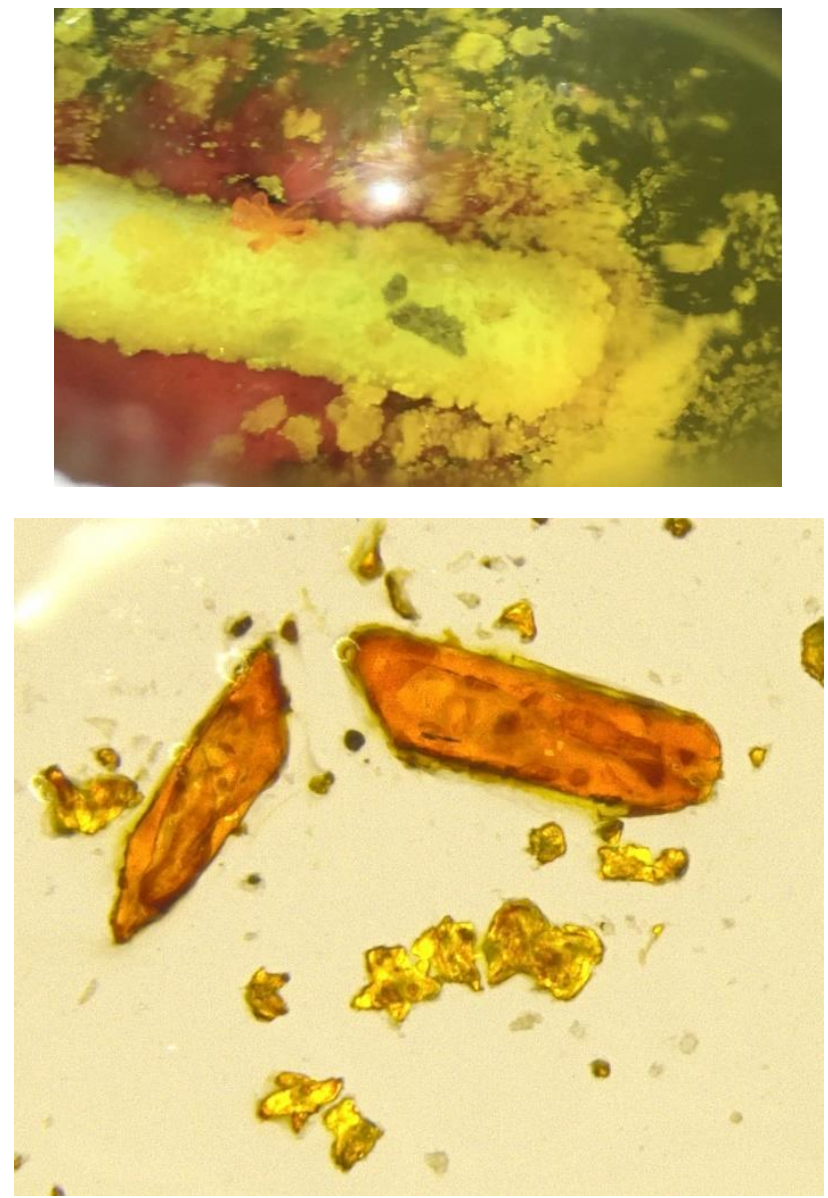

Figure S7. Top - polymorphs 3a (orange) and $\mathbf{3 b}$ (yellow) in the reaction mixture. Bottom - larger orange crystals of $\mathbf{3 a}$ and smaller yellow crystals of $\mathbf{3 b}$ under a microscope.

3a:

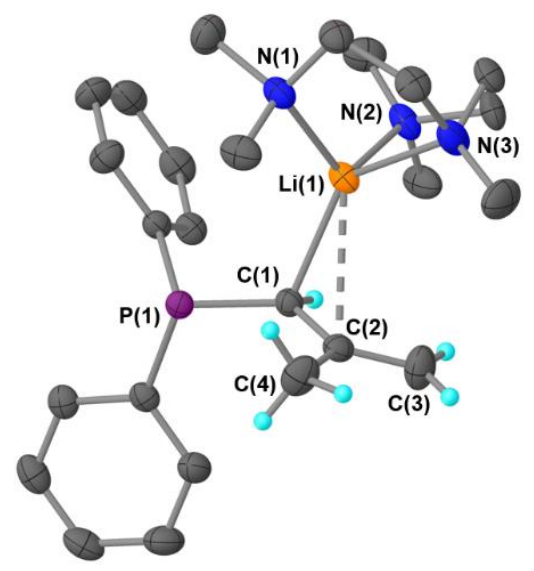

Figure S8. Molecular structures of $\left[\mathrm{Ph}_{2} \mathrm{PCHC}(\mathrm{Me}) \mathrm{CH}_{2} \mathrm{Li}(\mathrm{PMDETA})\right]$ 3a. Hydrogen atoms (except allylic) were omitted for clarity. Disorder in the PMDETA molecule was omitted for clarity. Thermal ellipsoids are shown at a $40 \%$ probability. Selected bond lengths $(\AA)$ and angles $\left({ }^{\circ}\right)$ : $\mathrm{P}(1)-\mathrm{C}(1), 1.7636(12) ; \mathrm{C}(1)-\mathrm{C}(2), 1.4447(17) ; \mathrm{C}(2)-\mathrm{C}(3), 1.367(2) ; \mathrm{Li}(1)-\mathrm{C}(1), 2.282(2) ; \mathrm{Li}(1)-\mathrm{C}(2), 2.760(3)$; $\mathrm{Li}(1)-\mathrm{C}(3), 3.290(3) ; \mathrm{C}(2)-\mathrm{C}(4), 1.4866(19) ; \mathrm{P}(1)-\mathrm{C}(1)-\mathrm{C}(2), 122.36(9) ; \mathrm{P}(1)-\mathrm{C}(1)-\mathrm{Li}(1), 113.71(8)$.

Crystal data for $\left[\mathrm{Ph}_{2} \mathrm{PCHC}(\mathrm{Me}) \mathrm{CH}_{2} \mathrm{Li}(\mathrm{PMDETA})\right]$ 3a, $\mathrm{C}_{25} \mathrm{H}_{39} \mathrm{LiN}_{3} \mathrm{P}, 1953202$ (CCDC): $M=419.50$, clear orangish orange block, $0.676 \times 0.365 \times 0.313 \mathrm{~mm}^{3}$, space group (No. 14), $V=2541.17(15) \AA^{3}, Z=1, D_{\mathrm{c}}=1.096 \mathrm{~g} / \mathrm{cm}^{3}, F_{000}=912, X t a L A B$ 
Synergy, Dualflex, HyPix, MoK $\alpha$ radiation, $\lambda=0.71073 \AA, T=122.99(10) \mathrm{K}, 2 \theta_{\max }=61.8^{\circ}, 44535$ reflections collected, 6822 unique $\left(\mathrm{R}_{\text {int }}=0.1091\right)$. The structure was solved and refined using the programs SHELXT 2014/5 (Sheldrick, 2014) and ShelXL (Sheldrick, 2016) respectively. The program Olex $2^{4}$ was used as an interface to the SHELX programs and to prepare the figures. The PMDETA molecule was refined as disordered over two sites. Final $G o o F=1.047, R 1=0.0459, w R 2=0.1137$, $R$ indices based on 5200 reflections with $\mathrm{I}>2 \sigma(\mathrm{I})$ (refinement on $F^{2}$ ), 338 parameters, 0 restraints. Lp and absorption corrections applied, $\mu=0.123 \mathrm{~mm}^{-1}$.

3b:

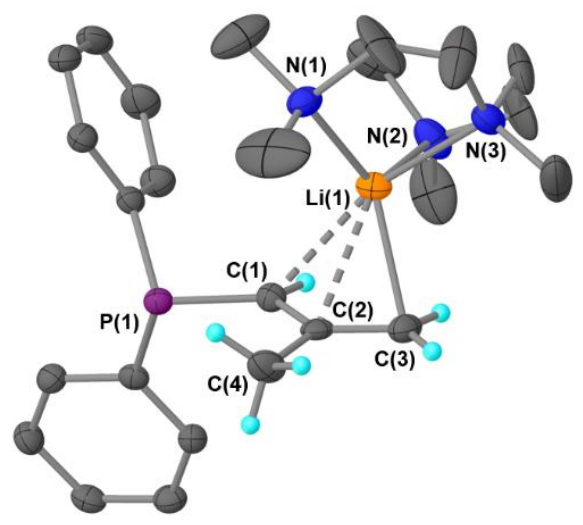

Figure S9. Molecular structure of $\left[\mathrm{Ph}_{2} \mathrm{PCHC}(\mathrm{Me}) \mathrm{CH}_{2} \mathrm{Li}(\mathrm{PMDETA})\right]$ 3b. Hydrogen atoms (except allylic) were omitted for clarity. Disorder in the PMDETA molecule was omitted for clarity. Thermal ellipsoids are shown at a $40 \%$ probability. Selected bond lengths $(\AA ̊)$ and angles $\left(^{\circ}\right)$ : $\mathrm{P}(1)-\mathrm{C}(1), 1.7620(13) ; \mathrm{C}(1)-\mathrm{C}(2), 1.4168(18)$; $\mathrm{C}(2)-\mathrm{C}(3), 1.3732(19) ; \mathrm{Li}(1)-\mathrm{C}(1), 2.494(3) ; \mathrm{Li}(1)-\mathrm{C}(2), 2.412(3)$; $\mathrm{Li}(1)-\mathrm{C}(3), 2.303(3) ; \mathrm{C}(2)-\mathrm{C}(4), 1.5165(19) ; \mathrm{P}(1)-\mathrm{C}(1)-\mathrm{C}(2), 123.19(10) ; \mathrm{P}(1)-\mathrm{C}(1)-\mathrm{Li}(1), 133.27(8)$.

Crystal data for $\left[\mathrm{Ph}_{2} \mathrm{PCHC}(\mathrm{Me}) \mathrm{CH}_{2} \mathrm{Li}(\mathrm{PMDETA})\right]$ 3b, $\mathrm{C}_{25} \mathrm{H}_{39} \mathrm{LiN}_{3} \mathrm{P}, 1953199$ (CCDC): $M=419.50$, clear light yellow block, $0.12 \times 0.10 \times 0.08 \mathrm{~mm}^{3}$, space group (No. 2), $V=1252.39(5) \AA^{3}, Z=2, D_{\mathrm{c}}=1.112 \mathrm{~g} / \mathrm{cm}^{3}, F_{000}=456$, XtaLAB Synergy, Dualflex, HyPix, MoK $\alpha$ radiation, $\lambda=0.71073 \AA$, $T=123.00(10) \mathrm{K}, 2 \theta_{\max }=61.4^{\circ}, 36038$ reflections collected, 6496 unique $\left(R_{\text {int }}=0.0913\right)$. The structure was solved and refined using the programs SHELXT 2014/5 (Sheldrick, 2014) and ShelXL (Sheldrick, 2016) respectively. The program Olex $2^{4}$ was used as an interface to the SHELX programs, and to prepare the figures. The PMDETA molecule was refined as disordered over two sites. Final $G o o F=1.057, R 1=0.0447, w R 2=0.1070, R$ indices based on 5059 reflections with $\mathrm{I}>2 \sigma(\mathrm{I})$ (refinement on $F^{2}$ ), 376 parameters, 0 restraints. Lp and absorption corrections applied, $\mu=0.125 \mathrm{~mm}^{-1}$. 


\section{Crystallographic data for 4:}

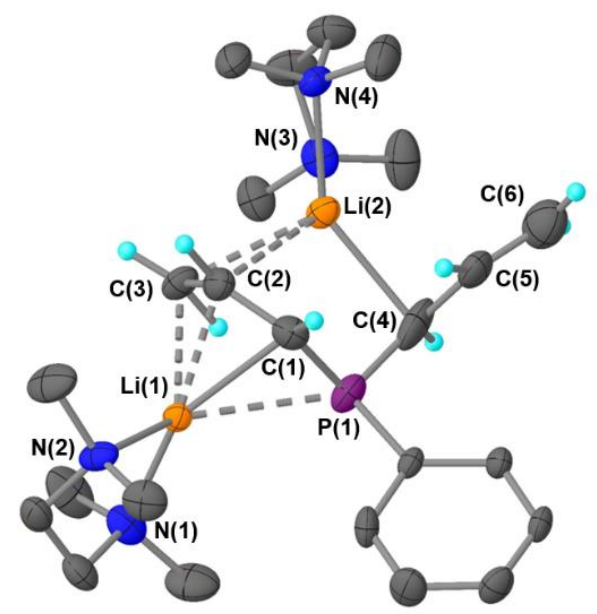

Figure S10. Molecular structure of $\left[\mathrm{PhP}\left\{\mathrm{CHCHCH}_{2} \mathrm{Li}(\mathrm{TMEDA})\right\}_{2}\right]$ 4. Hydrogen atoms (except allylic) were omitted for clarity. Disorder in one of the TMEDA molecules was omitted for clarity. Thermal ellipsoids are shown at a $40 \%$ probability. Selected bond lengths $(\AA)$ and angles $\left(^{\circ}\right)$ : $\mathrm{P}(1)-\mathrm{C}(1), 1.797(6) ; \mathrm{C}(1)-\mathrm{C}(2), 1.414(8) ; \mathrm{C}(2)-\mathrm{C}(3), 1.356(8) ; \mathrm{Li}(1)-\mathrm{P}(1), 2.778(8) ; \mathrm{Li}(1)-\mathrm{C}(1), 2.296(10)$; $\mathrm{Li}(1)-\mathrm{C}(2), 2.194(10) ; \mathrm{Li}(1)-\mathrm{C}(3), 2.335(9) ; \mathrm{Li}(2)-\mathrm{C}(2), 2.531(10) ; \mathrm{Li}(2)-\mathrm{C}(3), 2.520$ (11); $\mathrm{Li}(2)-\mathrm{C}(4), 2.289$ (11); $\mathrm{P}(1)-\mathrm{C}(4), 1.707(9)$; C(4)-C(5), 1.444(9); C(5)-C(6), 1.375(10); P(1)-C(1)-C(2), 119.2(5); $\mathrm{P}(1)-\mathrm{C}(4)-\mathrm{C}(5), 123.4(5)$.

Crystal data for $\left[\mathrm{PhP}\left\{\mathrm{CHCHCH}_{2} \mathrm{Li}(\mathrm{TMEDA})\right\}_{2}\right] 4, \mathrm{C}_{24} \mathrm{H}_{45} \mathrm{Li}_{2} \mathrm{~N}_{4} \mathrm{P}, 1953205$ (CCDC): $M=434.49$, clear orangish orange block, $0.133 \times 0.082 \times 0.053 \mathrm{~mm}^{3}$, space group (No. 14), $V=2745.0(7) \AA^{3}, Z=4, D_{\mathrm{c}}=1.046 \mathrm{~g} / \mathrm{cm}^{3}, F_{000}=944$, Bruker APEX-II $\mathrm{CCD}, \mathrm{MoK} \alpha$ radiation, $\lambda=0.71073 \AA$, $T=123 \mathrm{~K}, 2 \theta_{\max }=52.1^{\circ}, 25348$ reflections collected, 5382 unique $\left(\mathrm{R}_{\text {int }}=0.0912\right)$. The structure was solved and refined using the programs SHELXT 2014/5 (Sheldrick, 2014) and ShelXL (Sheldrick, 2016) respectively. The program Olex $2^{4}$ was used as an interface to the SHELX programs, and to prepare the figures. Final GooF= $1.055, R 1=0.1132, w R 2=0.2961, R$ indices based on 2788 reflections with $\mathrm{I}>2 \sigma(\mathrm{I})$ (refinement on $F^{2}$ ), 288 parameters, 0 restraints. Lp and absorption corrections applied, $\mu=0.116 \mathrm{~mm}^{-1}$. 


\section{Crystallographic data for 5:}

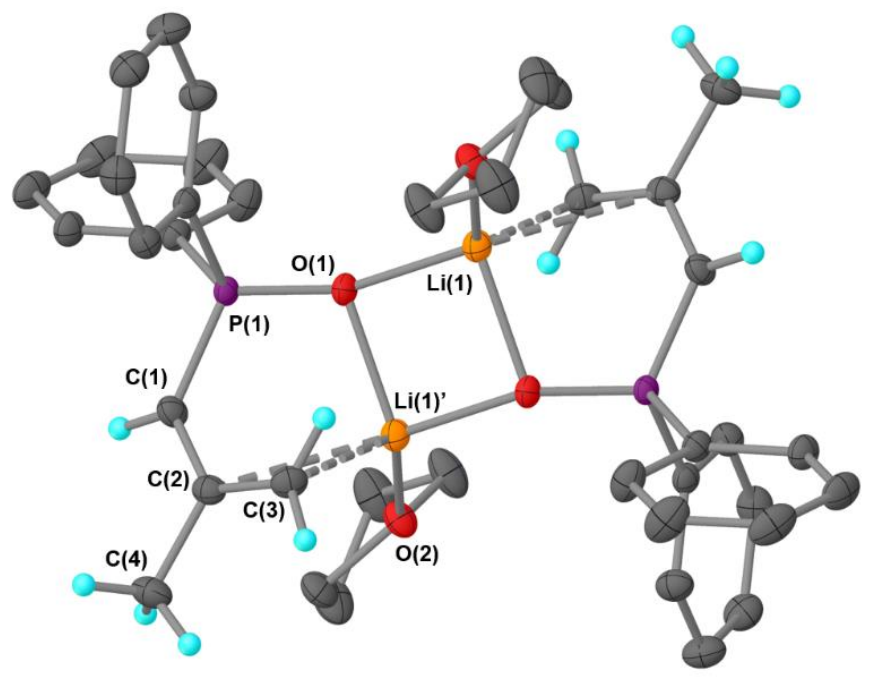

Figure S11. Molecular structure of $\left[\left\{\mathrm{Ph}_{2} \mathrm{P}(\mathrm{O}) \mathrm{CHC}(\mathrm{Me}) \mathrm{CH}_{2} \mathrm{Li}(\mathrm{THF})\right\}_{2}\right]$ 5. Hydrogen atoms (except allylic) were omitted for clarity. Thermal ellipsoids are shown at a $40 \%$ probability. Symmetry operator $\left(^{\circ}\right)=-\mathrm{x}, 1-\mathrm{y}, 1-\mathrm{z}$. Selected bond lengths $(\AA)$ and angles $\left(^{\circ}\right)$ : $\mathrm{P}(1)-\mathrm{C}(1), 1.7083(12) ; \mathrm{C}(1)-\mathrm{C}(2), 1.4259(16) ; \mathrm{C}(2)-\mathrm{C}(3), 1.3689(16) ; \mathrm{Li}(1)$ '- $\mathrm{C}(1), 2.890(3) ; \mathrm{Li}(1)$ ') $\mathrm{C}(2), 2.691(3) ; \mathrm{Li}(1)$ '- $\mathrm{C}(3)$, 2.403(3); $\mathrm{P}(1)-\mathrm{O}(1), 1.5240(10) ; \mathrm{Li}(1)-\mathrm{O}(1), 1.884(2)$; Li(1)'-O(1), 1.990(2); $\mathrm{P}(1)-\mathrm{C}(1)-\mathrm{C}(2), 124.11(9) ; \mathrm{P}(1)-\mathrm{O}(1)-\mathrm{Li}(1), 154.72(8)$; $\mathrm{P}(1)-\mathrm{O}(1)-\mathrm{Li}(1)^{\prime}, 108.31(7)$.

Crystal data for $\left[\left\{\mathrm{Ph}_{2} \mathrm{P}(\mathrm{O}) \mathrm{CHC}(\mathrm{Me}) \mathrm{CH}_{2} \mathrm{Li}(\mathrm{THF})\right\}_{2}\right] 5, \mathrm{C}_{40} \mathrm{H}_{48} \mathrm{Li}_{2} \mathrm{O}_{4} \mathrm{P}_{2}, 1953203$ (CCDC): $M=668.60$, clear yellowish orange needle, $0.1 \times 0.03 \times 0.03 \mathrm{~mm}^{3}$, space group (No. 2), $V=917.8(4) \AA^{3}, Z=1, D_{\mathrm{c}}=1.210 \mathrm{~g} / \mathrm{cm}^{3}, F_{000}=356$, MX1 Beamline Australian Synchrotron, ADSC Quantum 210r detector, Silicon Double Crystal monochromator, $\lambda=0.710756 \AA, T=100.00 \mathrm{~K}$, $2 \theta_{\max }=63.6^{\circ}, 26265$ reflections collected, 5291 unique $\left(\mathrm{R}_{\text {int }}=0.0488\right)$. The structure was solved and refined using the programs SHELXT 2014/5 (Sheldrick, 2014) and ShelXL (Sheldrick, 2016) respectively. The program Olex $2^{4}$ was used as an interface to the SHELX programs, and to prepare the figures. Final $G o o F=1.078, R 1=0.0414, w R 2=0.1057, R$ indices based on 4956 reflections with $\mathrm{I}>2 \sigma(\mathrm{I})$ (refinement on $F^{2}$ ), 222 parameters, 0 restraints. Lp and absorption corrections applied, $\mu=0.157 \mathrm{~mm}^{-1}$. 


\section{Crystallographic data for 6:}

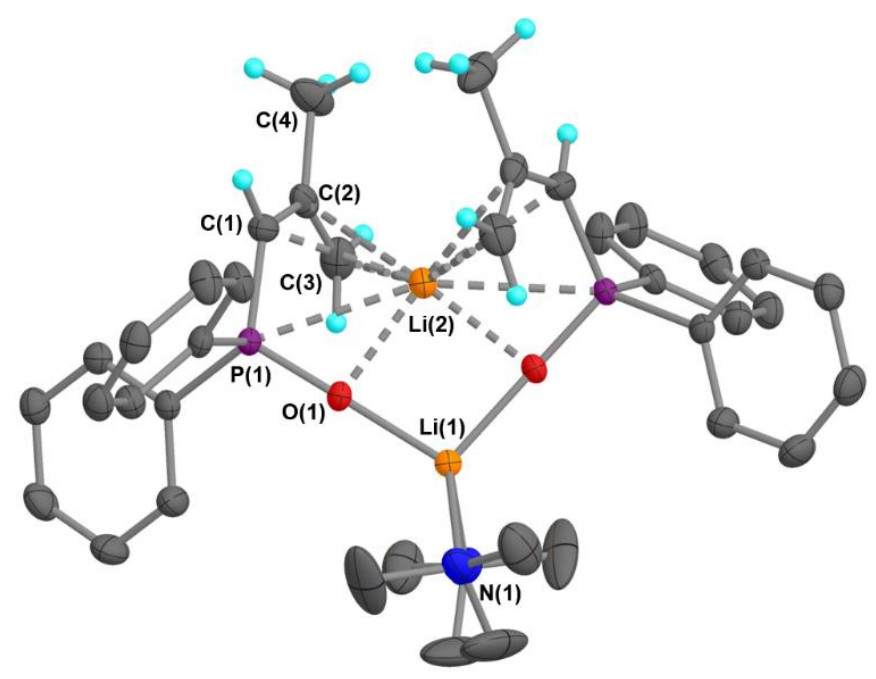

Figure S12. Molecular structure of $\left[\left\{\mathrm{Ph} 2 \mathrm{P}(\mathrm{O}) \mathrm{CHC}(\mathrm{Me}) \mathrm{CH}_{2} \mathrm{Li}_{2}(\mathrm{TMEDA})\right]\right.$ 6. Hydrogen atoms (except allylic) were omitted for clarity. Thermal ellipsoids are shown at a $40 \%$ probability. Symmetry operator (') $=1-\mathrm{x},+\mathrm{y}, 3 / 2$-z. Selected bond lengths $(\AA)$ and angles $\left(^{\circ}\right)$ : $\mathrm{P}(1)-\mathrm{C}(1), 1.7056(14) ; \mathrm{C}(1)-\mathrm{C}(2), 1.437(2) ; \mathrm{C}(2)-\mathrm{C}(3), 1.346(2) ; \mathrm{C}(2)-\mathrm{C}(4), 1.516(2) ; \mathrm{Li}(2)-\mathrm{C}(1), 2.435(2) ; \mathrm{Li}(2)-\mathrm{C}(2)$, 2.512(2); $\mathrm{Li}(2)-\mathrm{C}(3), 2.6713(18) ; \mathrm{Li}(2)-\mathrm{P}(1), 2.6570(8) ; \mathrm{P}(1)-\mathrm{O}(1), 1.5190(9) ; \mathrm{Li}(2)-\mathrm{O}(1), 2.043(3) ; \mathrm{Li}(1)-\mathrm{O}(1), 1.860(2) ; \mathrm{P}(1)-\mathrm{C}(1)-$ $\mathrm{C}(2), 122.56(11) ; \mathrm{P}(1)-\mathrm{O}(1)-\mathrm{Li}(1), 174.17(7) ; \mathrm{P}(1)-\mathrm{O}(1)-\mathrm{Li}(2), 95.36(9)$.

Crystal data for $\left[\left\{\mathrm{Ph}_{2} \mathrm{P}(\mathrm{O}) \mathrm{CHC}(\mathrm{Me}) \mathrm{CH}_{2} \mathrm{Li}\right\}_{2}\right.$ (TMEDA)] 6, $\mathrm{C}_{38} \mathrm{H}_{48} \mathrm{Li}_{2} \mathrm{~N}_{2} \mathrm{O}_{2} \mathrm{P}_{2}, 1953194$ (CCDC): $M=640.60$, clear orangish orange rect. Prism, $0.441 \times 0.178 \times 0.123 \mathrm{~mm}^{3}$, space group (No. 15), $V=3673.0(2) \AA^{3}, Z=4, D_{\mathrm{c}}=1.158 \mathrm{~g} / \mathrm{cm}^{3}, F_{000}=1368$, XtaLAB Synergy, Dualflex, HyPix, MoK $\alpha$ radiation, $\lambda=0.71073 \AA$, $T=122.99(10) \mathrm{K}, 2 \theta_{\max }=61.1^{\circ}, 18308$ reflections collected, 4506 unique $\left(\mathrm{R}_{\text {int }}=0.0314\right)$. The structure was solved and refined using the programs SHELXT 2014/5 (Sheldrick, 2014) and ShelXL (Sheldrick, 2016) respectively. The program Olex $2^{4}$ was used as an interface to the SHELX programs, and to prepare the figures. Final $G o o F=1.064, R 1=0.0411, w R 2=0.1046, R$ indices based on 3796 reflections with $\mathrm{I}>2 \sigma(\mathrm{I})$ (refinement on $F^{2}$ ), 212 parameters, 0 restraints. Lp and absorption corrections applied, $\mu=0.152 \mathrm{~mm}^{-1}$. 


\section{Crystallographic data for 7:}

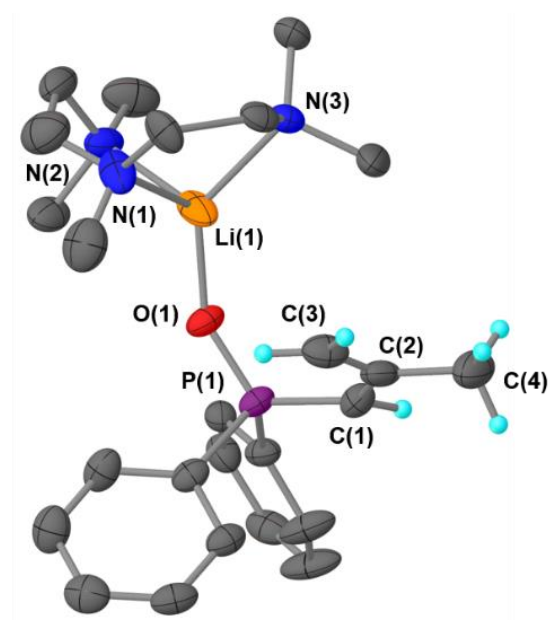

Figure S13. Monomeric structure of $\left[\mathrm{Ph} 2 \mathrm{P}(\mathrm{O}) \mathrm{CHC}(\mathrm{Me}) \mathrm{CH}_{2} \mathrm{Li}(\mathrm{PMDETA})\right]$ 7. Hydrogen atoms (except allylic) were omitted for clarity. Disorder in the PMDETA molecule was omitted for clarity. Thermal ellipsoids are shown at a $40 \%$ probability. Selected bond lengths $(\AA)$ and angles $\left({ }^{\circ}\right)$ : $\mathrm{P}(1)-\mathrm{C}(1), 1.7003(17) ; \mathrm{C}(1)-\mathrm{C}(2)$, 1.433(2); $\mathrm{C}(2)-\mathrm{C}(3), 1.361(3) ; \mathrm{C}(2)-\mathrm{C}(4), 1.496(3) ; \mathrm{P}(1)-\mathrm{O}(1)$, 1.5123(11); $\mathrm{Li}(1)-\mathrm{O}(1), 1.804(3) ; \mathrm{P}(1)-\mathrm{C}(1)-\mathrm{C}(2), 124.60(13) ; \mathrm{P}(1)-\mathrm{O}(1)-\mathrm{Li}(1), 147.03(14)$.

Crystal data for $\left[\mathrm{Ph}_{2} \mathrm{P}(\mathrm{O}) \mathrm{CHC}(\mathrm{Me}) \mathrm{CH}_{2} \mathrm{Li}(\mathrm{PMDETA})\right]$ 7, $\mathrm{C}_{25} \mathrm{H}_{39} \mathrm{LiN}_{3} \mathrm{OP}, 1953201$ (CCDC): $M=435.50$, clear orangish orange block, $0.25 \times 0.2 \times 0.2 \mathrm{~mm}^{3}$, space group (No. 2), $V=1296.15(6) \AA^{3}, Z=2, D_{\mathrm{c}}=1.116 \mathrm{~g} / \mathrm{cm}^{3}, F_{000}=472$, Bruker APEX-II CCD, MoK $\alpha$ radiation, $\lambda=0.71073 \AA, T=123 \mathrm{~K}, 2 \theta_{\max }=53.5^{\circ}, 34039$ reflections collected, 5504 unique $\left(\mathrm{R}_{\text {int }}=\right.$ 0.0274). The structure was solved and refined using the programs SHELXT 2014/5 (Sheldrick, 2014) and ShelXL (Sheldrick, 2016) respectively. The program Olex $2^{4}$ was used as an interface to the SHELX programs and to prepare the figures. The PMDETA molecule was refined as disordered over two sites. Final $G o o F=1.027, R 1=0.0427, w R 2=0.1101, R$ indices based on 4380 reflections with $\mathrm{I}>2 \sigma(\mathrm{I})$ (refinement on $F^{2}$ ), 400 parameters, 24 restraints. Lp and absorption corrections applied, $\mu$ $=0.126 \mathrm{~mm}^{-1}$. 


\section{Crystallographic data for 8:}

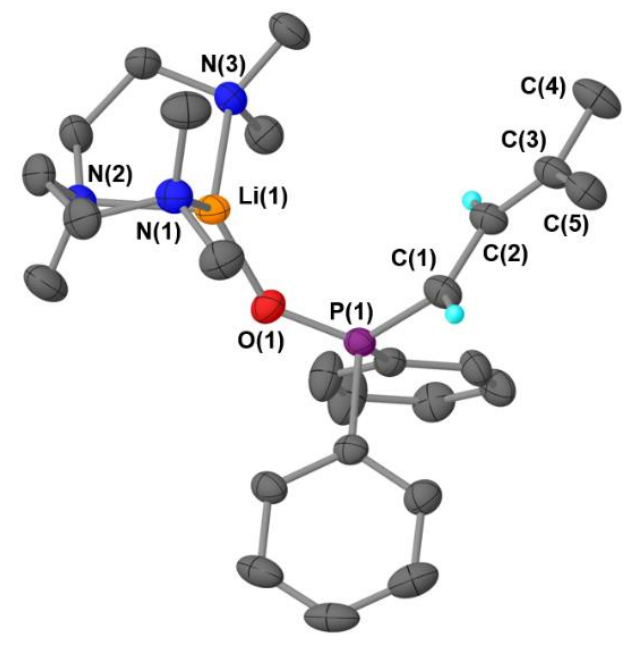

Figure S14. Monomeric structure of $\left[\mathrm{Ph}_{2} \mathrm{P}(\mathrm{O}) \mathrm{CHCHCMe}_{2} \mathrm{Li}(\mathrm{PMDETA})\right]$ 8. Hydrogen atoms (except allylic and including allyl-Me $\left.\mathrm{Me}_{2}\right)$ were omitted for clarity. Thermal ellipsoids are shown at a $40 \%$ probability. Selected bond lengths $(\AA)$ and angles $\left(^{\circ}\right): \mathrm{P}(1)-\mathrm{C}(1)$, 1.696(2); C(1)-C(2), 1.436(3); C(2)-C(3), 1.348(3); P(1)-O(1), 1.5197(15); $\mathrm{O}(1)-\mathrm{Li}(1), 1.801(4) ; \mathrm{P}(1)-\mathrm{C}(1)-\mathrm{C}(2), 124.59(18)$; $\mathrm{P}(1)-$ $\mathrm{Li}(1)-\mathrm{O}(1), 137.33(16)$.

Crystal data for $\left[\mathrm{Ph} 2 \mathrm{P}(\mathrm{O}) \mathrm{CHCHCMe}_{2} \mathrm{Li}(\mathrm{PMDETA})\right] \mathrm{8}, \mathrm{C}_{26} \mathrm{H}_{41} \mathrm{LiN}_{3} \mathrm{OP}, 1953197$ (CCDC): $M=449.53$, clear dark red block, $0.15 \times 0.1 \times 0.1 \mathrm{~mm}^{3}$, space group (No. 2), $V=1334.06(6) \AA^{3}, Z=2, D_{\mathrm{c}}=1.119 \mathrm{~g} / \mathrm{cm}^{3}, F_{000}=488$, XtaLAB Synergy, Dualflex, HyPix, CuK $\alpha$ radiation, $\lambda=1.54184 \AA, T=123.00(10) \mathrm{K}, 2 \theta_{\max }=154.5^{\circ}, 26617$ reflections collected, 5541 unique $\left(\mathrm{R}_{\text {int }}=\right.$ 0.1372). The structure was solved and refined using the programs SHELXT 2014/5 (Sheldrick, 2014) and ShelXL (Sheldrick, 2016) respectively. The program Olex $2^{4}$ was used as an interface to the SHELX programs and to prepare the figures. Final $G o o F=1.115, R 1=0.0777, w R 2=0.2150, R$ indices based on 4652 reflections with $\mathrm{I}>2 \sigma(\mathrm{I})$ (refinement on $F^{2}$ ), 308 parameters, 0 restraints. Lp and absorption corrections applied, $\mu=1.060 \mathrm{~mm}^{-1}$. 


\section{Crystallographic data for 9:}

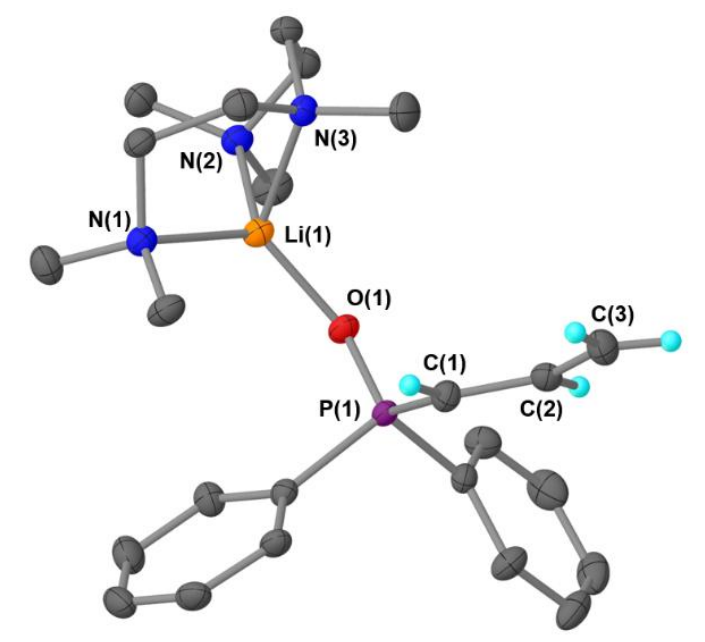

Figure S15. Monomeric structure of $\left[\mathrm{Ph}_{2} \mathrm{P}(\mathrm{O}) \mathrm{CHCHCH}_{2} \mathrm{Li}(\mathrm{PMDETA})\right]$ 9. Hydrogen atoms (except allylic) were omitted for clarity. Thermal ellipsoids are shown at a $40 \%$ probability. Selected bond lengths $(\AA)$ and angles $\left(^{\circ}\right)$ : $\mathrm{P}(1)-\mathrm{C}(1), 1.7073(16)$; $\mathrm{C}(1)-\mathrm{C}(2)$, 1.421(2); $\mathrm{C}(2)-\mathrm{C}(3), 1.356(2) ; \mathrm{P}(1)-\mathrm{O}(1), 1.5226(11) ; \mathrm{Li}(1)-\mathrm{O}(1), 1.837(3) ; \mathrm{P}(1)-\mathrm{C}(1)-\mathrm{C}(2), 122.34(12) ; \mathrm{P}(1)-\mathrm{O}(1)-\mathrm{Li}(1), 133.79(10)$.

Crystal data for $\left[\mathrm{Ph}_{2} \mathrm{P}(\mathrm{O}) \mathrm{CHCHCH}_{2} \mathrm{Li}(\mathrm{PMDETA})\right]$ 9, $\mathrm{C}_{24} \mathrm{H}_{37} \mathrm{LiN}_{3} \mathrm{OP}, 1953204$ (CCDC): $M=421.47$, clear orangish orange block, $0.13 \times 0.12 \times 0.07 \mathrm{~mm}^{3}$, space group (No. 2), $V=1210.1(5) \AA^{3}, Z=2, D_{\mathrm{c}}=1.157 \mathrm{~g} / \mathrm{cm}^{3}, F_{000}=456$, MX1 Beamline Australian Synchrotron, ADSC Quantum 210r detector, Silicon Double Crystal monochromator, $\lambda=0.710756 \AA, T=100.00 \mathrm{~K}$, $2 \theta_{\max }=63.5^{\circ}, 23828$ reflections collected, 6773 unique $\left(\mathrm{R}_{\text {int }}=0.0516\right)$. The structure was solved and refined using the programs SHELXT 2014/5 (Sheldrick, 2014) and ShelXL (Sheldrick, 2016) respectively. The program Olex $2^{4}$ was used as an interface to the SHELX programs and to prepare the figures. Final $G o o F=1.058, R 1=0.0508, w R 2=0.1239, R$ indices based on 5432 reflections with $\mathrm{I}>2 \sigma(\mathrm{I})$ (refinement on $F^{2}$ ), 287 parameters, 0 restraints. Lp and absorption corrections applied, $\mu$ $=0.133 \mathrm{~mm}^{-1}$. 


\section{NMR Spectra}

\begin{tabular}{|c|c|c|c|c|c|c|c|c|c|c|c|c|}
\hline & \multicolumn{9}{|c}{ P(III) complexes } & \multicolumn{5}{c|}{ P(V) complexes } \\
\hline Shift & $\mathbf{1}$ & $\mathbf{2}$ & $\mathbf{3}$ & $\mathbf{4}$ & $\mathbf{1 0}$ & $\mathbf{1 1}$ & $\mathbf{5}$ & $\mathbf{6}$ & $\mathbf{7}$ & $\mathbf{8}$ & $\mathbf{9}$ & $\mathbf{1 2}$ \\
\hline $\begin{array}{c}\text { solid-state } \\
\text { aggregation }\end{array}$ & dimer & dimer & $\begin{array}{c}\text { mono- } \\
\text { mer }\end{array}$ & $\begin{array}{c}\text { mono- } \\
\text { mer }\end{array}$ & - & - & dimer & $\begin{array}{c}\text { dimer } \\
\text { mono- } \\
\text { mer }\end{array}$ & $\begin{array}{c}\text { mono- } \\
\text { mer }\end{array}$ & $\begin{array}{c}\text { mono- } \\
\text { mer }\end{array}$ & - \\
\hline beta & 2.61 & 2.76 & 3.38 & 3.62 & 2.63 & 3.55 & $2.72^{\mathrm{a}}$ & 2.82 & 3.05 & 3.16 & 3.7 & 3.37 \\
\hline$\gamma 1$ & -6.45 & 6.46 & - & 6.95 & 6.37 & - & - & - & - & 6.24 & 6.88 & 6.63 \\
\hline$\gamma 2$ & - & - & 3.58 & 3.16 & - & 3.34 & $3.96^{\mathrm{a}}$ & 4.24 & 4.05 & - & 4.63 & 4.17 \\
\hline$\beta-\mathrm{Me}$ & - & - & 2.37 & - & - & 2.34 & $1.95^{\mathrm{a}}$ & 2.26 & 2.36 & - & - & - \\
\hline$\gamma-\mathrm{Me} 1$ & 1.9 & 1.81 & - & - & 2.07 & - & - & - & - & 2.17 & - & - \\
\hline$\gamma-\mathrm{Me} 2$ & 2.08 & 2.01 & - & - & 2.07 & - & - & - & - & 2.17 & - & - \\
\hline $7 \mathrm{Li}$ & -3.73 & -4.62 & -4.28 & -5.14 & -4.5 & -4.47 & $-3.82^{\mathrm{b}}$ & -4.18 & -3.99 & -3.94 & -3.94 & -3.67 \\
\hline $31 \mathrm{P}$ & -6.8 & -1.6 & -13.6 & -35.2 & -2.5 & $\begin{array}{c}27.6,- \\
19.7\end{array}$ & $37.0^{\mathrm{a}}$ & 33.3 & 26.1 & 28.9 & 29.2 & 38.8 \\
\hline
\end{tabular}

${ }^{a} \mathrm{NMR}$ collected in toluene $\left(\mathrm{C}_{7} \mathrm{H}_{8}\right)$ at $100{ }^{\circ} \mathrm{C}$. ${ }^{b} \mathrm{NMR}$ collected in toluene $\left(\mathrm{C}_{7} \mathrm{H}_{8}\right)$ at room temperature. 


\section{NMR spectra of $\left[\left\{\mathrm{Ph}_{2} \mathrm{PCHCHCMe} 2 \mathrm{Li}\left(\mathrm{Et}_{2} \mathrm{O}\right)\right\}_{2}\right] 1$ :}

${ }^{1} \mathrm{H}$ NMR $\left(\mathrm{C}_{6} \mathrm{D}_{6}, 400.20 \mathrm{MHz}\right): \delta 0.82\left(\mathrm{t},{ }^{3} \mathrm{~J}_{\mathrm{H}-\mathrm{H}}=7.1 \mathrm{~Hz}, 6 \mathrm{H}, \mathrm{Et}_{2} \mathrm{O}-\mathrm{Me}\right), 1.90(\mathrm{~s}, 3 \mathrm{H},-\mathrm{CH}=\mathrm{CMeMe}(\operatorname{trans})), 2.08(\mathrm{~s}, 3 \mathrm{H},-$ $\mathrm{CH}=\mathrm{CMeMe}(\mathrm{cis})), 2.61\left(\mathrm{dd},{ }^{3} J_{\mathrm{H}-\mathrm{H}}=12.7 \mathrm{~Hz},{ }^{2} J_{\mathrm{H}-\mathrm{P}}=5.1 \mathrm{~Hz}, 1 \mathrm{H}, \mathrm{P}-\mathrm{CH}-\right), 3.04\left(\mathrm{q},{ }^{3} J_{\mathrm{H}-\mathrm{H}}=7.1 \mathrm{~Hz}, 4 \mathrm{H}, \mathrm{Et}_{2} \mathrm{O}-\mathrm{CH}_{2}\right) 6.45\left(\mathrm{t},{ }^{3} J_{\mathrm{H}-}\right.$ $\left.\mathrm{P}=14.3 \mathrm{~Hz},{ }^{3} J_{\mathrm{H}-\mathrm{H}}=12.7 \mathrm{~Hz}, 1 \mathrm{H},-\mathrm{CH}=\right), 7.06(\mathrm{~m}, 2 \mathrm{H}, p-\mathrm{H}), 7.14(\mathrm{~m}, 4 \mathrm{H}, m-\mathrm{H}), 7.65(\mathrm{~m}, 4 \mathrm{H}, o-\mathrm{H})$.

${ }^{7} \mathrm{Li}$ NMR (C6D6, $\left.155.53 \mathrm{MHz}\right): \delta-3.73$.

${ }^{13} \mathrm{C}$ NMR $\left(\mathrm{C}_{6} \mathrm{D}_{6}, 100.6 \mathrm{MHz}\right): \delta 14.46\left(\mathrm{~s}, \mathrm{Et}_{2} \mathrm{O}-\mathrm{CH}_{3}\right), 18.93\left(\mathrm{~d},{ }^{4} J_{\mathrm{C}-\mathrm{P}}=2.1 \mathrm{~Hz},=\mathrm{CMeMe}(\operatorname{trans})\right), 26.57$ (s, $\left.-\mathrm{CH}=\mathrm{CMeMe}(\mathrm{cis})\right)$, 34.64 (m, P-CH-), 66.29 (s, $\left.\mathrm{Et}_{2} \mathrm{O}-\mathrm{CH}_{2}\right), 102.93\left(\mathrm{~m},=\mathrm{CMe}_{2}\right), 127.05(\mathrm{~s}, p-\mathrm{C}), 128.1(\mathrm{~m}, m-\mathrm{C}), 132.36\left(\mathrm{~d},{ }^{2} J_{\mathrm{C}-\mathrm{P}}=14.0 \mathrm{~Hz}, o-\right.$ C), $132.53(\mathrm{~m},-\mathrm{CH}=), 146.34(\mathrm{~m}, i-\mathrm{C})$.

${ }^{31} \mathrm{P}\left\{{ }^{1} \mathrm{H}\right\}$ NMR $\left(\mathrm{C}_{6} \mathrm{D}_{6}, 162.0 \mathrm{MHz}\right): \delta-6.8$.<smiles>CCOC1C(C(C)=C(C)C)[PH](c2ccccc2)(c2ccccc2)C(C(C)=C(C)C)[IH](c2ccccc2)(c2ccccc2)C1OCC</smiles>

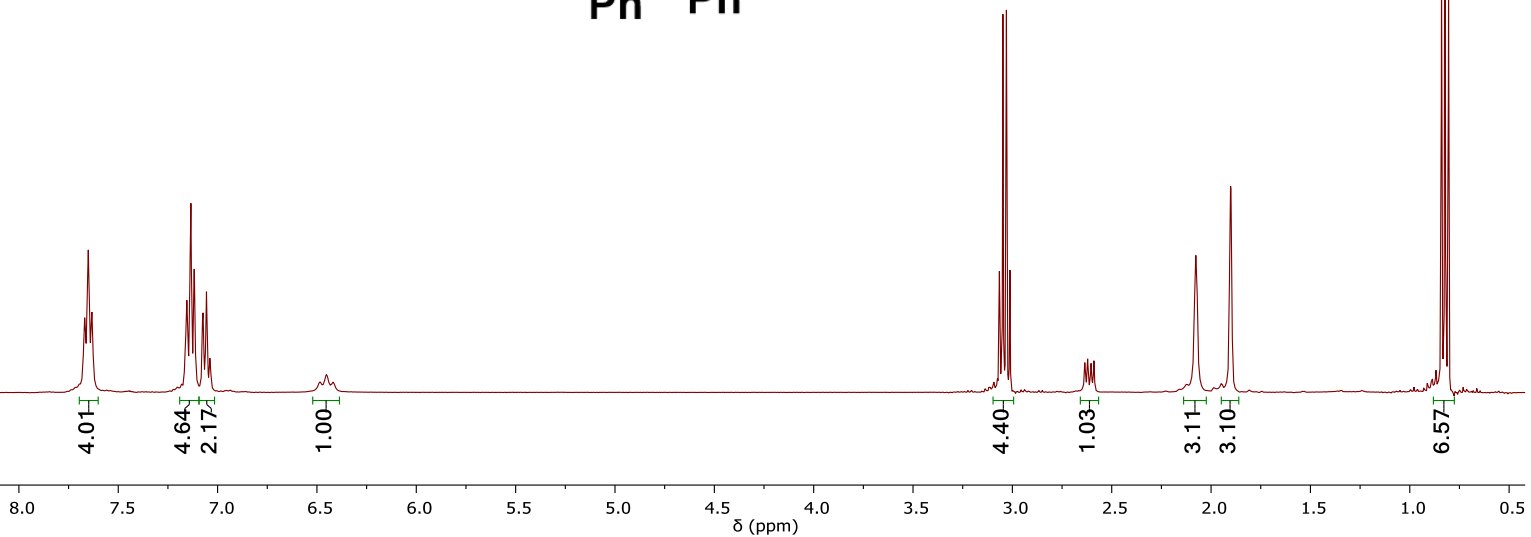

Figure S16. ${ }^{1} \mathrm{H}-\mathrm{NMR}\left(\mathrm{C}_{6} \mathrm{D}_{6}\right)$ of $\left[\left\{\mathrm{Ph}_{2} \mathrm{PCHCHCMe} \mathrm{Li}\left(\mathrm{Et}_{2} \mathrm{O}\right)\right\}_{2}\right] 1$ 


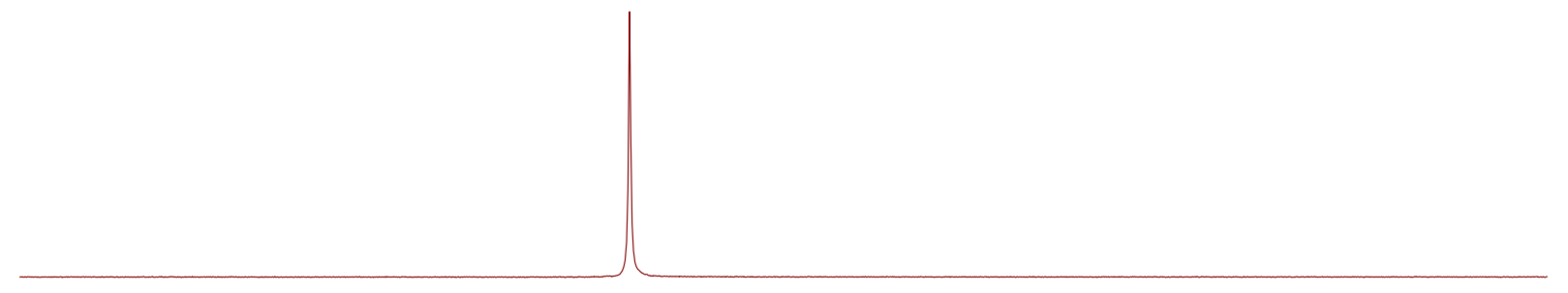

\begin{tabular}{llllllllllllllllllllllllllllllllll}
\hline 14 & 12 & 10 & 8 & 6 & 4 & 2 & 0 & -2 & -4 & -6 & -8 & -10 & -12 & -14 & -16 & -18 & -20 & -22 & -24 & -26 & -28 & -30 & -3 \\
\hline
\end{tabular}

Figure S17. ${ }^{7} \mathrm{Li}-\mathrm{NMR}\left(\mathrm{C}_{6} \mathrm{D}_{6}\right)$ of $\left[\left\{\mathrm{Ph}_{2} \mathrm{PCHCHCMe}_{2} \mathrm{Li}\left(\mathrm{Et}_{2} \mathrm{O}\right)\right\}_{2}\right] 1$

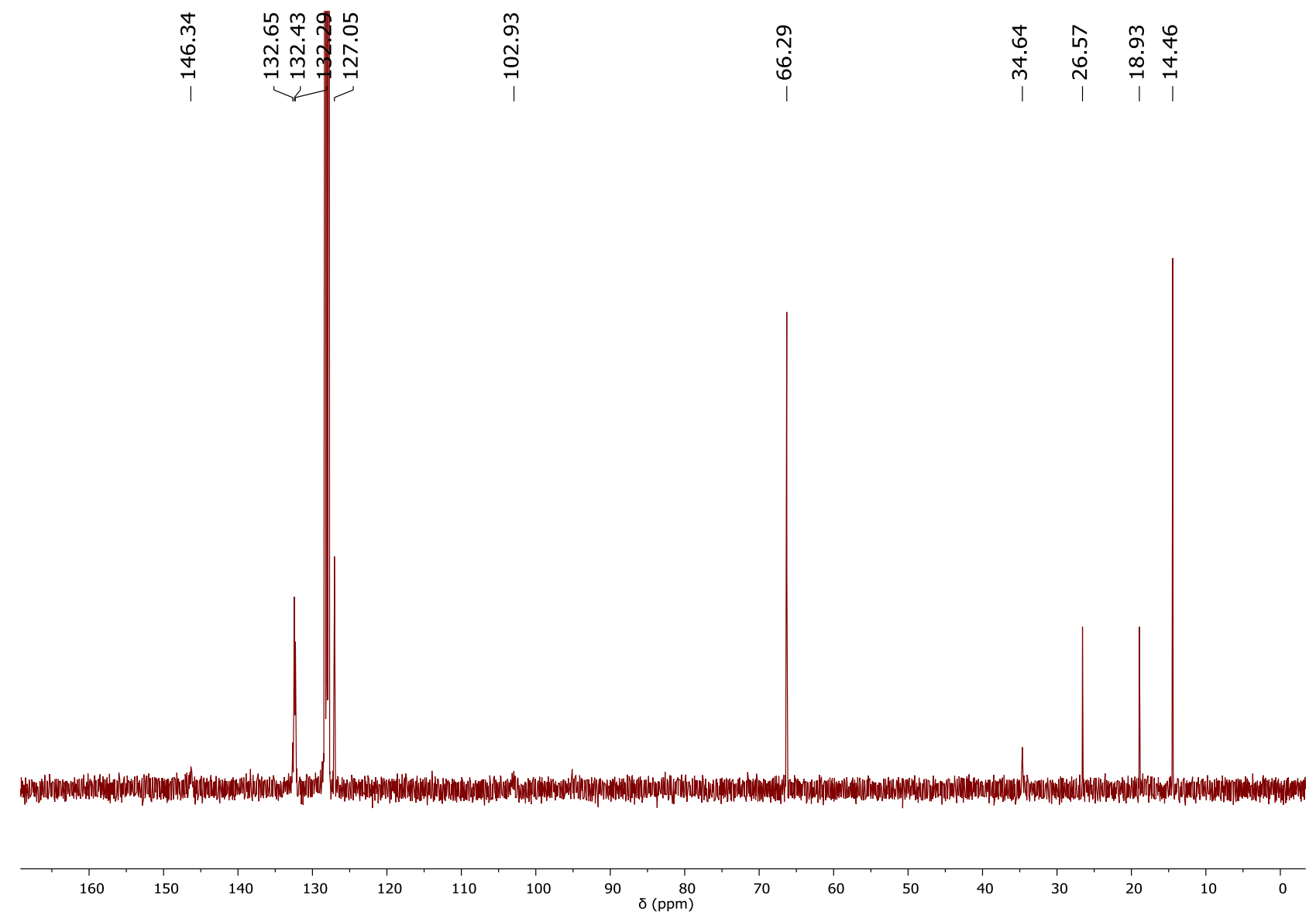

Figure S18. ${ }^{13} \mathrm{C}-\mathrm{NMR}\left(\mathrm{C}_{6} \mathrm{D}_{6}\right)$ of $\left[\left\{\mathrm{Ph}_{2} \mathrm{PCHCHCMe}_{2} \mathrm{Li}_{2}\left(\mathrm{Et}_{2} \mathrm{O}\right)\right\}_{2}\right] 1$ 


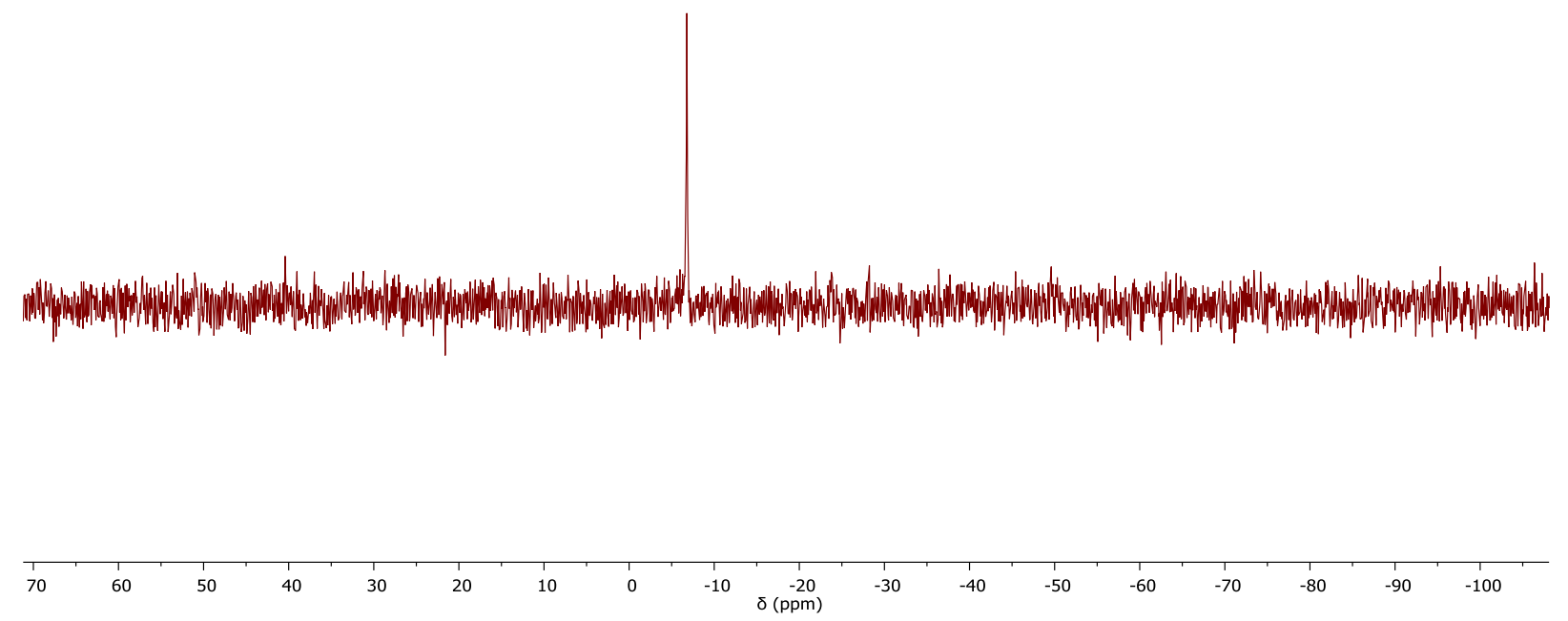

Figure S19. ${ }^{31} \mathrm{P}\{\mathrm{H}\}-\mathrm{NMR}\left(\mathrm{C}_{6} \mathrm{D}_{6}\right)$ of $\left[\left\{\mathrm{Ph}_{2} \mathrm{PCHCHCMe} 2 \mathrm{Li}\left(\mathrm{Et}_{2} \mathrm{O}\right)\right\}_{2}\right] 1$

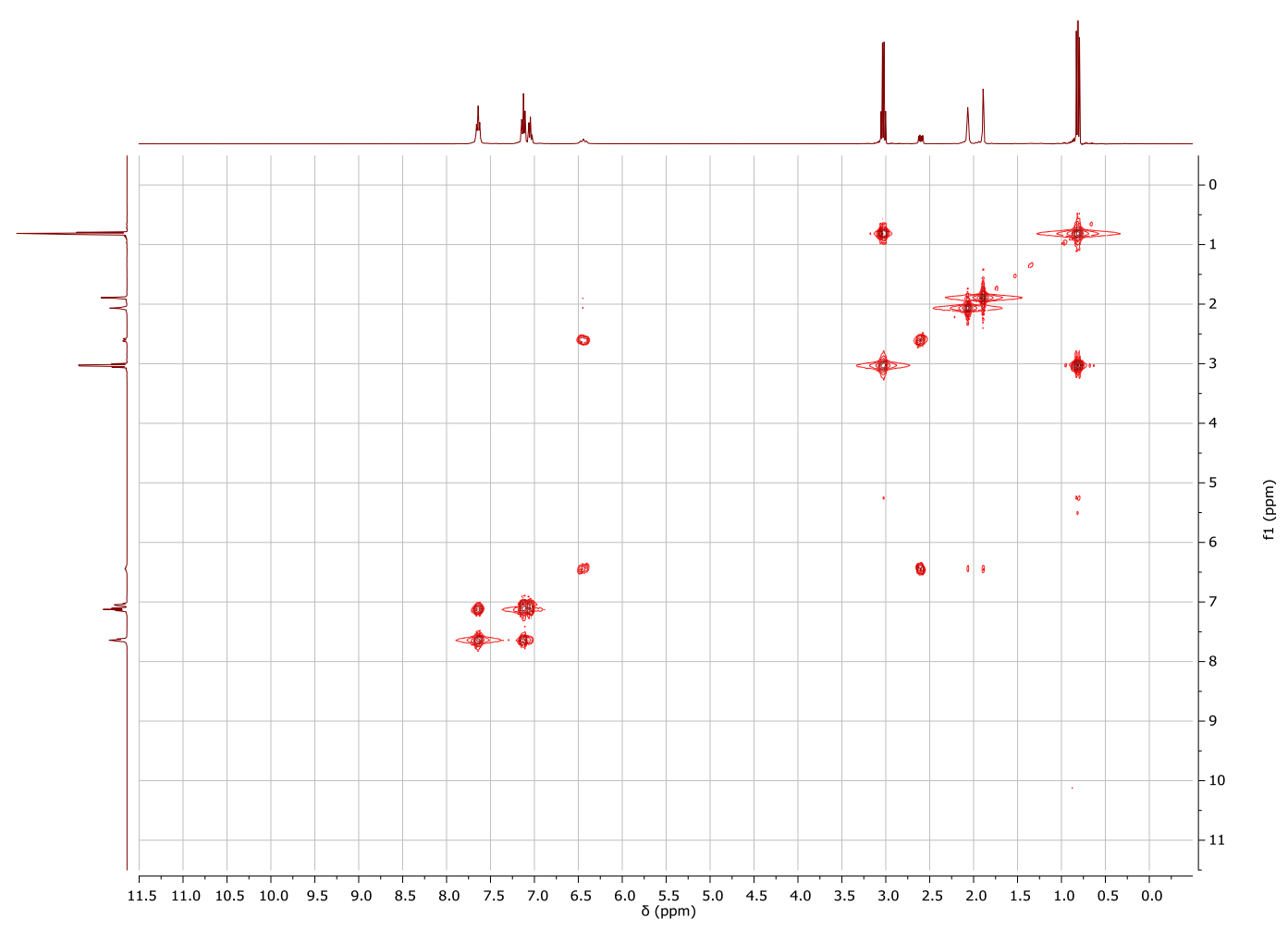

Figure S20. ${ }^{1} \mathrm{H}-{ }^{1} \mathrm{H}$ COSY-NMR $\left(\mathrm{C}_{6} \mathrm{D}_{6}\right)$ of $\left[\left\{\mathrm{Ph}_{2} \mathrm{PCHCHCMe}_{2} \mathrm{Li}\left(\mathrm{Et}_{2} \mathrm{O}\right)\right\}_{2}\right] \mathbf{1}$ 


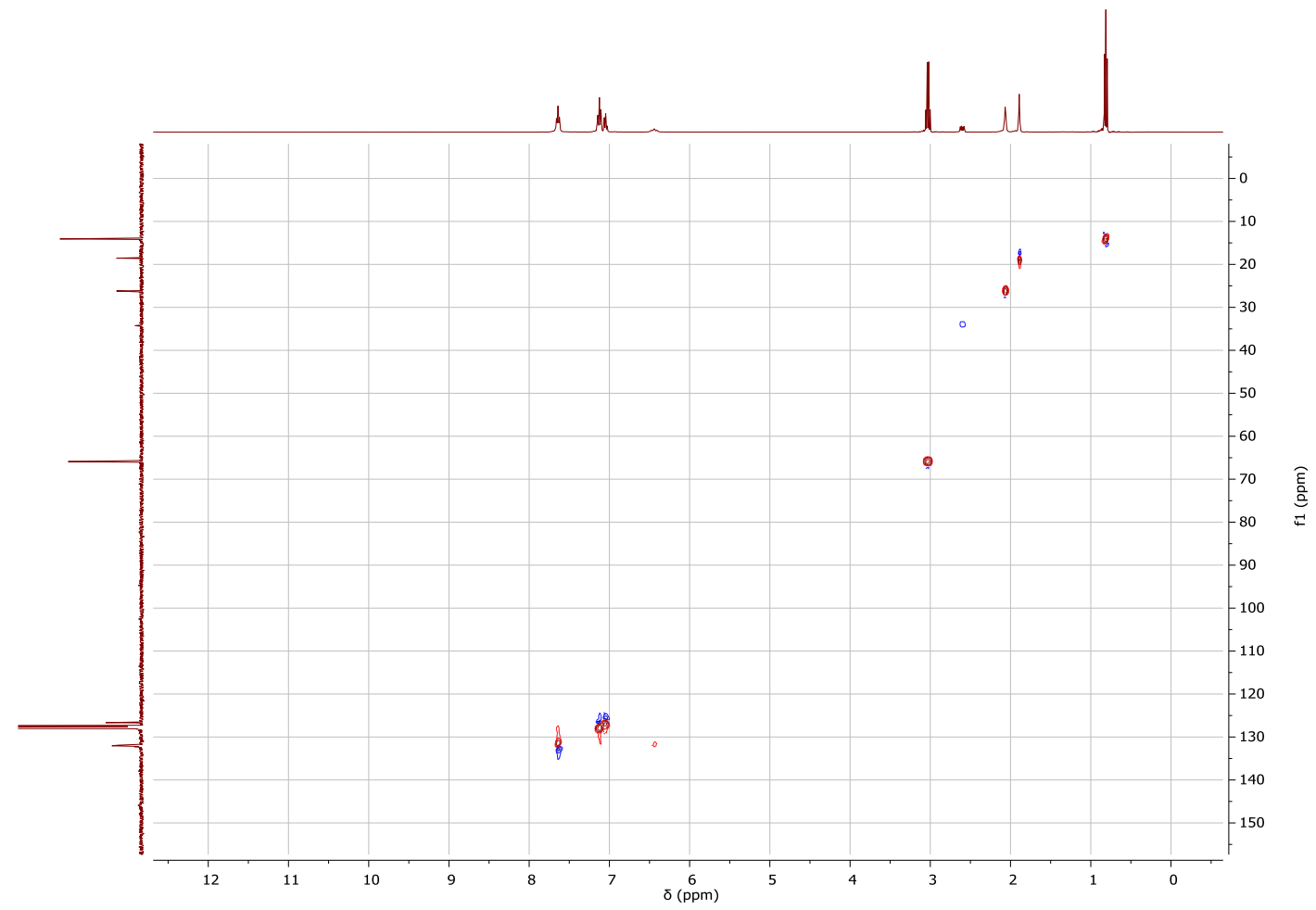

Figure S21. HMQC-NMR $\left(\mathrm{C}_{6} \mathrm{D}_{6}\right)$ of $\left[\left\{\mathrm{Ph}_{2} \mathrm{PCHCHCMe} 2 \mathrm{Li}\left(\mathrm{Et}_{2} \mathrm{O}\right)\right\}_{2}\right] \mathbf{1}$ 


\section{NMR spectra of $\left[\left\{\mathrm{Ph}_{2} \mathrm{PCHCHCMe} 2 \mathrm{Li}(\mathrm{TMEDA})\right\}_{2}\right]$ 2:}

${ }^{1} \mathrm{H}$ NMR $\left(\mathrm{C}_{6} \mathrm{D}_{6}, 400.20 \mathrm{MHz}\right): \delta 1.46$ (s, 4H, TMEDA-CH$), 1.66$ (s, 12H, TMEDA-CH 3 ), 1.81 (s, 3H, -CH=CMeMe(trans)), $2.01(\mathrm{~s}, 3 \mathrm{H},-\mathrm{CH}=\mathrm{CMeMe}(\mathrm{cis})), 2.76\left(\mathrm{dd},{ }^{3} J_{\mathrm{H}-\mathrm{H}}=12.8 \mathrm{~Hz},{ }^{2} J_{\mathrm{H}-\mathrm{P}}=7.6 \mathrm{~Hz}, 1 \mathrm{H}, \mathrm{P}-\mathrm{CH}-\right), 6.46\left(\mathrm{ddhept},{ }^{3} J_{\mathrm{H}-\mathrm{H}}=14.9\right.$ $\left.\mathrm{Hz},{ }^{3} J_{\mathrm{H}-\mathrm{H}}=12.8 \mathrm{~Hz},{ }^{4} J_{\mathrm{H}-\mathrm{H}}=1.0 \mathrm{~Hz}, 1 \mathrm{H},-\mathrm{CH}=\right), 7.07(\mathrm{~m}, 2 \mathrm{H}, p-\mathrm{H}), 7.19(\mathrm{~m}, 4 \mathrm{H}, m-\mathrm{H}), 7.84(\mathrm{~m}, 4 \mathrm{H}, o-\mathrm{H})$.

${ }^{7} \mathrm{Li}$ NMR $\left(\mathrm{C}_{6} \mathrm{D}_{6}, 155.53 \mathrm{MHz}\right): \delta-4.62$.

${ }^{13} \mathrm{C}$ NMR $\left(\mathrm{C}_{6} \mathrm{D}_{6}, 100.6 \mathrm{MHz}\right): \delta 18.76$ (s, -CH=CMeMe(trans)), 26.49 (s, -CH=CMeMe(cis)), 42.20 (s, P-CH-), 45.28 (s, TMEDA-Me), 56.22 (s, TMEDA-CH 2$), 91.63\left(\mathrm{~d},{ }^{3} J_{\mathrm{C}-\mathrm{P}}=24.5 \mathrm{~Hz},=\mathrm{CMe}_{2}\right), 126.04(\mathrm{~s}, p-\mathrm{C}), 127.74(\mathrm{~m}, m-\mathrm{C}), 132.43\left(\mathrm{~d},{ }^{2} J_{\mathrm{C}}\right.$ $\mathrm{P}=17.6 \mathrm{~Hz}, o-\mathrm{C}), 137.58\left(\mathrm{~d},{ }^{2} J_{\mathrm{C}-\mathrm{P}}=46.3 \mathrm{~Hz},-\mathrm{CH}=\right), 150.92\left(\mathrm{~d},{ }^{1} J_{\mathrm{C}-\mathrm{P}}=13.4 \mathrm{~Hz}, i-\mathrm{C}\right)$.

${ }^{31} \mathrm{P}\left\{{ }^{1} \mathrm{H}\right\}$ NMR $\left(\mathrm{C}_{6} \mathrm{D}_{6}, 162.0 \mathrm{MHz}\right): \delta-1.6$.

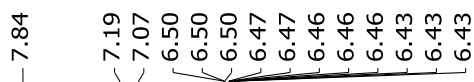

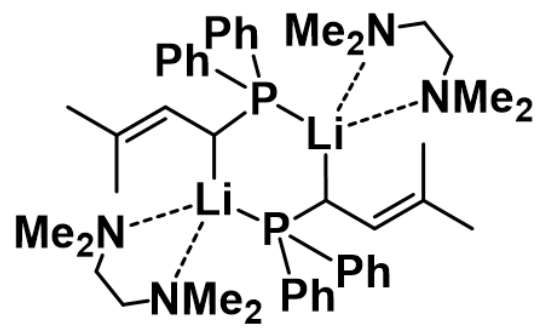

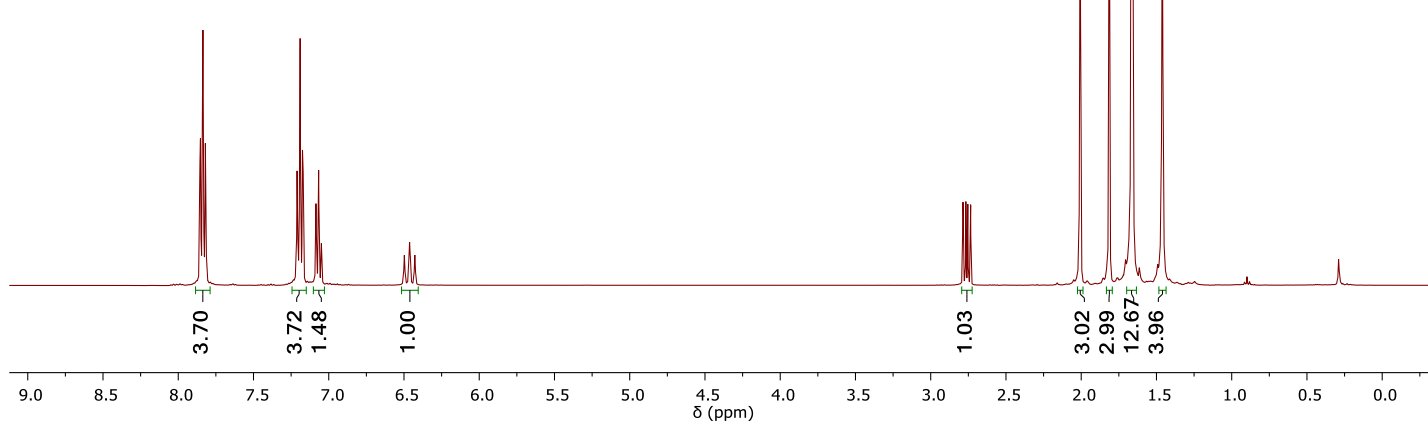

Figure S22. ${ }^{1} \mathrm{H}-\mathrm{NMR}\left(\mathrm{C}_{6} \mathrm{D}_{6}\right)$ of $\left[\left\{\mathrm{Ph}_{2} \mathrm{PCHCHCMe} 2 \mathrm{Li}(\mathrm{TMEDA})\right\}_{2}\right] 2$ 


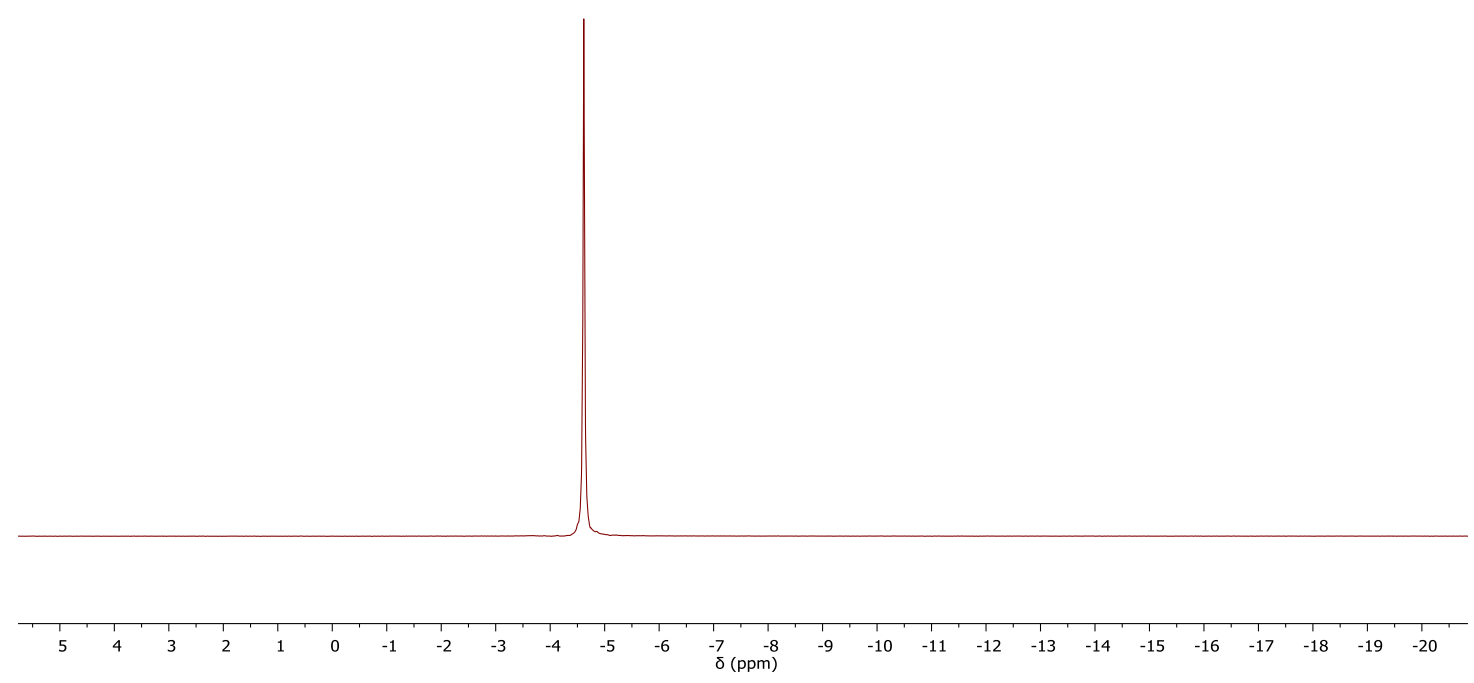

Figure S23. ${ }^{7} \mathrm{Li}-\mathrm{NMR}\left(\mathrm{C}_{6} \mathrm{D}_{6}\right)$ of $\left[\left\{\mathrm{Ph}_{2} \mathrm{PCHCHCMe} 2 \mathrm{Li}(\mathrm{TMEDA})\right\}_{2}\right] 2$

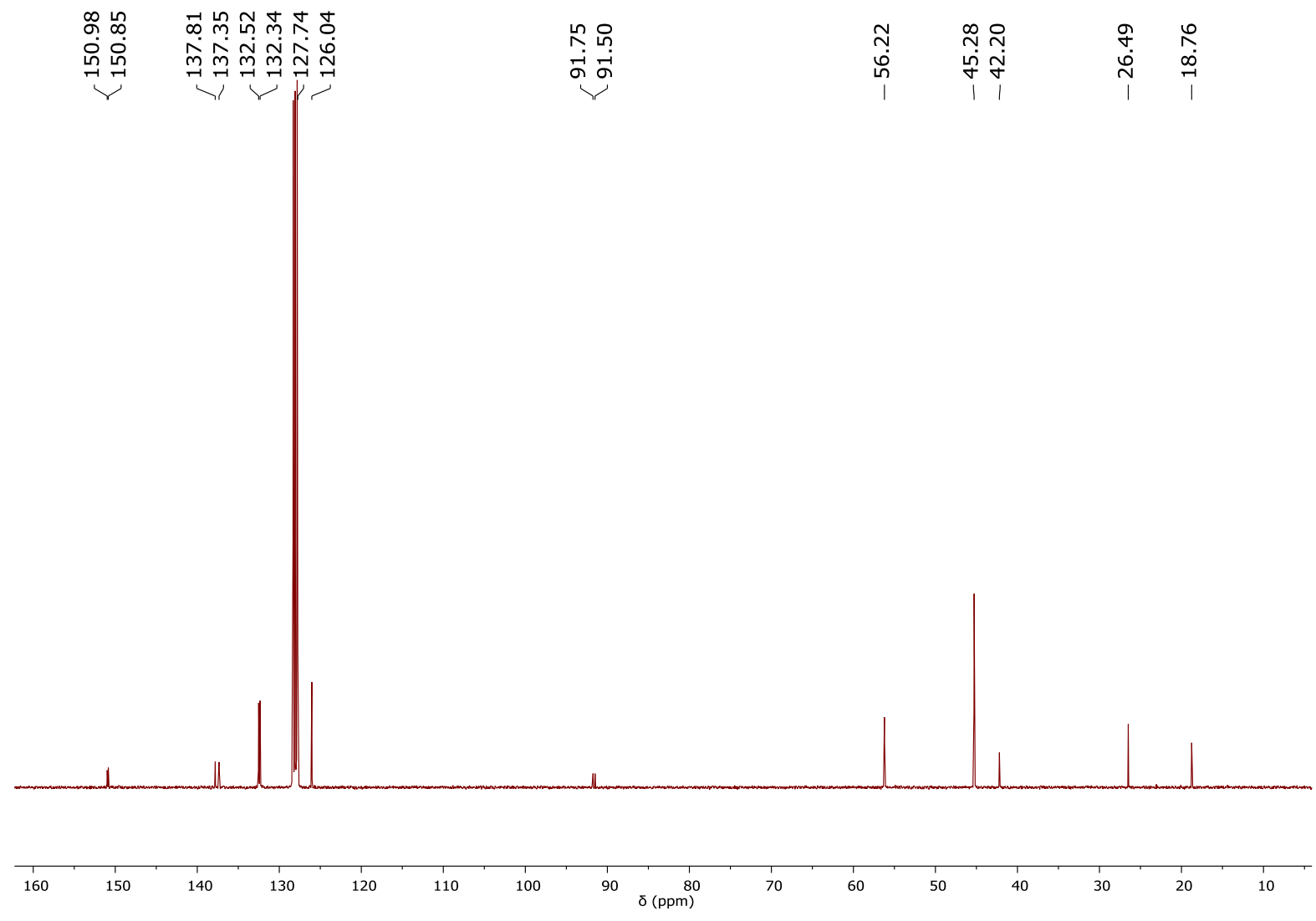

Figure S24. ${ }^{13} \mathrm{C}-\mathrm{NMR}\left(\mathrm{C}_{6} \mathrm{D}_{6}\right)$ of $\left[\left\{\mathrm{Ph}_{2} \mathrm{PCHCHCMe}_{2} \mathrm{Li}(\mathrm{TMEDA})\right\}_{2}\right] 2$ 


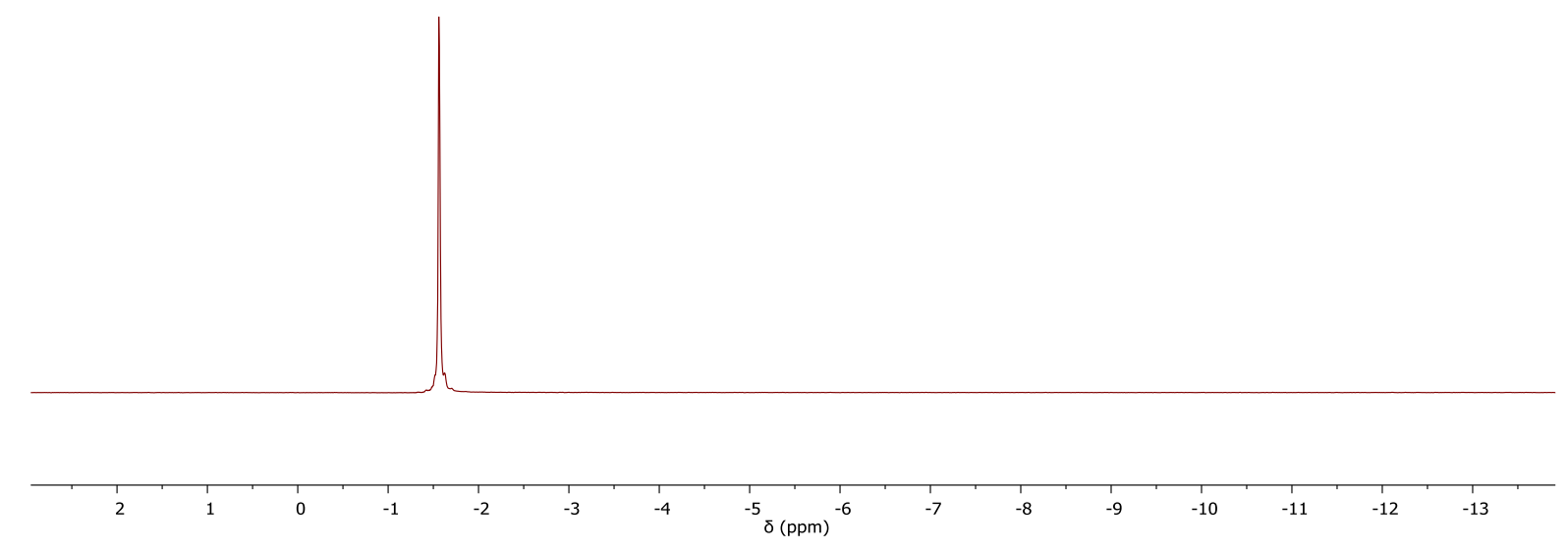

Figure S25. ${ }^{31} \mathrm{P}\{\mathrm{H}\}-\mathrm{NMR}\left(\mathrm{C}_{6} \mathrm{D}_{6}\right)$ of $\left[\left\{\mathrm{Ph}_{2} \mathrm{PCHCHCMe} 2 \mathrm{Li}(\mathrm{TMEDA})\right\}_{2}\right] 2$

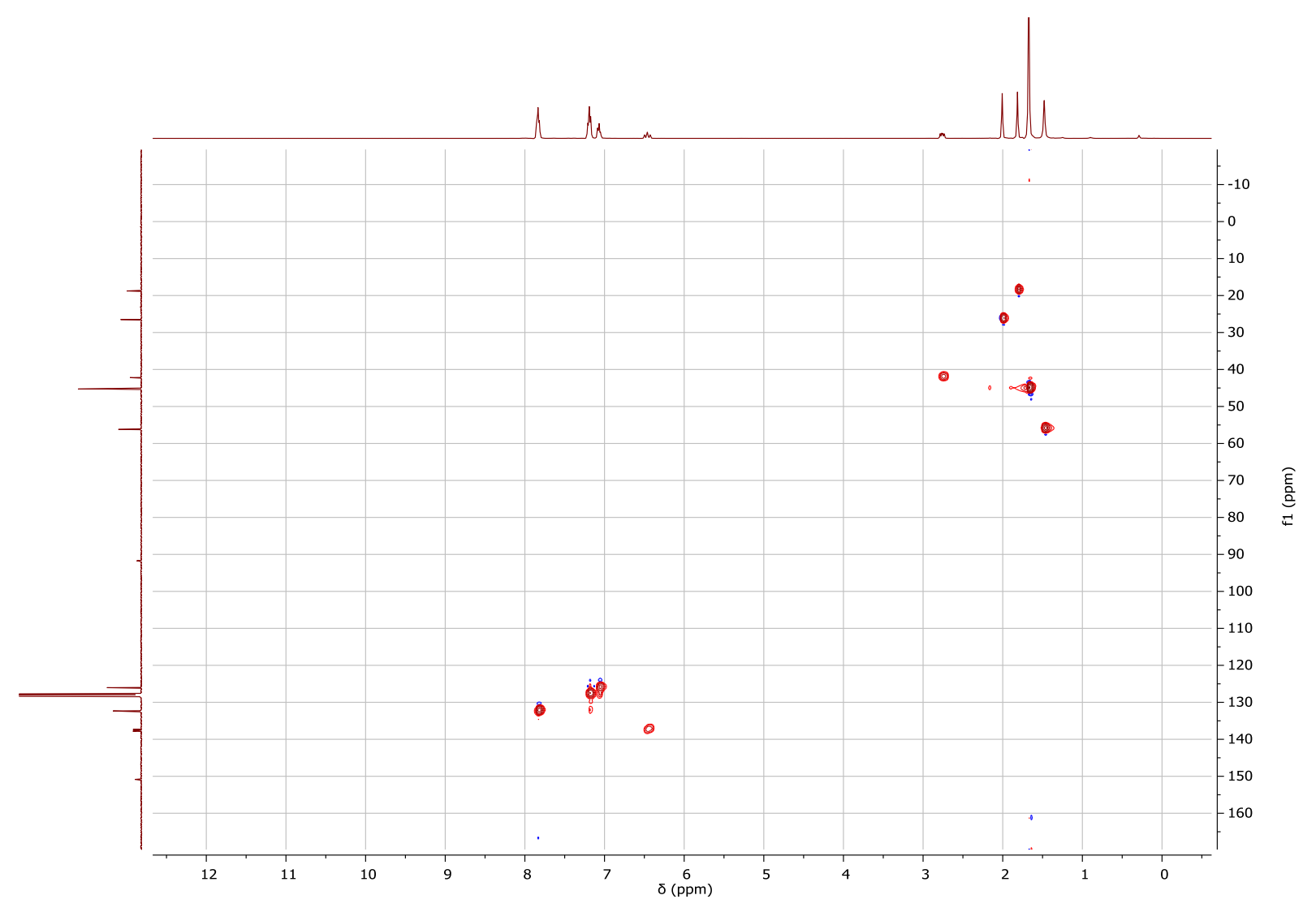

Figure S26. HMQC-NMR $\left(\mathrm{C}_{6} \mathrm{D}_{6}\right)$ of $\left[\left\{\mathrm{Ph}_{2} \mathrm{PCHCHCMe} \mathrm{Li}(\mathrm{TMEDA})\right\}_{2}\right] 2$ 


\section{NMR spectra of [Ph 2 PCHC(Me)CH $2 \mathrm{Li}(\mathrm{PMDETA})] 3$ (3a and 3b):}

${ }^{1} \mathrm{H}$ NMR $\left(\mathrm{C}_{6} \mathrm{D}_{6}, 400.20 \mathrm{MHz}\right): \delta 1.72-1.96(\mathrm{~m}, 23 \mathrm{H}, \mathrm{PMDETA}) 2.37\left(\mathrm{~m},{ }^{3} \mathrm{~J}_{\mathrm{H}-\mathrm{H}}=1.3 \mathrm{~Hz}, 3 \mathrm{H},-\mathrm{C}(\mathrm{Me})=\right), 3.20(\mathrm{br}-\mathrm{s}, 1 \mathrm{H},-$ $\mathrm{C}(\mathrm{Me})=\mathrm{CHH}), 3.38\left(\mathrm{~d},{ }^{2} J_{\mathrm{H}-\mathrm{P}}=7.65 \mathrm{~Hz}, \mathrm{P}-\mathrm{CH}-\right), 3.58(\mathrm{~s}, 1 \mathrm{H},-\mathrm{C}(\mathrm{Me})=\mathrm{CHH}), 7.06(\mathrm{~m}, 2 \mathrm{H}, p-\mathrm{H}), 7.19(\mathrm{~m}, 4 \mathrm{H}, m-\mathrm{H}), 7.82(\mathrm{~m}$, $4 \mathrm{H}, o-\mathrm{H})$.

${ }^{7} \mathrm{Li}$ NMR $\left(\mathrm{C}_{6} \mathrm{D}_{6}, 155.53 \mathrm{MHz}\right): \delta-4.28$.

${ }^{13} \mathrm{C}$ NMR ( $\left.\mathrm{C}_{6} \mathrm{D}_{6}, 100.6 \mathrm{MHz}\right): \delta 25.20$ (s, -C(Me)=), 44.76 (s, PMDETA), 45.86 (s, PMDETA), 53.68 (s, PMDETA), 54.87 (s, P-CH-), 57.30 (s, PMDETA), $69.43\left(\mathrm{~m},=\mathrm{CH}_{2}\right), 126.09$ (s, $p$-C), $127.72\left(\mathrm{~d},{ }^{3} J_{\mathrm{C}-\mathrm{P}}=5.6 \mathrm{~Hz}, m-\mathrm{C}\right), 132.85\left(\mathrm{~d},{ }^{2} J_{\mathrm{C}-\mathrm{P}}=17.7\right.$ $\mathrm{Hz}, o-\mathrm{C}), 150.21(\mathrm{~m},-\mathrm{C}(\mathrm{Me})=), 157.59\left(\mathrm{~d},{ }^{1} J_{\mathrm{C}-\mathrm{P}}=26.2 \mathrm{~Hz}, i-\mathrm{C}\right)$.

${ }^{31} \mathrm{P}\left\{{ }^{1} \mathrm{H}\right\}$ NMR $\left(\mathrm{C}_{6} \mathrm{D}_{6}, 162.00 \mathrm{MHz}\right): \delta-13.6$.

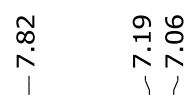

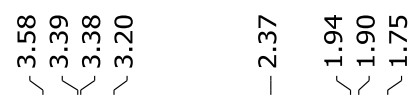<smiles>C=C(C)C([Al](NCCN(C)C)N(C)CCN)P(c1ccccc1)c1ccccc1</smiles>

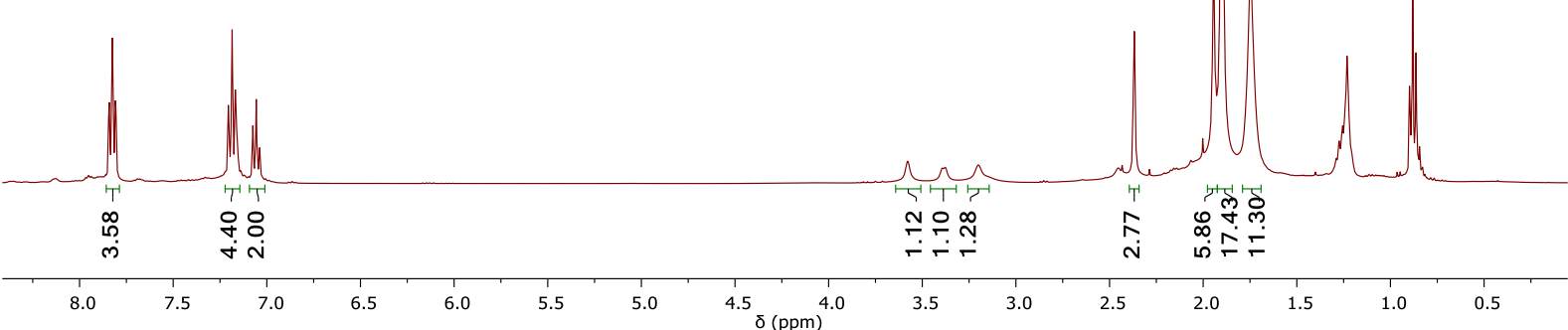

Figure S27. ${ }^{1} \mathrm{H}-\mathrm{NMR}\left(\mathrm{C}_{6} \mathrm{D}_{6}\right)$ of $\left[\mathrm{Ph}_{2} \mathrm{PCHC}(\mathrm{Me}) \mathrm{CH}_{2} \mathrm{Li}(\mathrm{PMDETA})\right] 3$ 


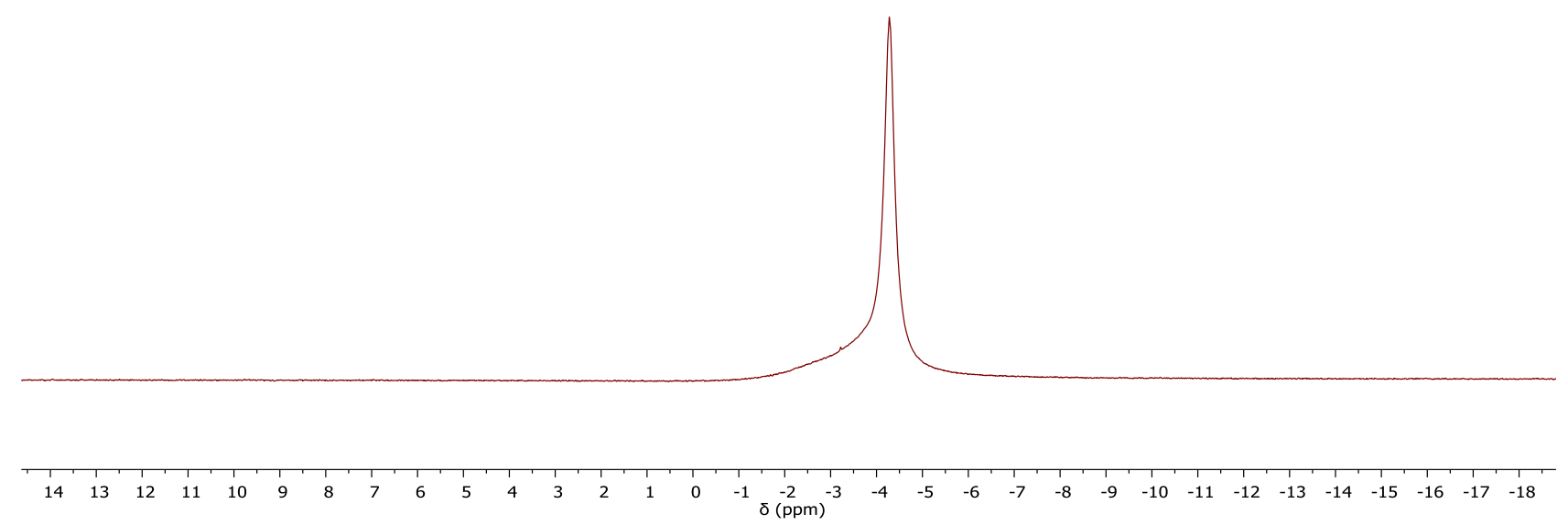

Figure S28. ${ }^{7} \mathrm{Li}-\mathrm{NMR}\left(\mathrm{C}_{6} \mathrm{D}_{6}\right)$ of $\left[\mathrm{Ph}_{2} \mathrm{PCHC}(\mathrm{Me}) \mathrm{CH}_{2} \mathrm{Li}(\mathrm{PMDETA})\right] 3$
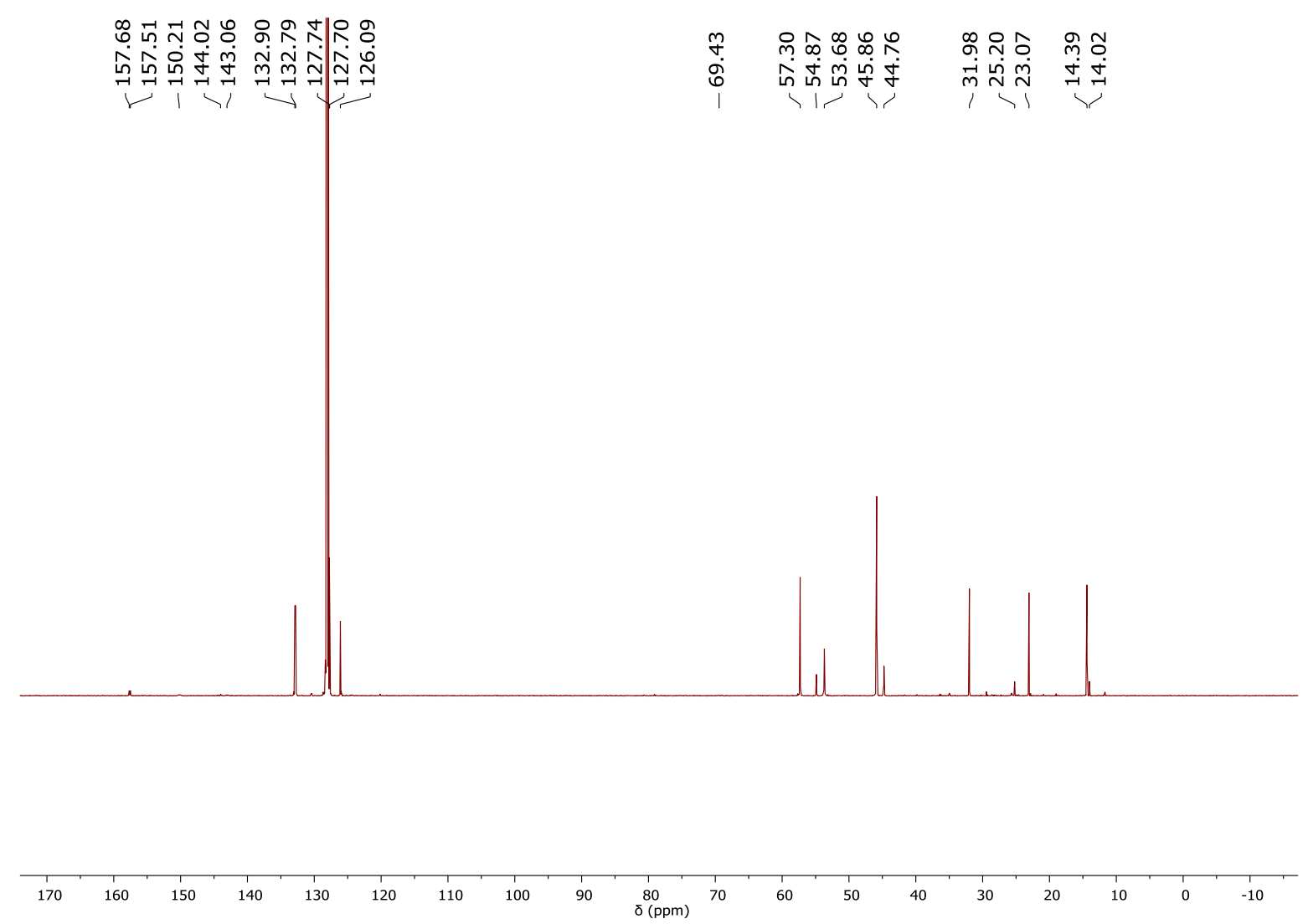

Figure S29. ${ }^{13} \mathrm{C}-\mathrm{NMR}\left(\mathrm{C}_{6} \mathrm{D}_{6}\right)$ of $\left[\mathrm{Ph}_{2} \mathrm{PCHC}(\mathrm{Me}) \mathrm{CH}_{2} \mathrm{Li}(\mathrm{PMDETA})\right] \mathbf{3}$ 


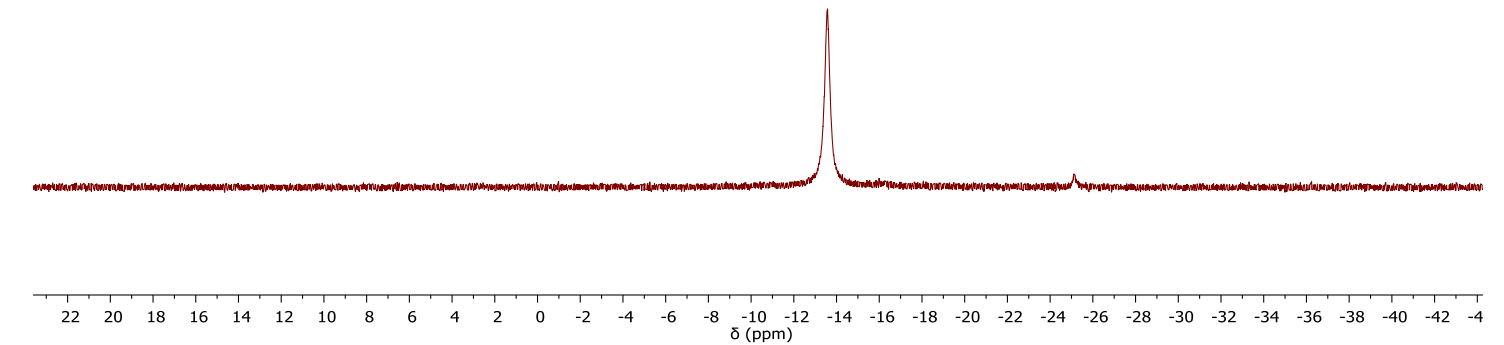

Figure S30. ${ }^{31} \mathrm{P}\{\mathrm{H}\}-\mathrm{NMR}\left(\mathrm{C}_{6} \mathrm{D}_{6}\right)$ of $\left[\mathrm{Ph}_{2} \mathrm{PCHC}(\mathrm{Me}) \mathrm{CH}_{2} \mathrm{Li}(\mathrm{PMDETA})\right] 3$

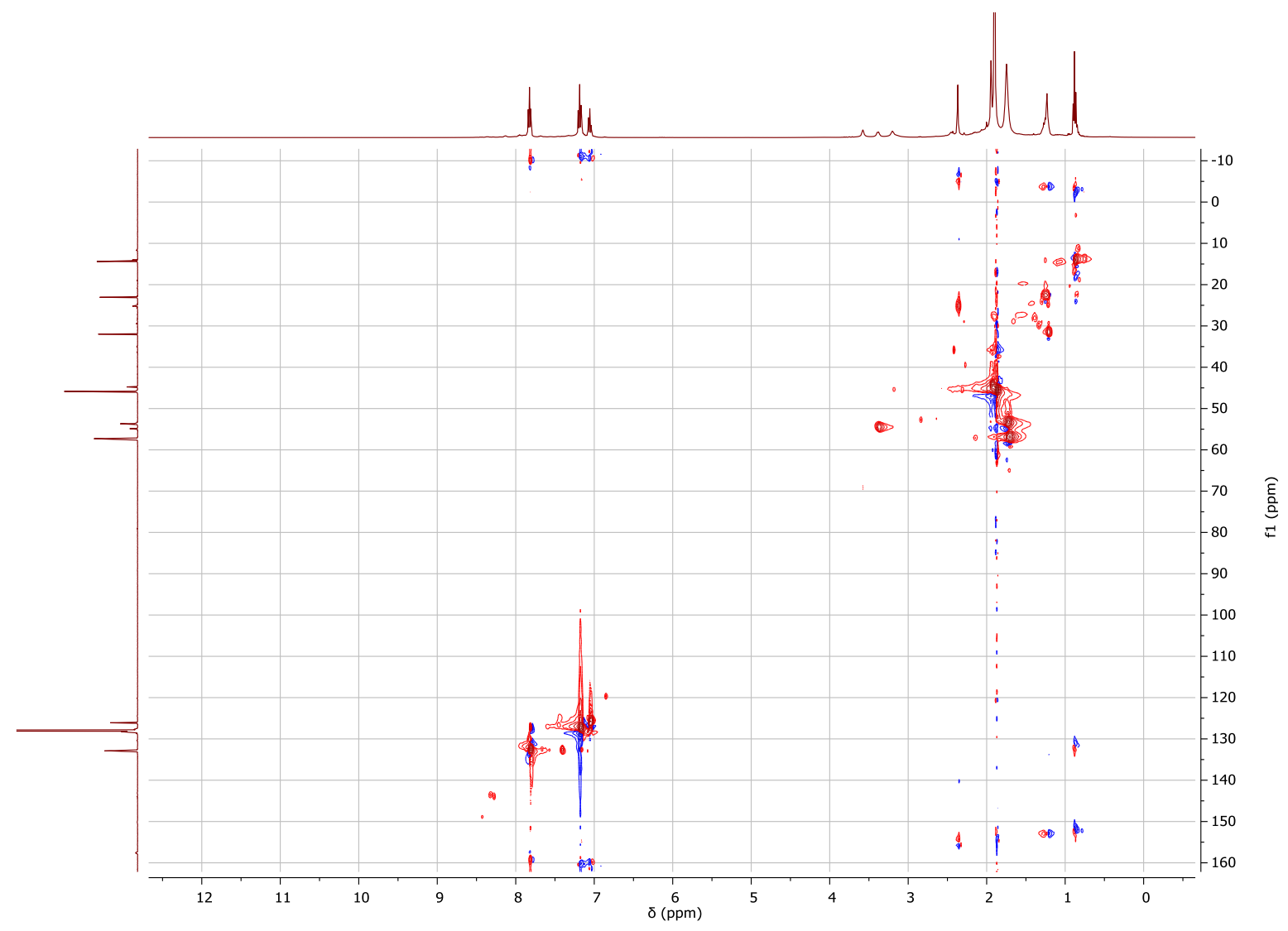

Figure S31. HMQC-NMR $\left(\mathrm{C}_{6} \mathrm{D}_{6}\right)$ of $\left[\mathrm{Ph}_{2} \mathrm{PCHC}(\mathrm{Me}) \mathrm{CH}_{2} \mathrm{Li}(\mathrm{PMDETA})\right] 3$ 


\section{NMR spectra of [PhP $\left.\left\{\mathrm{CHCHCH}_{2} \mathrm{Li}(\mathrm{TMEDA})\right\}_{2}\right]$ 4:}

${ }^{1} \mathrm{H}$ NMR $\left(\mathrm{C}_{6} \mathrm{D}_{6}, 400.20 \mathrm{MHz}\right): \delta 1.85\left(\mathrm{~s}, 8 \mathrm{H}, \mathrm{TMEDA}-\mathrm{CH}_{2}\right), 2.04\left(\mathrm{~s}, 24 \mathrm{H}, \mathrm{TMEDA}-\mathrm{CH}_{3}\right), 3.16\left(\mathrm{br}-\mathrm{m}, 4 \mathrm{H},=\mathrm{CH}_{2}\right), 3.62(\mathrm{br}-$ m, 2H, P-CH-), 6.95 (br-m, 2H, -CH=), 7.09 (m, 1H, $p-\mathrm{H}), 7.35$ (m, 2H, $m-\mathrm{H}), 8.08$ (m, 2H, $o-\mathrm{H})$.

${ }^{7} \mathrm{Li}$ NMR ( $\left.\mathrm{C}_{6} \mathrm{D}_{6}, 155.53 \mathrm{MHz}\right): \delta-5.14$.

${ }^{13} \mathrm{C}$ NMR ( $\left.\mathrm{C}_{6} \mathrm{D}_{6}, 100.6 \mathrm{MHz}\right): \delta 46.12$ (s, TMEDA-Me), 56.55 (s, TMEDA-CH2), 65.02 (br-m, allyl-C), 73.57 (br-m, allylC), $105.72(\mathrm{~m},=\mathrm{CH} 2), 124.65$ (br-s, $p-\mathrm{C}), 127.47\left(\mathrm{~d},{ }^{3} J_{\mathrm{C}-\mathrm{P}}=3.9 \mathrm{~Hz}, m-\mathrm{C}\right), 130.78$ (br-d, $\left.{ }^{2} J_{\mathrm{C}-\mathrm{P}}=12.9 \mathrm{~Hz}, o-\mathrm{C}\right), 140.02(\mathrm{br}-\mathrm{m}$, $-\mathrm{CH}=), 151.31$ (br-m, $i$-C).

${ }^{31} \mathrm{P}\left\{{ }^{1} \mathrm{H}\right\}$ NMR $\left(\mathrm{C}_{6} \mathrm{D}_{6}, 162.0 \mathrm{MHz}\right): \delta-35.2$.

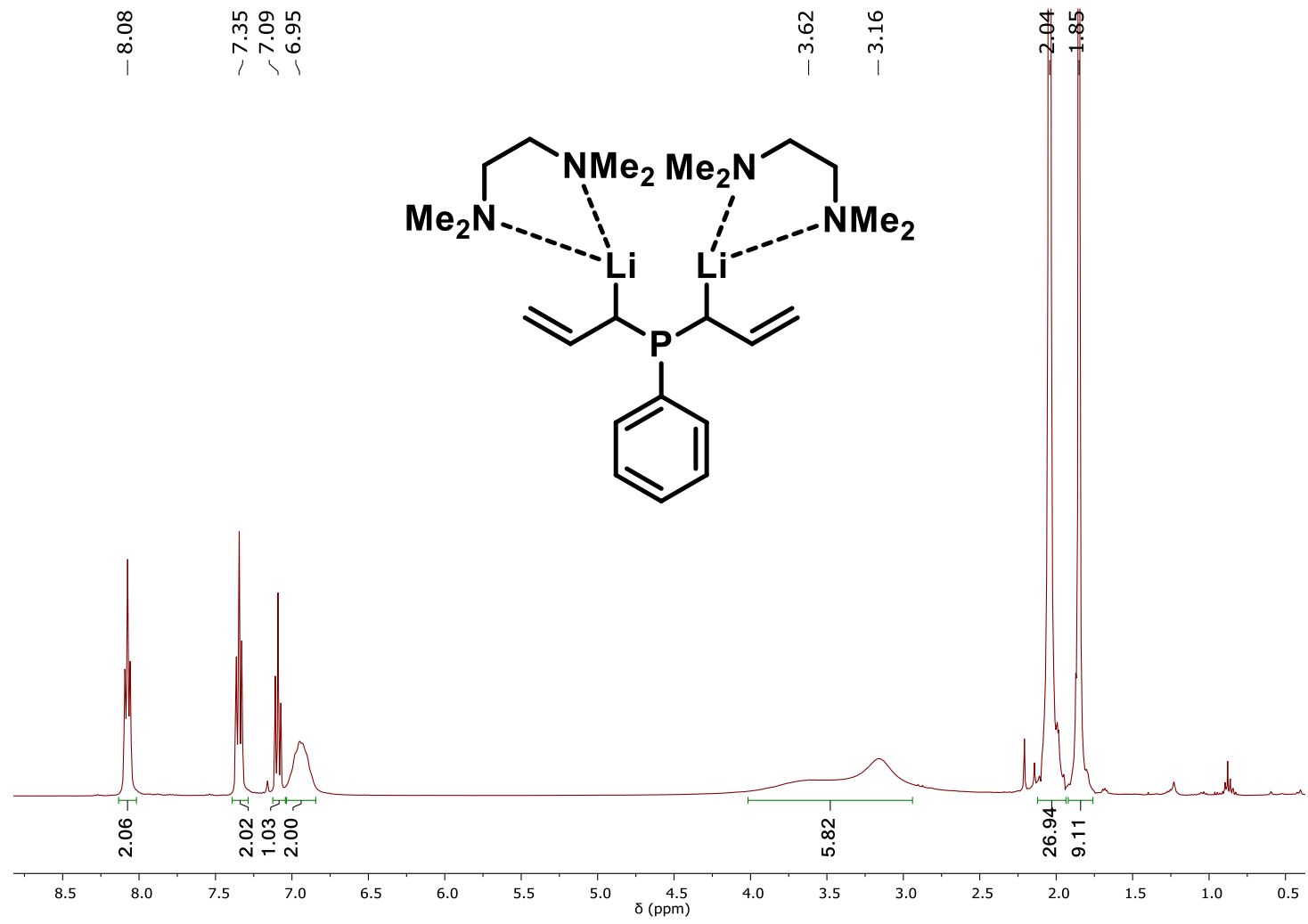

Figure S32. ${ }^{1} \mathrm{H}-\mathrm{NMR}\left(\mathrm{C}_{6} \mathrm{D}_{6}\right)$ of $\left[\mathrm{PhP}\left\{\mathrm{CHCHCH}_{2} \mathrm{Li}(\mathrm{TMEDA})\right\}_{2}\right] 4$ 


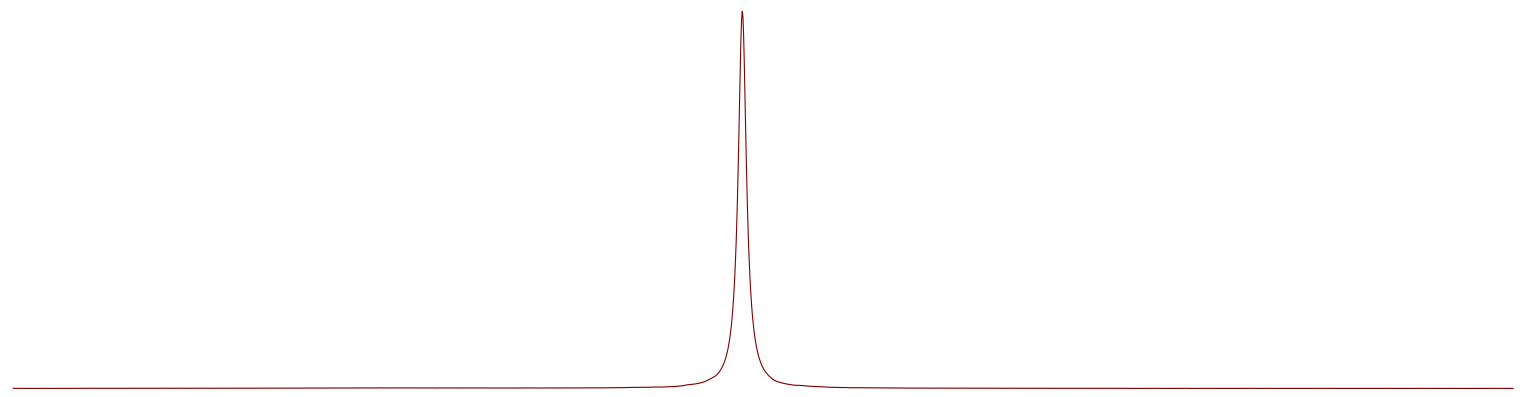

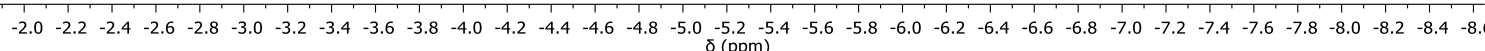

Figure S33. ${ }^{7} \mathrm{Li}-\mathrm{NMR}\left(\mathrm{C}_{6} \mathrm{D}_{6}\right)$ of $\left[\mathrm{PhP}\left\{\mathrm{CHCHCH}_{2} \mathrm{Li}(\mathrm{TMEDA})\right\}_{2}\right] 4$

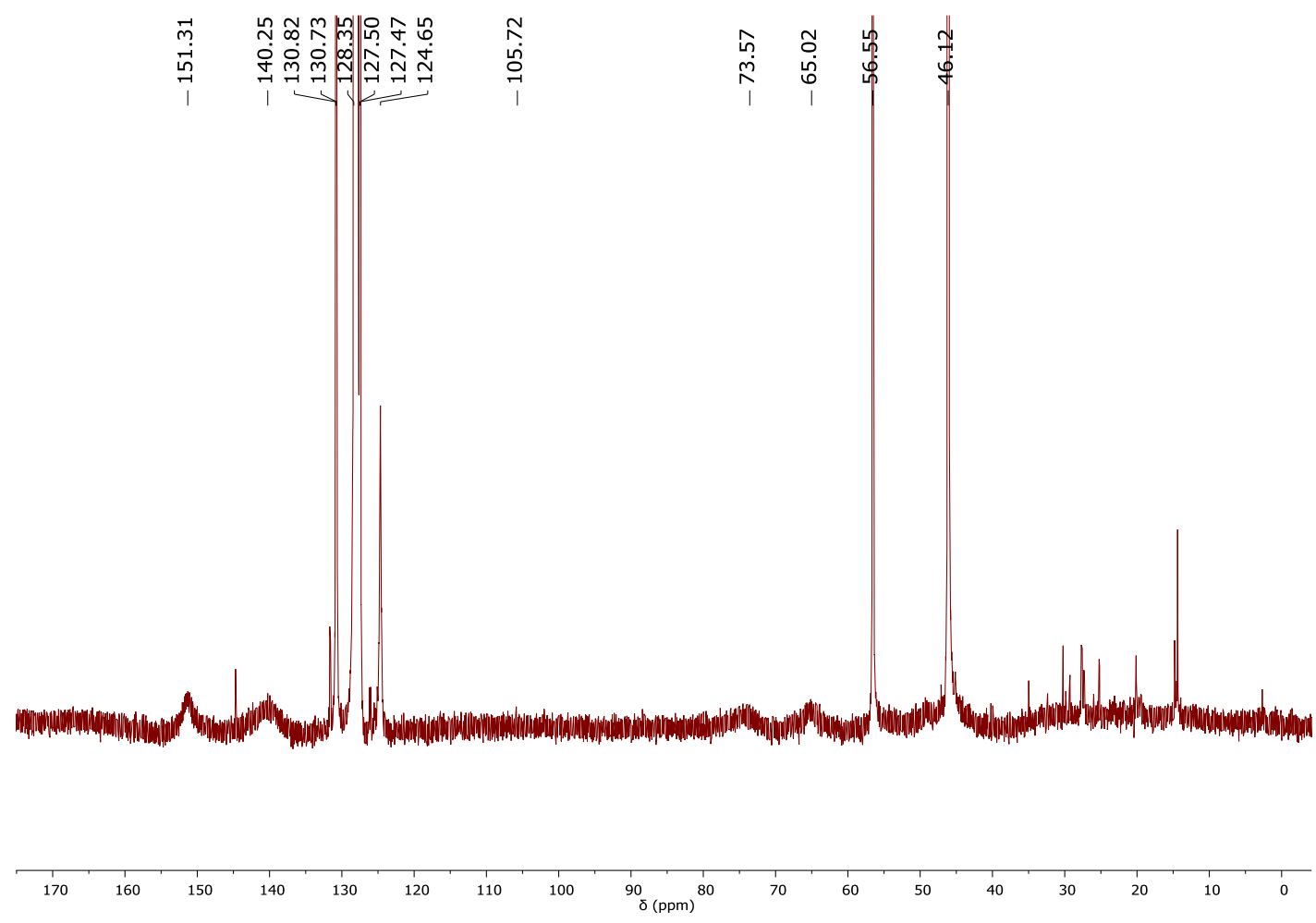

Figure S34. ${ }^{13} \mathrm{C}-\mathrm{NMR}\left(\mathrm{C}_{6} \mathrm{D}_{6}\right)$ of $\left[\mathrm{PhP}\left\{\mathrm{CHCHCH}_{2} \mathrm{Li}(\mathrm{TMEDA})\right\}_{2}\right] 4$ 


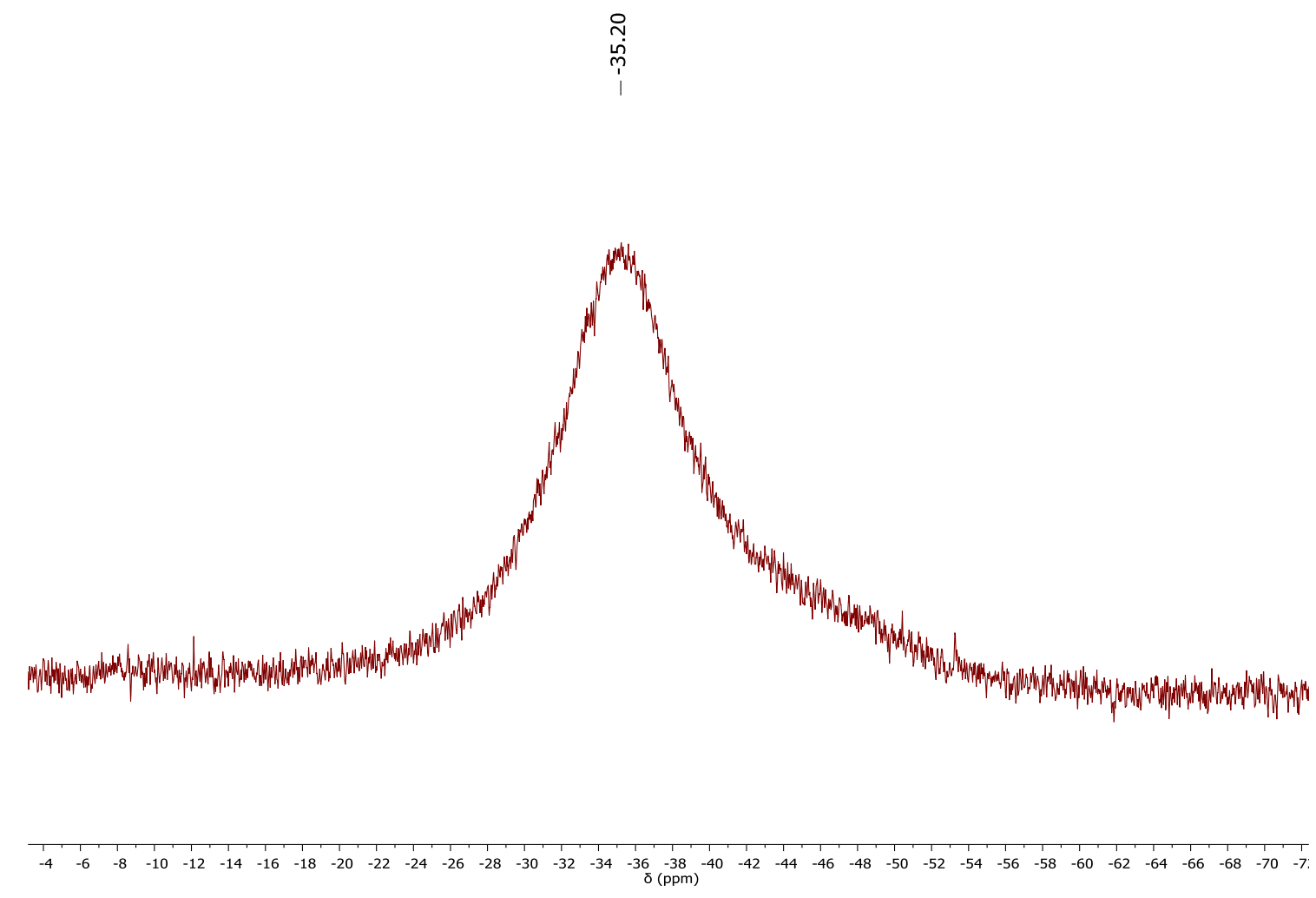

Figure S35. ${ }^{31} \mathrm{P}\{\mathrm{H}\}-\mathrm{NMR}\left(\mathrm{C}_{6} \mathrm{D}_{6}\right)$ of $\left[\mathrm{PhP}\left\{\mathrm{CHCHCH}_{2} \mathrm{Li}(\mathrm{TMEDA})\right\}_{2}\right] 4$ 


\section{NMR spectra of $\left[\left\{\mathrm{Ph}_{2} \mathrm{P}(\mathrm{O}) \mathrm{CHC}(\mathrm{Me}) \mathrm{CH}_{2} \mathrm{Li}(\mathrm{THF})\right\}_{2}\right] \mathbf{5}_{\text {: }}$}

${ }^{1} \mathrm{H}$ NMR $\left(100{ }^{\circ} \mathrm{C}, \mathrm{C}_{7} \mathrm{D}_{8}, 400.20 \mathrm{MHz}\right): \delta 1.46(\mathrm{~m}, 4 \mathrm{H}, \mathrm{THF}), 1.95$ (br-s, 3H, -C(Me)=), $2.72\left(\mathrm{dd},{ }^{3} J_{\mathrm{H}-\mathrm{P}}=24.7 \mathrm{~Hz},{ }^{4} J_{\mathrm{H}-\mathrm{H}}=1.5\right.$ $\mathrm{Hz}, 1 \mathrm{H}, \mathrm{P}-\mathrm{CH}-), 3.52(\mathrm{~m}, 4 \mathrm{H}, \mathrm{THF}), 3.57(\mathrm{~s}, 1 \mathrm{H},=\mathrm{CHH}), 3.96(\mathrm{~s}, 1 \mathrm{H},=\mathrm{CHH}), 7.06(\mathrm{~m}, 6 \mathrm{H}, m$ and $p-\mathrm{H}), 7.80(\mathrm{~m}, 4 \mathrm{H}, o-\mathrm{H})$.

${ }^{1} \mathrm{H}$ NMR $\left(25^{\circ} \mathrm{C}\right.$, THF-d8, $\left.400.20 \mathrm{MHz}\right): \delta 1.67$ (br-s, 3H, -C(Me)=), $1.68(\mathrm{~m}, 4 \mathrm{H}, \mathrm{THF}), 2.33\left(\mathrm{~d},{ }^{3} J_{\mathrm{H}-\mathrm{P}}=24.6 \mathrm{~Hz}, 1 \mathrm{H}, \mathrm{P}-\mathrm{CH}-\right.$ ), $3.14(\mathrm{~s}, 1 \mathrm{H},=\mathrm{CHH}), 3.39\left(\mathrm{~d},{ }^{2} J_{\mathrm{H}-\mathrm{H}}=24.6 \mathrm{~Hz}, 1 \mathrm{H},=\mathrm{CHH}\right), 3.54(\mathrm{~m}, 4 \mathrm{H}, \mathrm{THF}), 7.15(\mathrm{~m}, 6 \mathrm{H}, m$ and $p-\mathrm{H}), 7.81(\mathrm{~m}, 4 \mathrm{H}, o-$ $\mathrm{H})$.

${ }^{7} \mathrm{Li}$ NMR $\left(25^{\circ} \mathrm{C}, \mathrm{C}_{7} \mathrm{D}_{8}, 155.53 \mathrm{MHz}\right): \delta-3.82$.

${ }^{7} \mathrm{Li} \mathrm{NMR}\left(25^{\circ} \mathrm{C}, \mathrm{THF}-\mathrm{d}_{8}, 155.53 \mathrm{MHz}\right): \delta-4.04,-4.50$.

${ }^{13} \mathrm{C}$ NMR $\left(25{ }^{\circ} \mathrm{C}, \mathrm{C}_{7} \mathrm{D}_{8}, 100.6 \mathrm{MHz}\right): \delta 26.03(\mathrm{~s}, \mathrm{THF}), 28.13\left(\mathrm{~d},{ }^{2} J_{\mathrm{C}-\mathrm{P}}=17.6 \mathrm{~Hz},-\mathrm{C}(\mathrm{Me})=\right), 45.94\left(\mathrm{~d},{ }^{1} J_{\mathrm{C}-\mathrm{P}}=133.2 \mathrm{~Hz}, \mathrm{P}-\right.$ CH-), 68.37 (s, THF), $81.62\left(\mathrm{~m},=\mathrm{CH}_{2}\right), 128.75$ (m, $p$-C), 130.75 (s, $\left.m-\mathrm{C}\right), 132.27$ (s, $\left.o-\mathrm{C}\right), 151.78$ (m, $i$-C).

${ }^{13} \mathrm{C}$ NMR $\left(25{ }^{\circ} \mathrm{C}\right.$, THF-d8, $\left.100.6 \mathrm{MHz}\right): \delta 26.54$ (s, THF), $27.74\left(\mathrm{~d},{ }^{2} J_{\mathrm{C}-\mathrm{P}}=16.8 \mathrm{~Hz},-\mathrm{C}(\mathrm{Me})=\right), 48.15\left(\mathrm{~d},{ }^{1} J_{\mathrm{C}-\mathrm{P}}=144.0 \mathrm{~Hz}, \mathrm{P}-\right.$ $\mathrm{CH}-), 68.39$ (s, THF), 82.31 (br s, $\left.=\mathrm{CH}_{2}\right), 128.02\left(\mathrm{~d},{ }^{3} J_{\mathrm{C}-\mathrm{P}}=11.0 \mathrm{~Hz}, m-\mathrm{C}\right), 129.40(\mathrm{~s}, p-\mathrm{C}), 133.00\left(\mathrm{~d},{ }^{2} J_{\mathrm{C}-\mathrm{P}}=8.9 \mathrm{~Hz}, o-\mathrm{C}\right)$, $142.30\left(\mathrm{~d},{ }^{1} J_{\mathrm{C}-\mathrm{P}}=105.9 \mathrm{~Hz}, i-\mathrm{C}\right), 149.00$ (br s, $\left.-\mathrm{C}(\mathrm{Me})=\right)$.

${ }^{31} \mathrm{P}\left\{{ }^{1} \mathrm{H}\right\} \operatorname{NMR}\left(25{ }^{\circ} \mathrm{C}, \mathrm{C}_{6} \mathrm{D}_{6}, 162.0 \mathrm{MHz}\right): \delta 36.2,38.2$.

${ }^{31} \mathrm{P}\left\{{ }^{1} \mathrm{H}\right\} \operatorname{NMR}\left(25^{\circ} \mathrm{C}, \mathrm{C}_{7} \mathrm{D} 8,162.0 \mathrm{MHz}\right): \delta 36.0,37.7$.

${ }^{31} \mathrm{P}\left\{{ }^{1} \mathrm{H}\right\}$ NMR $\left(100{ }^{\circ} \mathrm{C}, \mathrm{C}_{7} \mathrm{D}_{8}, 162.0 \mathrm{MHz}\right): \delta 37.0$.

${ }^{31} \mathrm{P}\left\{{ }^{1} \mathrm{H}\right\}$ NMR $\left(25^{\circ} \mathrm{C}, \mathrm{THF}-\mathrm{d} 8,162.0 \mathrm{MHz}\right): \delta 26.5,30.7,30.9$.

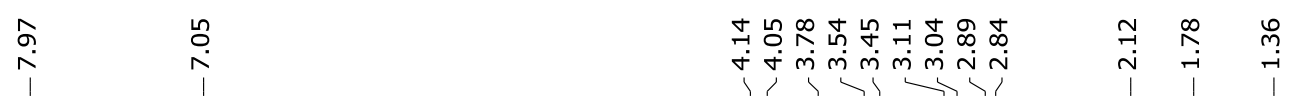

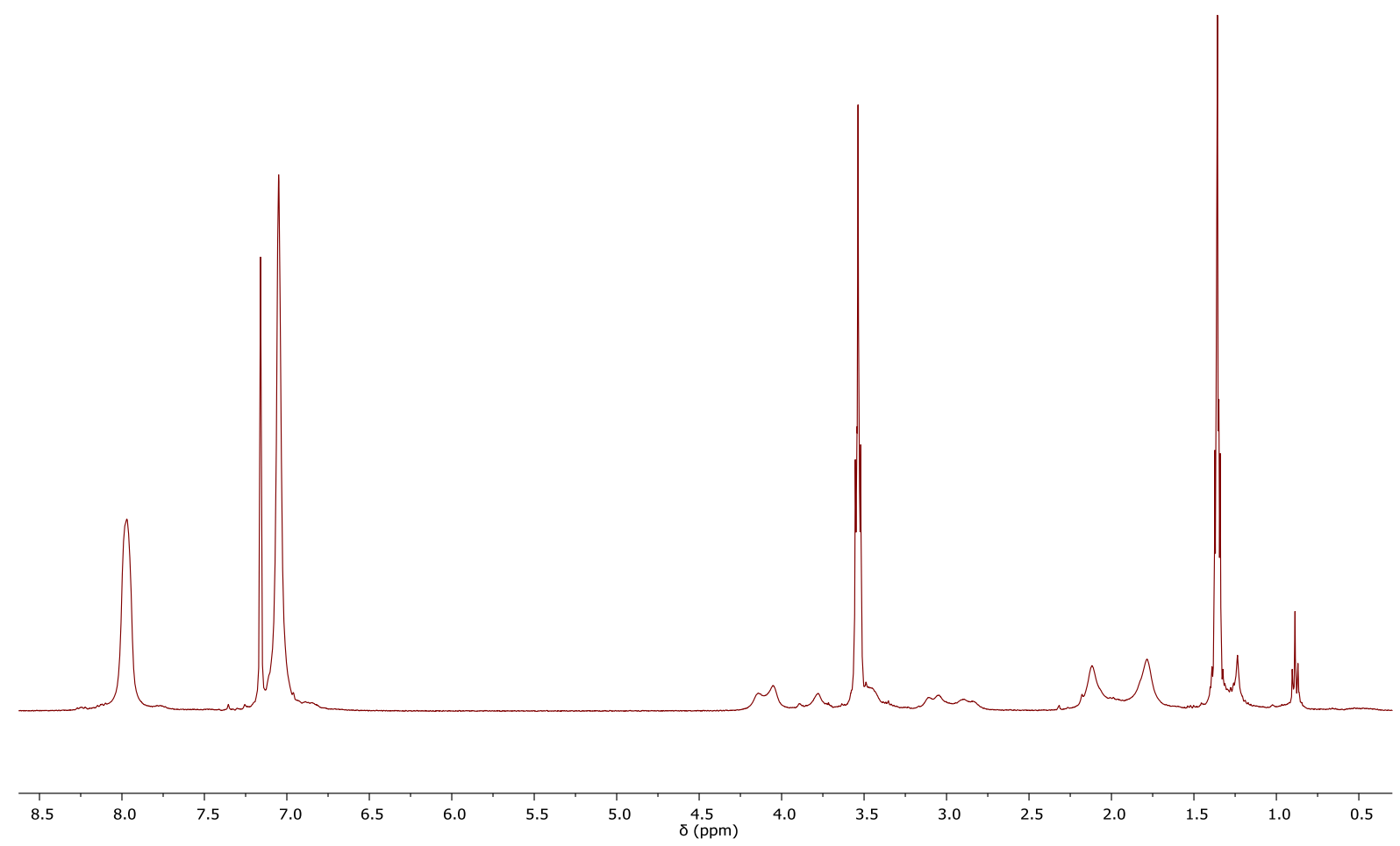

Figure S36. ${ }^{1} \mathrm{H}-\mathrm{NMR}\left(\mathrm{C}_{6} \mathrm{D}_{6}, 25^{\circ} \mathrm{C}\right)$ of $\left[\left\{\mathrm{Ph}_{2} \mathrm{P}(\mathrm{O}) \mathrm{CHC}(\mathrm{Me}) \mathrm{CH}_{2} \mathrm{Li}(\mathrm{THF})\right\}_{2}\right] 5$ 


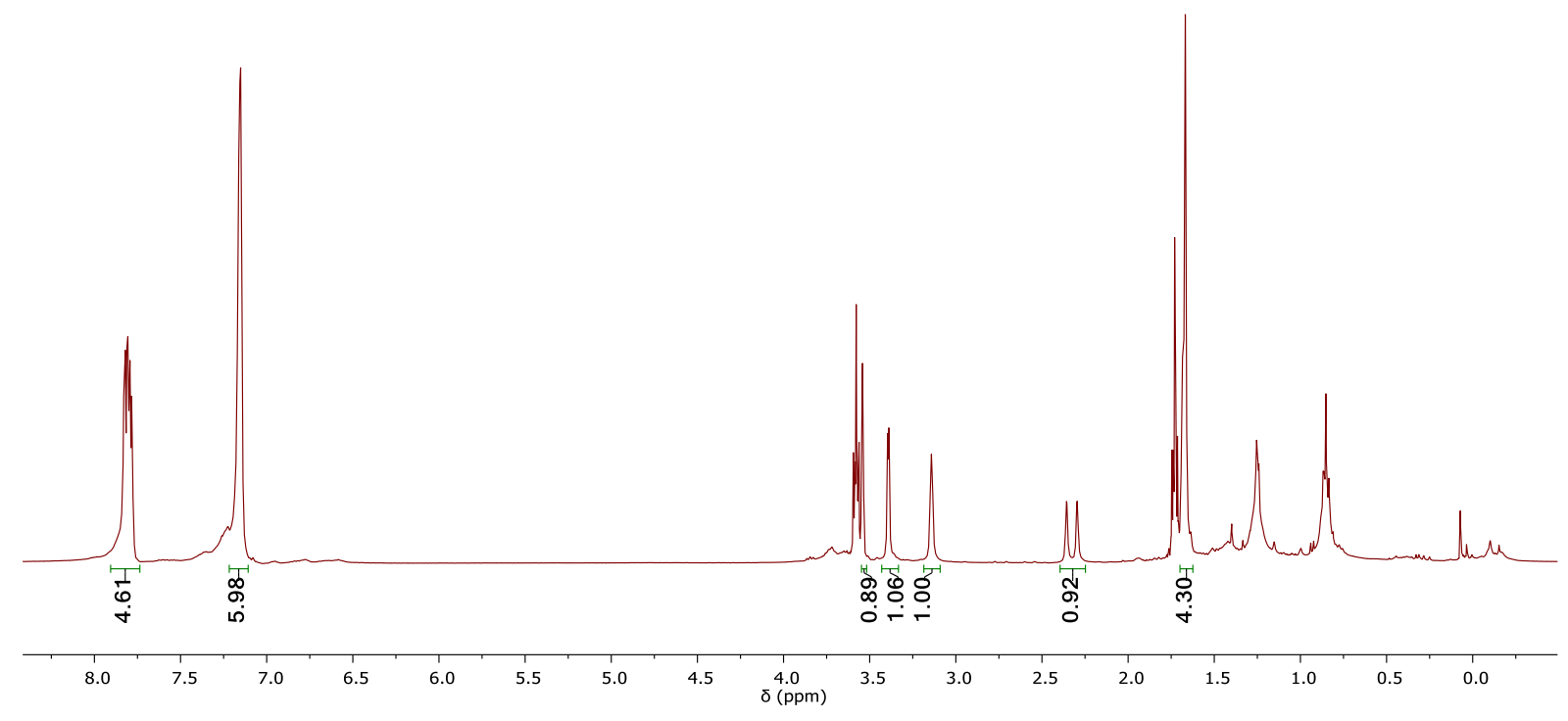

Figure S37. ${ }^{1} \mathrm{H}-\mathrm{NMR}\left(\mathrm{THF}-\mathrm{d}_{8}, 25^{\circ} \mathrm{C}\right)$ of $\left[\left\{\mathrm{Ph}_{2} \mathrm{P}(\mathrm{O}) \mathrm{CHC}(\mathrm{Me}) \mathrm{CH}_{2} \mathrm{Li}(\mathrm{THF})\right\}_{2}\right] 5$

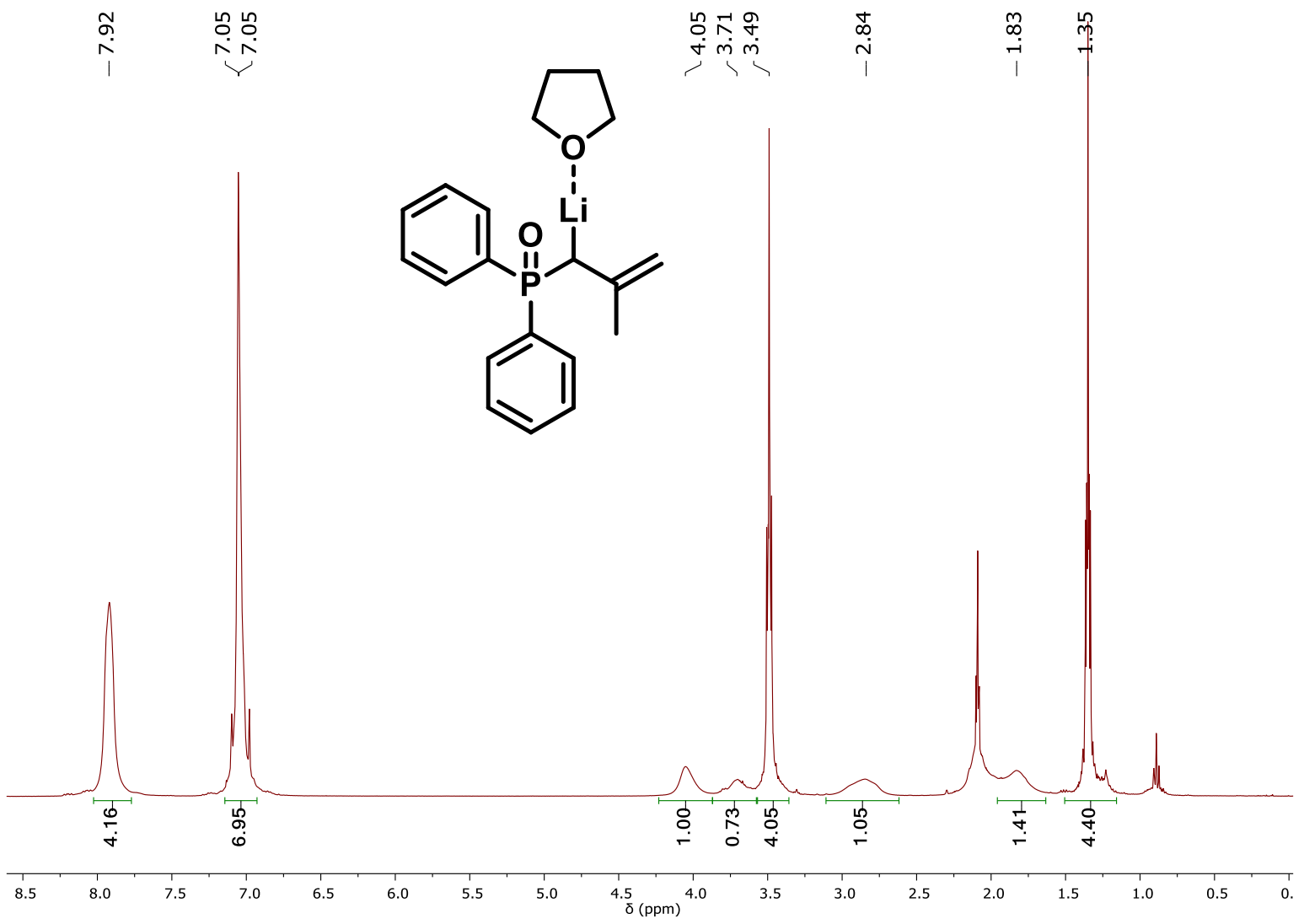

Figure S38. ${ }^{1} \mathrm{H}-\mathrm{NMR}$ (Toluene $-\mathrm{d}_{8}, 25^{\circ} \mathrm{C}$ ) of $\left[\left\{\mathrm{Ph}_{2} \mathrm{P}(\mathrm{O}) \mathrm{CHC}(\mathrm{Me}) \mathrm{CH}_{2} \mathrm{Li}(\mathrm{THF})\right\}_{2}\right] 5$ 


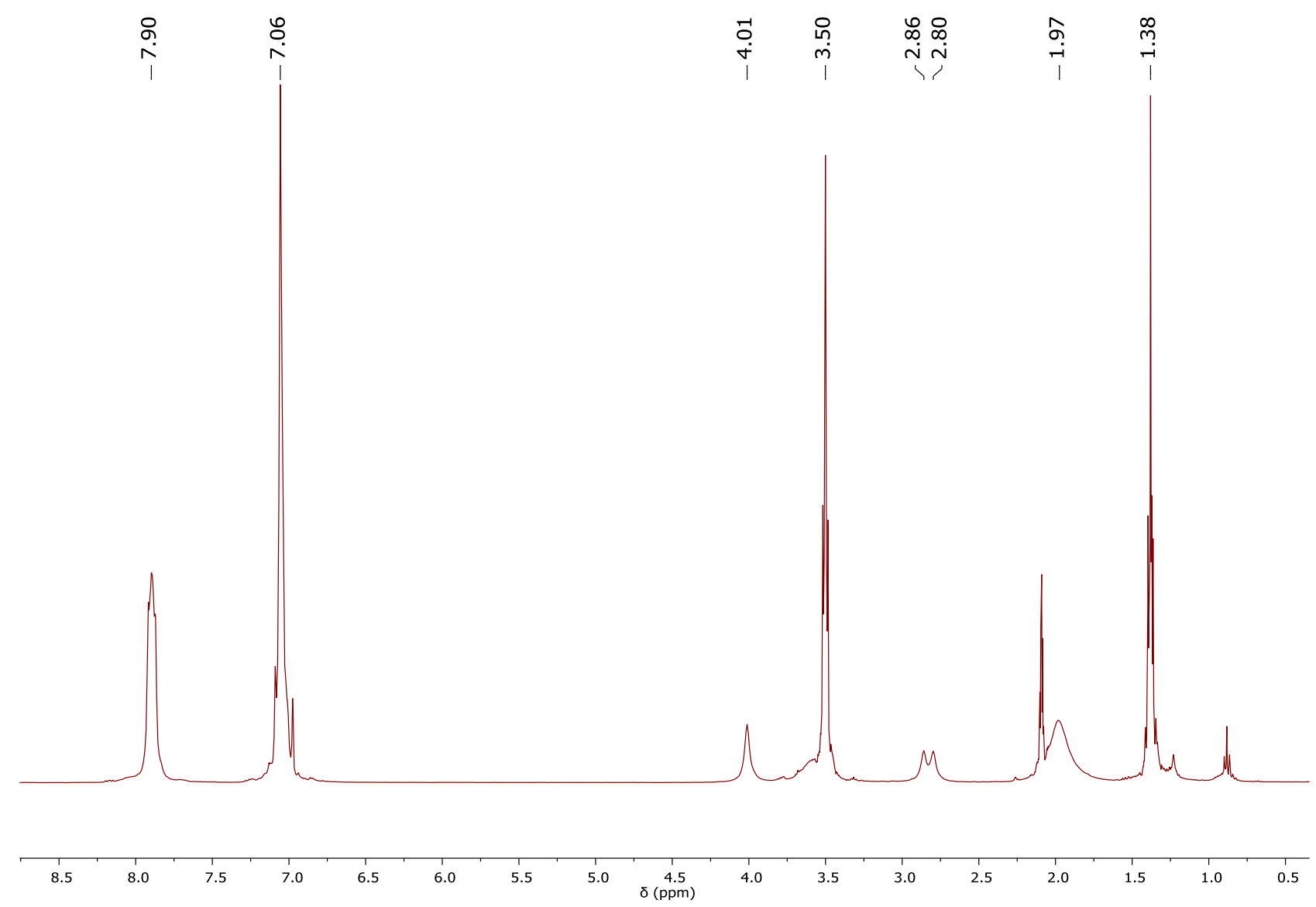

Figure S39. ${ }^{1} \mathrm{H}-\mathrm{NMR}$ (Toluene $-\mathrm{d}_{8}, 40{ }^{\circ} \mathrm{C}$ ) of $\left[\left\{\mathrm{Ph}_{2} \mathrm{P}(\mathrm{O}) \mathrm{CHC}(\mathrm{Me}) \mathrm{CH}_{2} \mathrm{Li}(\mathrm{THF})\right\}_{2}\right] 5$

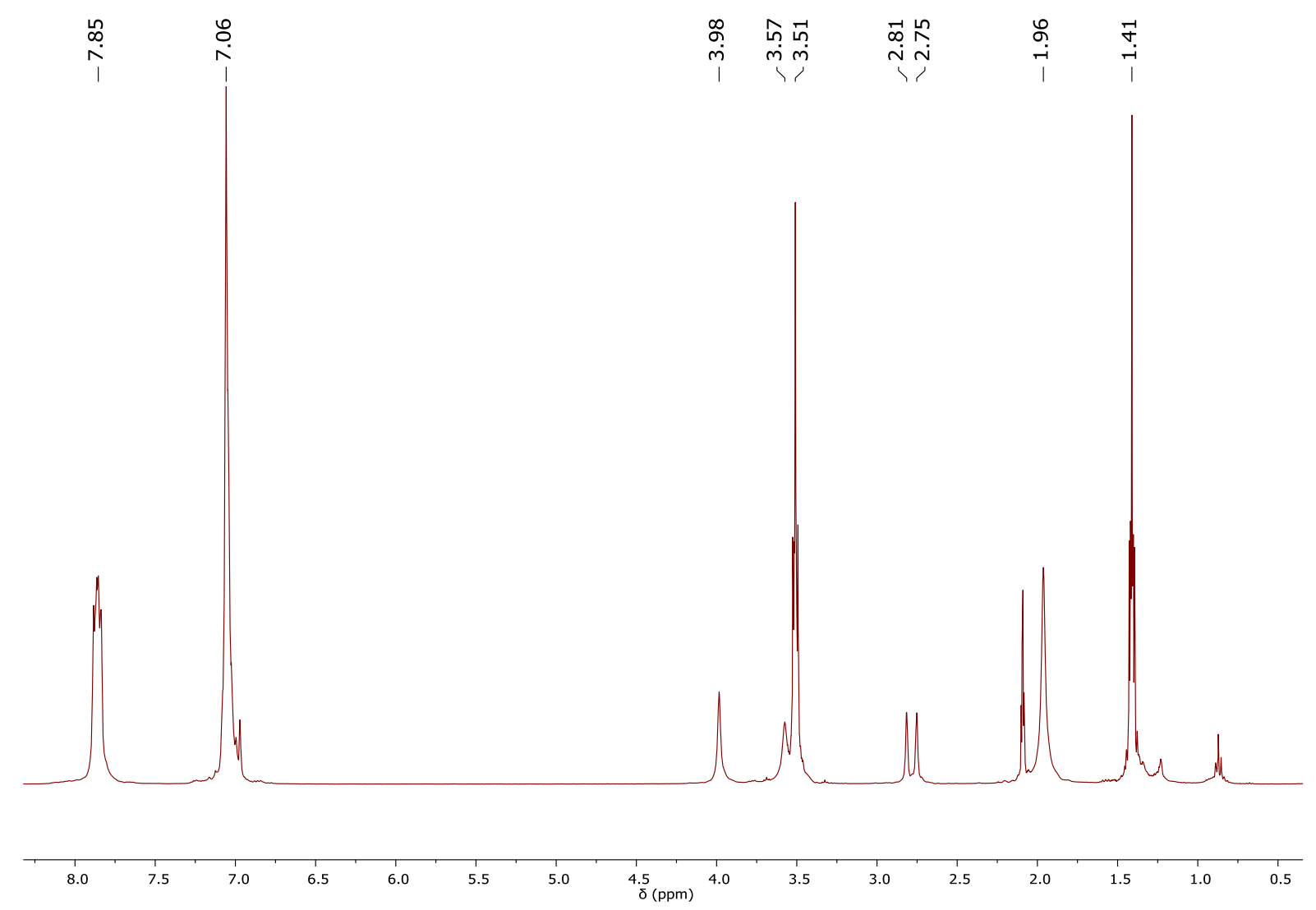

Figure S40. ${ }^{1} \mathrm{H}-\mathrm{NMR}$ (Toluene $-\mathrm{d}_{8}, 60{ }^{\circ} \mathrm{C}$ ) of $\left[\left\{\mathrm{Ph}_{2} \mathrm{P}(\mathrm{O}) \mathrm{CHC}(\mathrm{Me}) \mathrm{CH}_{2} \mathrm{Li}(\mathrm{THF})\right\}_{2}\right] 5$ 


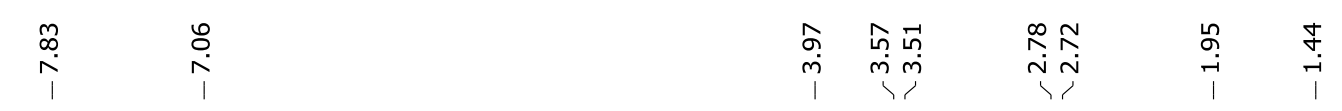

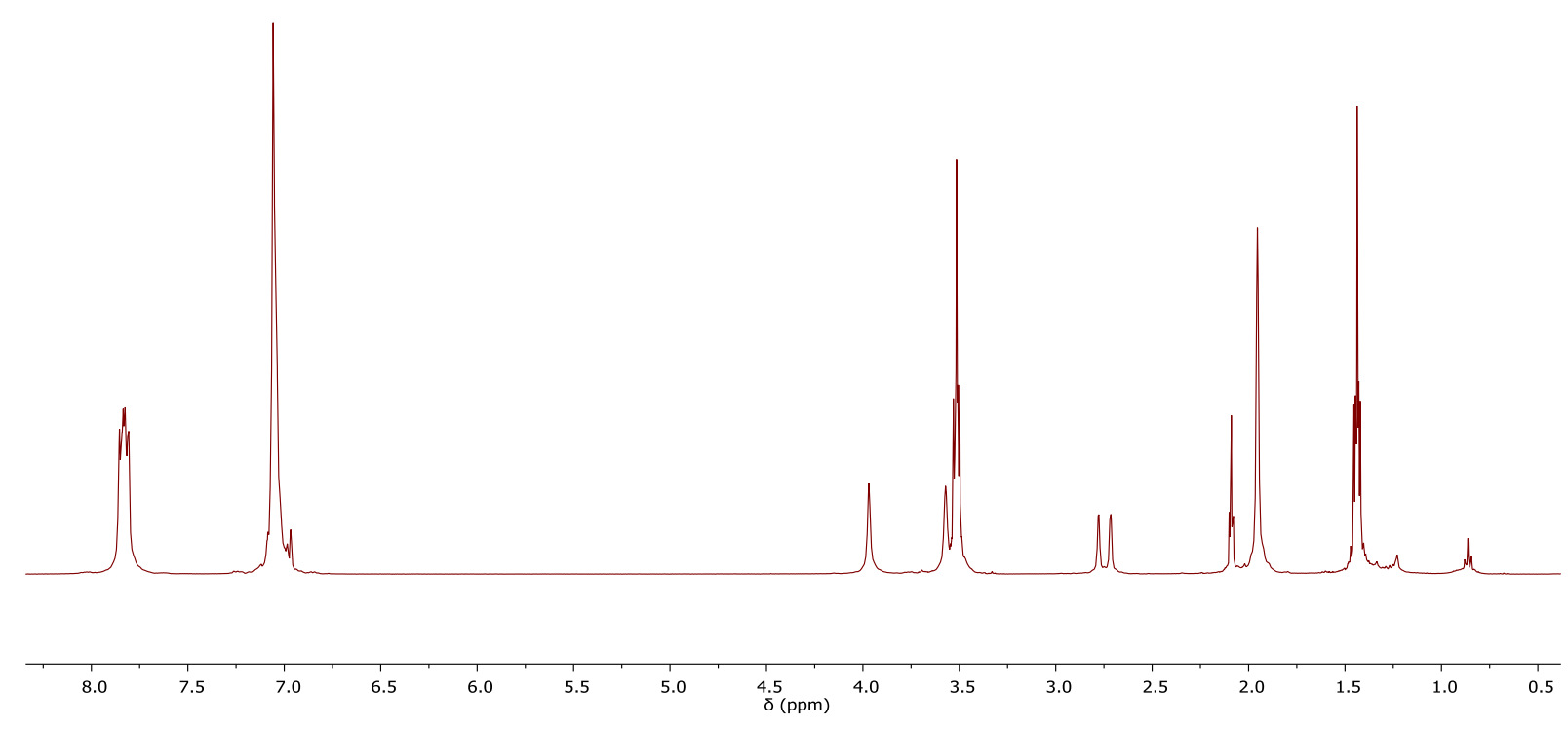

Figure S41. ${ }^{1} \mathrm{H}-\mathrm{NMR}$ (Toluene $-\mathrm{d}_{8}, 80{ }^{\circ} \mathrm{C}$ ) of $\left[\left\{\mathrm{Ph}_{2} \mathrm{P}(\mathrm{O}) \mathrm{CHC}(\mathrm{Me}) \mathrm{CH}_{2} \mathrm{Li}(\mathrm{THF})\right\}_{2}\right] 5$

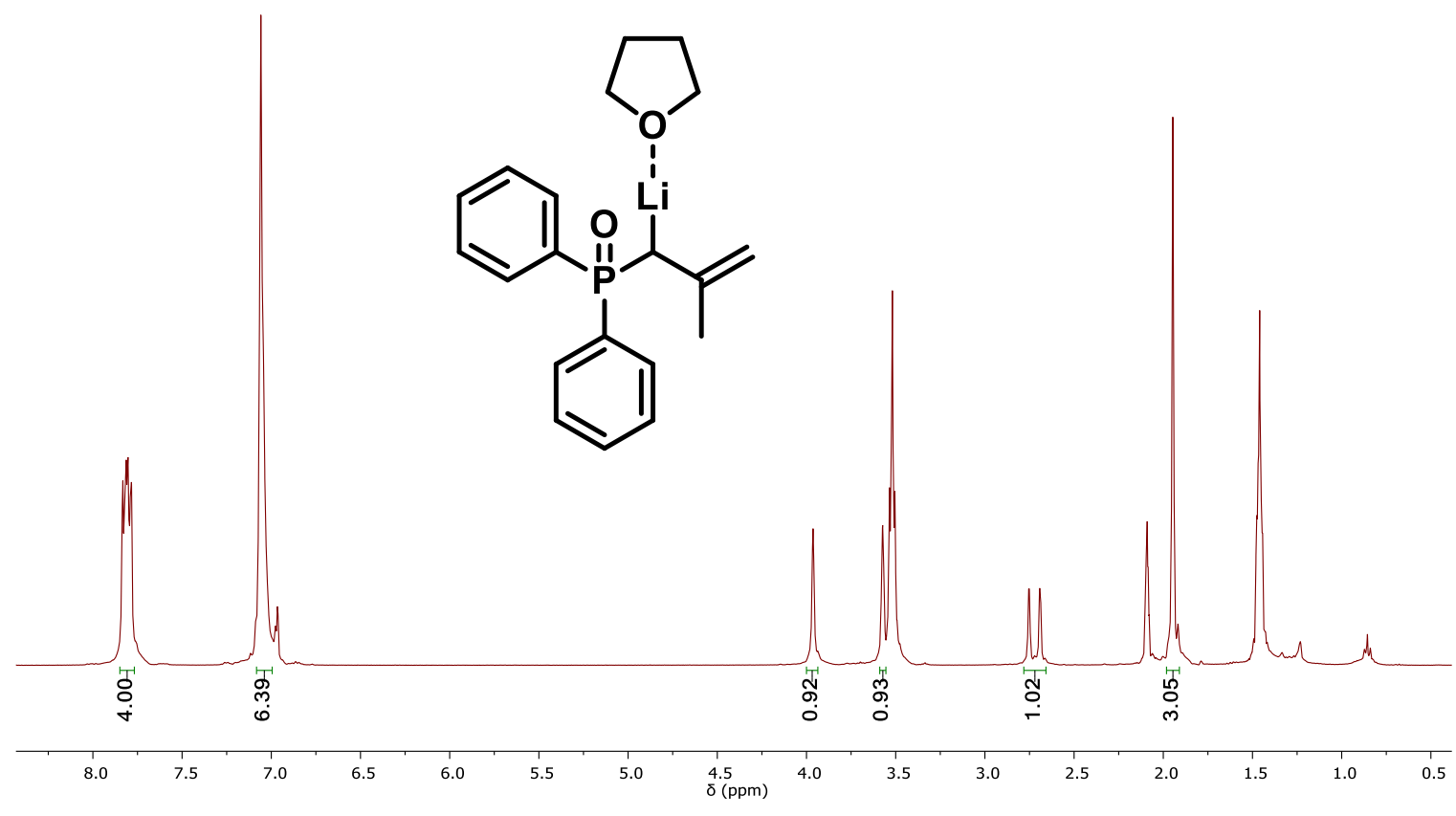

Figure S42. ${ }^{1} \mathrm{H}-\mathrm{NMR}$ (toluene $-\mathrm{d}_{8}, 100{ }^{\circ} \mathrm{C}$ ) of $\left[\left\{\mathrm{Ph}_{2} \mathrm{P}(\mathrm{O}) \mathrm{CHC}(\mathrm{Me}) \mathrm{CH}_{2} \mathrm{Li}(\mathrm{THF})\right\}_{2}\right] 5$ 


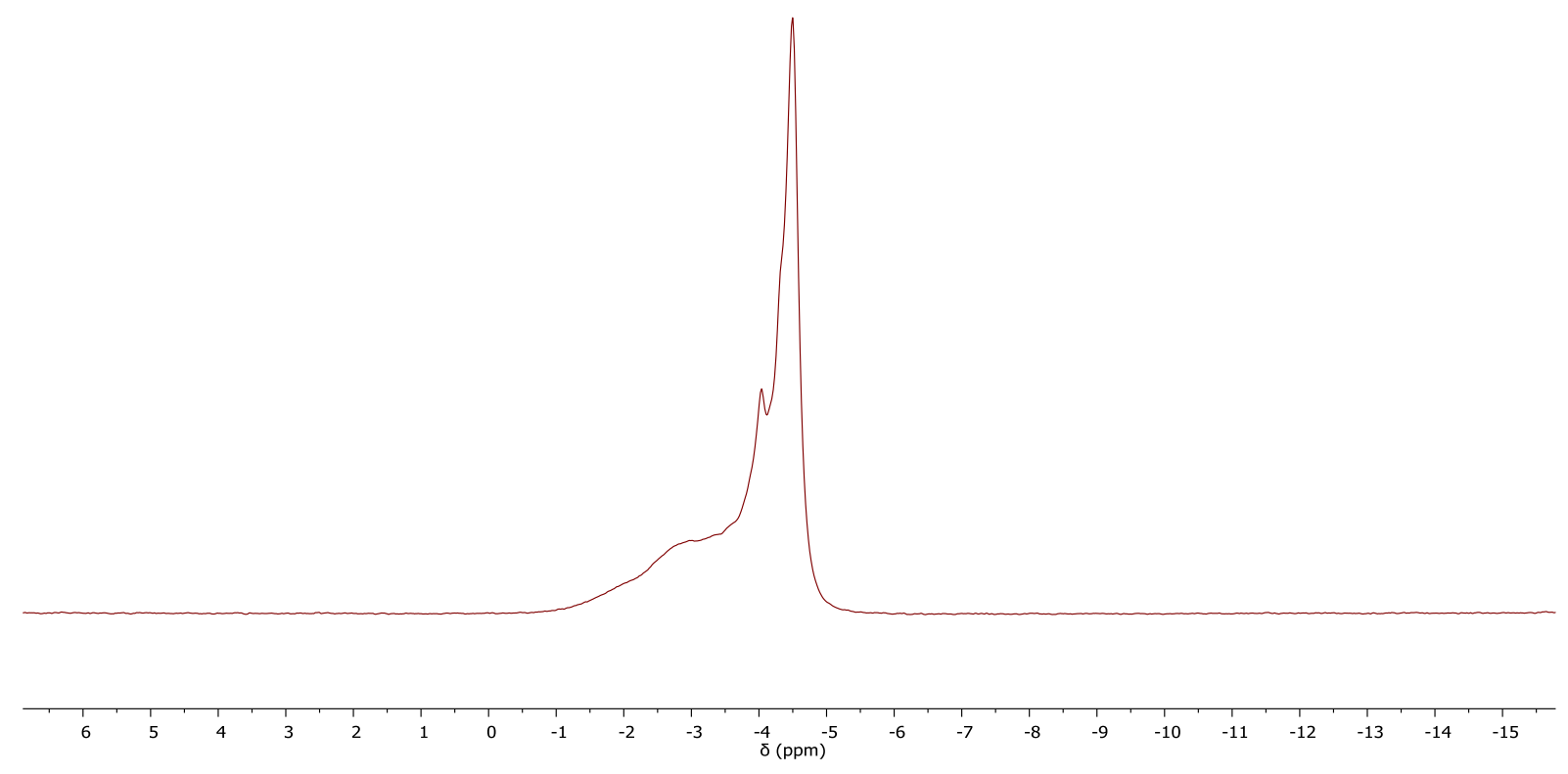

Figure S43. ${ }^{7} \mathrm{Li}-\mathrm{NMR}\left(\mathrm{THF}-\mathrm{d}_{8}, 2{ }^{\circ} \mathrm{C}\right.$ ) of $\left[\left\{\mathrm{Ph}_{2} \mathrm{P}(\mathrm{O}) \mathrm{CHC}(\mathrm{Me}) \mathrm{CH}_{2} \mathrm{Li}(\mathrm{THF})\right\}_{2}\right] 5$ 象 $\stackrel{\infty}{\dddot{i}}$

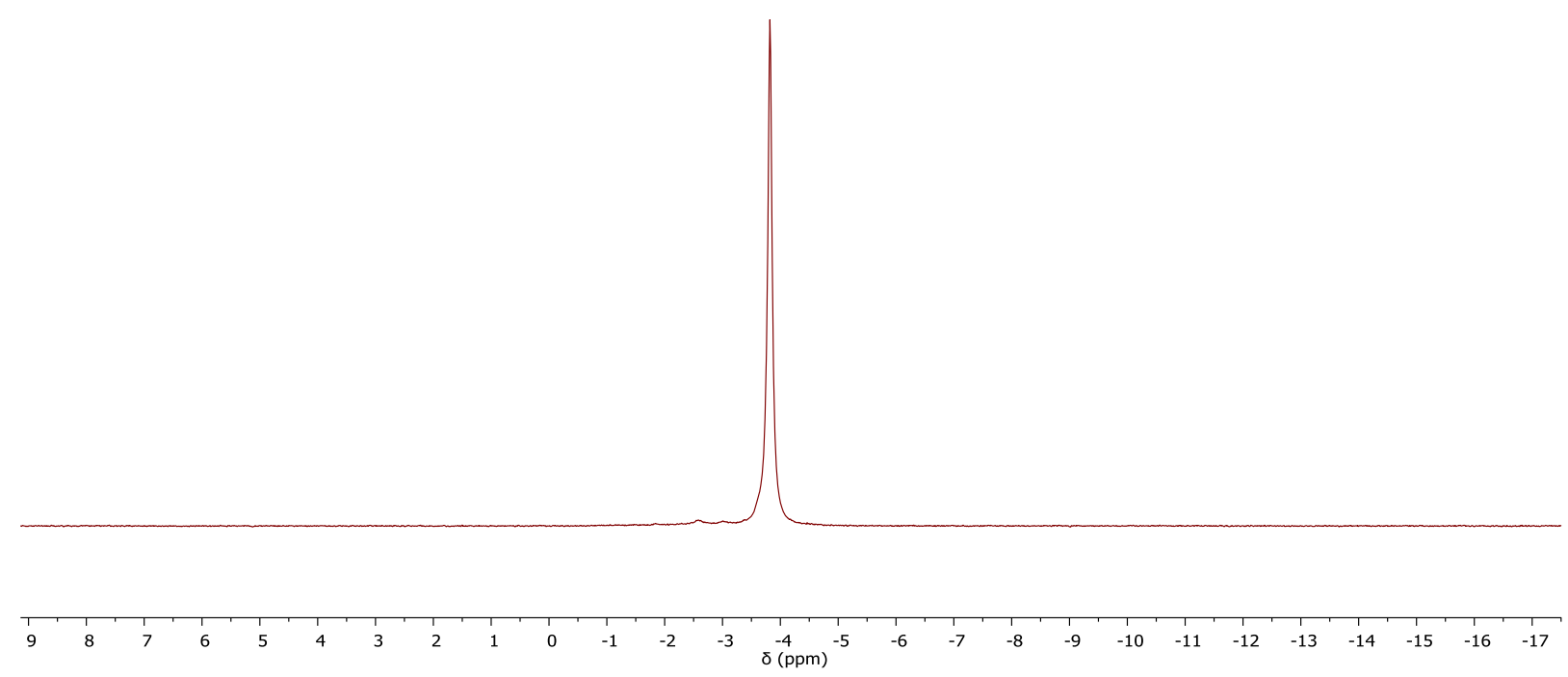

Figure S44. ${ }^{7} \mathrm{Li}-\mathrm{NMR}$ (toluene $-\mathrm{d}_{8}, 25^{\circ} \mathrm{C}$ ) of $\left[\left\{\mathrm{Ph}_{2} \mathrm{P}(\mathrm{O}) \mathrm{CHC}(\mathrm{Me}) \mathrm{CH}_{2} \mathrm{Li}(\mathrm{THF})\right\}_{2}\right] 5$ 


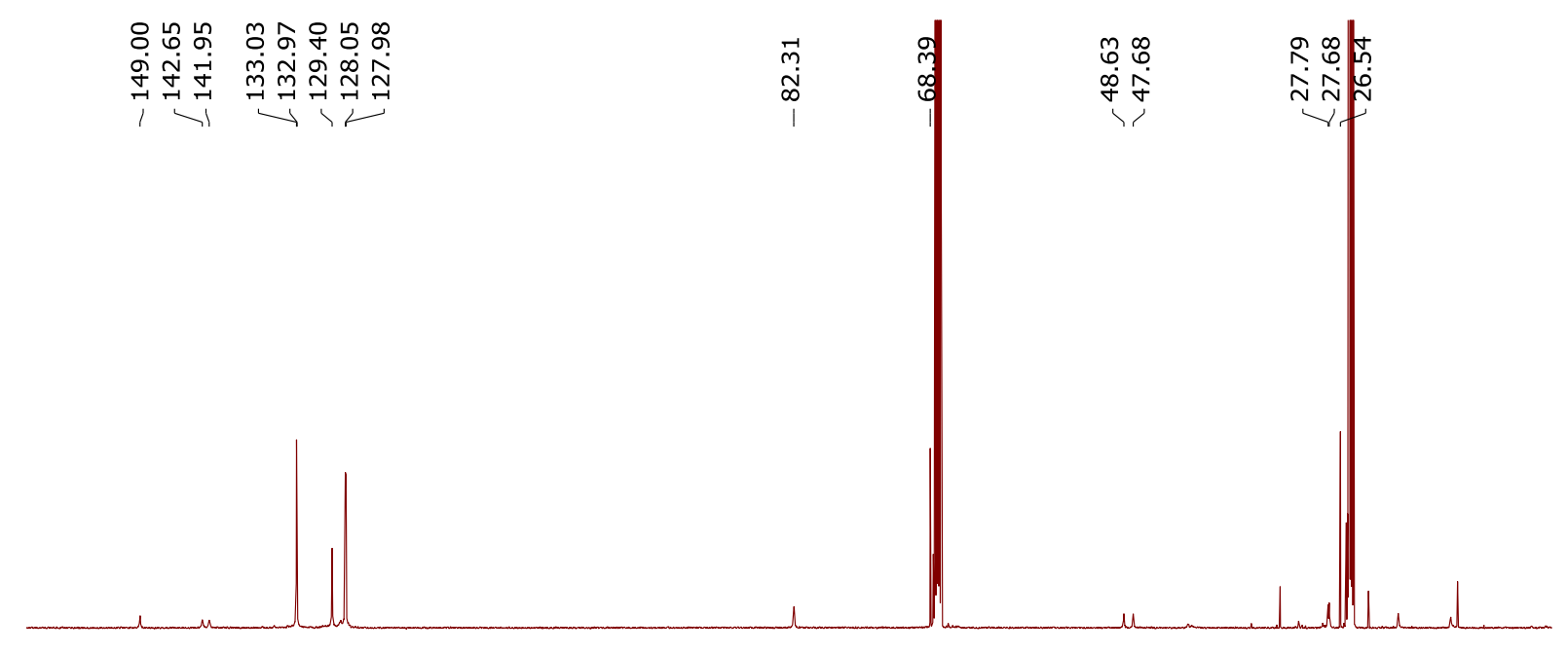

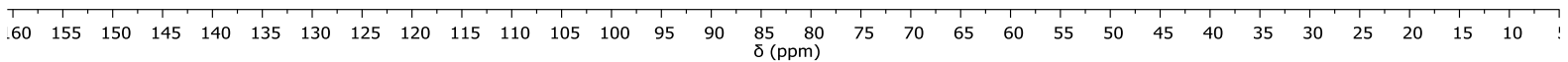

Figure S45. ${ }^{13} \mathrm{C}-\mathrm{NMR}\left(\mathrm{THF}-\mathrm{d}_{8}, 25{ }^{\circ} \mathrm{C}\right)$ of $\left[\left\{\mathrm{Ph}_{2} \mathrm{P}(\mathrm{O}) \mathrm{CHC}(\mathrm{Me}) \mathrm{CH}_{2} \mathrm{Li}(\mathrm{THF})\right\}_{2}\right] 5$

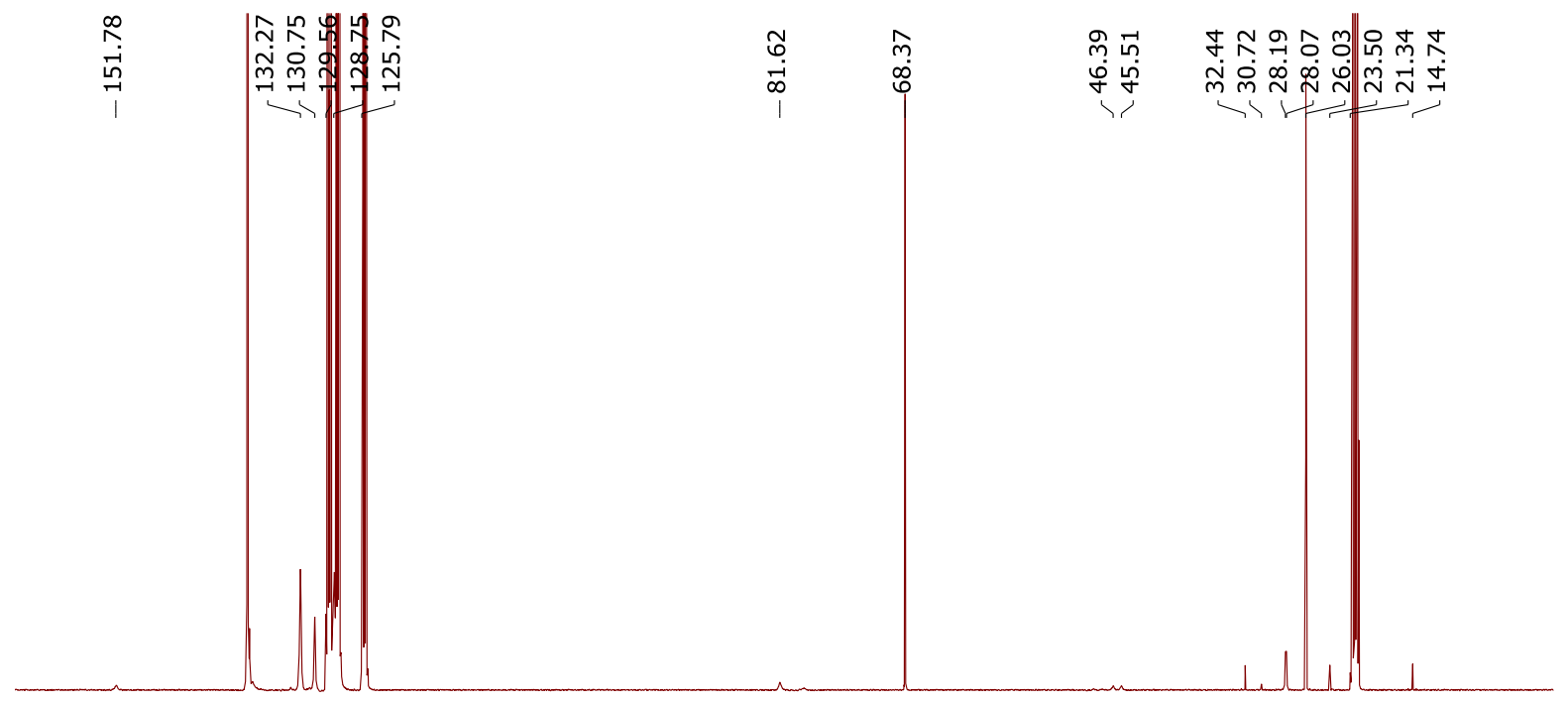

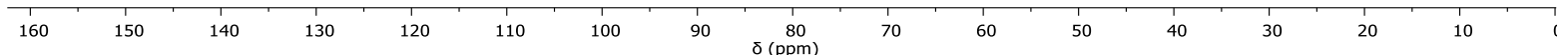

Figure S46. ${ }^{13} \mathrm{C}-\mathrm{NMR}$ (toluene $-\mathrm{d}_{8}, 25^{\circ} \mathrm{C}$ ) of $\left[\left\{\mathrm{Ph}_{2} \mathrm{P}(\mathrm{O}) \mathrm{CHC}(\mathrm{Me}) \mathrm{CH}_{2} \mathrm{Li}(\mathrm{THF})\right\}_{2}\right] 5$ 

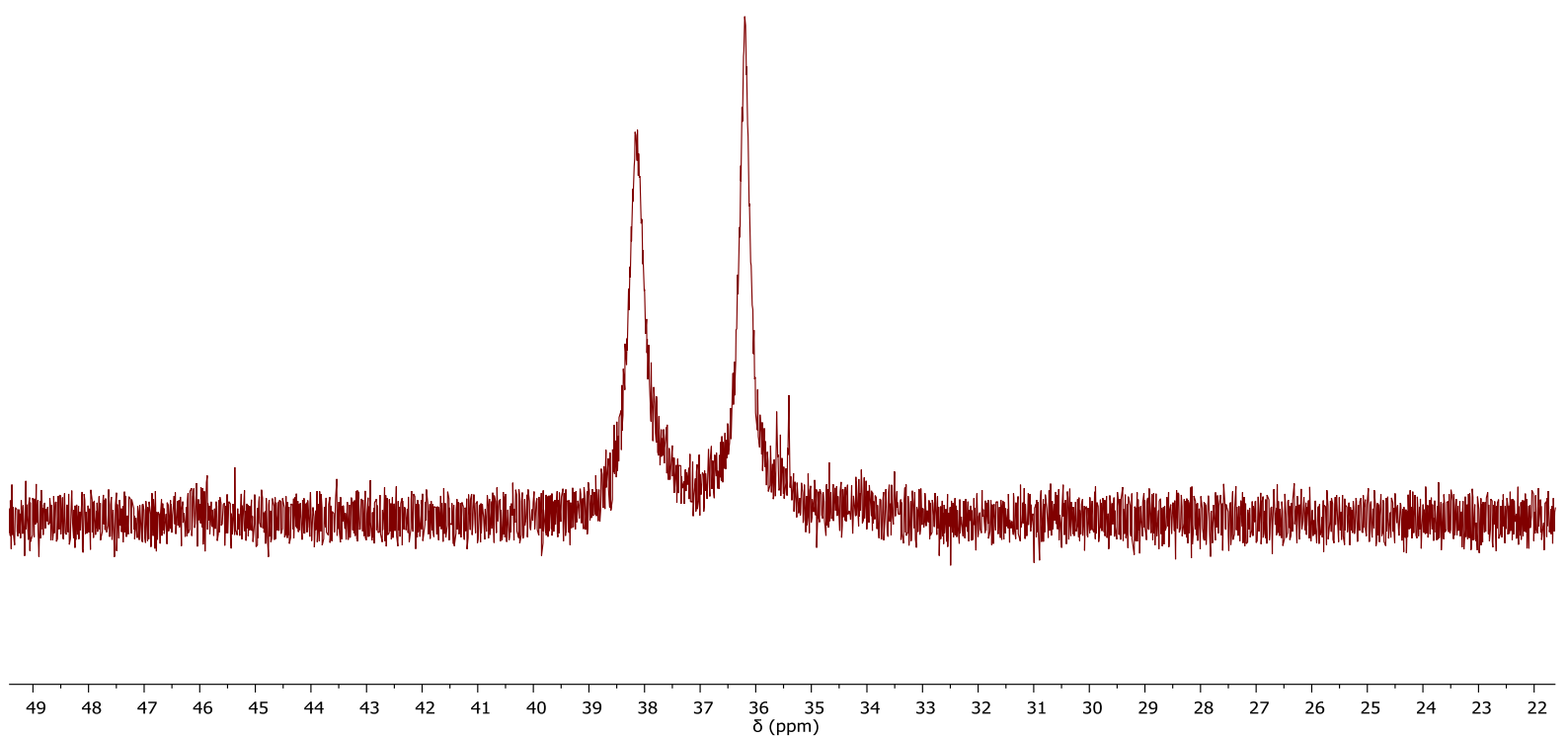

Figure S47. ${ }^{31} \mathrm{P}\{\mathrm{H}\}-\mathrm{NMR}\left(\mathrm{C}_{6} \mathrm{D}_{6}, 25^{\circ} \mathrm{C}\right)$ of $\left[\left\{\mathrm{Ph}_{2} \mathrm{P}(\mathrm{O}) \mathrm{CHC}(\mathrm{Me}) \mathrm{CH}_{2} \mathrm{Li}(\mathrm{THF})\right\}_{2}\right] \mathbf{5}$

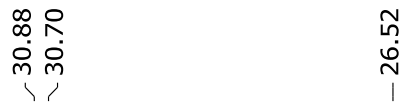

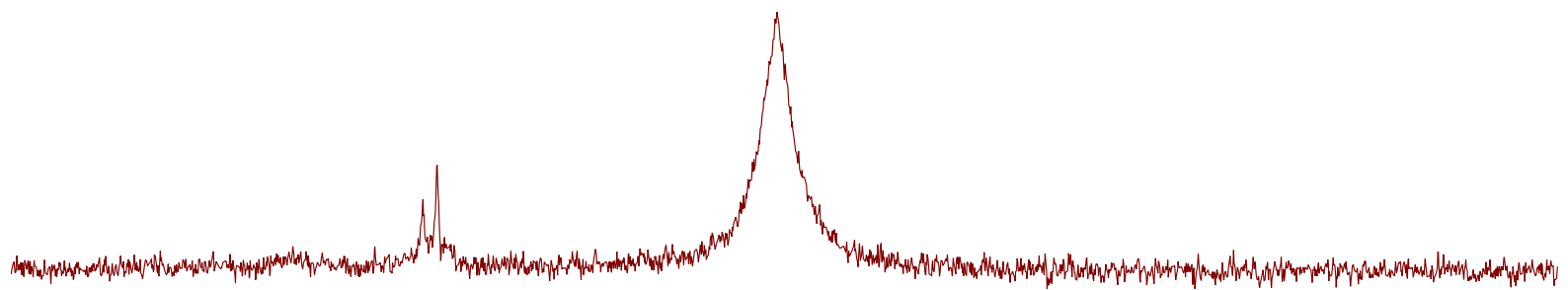

\begin{tabular}{llllllllllllllllllll}
1 & 1 \\
\hline 3 & 34 & 33 & 32 & 31 & 30 & 29 & 28 & 27 & 26 & 25 & 24 & 23 & 22 & 21 & 20 & 19 & 18 & 1
\end{tabular}

Figure S48. ${ }^{31} \mathrm{P}\{\mathrm{H}\}-\mathrm{NMR}\left(\mathrm{THF}-\mathrm{d}_{8}, 25^{\circ} \mathrm{C}\right)$ of $\left[\left\{\mathrm{Ph}_{2} \mathrm{P}(\mathrm{O}) \mathrm{CHC}(\mathrm{Me}) \mathrm{CH}_{2} \mathrm{Li}(\mathrm{THF})\right\}_{2}\right] 5$ 


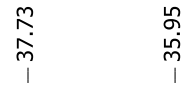

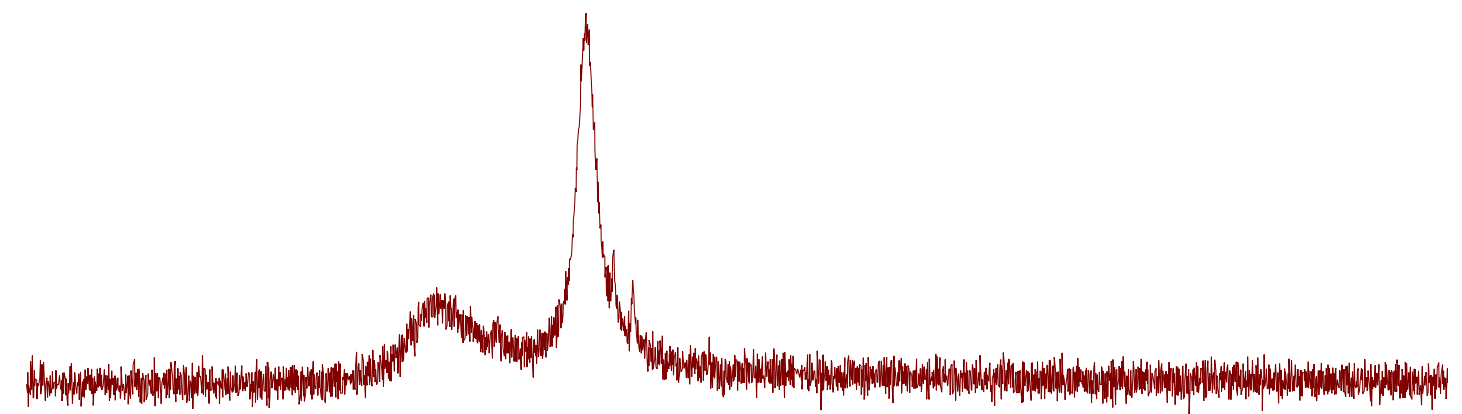

\begin{tabular}{lllllllllllllllll}
1 & 1 & 1 & 1 & 1 & 1 & 1 & 1 & 1 \\
\hline & 41 & 40 & 39 & 38 & 37 & 36 & 35 & $\begin{array}{c}34 \\
(\mathrm{ppm})\end{array}$ & 33 & 32 & 31 & 30 & 29 & 28 & 27 & 26
\end{tabular}

Figure S49. ${ }^{31} \mathrm{P}\{\mathrm{H}\}-\mathrm{NMR}$ (toluene- $\mathrm{d}_{8}, 25^{\circ} \mathrm{C}$ ) of $\left[\left\{\mathrm{Ph}_{2} \mathrm{P}(\mathrm{O}) \mathrm{CHC}(\mathrm{Me}) \mathrm{CH}_{2} \mathrm{Li}(\mathrm{THF})\right\}_{2}\right] 5$

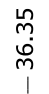

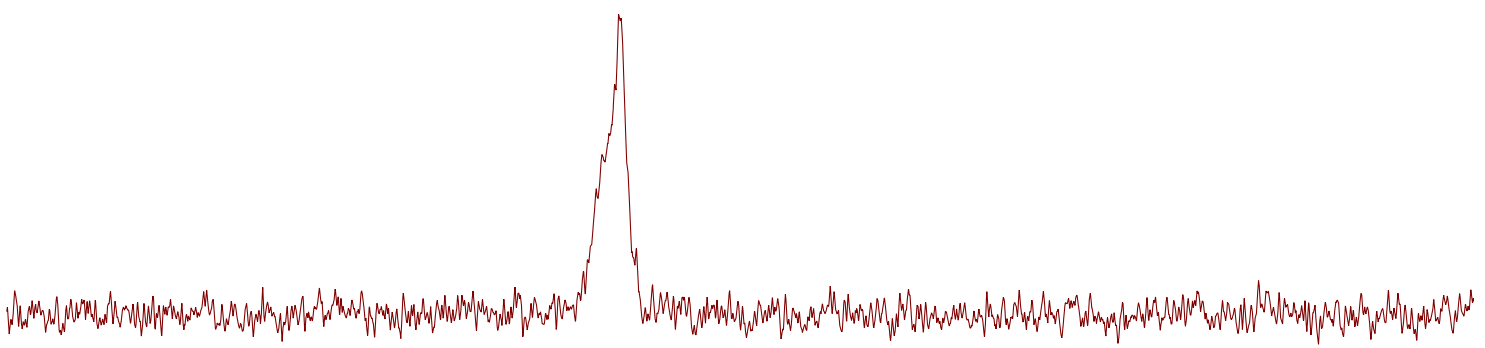

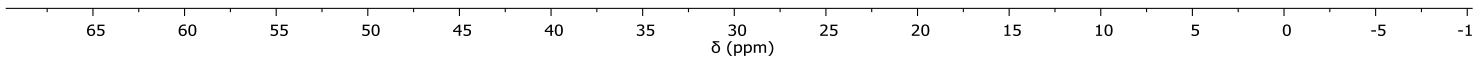

Figure S50. ${ }^{31} \mathrm{P}\{\mathrm{H}\}-\mathrm{NMR}$ (toluene- $\mathrm{d}_{8}, 40{ }^{\circ} \mathrm{C}$ ) of $\left[\left\{\mathrm{Ph}_{2} \mathrm{P}(\mathrm{O}) \mathrm{CHC}(\mathrm{Me}) \mathrm{CH}_{2} \mathrm{Li}(\mathrm{THF})\right\}_{2}\right]$ 

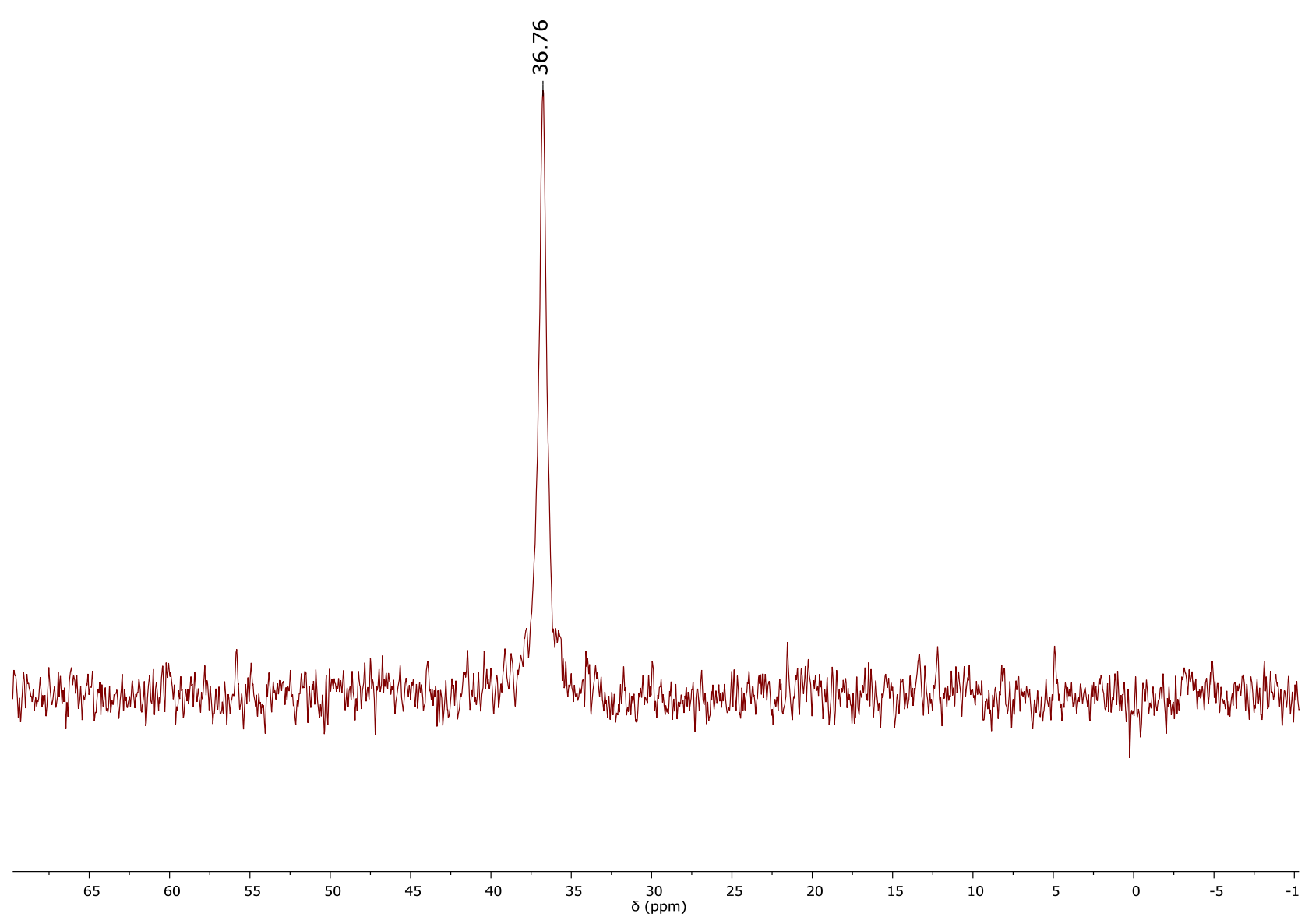

Figure S51. ${ }^{31} \mathrm{P}\{\mathrm{H}\}-\mathrm{NMR}$ (toluene- $\mathrm{d}_{8}, 60{ }^{\circ} \mathrm{C}$ ) of $\left[\left\{\mathrm{Ph}_{2} \mathrm{P}(\mathrm{O}) \mathrm{CHC}(\mathrm{Me}) \mathrm{CH}_{2} \mathrm{Li}(\mathrm{THF})\right\}_{2}\right] 5$

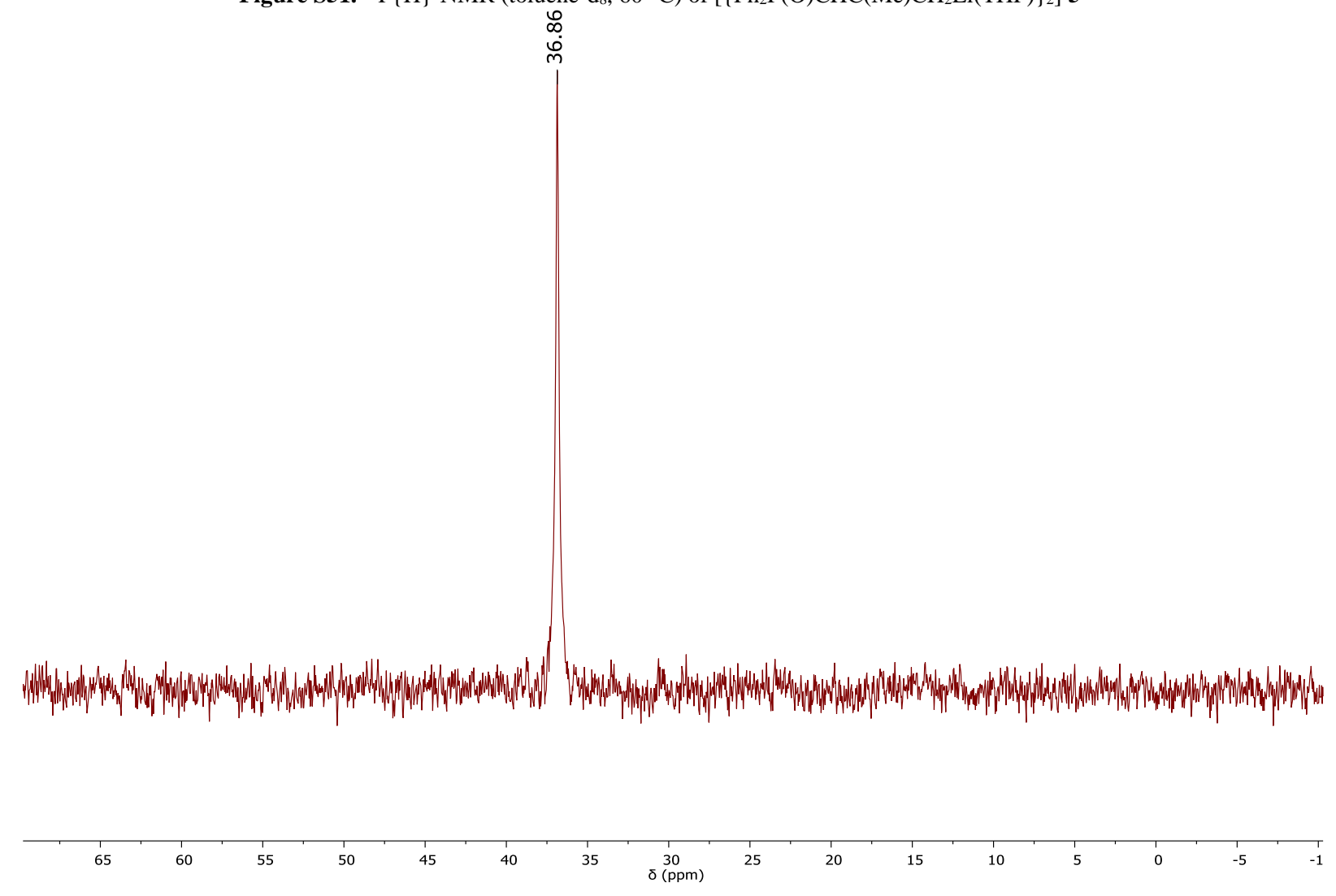

Figure S52. ${ }^{31} \mathrm{P}\{\mathrm{H}\}-\mathrm{NMR}$ (toluene- $\mathrm{d}_{8}, 80{ }^{\circ} \mathrm{C}$ ) of $\left[\left\{\mathrm{Ph}_{2} \mathrm{P}(\mathrm{O}) \mathrm{CHC}(\mathrm{Me}) \mathrm{CH}_{2} \mathrm{Li}(\mathrm{THF})\right\}_{2}\right] 5$

S42 


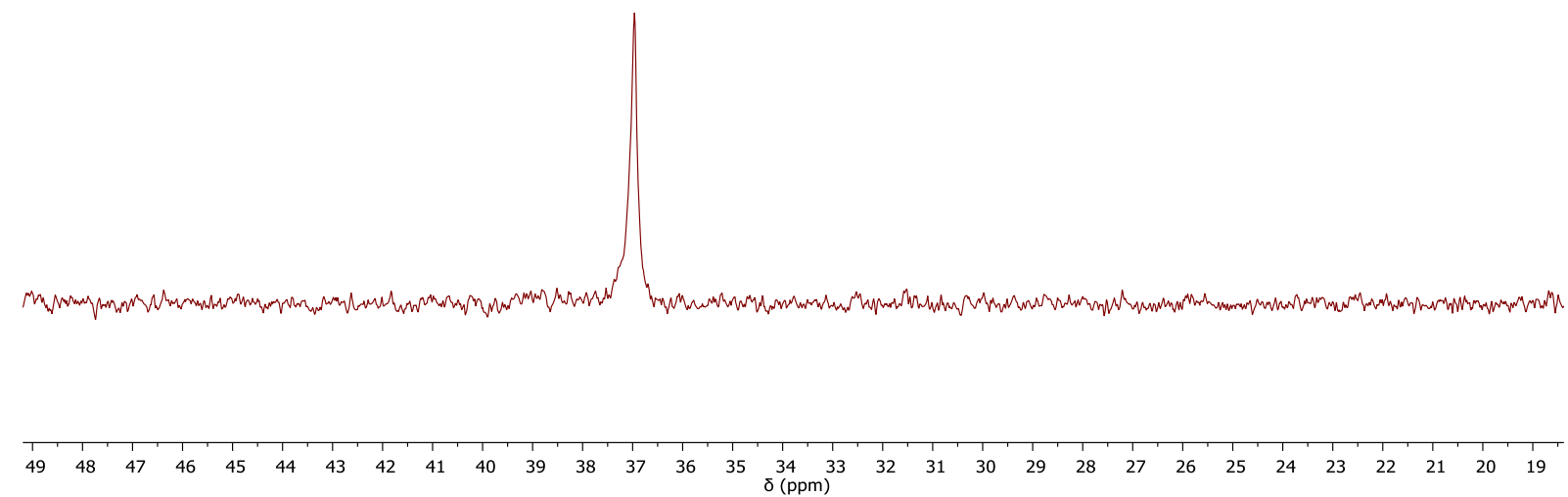

Figure S53. ${ }^{31} \mathrm{P}\{\mathrm{H}\}-\mathrm{NMR}$ (toluene- $\mathrm{d}_{8}, 100{ }^{\circ} \mathrm{C}$ ) of $\left[\left\{\mathrm{Ph}_{2} \mathrm{P}(\mathrm{O}) \mathrm{CHC}(\mathrm{Me}) \mathrm{CH}_{2} \mathrm{Li}(\mathrm{THF})\right\}_{2}\right] 5$

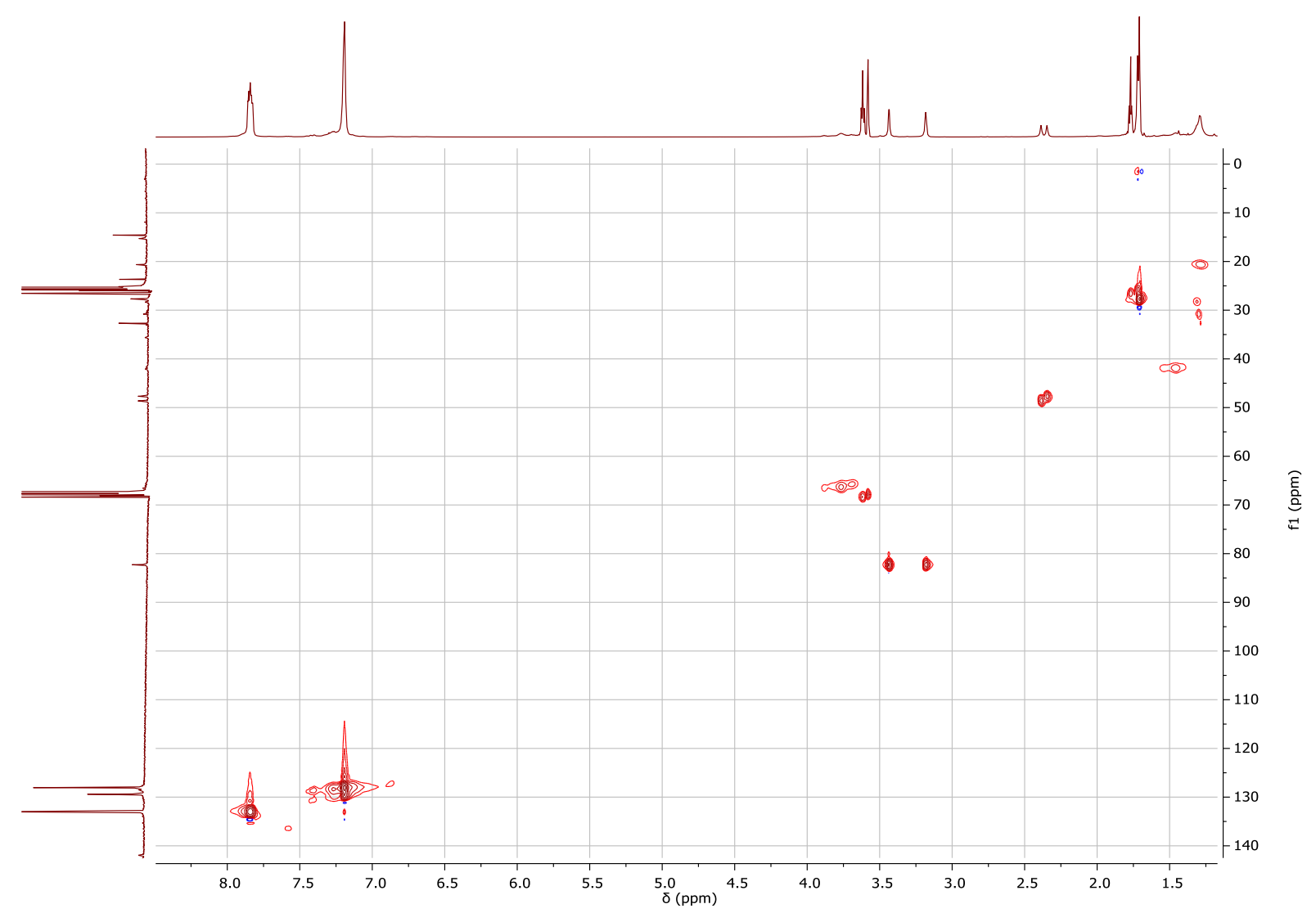

Figure S54. HMQC-NMR (THF- $\left.\mathrm{d}_{8}, 2{ }^{\circ} \mathrm{C}\right)$ of $\left[\left\{\mathrm{Ph} 2 \mathrm{P}(\mathrm{O}) \mathrm{CHC}(\mathrm{Me}) \mathrm{CH}_{2} \mathrm{Li}(\mathrm{THF})\right\}_{2}\right] 5$ 


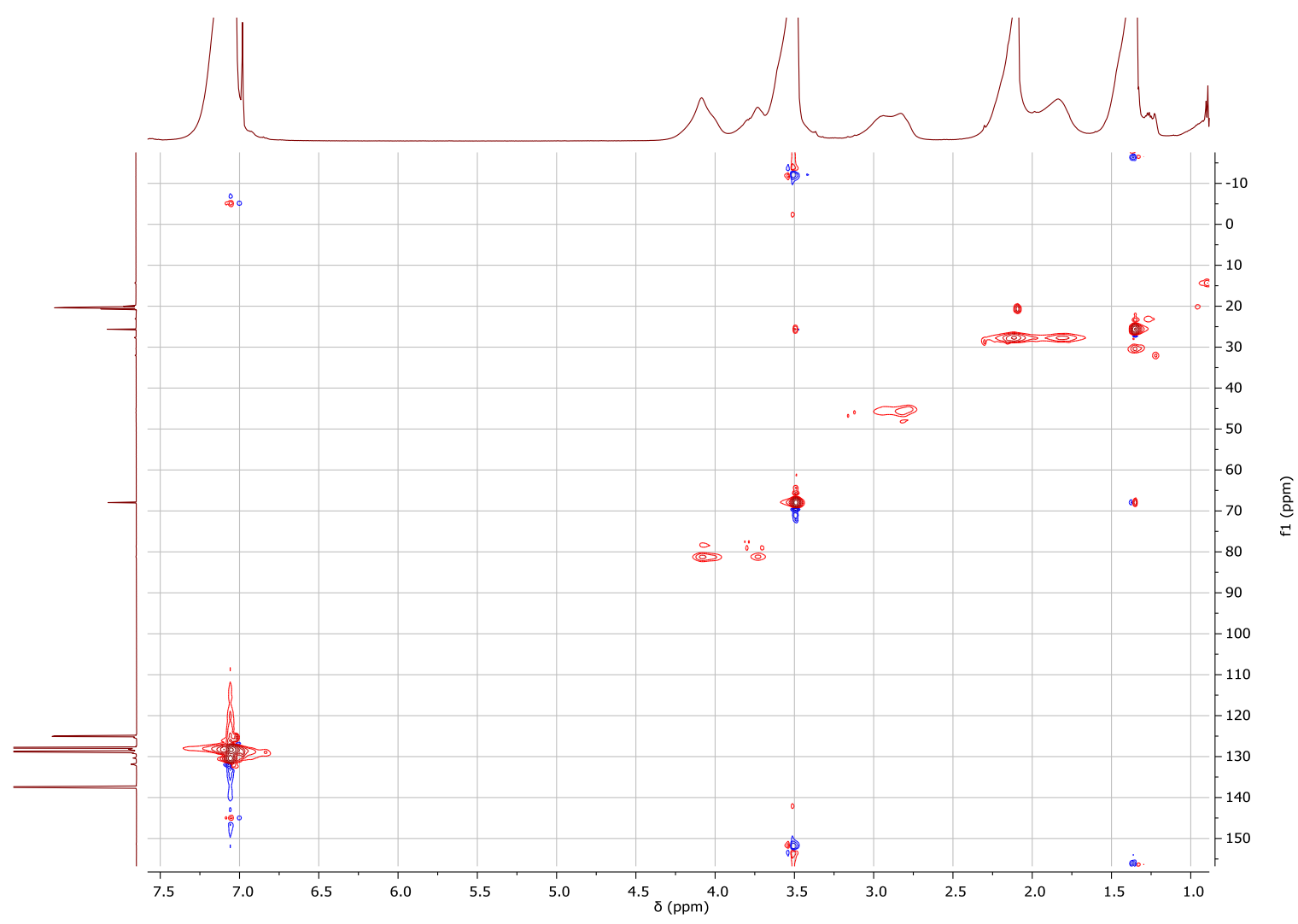

Figure S55. HMQC-NMR (toluene- $\mathrm{d}_{8}, 25^{\circ} \mathrm{C}$ ) of $\left[\left\{\mathrm{Ph}_{2} \mathrm{P}(\mathrm{O}) \mathrm{CHC}(\mathrm{Me}) \mathrm{CH}_{2} \mathrm{Li}(\mathrm{THF})\right\}_{2}\right] 5$

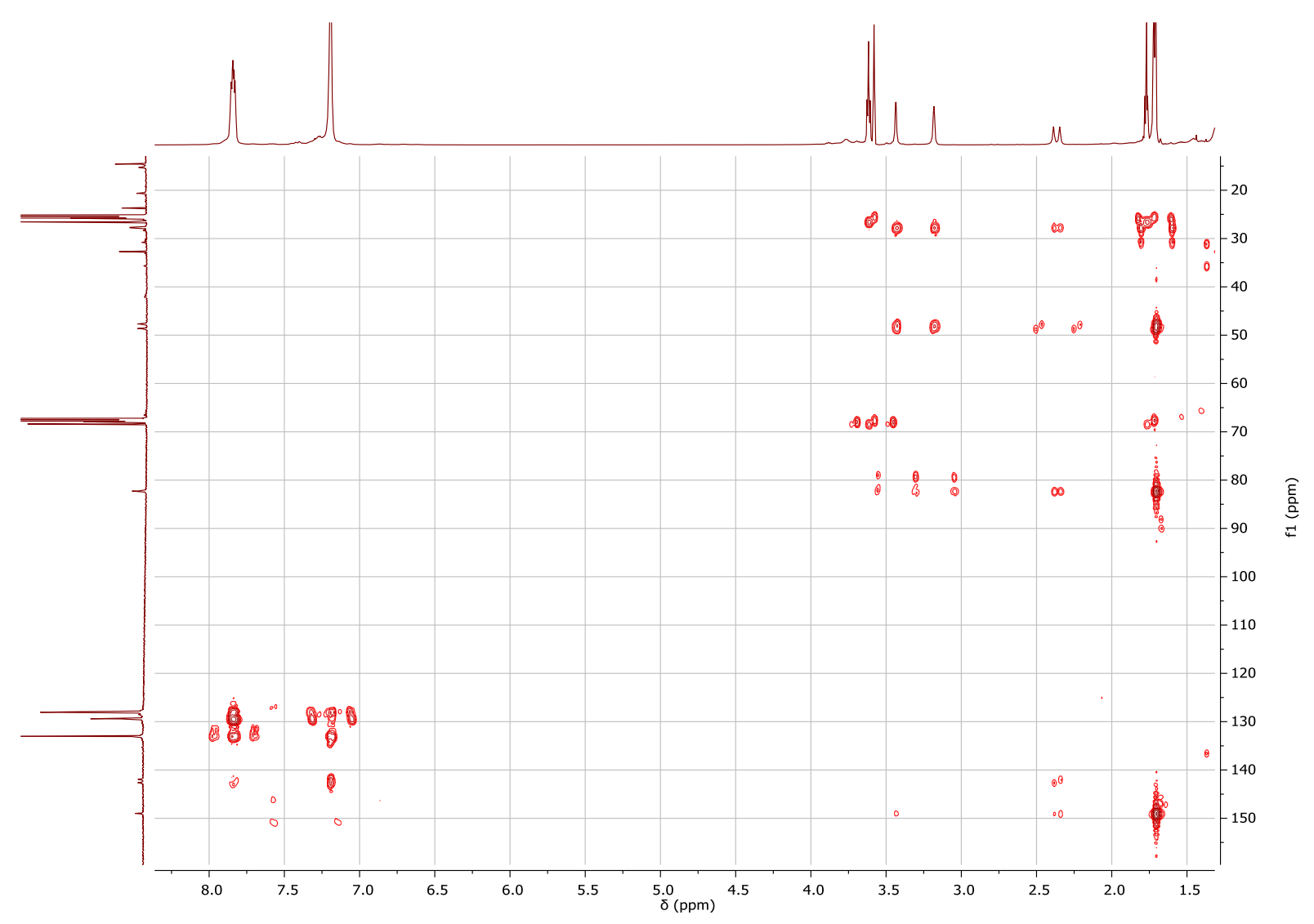

Figure S56. HMBC-NMR (THF-d $\left.8,25^{\circ} \mathrm{C}\right)$ of $\left[\left\{\mathrm{Ph}_{2} \mathrm{P}(\mathrm{O}) \mathrm{CHC}(\mathrm{Me}) \mathrm{CH}_{2} \mathrm{Li}(\mathrm{THF})\right\}_{2}\right] 5$ 


\section{NMR spectra of $\left[\left\{\mathrm{Ph}_{2} \mathrm{P}(\mathrm{O}) \mathrm{CHC}(\mathrm{Me}) \mathrm{CH}_{2} \mathrm{Li}_{2}(\mathrm{TMEDA})\right] 6\right.$ :}

${ }^{1} \mathrm{H}$ NMR (C6 $\mathrm{D}_{6}$ spiked with d8-THF, $400.20 \mathrm{MHz}$ ): $\delta 2.06$ (s, 12H, TMEDA-CH $), 2.18$ (s, 4H, TMEDA-CH $), 2.26(\mathrm{~s}, 6 \mathrm{H}$, $-\mathrm{C}(\mathrm{Me})=), 2.82\left(\mathrm{~d},{ }^{2} J_{\mathrm{H}-\mathrm{P}}=26.5 \mathrm{~Hz}, 2 \mathrm{H}, \mathrm{P}-\mathrm{CH}-\right), 3.90(\mathrm{~s}, 2 \mathrm{H},-\mathrm{C}(\mathrm{Me})=\mathrm{CHH}), 4.24(\mathrm{~s}, 2 \mathrm{H},-\mathrm{C}(\mathrm{Me})=\mathrm{CHH}), 7.16(p-\mathrm{H}), 7.16$ $(m-\mathrm{H}), 8.04(o-\mathrm{H})$.

${ }^{7} \mathrm{Li}$ NMR $\left(\mathrm{C}_{6} \mathrm{D}_{6}\right.$ spiked with $\mathrm{d}_{8}$-THF, $\left.155.53 \mathrm{MHz}\right): \delta-4.18$.

${ }^{13} \mathrm{C}$ NMR $\left(\mathrm{C}_{6} \mathrm{D}_{6}\right.$ spiked with d8-THF, $\left.\left.100.6 \mathrm{MHz}\right): \delta 28.16\left(\mathrm{~d},{ }^{2} J_{\mathrm{C}-\mathrm{P}}=17.9 \mathrm{~Hz},-\mathrm{C}_{2} \mathrm{CH}_{3}\right)=\right), 45.98\left(\mathrm{~s}, \mathrm{TMEDA}-\mathrm{CH}_{3}\right), 46.01$ $\left(\mathrm{d},{ }^{1} J_{\mathrm{C}-\mathrm{P}}=132.5 \mathrm{~Hz}, \mathrm{P}-\mathrm{CH}-\right), 57.84\left(\mathrm{~s}, \mathrm{TMEDA}-\mathrm{CH}_{2}\right), 81.19\left(\mathrm{~m},=\mathrm{CH}_{2}\right), 128.24(\mathrm{~m}, p-\mathrm{C}), 129.91(\mathrm{~s}, m-\mathrm{C}), 132.19\left(\mathrm{~d},{ }^{2} J_{\mathrm{C}-\mathrm{P}}=\right.$ $9.5 \mathrm{~Hz}, o-\mathrm{C}), 138.91\left(\mathrm{~d},{ }^{2} J_{\mathrm{C}-\mathrm{P}}=104.7 \mathrm{~Hz},-\mathrm{C}(\mathrm{Me})=\right), 150.86(\mathrm{~m}, i-\mathrm{C})$.

${ }^{31} \mathrm{P}\left\{{ }^{1} \mathrm{H}\right\}$ NMR (C6 6 spiked with d8-THF, $\left.162.0 \mathrm{MHz}\right): \delta 33.3$.

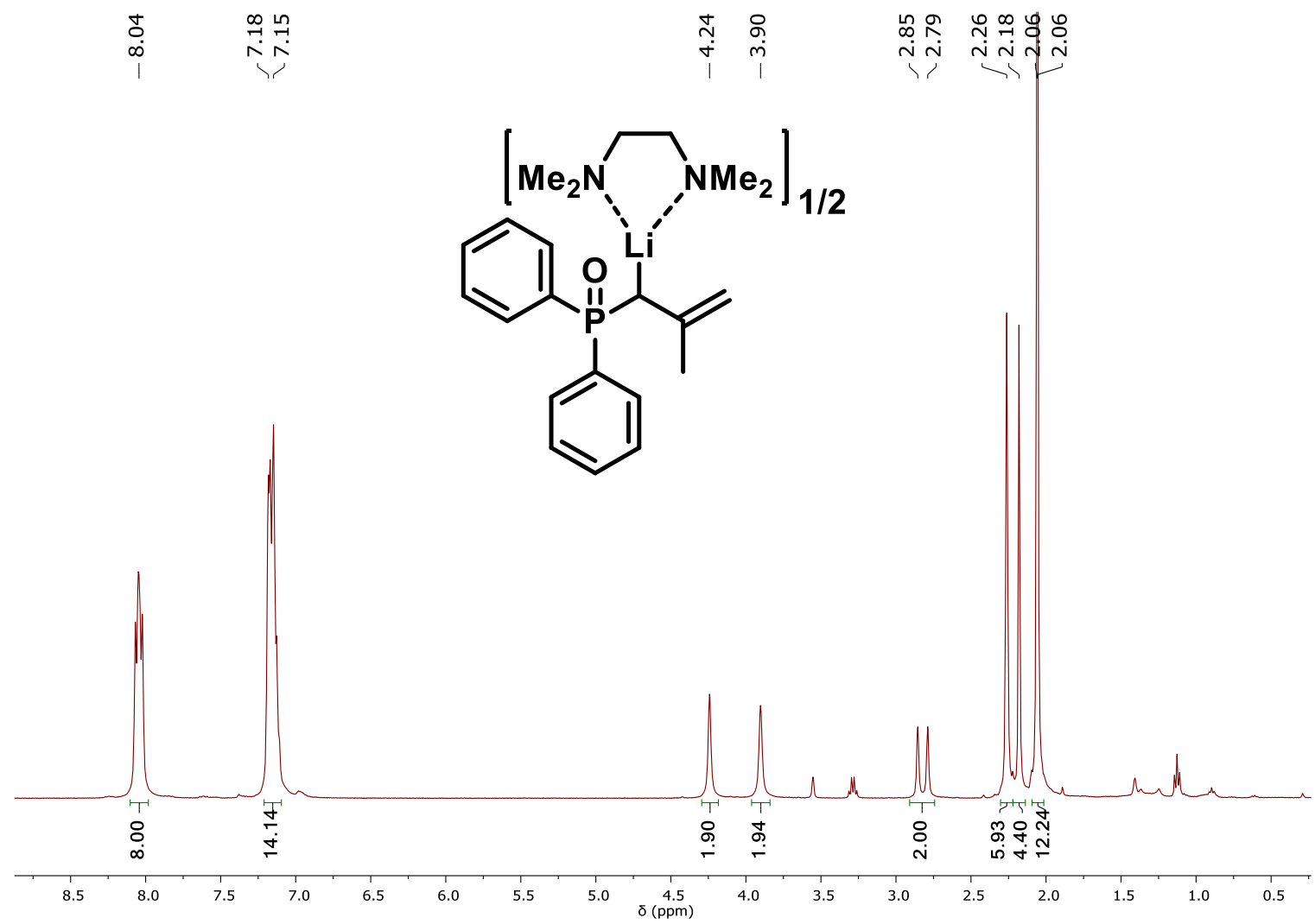

Figure S57. ${ }^{1} \mathrm{H}-\mathrm{NMR}\left(\mathrm{C}_{6} \mathrm{D}_{6}, \mathrm{THF}-\mathrm{d}_{8}\right.$ doped $)$ of $\left[\left\{\mathrm{Ph}_{2} \mathrm{P}(\mathrm{O}) \mathrm{CHC}(\mathrm{Me}) \mathrm{CH}_{2} \mathrm{Li}\right\}_{2}(\mathrm{TMEDA})\right] 6$ 
$-2.2-2.3-2.4-2.5-2.6-2.7-2.8-2.9-3.0-3.1-3.2-3.3-3.4-3.5-3.6-3.7-3.8-3.9-4.0-4.1-4.2-4.3-4.4-4.5-4.6-4.7-4.8-4.9-5.0-5.1-5.2-5.3-5.4-5.5-5.6-5.7-5.8-5.9-6.1$

Figure S58. ${ }^{7} \mathrm{Li}-\mathrm{NMR}\left(\mathrm{C}_{6} \mathrm{D}_{6}, \mathrm{THF}-\mathrm{d}_{8}\right.$ doped $)$ of $\left[\left\{\mathrm{Ph}_{2} \mathrm{P}(\mathrm{O}) \mathrm{CHC}(\mathrm{Me}) \mathrm{CH}_{2} \mathrm{Li}\right\}_{2}(\mathrm{TMEDA})\right] 6$

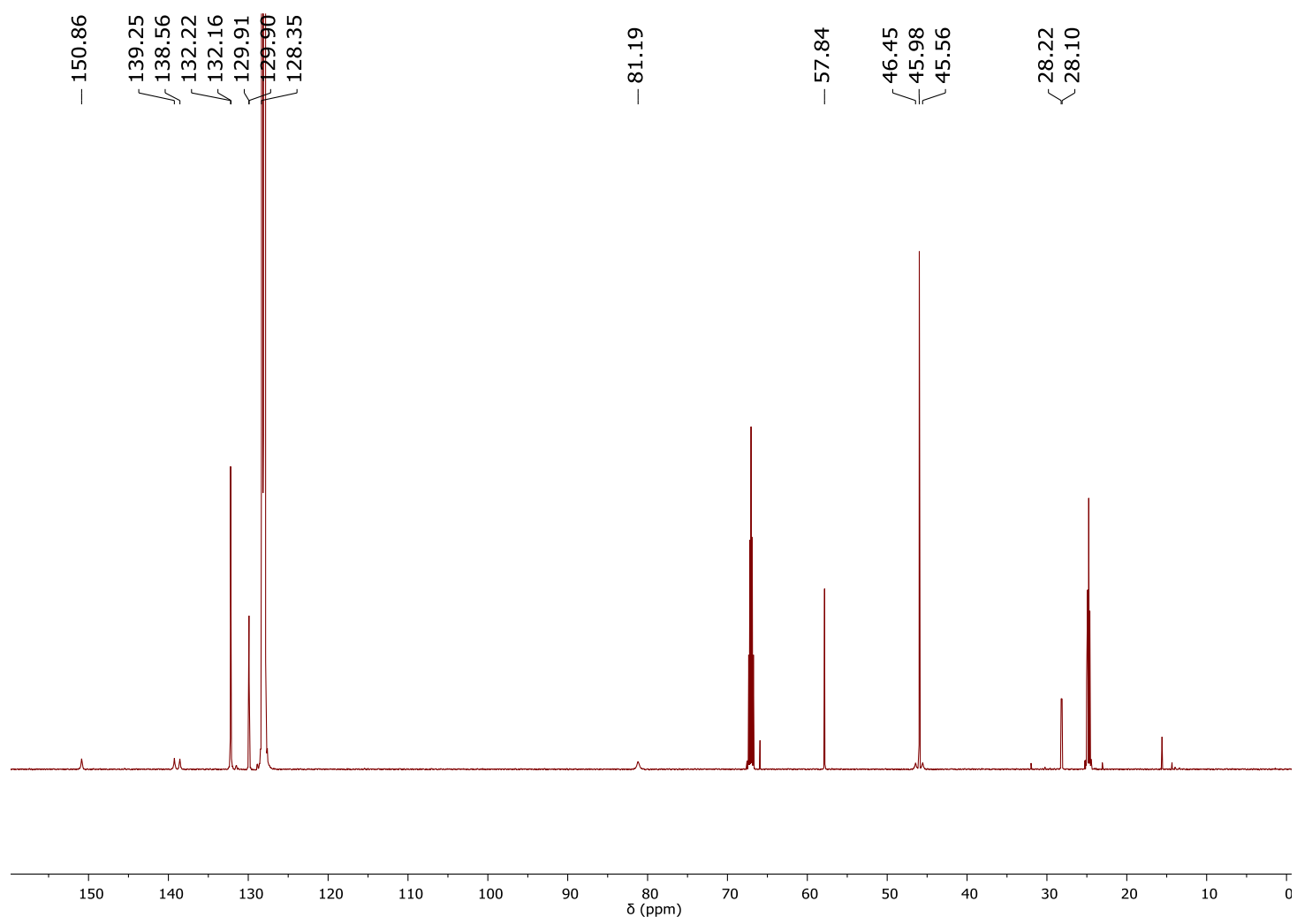

Figure S59. ${ }^{13} \mathrm{C}-\mathrm{NMR}\left(\mathrm{C}_{6} \mathrm{D}_{6}, \mathrm{THF}-\mathrm{d}_{8}\right.$ doped $)$ of $\left[\left\{\mathrm{Ph}_{2} \mathrm{P}(\mathrm{O}) \mathrm{CHC}(\mathrm{Me}) \mathrm{CH}_{2} \mathrm{Li}\right\}_{2}\right.$ (TMEDA) 6 


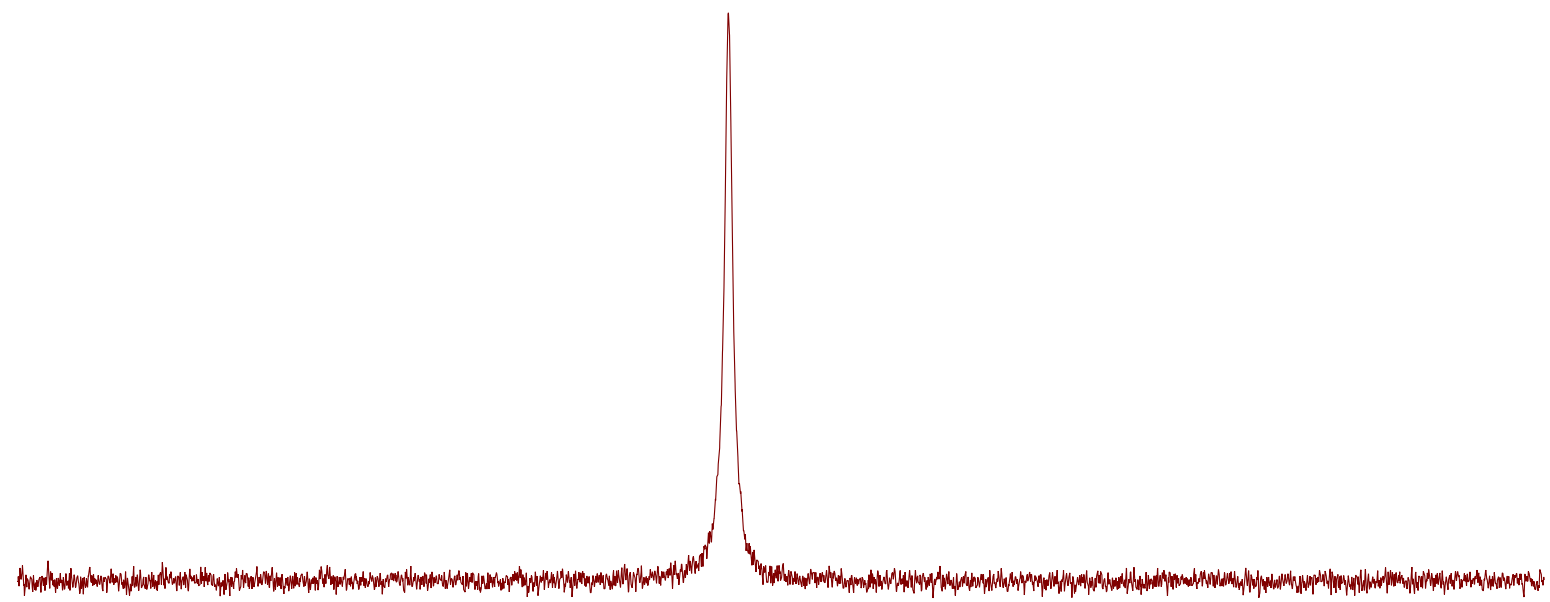

$\begin{array}{lllllllll}1 & 1 & 1 & 1 & 1 & 10 & 10 & 10 & 10\end{array}$

Figure S60. ${ }^{31} \mathrm{P}\left\{{ }^{1} \mathrm{H}\right\}-\mathrm{NMR}\left(\mathrm{C}_{6} \mathrm{D}_{6}, \mathrm{THF}-\mathrm{d}_{8}\right.$ doped $)$ of $\left[\left\{\mathrm{Ph}_{2} \mathrm{P}(\mathrm{O}) \mathrm{CHC}(\mathrm{Me}) \mathrm{CH}_{2} \mathrm{Li}\right\}_{2}(\mathrm{TMEDA})\right] 6$

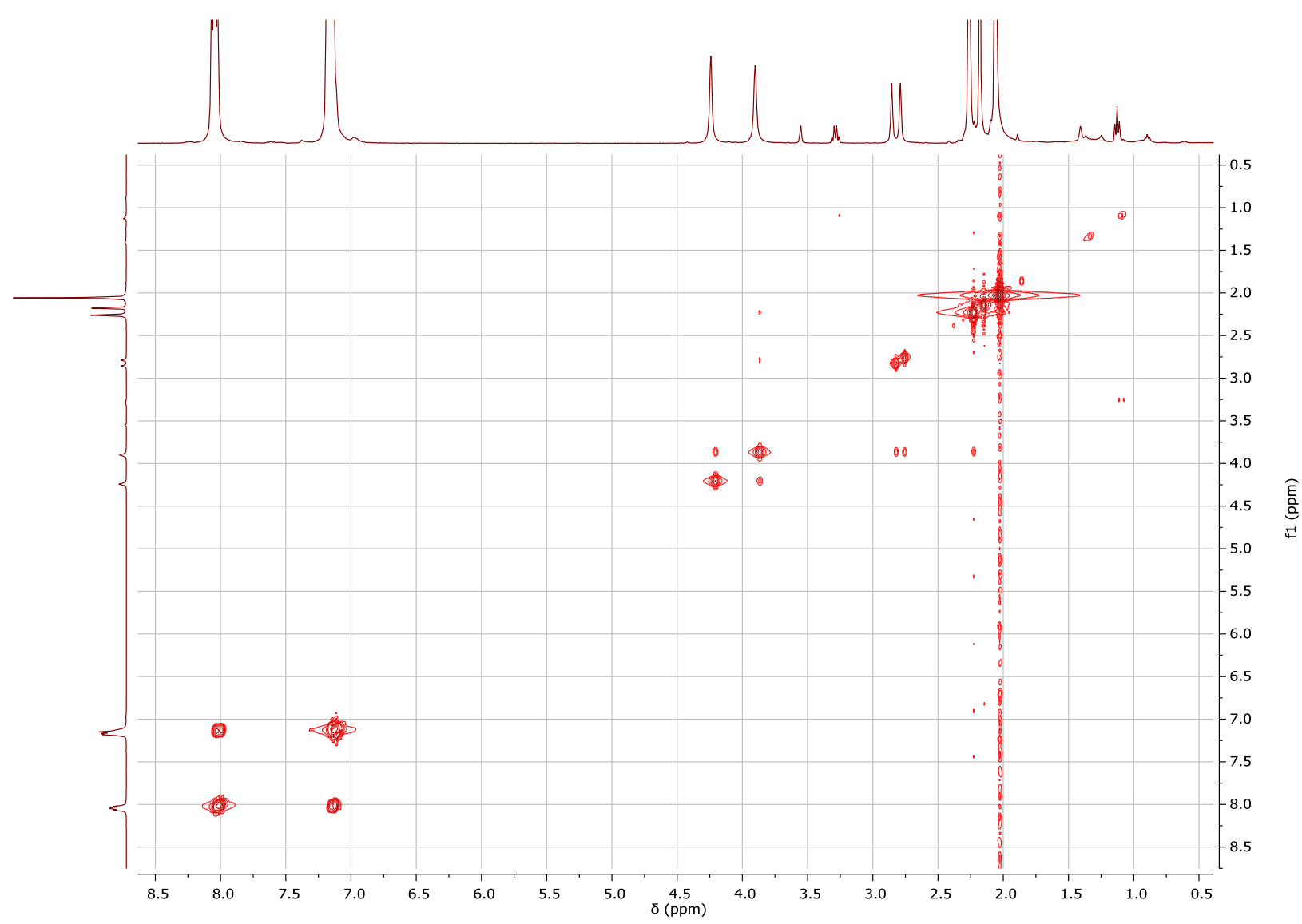

Figure S61. ${ }^{1} \mathrm{H}-{ }^{1} \mathrm{H}$ COSY-NMR $\left(\mathrm{C}_{6} \mathrm{D}_{6}\right.$, THF-d 8 doped $)$ of $\left[\left\{\mathrm{Ph}_{2} \mathrm{P}(\mathrm{O}) \mathrm{CHC}(\mathrm{Me}) \mathrm{CH}_{2} \mathrm{Li}_{2}(\mathrm{TMEDA})\right] 6\right.$ 


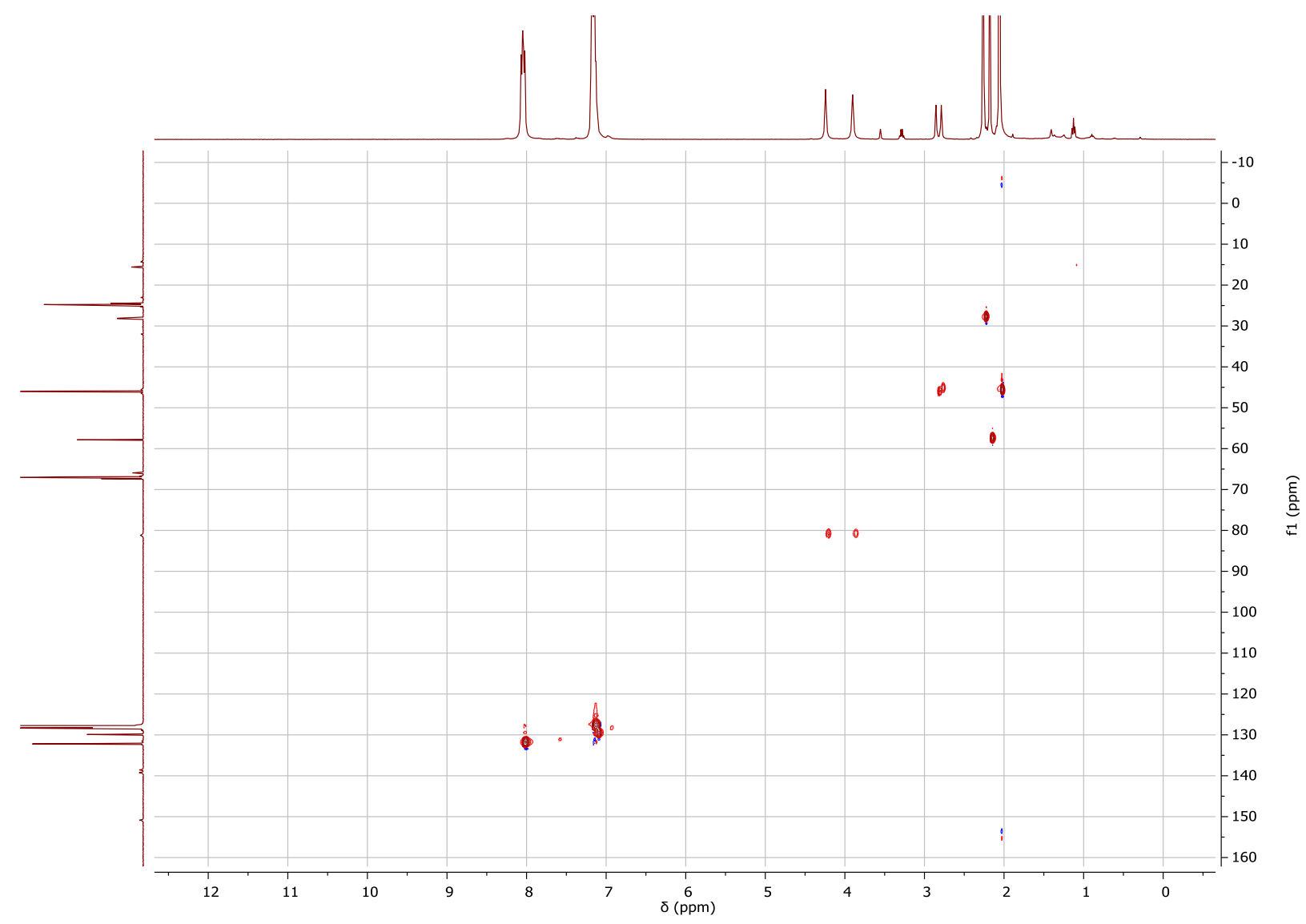

Figure S62. HMQC-NMR $\left(\mathrm{C}_{6} \mathrm{D}_{6}\right.$, THF- $\mathrm{d}_{8}$ doped $)$ of $\left[\left\{\mathrm{Ph}_{2} \mathrm{P}(\mathrm{O}) \mathrm{CHC}(\mathrm{Me}) \mathrm{CH}_{2} \mathrm{Li}_{2}\right\}_{2}(\mathrm{TMEDA})\right] 6$ 


\section{NMR spectra of [Ph $\left.2 \mathrm{P}(\mathrm{O}) \mathrm{CHC}(\mathrm{Me}) \mathrm{CH}_{2} \mathrm{Li}(\mathrm{PMDETA})\right]$ 7:}

${ }^{1} \mathrm{H}$ NMR $\left(\mathrm{C}_{6} \mathrm{D}_{6}, 400.20 \mathrm{MHz}\right): \delta 1.80-2.20(\mathrm{~m}, 23 \mathrm{H}, \mathrm{PMDETA}), 2.36(\mathrm{~s}, 3 \mathrm{H},-\mathrm{C}(\mathrm{Me})=), 3.05\left(\mathrm{~d},{ }^{3} J_{\mathrm{H}-\mathrm{P}}=24.9 \mathrm{~Hz}, 1 \mathrm{H}, \mathrm{P}-\mathrm{CH}-\right.$ ), $3.96(\mathrm{~s}, 1 \mathrm{H},=\mathrm{CHH}), 4.05(\mathrm{~s}, 1 \mathrm{H},=\mathrm{CHH}), 7.13(p-\mathrm{H}), 7.25(\mathrm{~m}-\mathrm{H}), 8.32(o-\mathrm{H})$.

${ }^{7} \mathrm{Li}$ NMR ( $\left.\mathrm{C}_{6} \mathrm{D}_{6}, 155.53 \mathrm{MHz}\right): \delta$-3.99.

${ }^{13} \mathrm{C}$ NMR $\left(\mathrm{C}_{6} \mathrm{D}_{6}, 100.6 \mathrm{MHz}\right): \delta 28.31\left(\mathrm{~d},{ }^{2} J_{\mathrm{C}-\mathrm{P}}=17.6 \mathrm{~Hz},-\mathrm{C}\left(\mathrm{CH}_{3}\right)=\right), 44.15$ (s, PMDETA), 45.58 (s, PMDETA), $48.52(\mathrm{~d}$, $\left.{ }^{1} J_{\mathrm{C}-\mathrm{P}}=142.1 \mathrm{~Hz}, \mathrm{P}-\mathrm{CH}-\right), 53.47$ (s, PMDETA), 57.20 (s, PMDETA), $81.78\left(\mathrm{~d},{ }^{3} J_{\mathrm{C}-\mathrm{P}}=6.6 \mathrm{~Hz},=\mathrm{CH}_{2}\right), 127.80(\mathrm{~m}, m-\mathrm{C})$, $128.90(\mathrm{~s}, p-\mathrm{C}), 132.55\left(\mathrm{~d},{ }^{2} J_{\mathrm{C}-\mathrm{P}}=8.7 \mathrm{~Hz}, o-\mathrm{C}\right), 142.52\left(\mathrm{~d},{ }^{1} J_{\mathrm{C}-\mathrm{P}}=105.0 \mathrm{~Hz},-\mathrm{C}(\mathrm{Me})=\right), 149.23\left(\mathrm{~d},{ }^{2} J_{\mathrm{C}-\mathrm{P}}=7.1 \mathrm{~Hz}, i-\mathrm{C}\right)$.

${ }^{31} \mathrm{P}\left\{{ }^{1} \mathrm{H}\right\}$ NMR $\left(\mathrm{C}_{6} \mathrm{D}_{6}, 162.0 \mathrm{MHz}\right): \delta 26.1$.

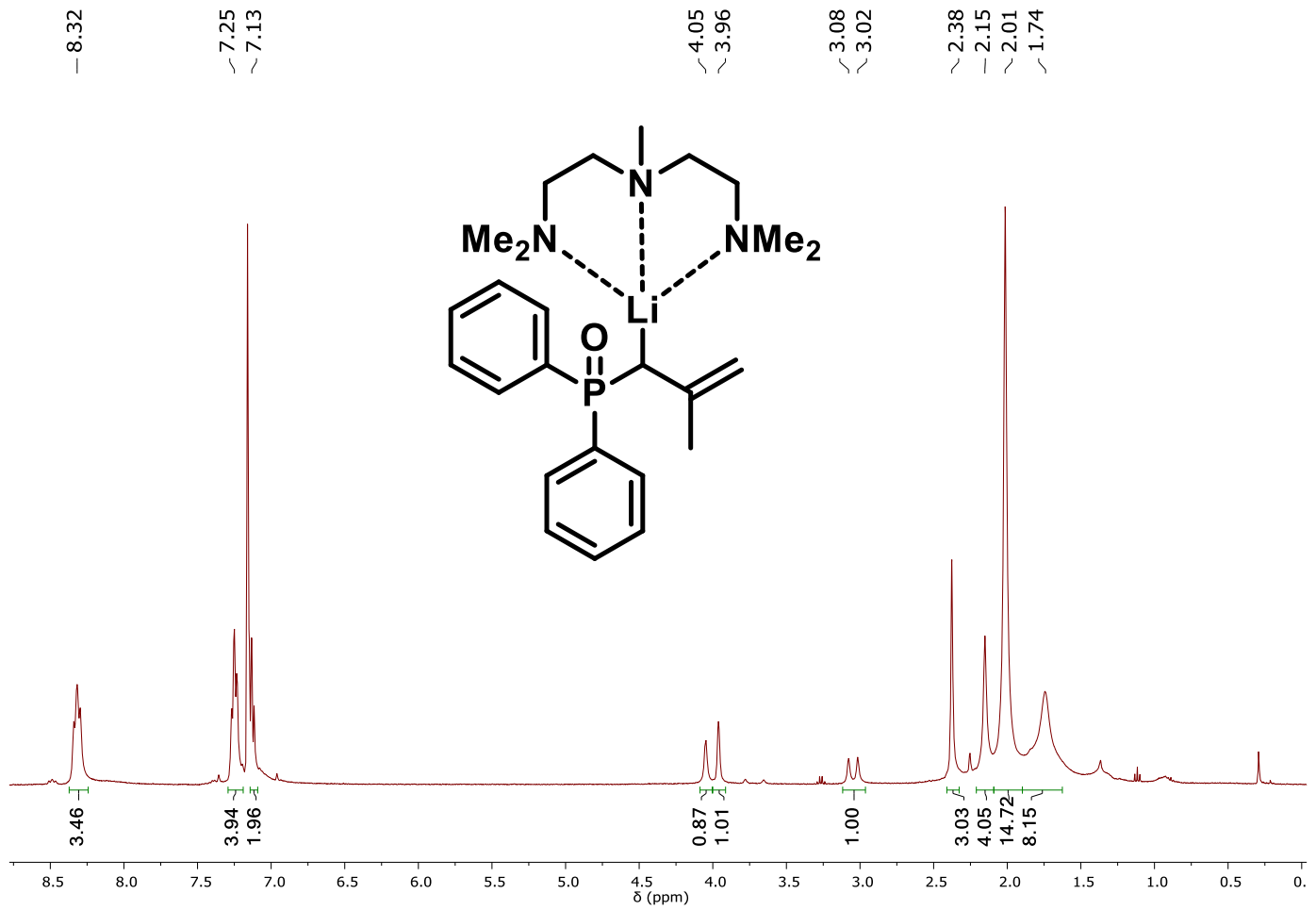

Figure S63. ${ }^{1} \mathrm{H}-\mathrm{NMR}\left(\mathrm{C}_{6} \mathrm{D}_{6}\right)$ of $\left[\mathrm{Ph}_{2} \mathrm{P}(\mathrm{O}) \mathrm{CHC}(\mathrm{Me}) \mathrm{CH}_{2} \mathrm{Li}(\mathrm{PMDETA})\right] 7$ 


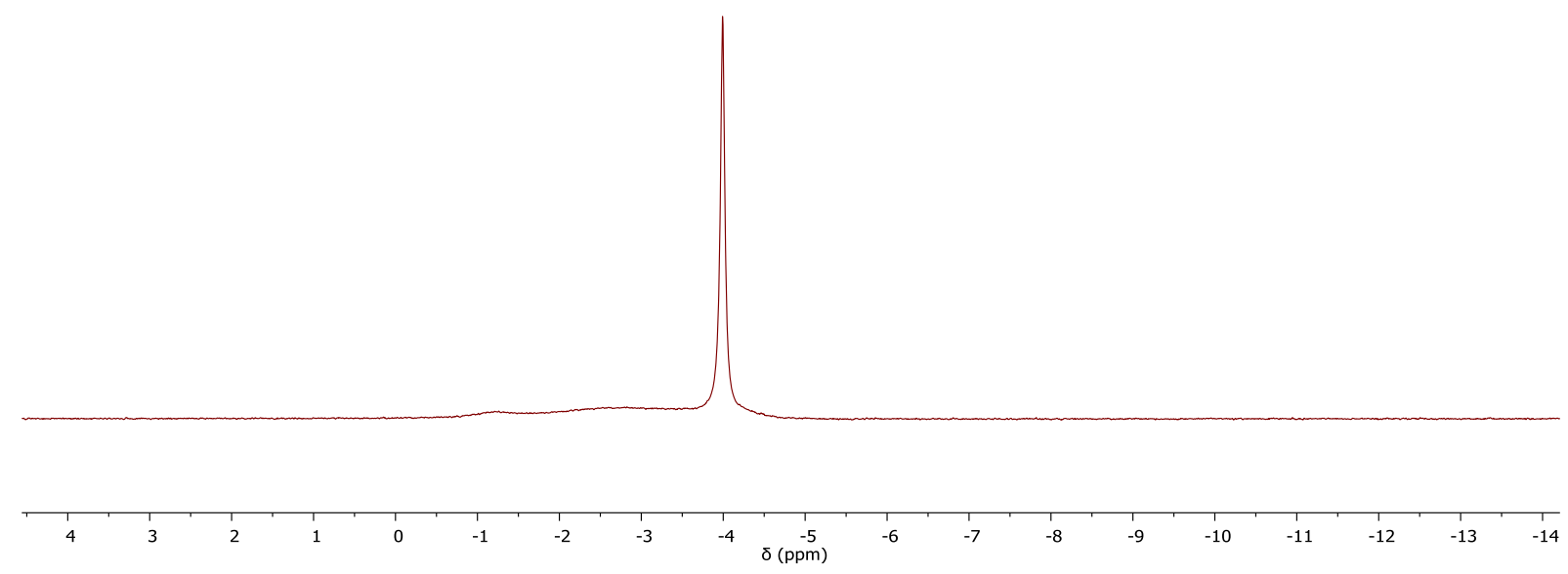

Figure S64. ${ }^{7} \mathrm{Li}-\mathrm{NMR}\left(\mathrm{C}_{6} \mathrm{D}_{6}\right)$ of $\left[\mathrm{Ph}_{2} \mathrm{P}(\mathrm{O}) \mathrm{CHC}(\mathrm{Me}) \mathrm{CH}_{2} \mathrm{Li}(\mathrm{PMDETA})\right] 7$

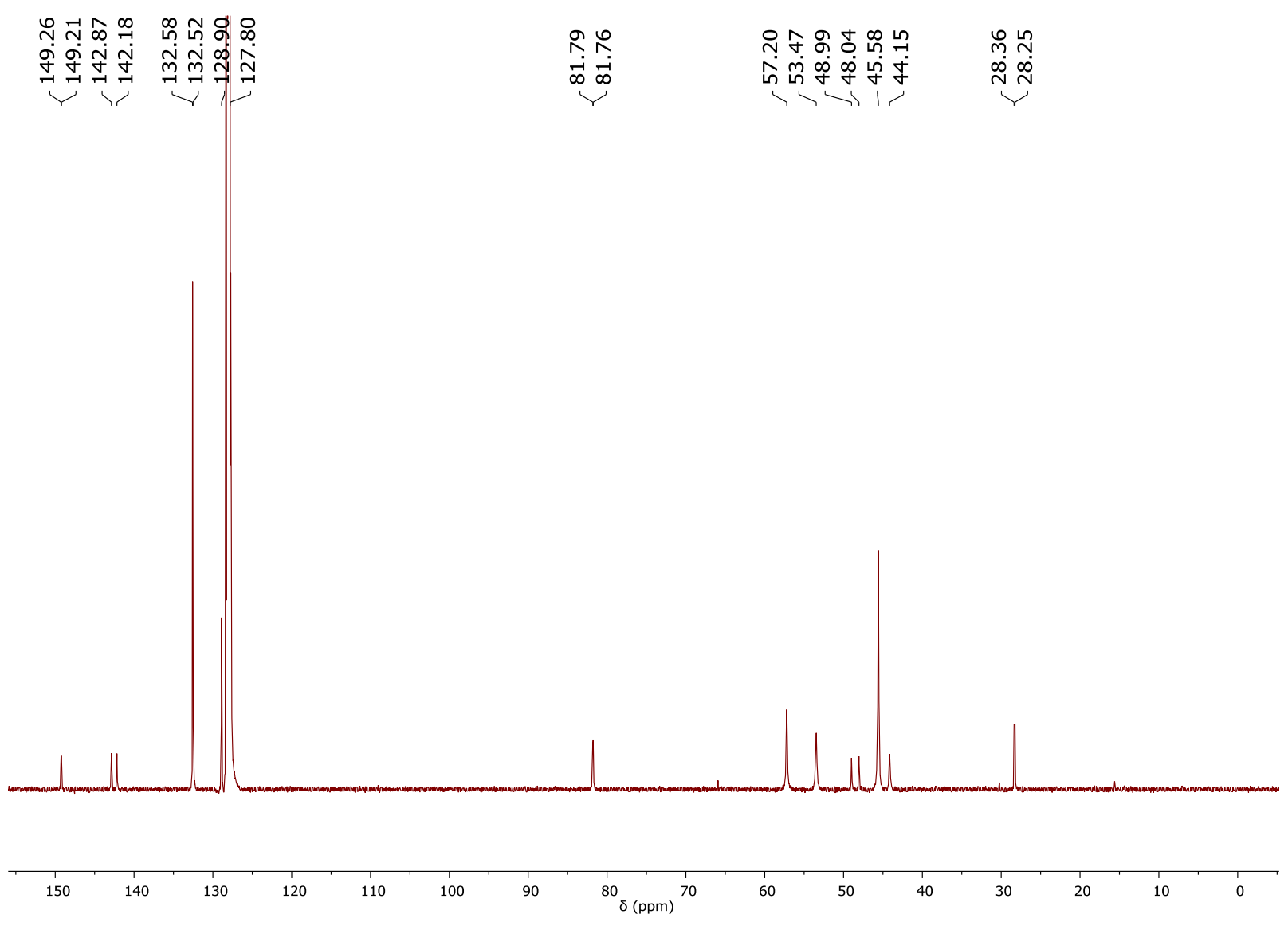

Figure S65. ${ }^{13} \mathrm{C}-\mathrm{NMR}\left(\mathrm{C}_{6} \mathrm{D}_{6}\right)$ of $\left[\mathrm{Ph}_{2} \mathrm{P}(\mathrm{O}) \mathrm{CHC}(\mathrm{Me}) \mathrm{CH}_{2} \mathrm{Li}(\mathrm{PMDETA})\right] 7$ 


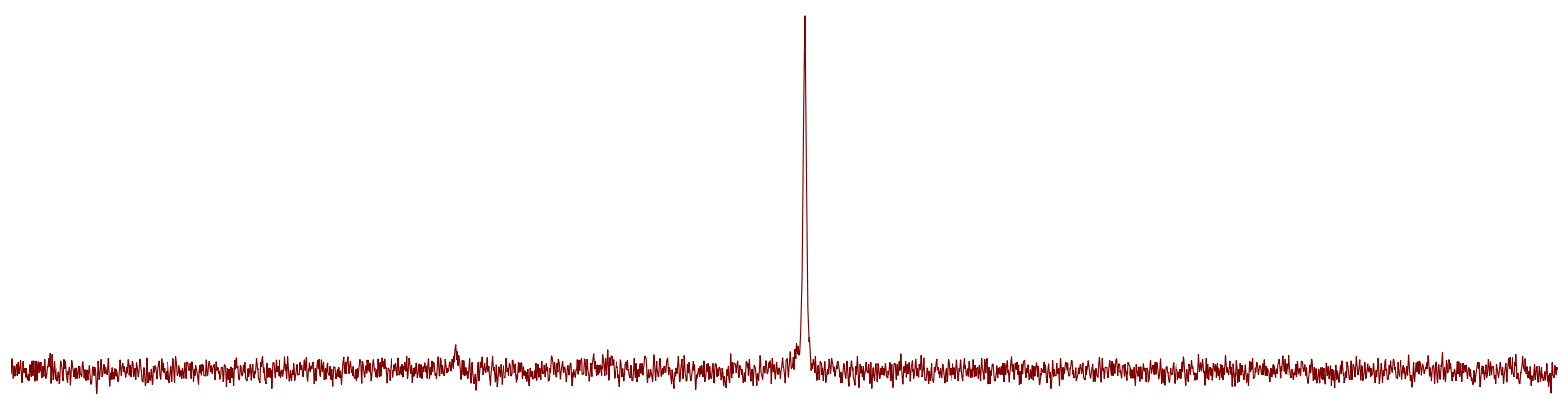

Figure S66. ${ }^{31} \mathrm{P}\{\mathrm{H}\}-\mathrm{NMR}\left(\mathrm{C}_{6} \mathrm{D}_{6}\right)$ of $\left[\mathrm{Ph}_{2} \mathrm{P}(\mathrm{O}) \mathrm{CHC}(\mathrm{Me}) \mathrm{CH}_{2} \mathrm{Li}(\mathrm{PMDETA})\right] 7$

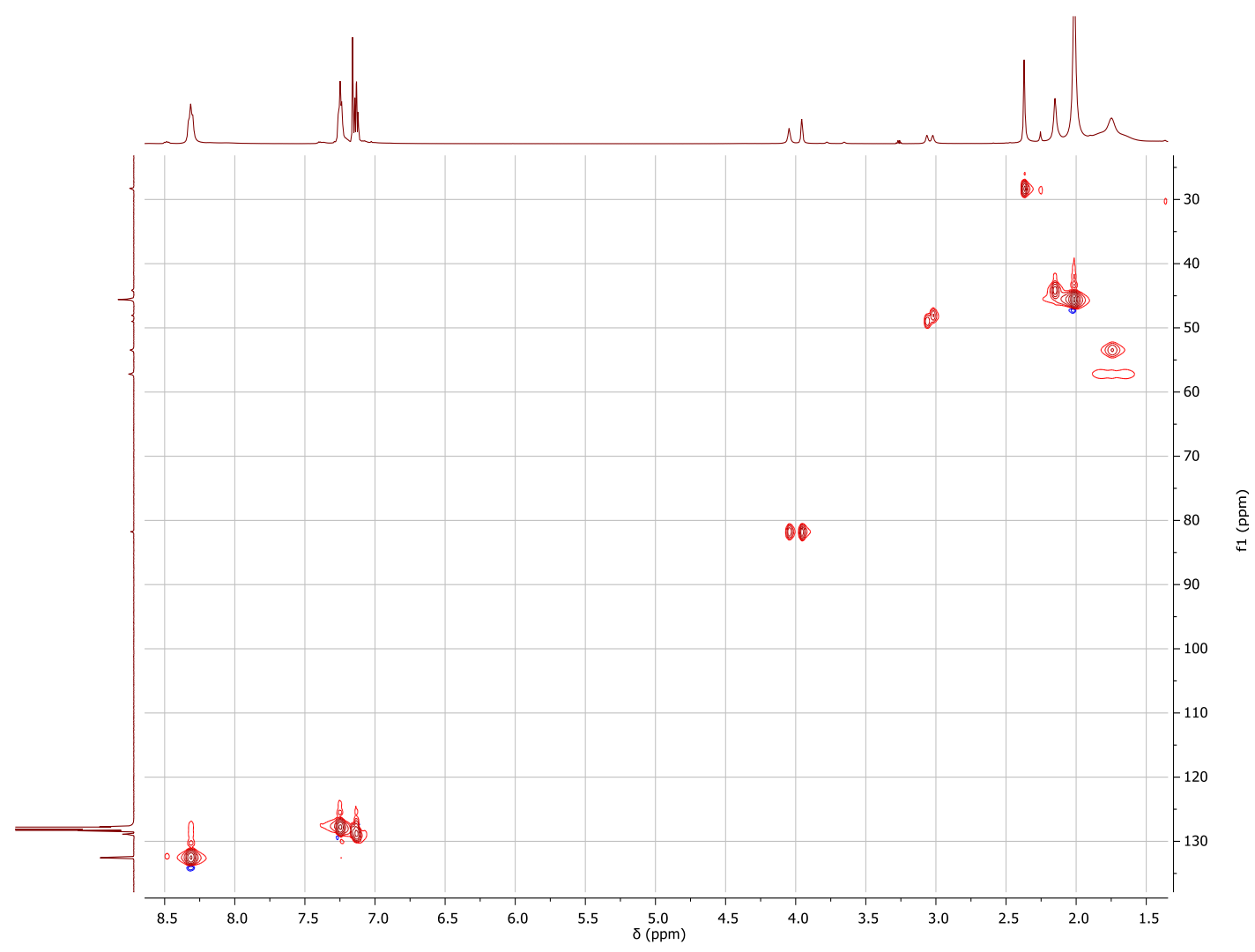

Figure S67. HMQC-NMR $\left(\mathrm{C}_{6} \mathrm{D}_{6}\right)$ of $\left[\mathrm{Ph}_{2} \mathrm{P}(\mathrm{O}) \mathrm{CHC}(\mathrm{Me}) \mathrm{CH}_{2} \mathrm{Li}(\mathrm{PMDETA})\right] 7$ 


\section{NMR spectra of $\left[\mathrm{Ph}_{2} \mathrm{P}(\mathrm{O}) \mathrm{CHCHCMe} 2 \mathrm{Li}(\mathrm{PMDETA})\right]$ 8:}

${ }^{1} \mathrm{H}$ NMR (C6 $\left.\mathrm{D}_{6}, 400.20 \mathrm{MHz}\right): \delta 1.61-2.17$ (m, 23H, PMDETA), 2.17 (br-s, 6H, =CMe $), 3.16$ (dd, ${ }^{3} J_{\mathrm{H}-\mathrm{P}}=20.0 \mathrm{~Hz}, \mathrm{JHH}=$ $12.7 \mathrm{~Hz}, 1 \mathrm{H}, \mathrm{P}-\mathrm{CH}-), 6.24\left(\mathrm{dd},{ }^{3} J_{\mathrm{H}-\mathrm{P}}=13.0 \mathrm{~Hz}, J_{\mathrm{H}-\mathrm{H}}=12.7 \mathrm{~Hz}, 1 \mathrm{H},-\mathrm{CH}=\right), 7.11(\mathrm{~m}, 2 \mathrm{H}, p-\mathrm{H}), 7.23(\mathrm{~m}, 4 \mathrm{H}, m-\mathrm{H}), 8.41(\mathrm{~m}$, $4 \mathrm{H}, o-\mathrm{H})$.

${ }^{7} \mathrm{Li}$ NMR $\left(\mathrm{C}_{6} \mathrm{D}_{6}, 155.53 \mathrm{MHz}\right): \delta$-3.94.

${ }^{13} \mathrm{C}$ NMR $\left(\mathrm{C}_{6} \mathrm{D}_{6}, 100.6 \mathrm{MHz}\right): \delta 19.02\left(\mathrm{~s},=\mathrm{C}\left(\mathrm{CH}_{3}\right)_{2}\right), 27.05\left(\mathrm{~s},=\mathrm{C}\left(\mathrm{CH}_{3}\right)_{2}\right), 44.20$ (s, PMDETA), $44.21\left(\mathrm{~d},{ }^{1} J_{\mathrm{C}-\mathrm{P}}=147.2 \mathrm{~Hz}\right.$, P-CH-), 45.60 (s, PMDETA), 53.43 (s, PMDETA), 57.12 (s, PMDETA), 96.61 (d, $\left.{ }^{3} J_{\mathrm{C}-\mathrm{P}}=21.3 \mathrm{~Hz},=\mathrm{CMe}_{2}\right) 127.61\left(\mathrm{~d},{ }^{3} J_{\mathrm{C}-\mathrm{P}}\right.$ $=10.6 \mathrm{~Hz}, m-\mathrm{C}), 128.90(\mathrm{~s}, p-\mathrm{C}), 129.49\left(\mathrm{~d},{ }^{2} J_{\mathrm{C}-\mathrm{P}}=9.1 \mathrm{~Hz},-\mathrm{CH}=\right), 133.41\left(\mathrm{~d},{ }^{2} J_{\mathrm{C}-\mathrm{P}}=8.2 \mathrm{~Hz}, o-\mathrm{C}\right), 142.90\left(\mathrm{~d},{ }^{1} J_{\mathrm{C}-\mathrm{P}}=106.8\right.$ $\mathrm{Hz}, i-\mathrm{C})$

${ }^{31} \mathrm{P}\left\{{ }^{1} \mathrm{H}\right\}$ NMR $\left(\mathrm{C}_{6} \mathrm{D}_{6}, 162.0 \mathrm{MHz}\right): \delta 28.9$.

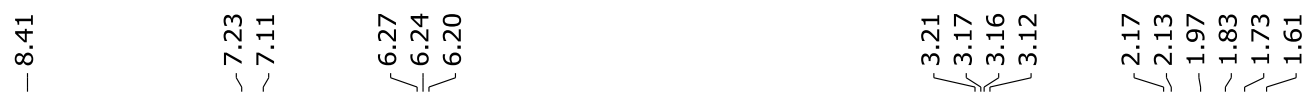

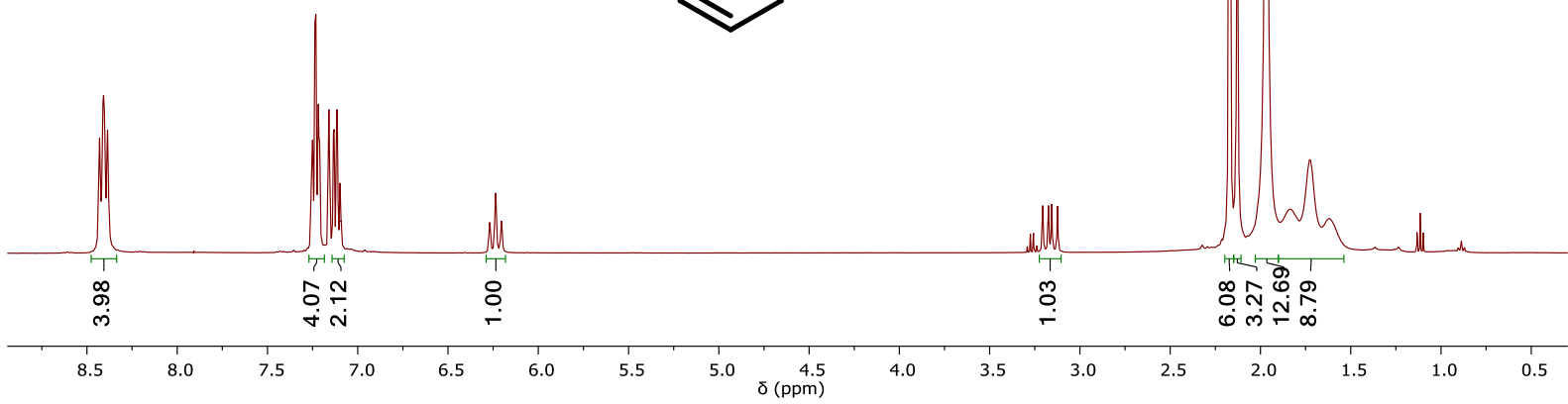

Figure S68. ${ }^{1} \mathrm{H}-\mathrm{NMR}\left(\mathrm{C}_{6} \mathrm{D}_{6}\right)$ of $\left[\mathrm{Ph}_{2} \mathrm{P}(\mathrm{O}) \mathrm{CHCHCMe}_{2} \mathrm{Li}(\mathrm{PMDETA})\right] \mathrm{8}$ 


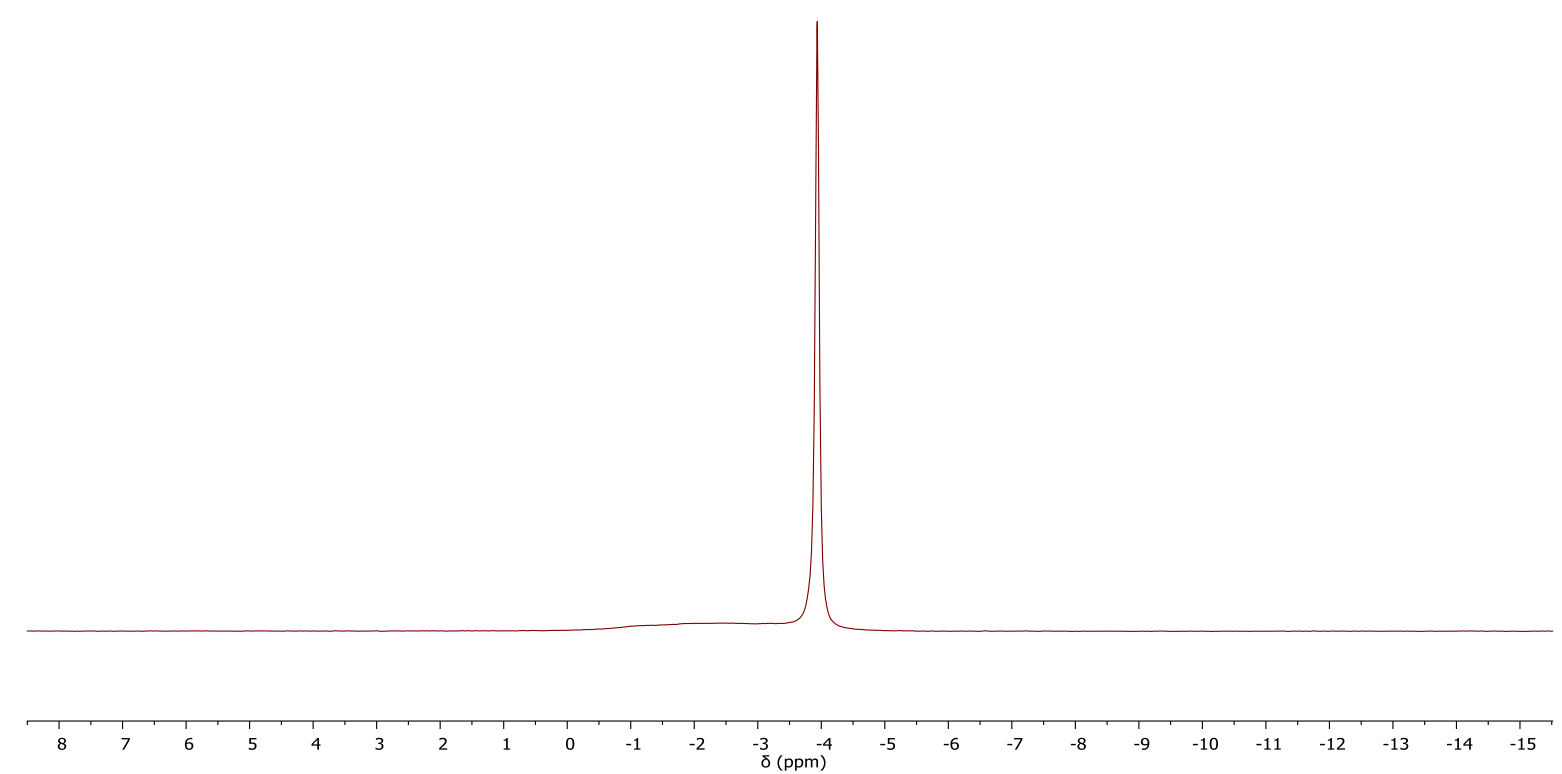

Figure S69. ${ }^{7} \mathrm{Li}-\mathrm{NMR}\left(\mathrm{C}_{6} \mathrm{D}_{6}\right)$ of $\left[\mathrm{Ph}_{2} \mathrm{P}(\mathrm{O}) \mathrm{CHCHCMe} 2 \mathrm{Li}(\mathrm{PMDETA})\right] \textrm{ }$

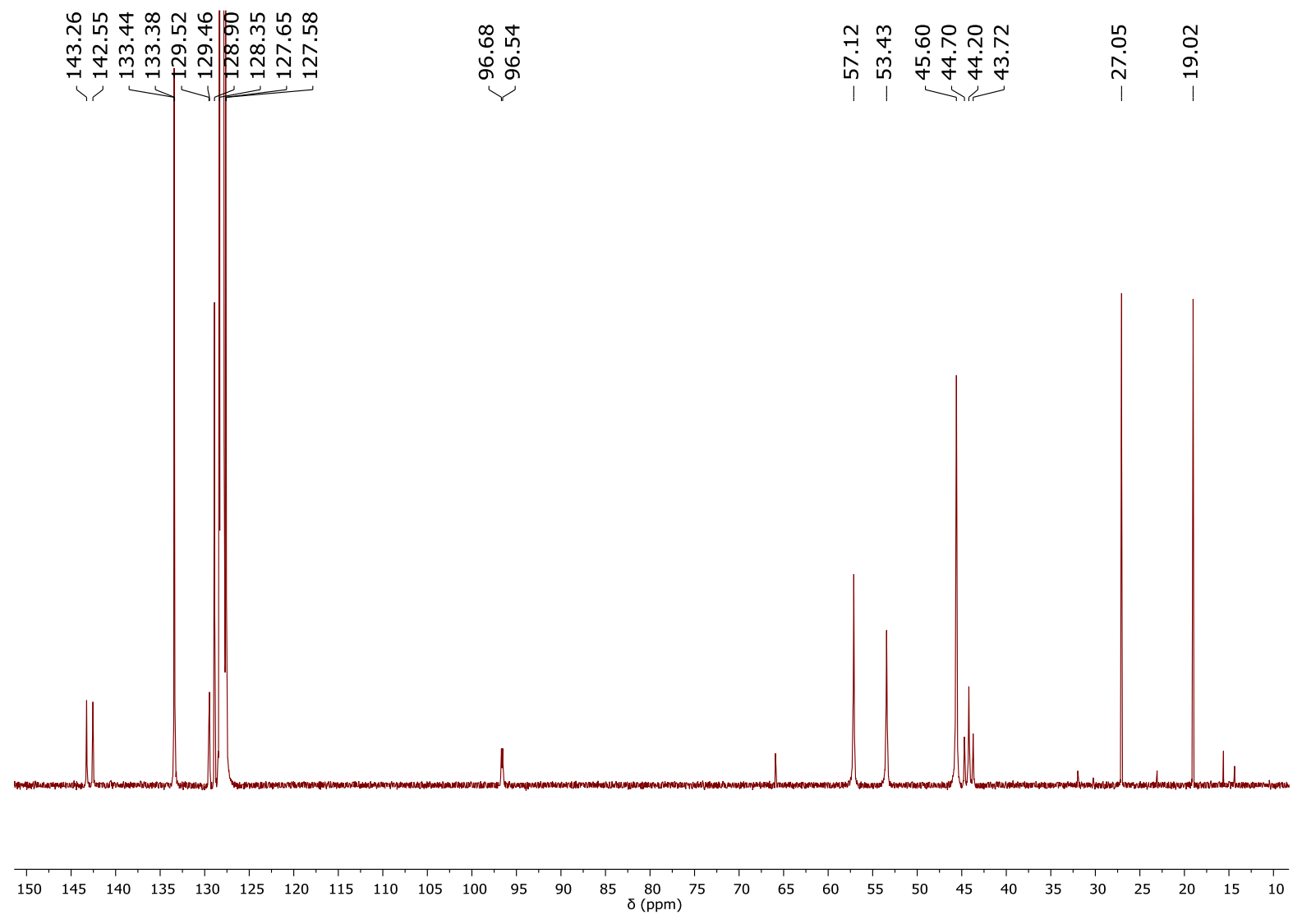

Figure S70. ${ }^{13} \mathrm{C}-\mathrm{NMR}\left(\mathrm{C}_{6} \mathrm{D}_{6}\right)$ of $\left[\mathrm{Ph}_{2} \mathrm{P}(\mathrm{O}) \mathrm{CHCHCMe}_{2} \mathrm{Li}(\mathrm{PMDETA})\right] \mathrm{8}$ 


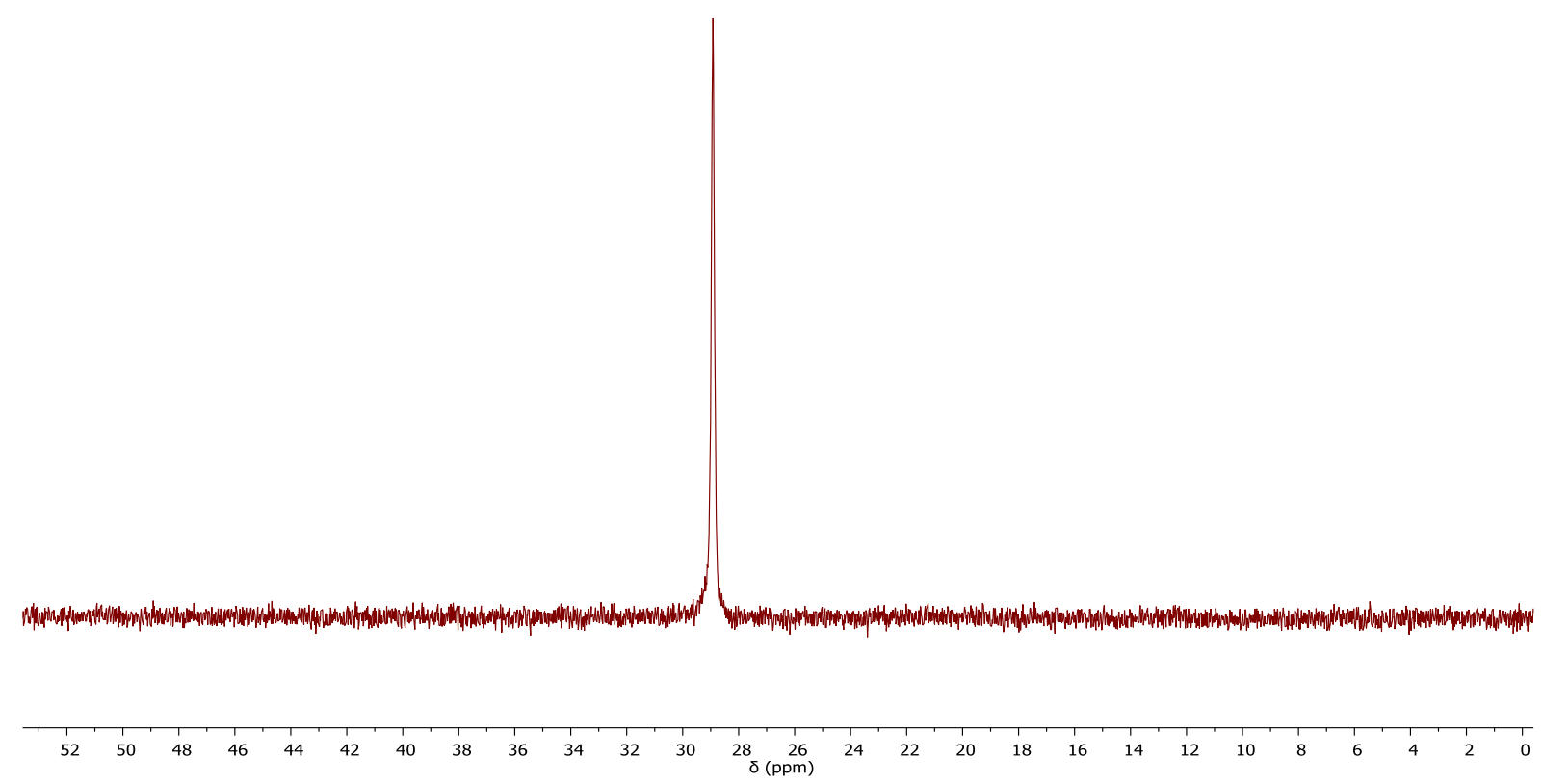

Figure S71. ${ }^{31} \mathrm{P}\{\mathrm{H}\}-\mathrm{NMR}\left(\mathrm{C}_{6} \mathrm{D}_{6}\right)$ of $\left[\mathrm{Ph}_{2} \mathrm{P}(\mathrm{O}) \mathrm{CHCHCMe}_{2} \mathrm{Li}(\mathrm{PMDETA})\right] \mathrm{8}$

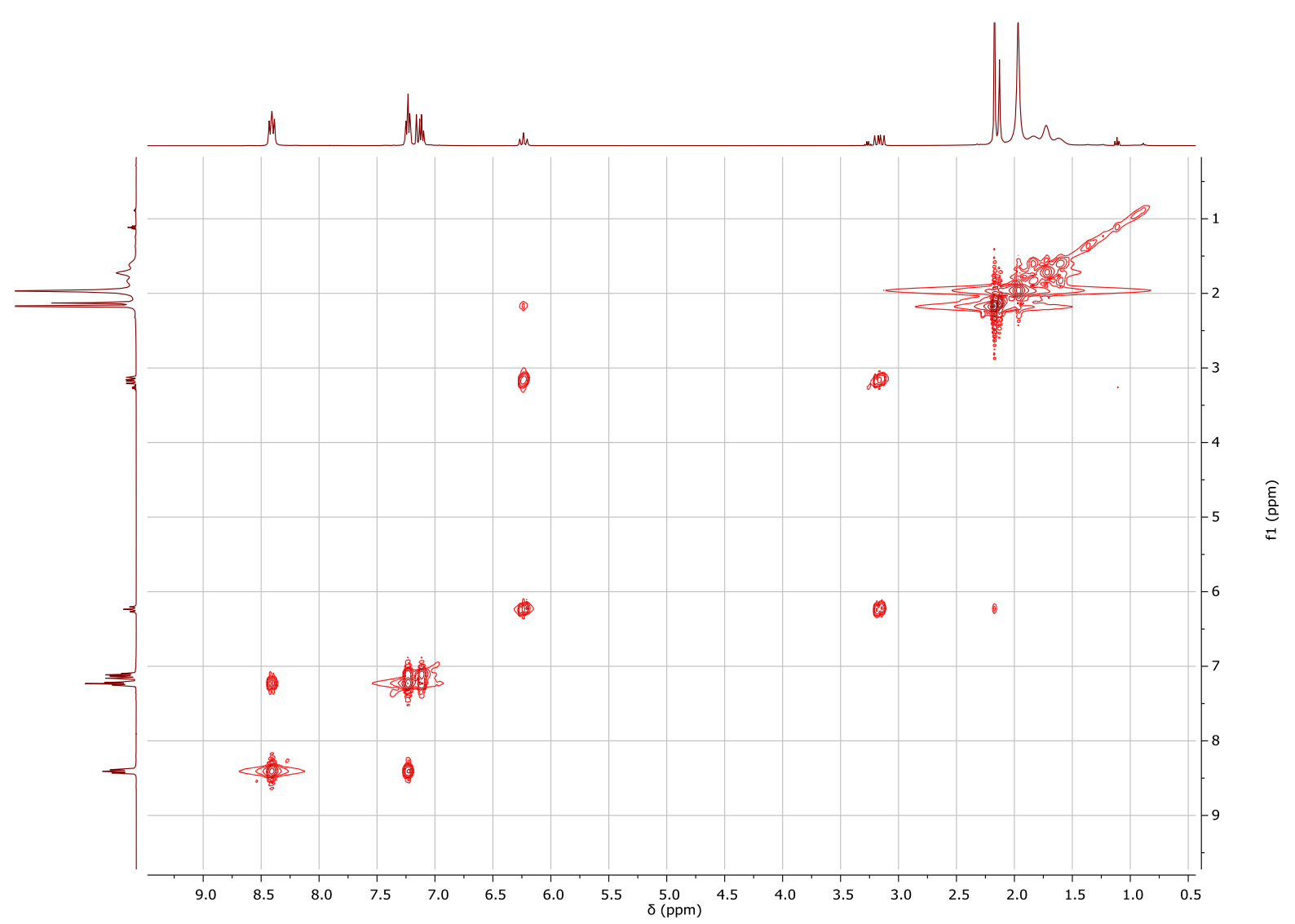

Figure S72. COSY-NMR $\left(\mathrm{C}_{6} \mathrm{D}_{6}\right)$ of $\left[\mathrm{Ph}_{2} \mathrm{P}(\mathrm{O}) \mathrm{CHCHCMe}_{2} \mathrm{Li}(\mathrm{PMDETA})\right] \textrm{ }$ 


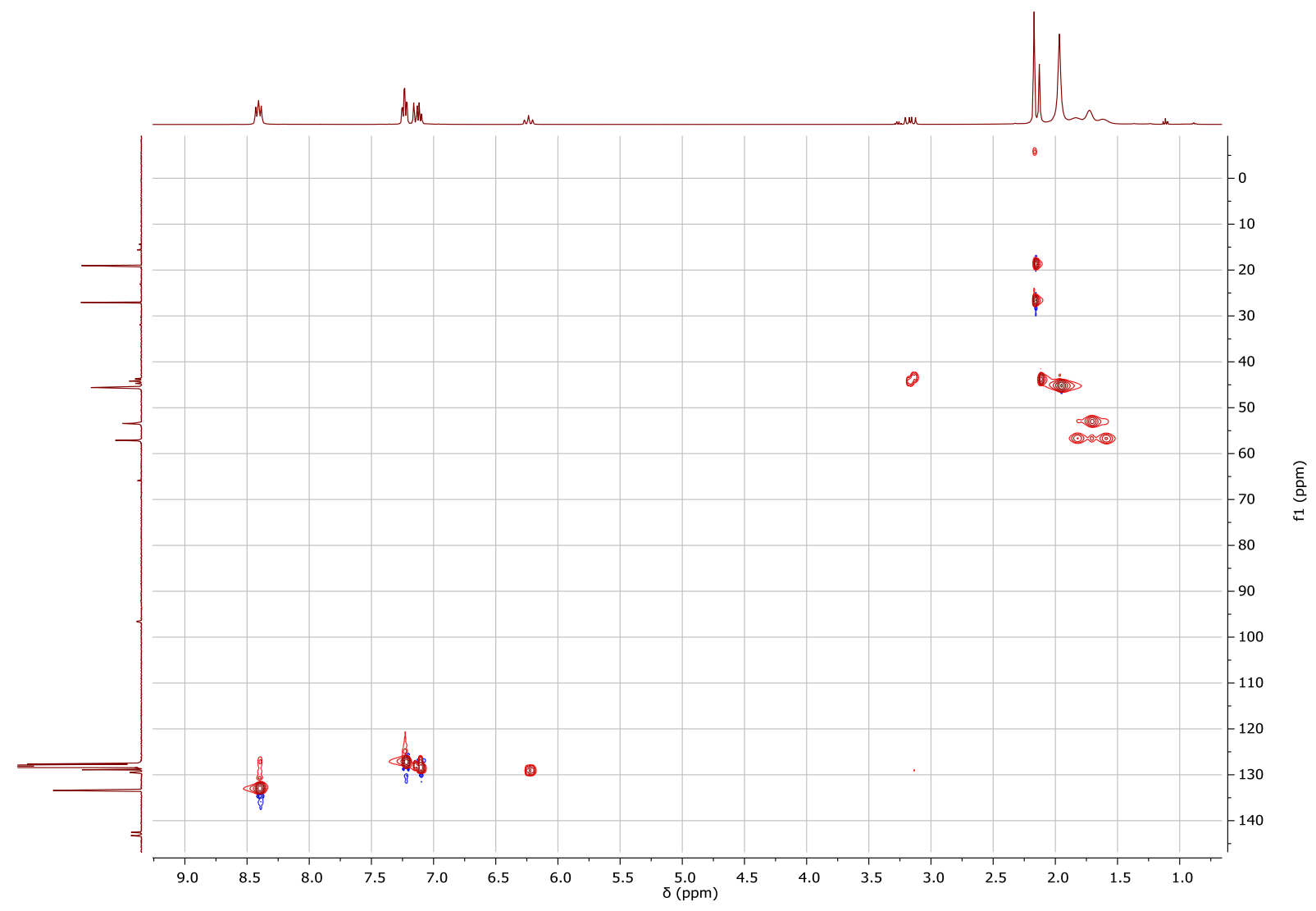

Figure S73. HMQC-NMR $\left(\mathrm{C}_{6} \mathrm{D}_{6}\right)$ of $\left[\mathrm{Ph}_{2} \mathrm{P}(\mathrm{O}) \mathrm{CHCHCMe}_{2} \mathrm{Li}(\mathrm{PMDETA})\right] \mathrm{8}$ 


\section{NMR spectra of $\left[\mathrm{Ph}_{2} \mathrm{P}(\mathrm{O}) \mathrm{CHCHCH}_{2} \mathrm{Li}(\mathrm{PMDETA})\right]$ 9:}

${ }^{1} \mathrm{H}$ NMR $\left(\mathrm{C}_{6} \mathrm{D}_{6}, 400.20 \mathrm{MHz}\right): \delta 1.74-2.37(\mathrm{~m}, 23 \mathrm{H}, \mathrm{PMDETA}), 3.70\left(\mathrm{dd},{ }^{2} J_{\mathrm{H}-\mathrm{P}}=21.6 \mathrm{~Hz},{ }^{3} J_{\mathrm{H}-\mathrm{H}(\beta)}=12.6 \mathrm{~Hz}, 1 \mathrm{H}, \mathrm{P}-\mathrm{CH}-\right)$ $4.21\left(\mathrm{dt},{ }^{3} J_{\mathrm{H}-\mathrm{H}(\beta)}=9.85 \mathrm{~Hz},{ }^{3} J_{\mathrm{H}-\mathrm{H}(\text { trans })}=3.7 \mathrm{~Hz}, 1 \mathrm{H},-\mathrm{CH}=\mathrm{CHH}\right), 4.63\left(\mathrm{dd},{ }^{3} J_{\mathrm{H}-\mathrm{H}(\beta)}=15.89 \mathrm{~Hz},{ }^{3} J_{\mathrm{H}-\mathrm{H}(\text { cis })}=3.3 \mathrm{~Hz}, 1 \mathrm{H},-\right.$ $\mathrm{CH}=\mathrm{CHH}), 6.88\left(\mathrm{tdd},{ }^{3} J_{\mathrm{H}-\mathrm{H}(\text { trans })}=15.89 \mathrm{~Hz},{ }^{3} J_{\mathrm{H}-\mathrm{H}(\alpha)}=12.63 \mathrm{~Hz},{ }^{3} J_{\mathrm{H}-\mathrm{H}(\text { cis })}=9.85 \mathrm{~Hz}, 1 \mathrm{H},-\mathrm{CH}=\mathrm{CH}_{2}\right), 7.11(\mathrm{~m}, 2 \mathrm{H}, p-\mathrm{H}), 7.23$ $(\mathrm{m}, 4 \mathrm{H}, m-\mathrm{H}), 8.36(\mathrm{~m}, 4 \mathrm{H}, o-\mathrm{H})$.

${ }^{7} \mathrm{Li}$ NMR (C6 6 6, $\left.155.53 \mathrm{MHz}\right): \delta-3.94$.

${ }^{13} \mathrm{C}$ NMR (C $\left.6 \mathrm{D}_{6}, 100.6 \mathrm{MHz}\right): \delta 44.05$ (br s, PMDETA), 45.77 (s, PMDETA), 51.22 (d, $\left.{ }^{1} J_{\mathrm{C}-\mathrm{P}}=146.5 \mathrm{~Hz}, \mathrm{P}-\mathrm{CH}-\right), 53.56$ (s, PMDETA), 57.38 (s, PMDETA), $81.58\left(\mathrm{~d},{ }^{3} J_{\mathrm{C}-\mathrm{P}}=21.8 \mathrm{~Hz},=\mathrm{CH}_{2}\right), 127.71\left(\mathrm{~d},{ }^{3} J_{\mathrm{C}-\mathrm{P}}=10.9 \mathrm{~Hz}, m-\mathrm{C}\right), 129.13\left(\mathrm{~d},{ }^{4} J_{\mathrm{C}-\mathrm{P}}=2.6\right.$ $\mathrm{Hz}, p-\mathrm{C}), 133.30\left(\mathrm{~d},{ }^{2} J_{\mathrm{C}-\mathrm{P}}=8.4 \mathrm{~Hz}, o-\mathrm{C}\right), 141.82\left(\mathrm{~d},{ }^{1} J_{\mathrm{C}-\mathrm{P}}=8.1 \mathrm{~Hz}, i-\mathrm{C}\right), 142.06\left(\mathrm{~d},{ }^{2} J_{\mathrm{C}-\mathrm{P}}=106.8 \mathrm{~Hz},-\mathrm{CH}=\right)$.

${ }^{31} \mathrm{P}\left\{{ }^{1} \mathrm{H}\right\} \mathrm{NMR}\left(\mathrm{C}_{6} \mathrm{D}_{6}, 162.0 \mathrm{MHz}\right): \delta 29.2$.

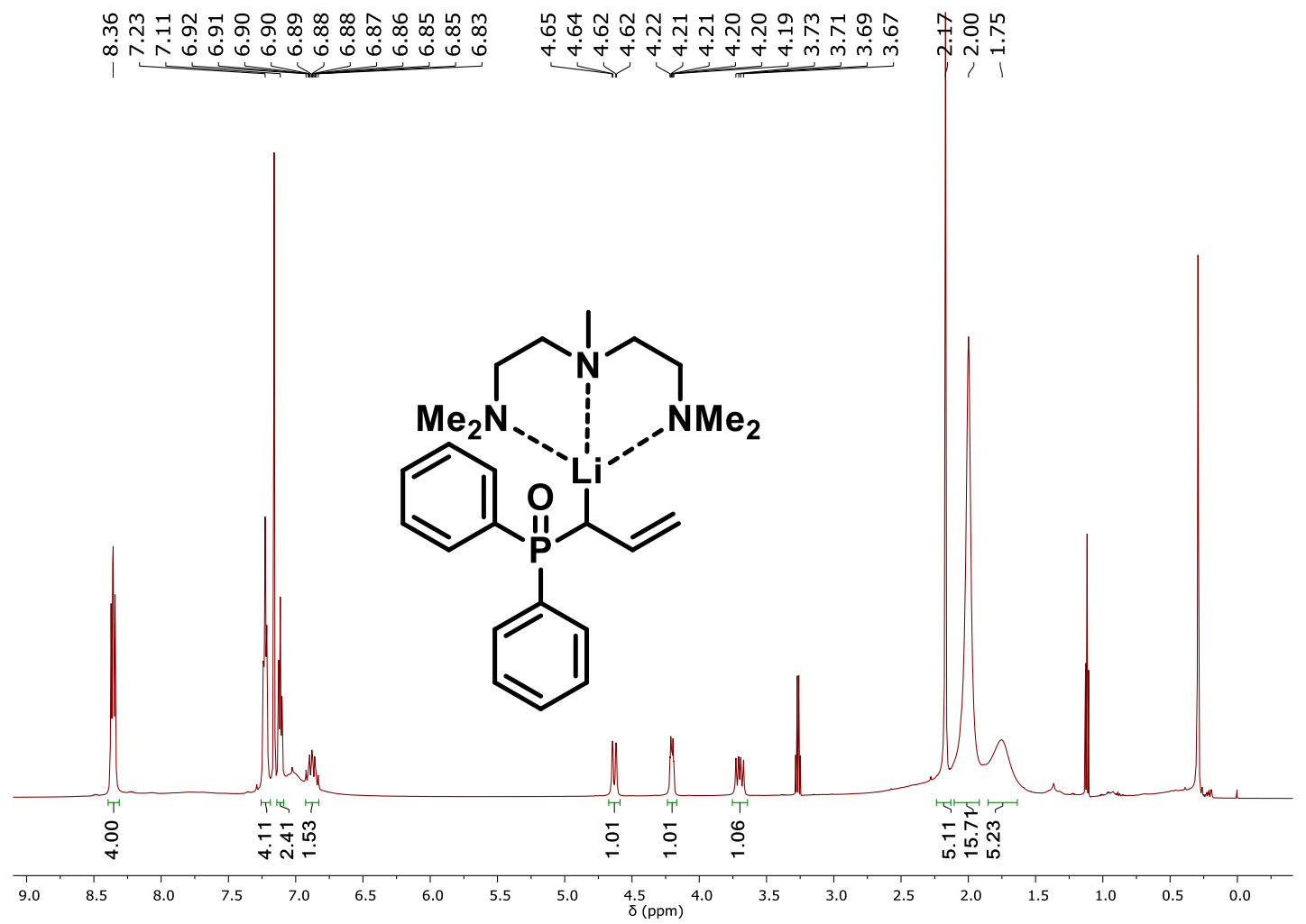

Figure S74. ${ }^{1} \mathrm{H}-\mathrm{NMR}\left(\mathrm{C}_{6} \mathrm{D}_{6}\right)$ of $\left[\mathrm{Ph}_{2} \mathrm{P}(\mathrm{O}) \mathrm{CHCHCH}_{2} \mathrm{Li}(\mathrm{PMDETA})\right] 9$ 


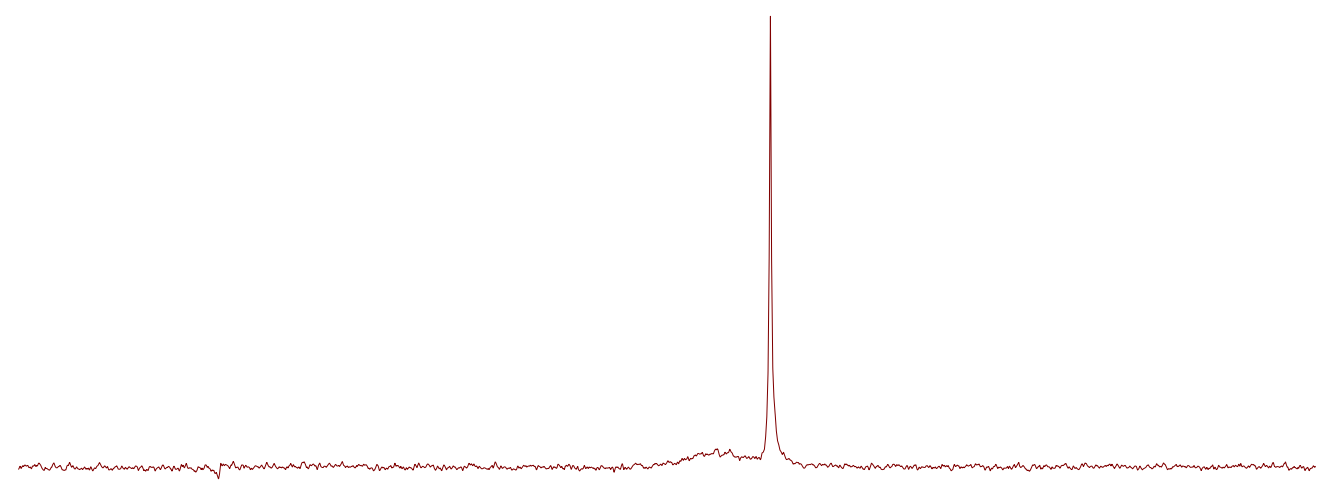

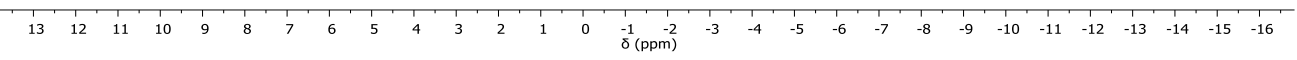

Figure S75. ${ }^{7} \mathrm{Li}-\mathrm{NMR}\left(\mathrm{C}_{6} \mathrm{D}_{6}\right)$ of $\left[\mathrm{Ph}_{2} \mathrm{P}(\mathrm{O}) \mathrm{CHCHCH}_{2} \mathrm{Li}(\mathrm{PMDETA})\right] 9$
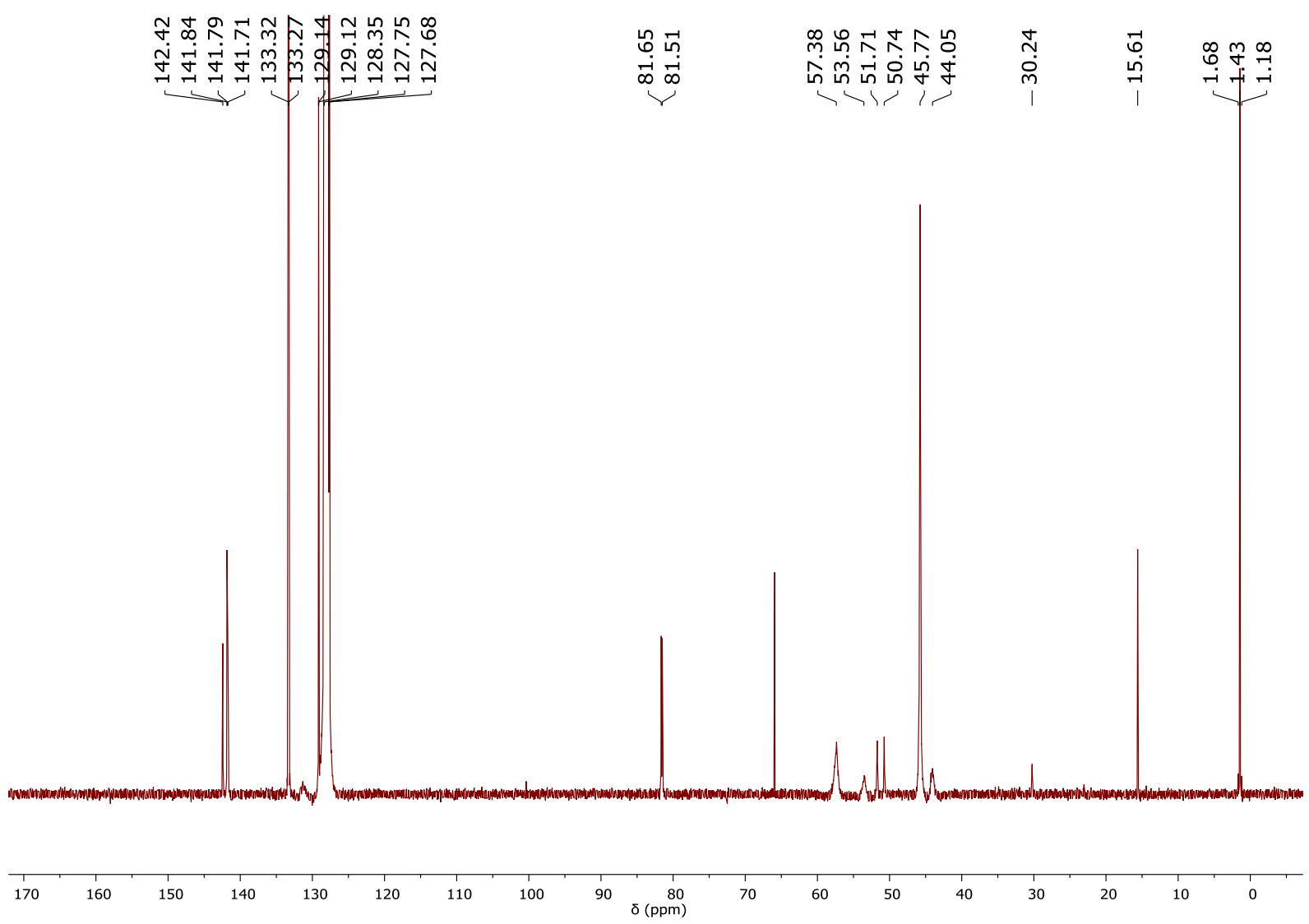

Figure S76. ${ }^{13} \mathrm{C}-\mathrm{NMR}\left(\mathrm{C}_{6} \mathrm{D}_{6}\right)$ of $\left[\mathrm{Ph}_{2} \mathrm{P}(\mathrm{O}) \mathrm{CHCHCH}_{2} \mathrm{Li}(\mathrm{PMDETA})\right] 9$ 


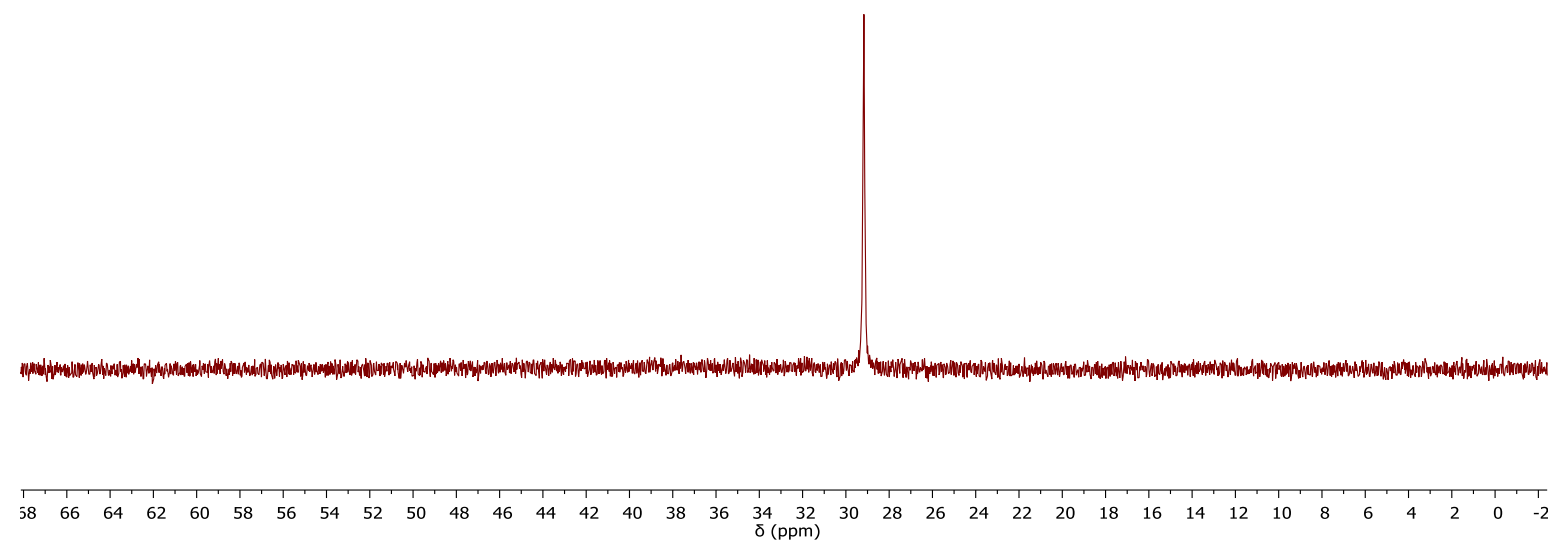

Figure S77. ${ }^{31} \mathrm{P}\left\{{ }^{1} \mathrm{H}\right\}-\mathrm{NMR}\left(\mathrm{C}_{6} \mathrm{D}_{6}\right)$ of $\left[\mathrm{Ph}_{2} \mathrm{P}(\mathrm{O}) \mathrm{CHCHCH}_{2} \mathrm{Li}(\mathrm{PMDETA})\right] 9$

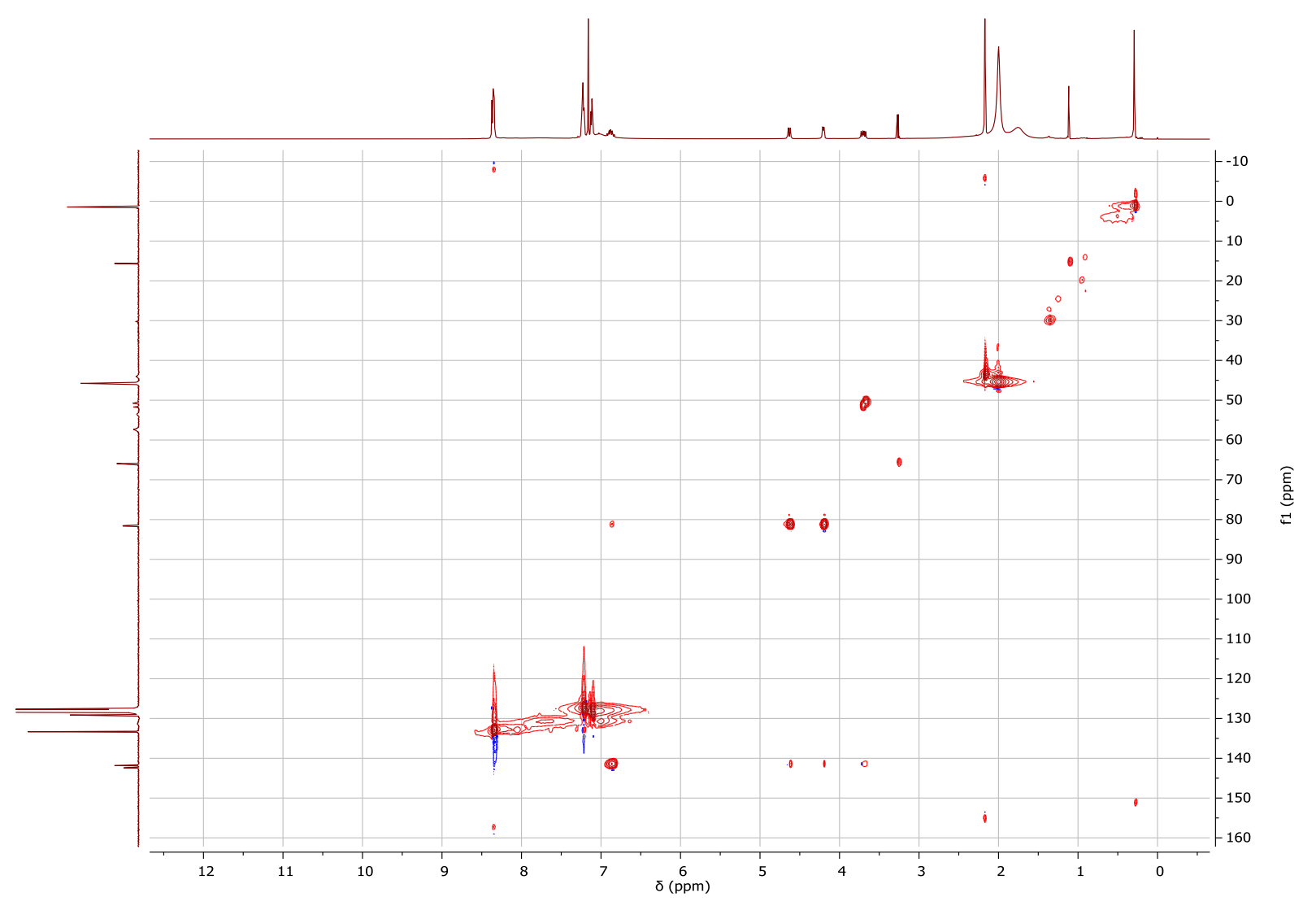

Figure S78. HMQC-NMR $\left(\mathrm{C}_{6} \mathrm{D}_{6}\right)$ of $\left[\mathrm{Ph}_{2} \mathrm{P}(\mathrm{O}) \mathrm{CHCHCH}_{2} \mathrm{Li}(\mathrm{PMDETA})\right] 9$ 


\section{NMR spectra of $\left[\mathrm{Ph}_{2} \mathrm{PCHCHCMe} 2 \mathrm{Li}(\mathrm{PMDETA})\right]$ 10:}

${ }^{1} \mathrm{H}$ NMR $\left(\mathrm{C}_{6} \mathrm{D}_{6}, 400.20 \mathrm{MHz}\right): \delta 1.50-1.85(\mathrm{~m}, 23 \mathrm{H}, \mathrm{PMDETA}) 2.07\left(\mathrm{~m}, 6 \mathrm{H},=\mathrm{CMe}_{2}\right), 2.63\left(\mathrm{dd},{ }^{3} J_{\mathrm{H}-\mathrm{H}}=13.0 \mathrm{~Hz},{ }^{2} J_{\mathrm{H}-\mathrm{P}}=7.5\right.$ $\mathrm{Hz}, 1 \mathrm{H}, \mathrm{P}-\mathrm{CH}-), 6.37\left(\mathrm{t},{ }^{3} J_{\mathrm{H}-\mathrm{H}}=13.0 \mathrm{~Hz},{ }^{3} J_{\mathrm{H}-\mathrm{P}}=12.5 \mathrm{~Hz}, 1 \mathrm{H},-\mathrm{CH}=\right), 7.06(\mathrm{~m}, 2 \mathrm{H}, p-\mathrm{H}), 7.20(\mathrm{~m}, 4 \mathrm{H}, m-\mathrm{H}), 7.87(\mathrm{~m}, 4 \mathrm{H}$, $o-\mathrm{H})$.

${ }^{7} \mathrm{Li}$ NMR $\left(\mathrm{C}_{6} \mathrm{D}_{6}, 155.53 \mathrm{MHz}\right): \delta-4.50$.

${ }^{13} \mathrm{C}$ NMR $\left(\mathrm{C}_{6} \mathrm{D}_{6}, 100.6 \mathrm{MHz}\right): \delta 19.71\left(\mathrm{~d},{ }^{4} J_{\mathrm{C}-\mathrm{P}}=3.1 \mathrm{~Hz},=\mathrm{CMeMe}(\operatorname{trans})\right), 27.39\left(\mathrm{~d},{ }^{4} J_{\mathrm{C}-\mathrm{P}}=2.6 \mathrm{~Hz},=\mathrm{CMeMe}(\mathrm{cis})\right), 37.24$ (br m, P-CH-), 44.55 (s, PMDETA), 45.86 (s, PMDETA), 53.48 (s, PMDETA), 57.22 (s, PMDETA), 95.94 (d, ${ }^{3} J_{\mathrm{C}-\mathrm{P}}=23.3$ $\left.\mathrm{Hz},=\mathrm{CMe}_{2}\right), 125.93(\mathrm{~s}, p-\mathrm{C}), 127.69\left(\mathrm{~d},{ }^{3} J_{\mathrm{C}-\mathrm{P}}=5.3 \mathrm{~Hz}, m-\mathrm{C}\right), 132.87\left(\mathrm{~d},{ }^{2} J_{\mathrm{C}-\mathrm{P}}=16.9 \mathrm{~Hz}, o-\mathrm{C}\right), 136.79\left(\mathrm{~d},{ }^{2} J_{\mathrm{C}-\mathrm{P}}=28.1 \mathrm{~Hz},-\right.$ $\mathrm{CH}=), 151.88\left(\mathrm{~d},{ }^{1} J_{\mathrm{C}-\mathrm{P}}=19.7 \mathrm{~Hz}, i-\mathrm{C}\right)$.

${ }^{31} \mathrm{P}\left\{{ }^{1} \mathrm{H}\right\}$ NMR $\left(\mathrm{C}_{6} \mathrm{D}_{6}, 162.00 \mathrm{MHz}\right): \delta-2.5$.

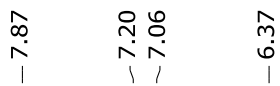

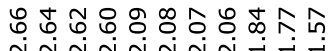

1

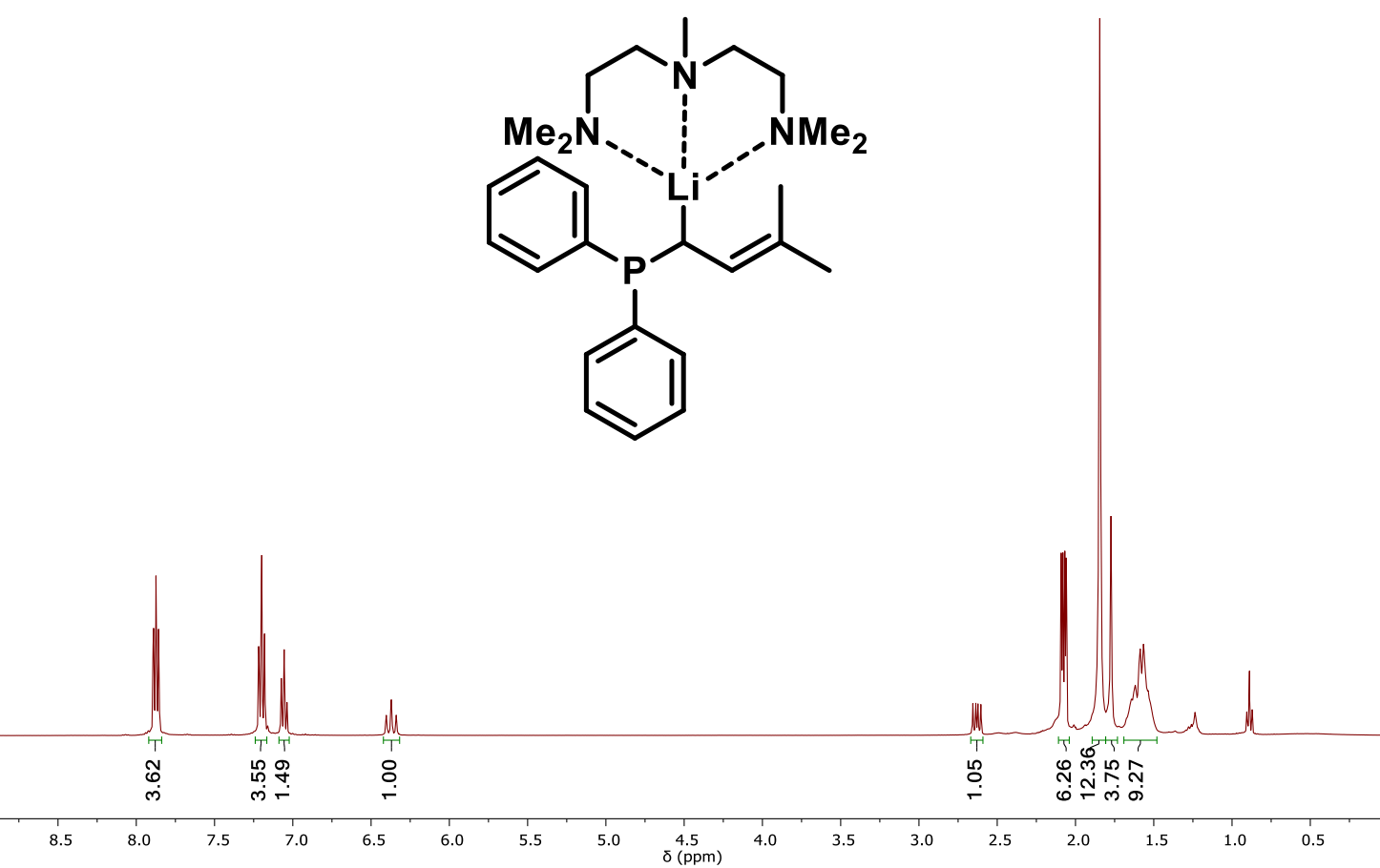
iñ

Figure S79. ${ }^{1} \mathrm{H}-\mathrm{NMR}\left(\mathrm{C}_{6} \mathrm{D}_{6}\right)$ of $\left[\mathrm{Ph}_{2} \mathrm{PCHCHCMe} 2 \mathrm{Li}(\mathrm{PMDETA})\right] \mathbf{1 0}$ 


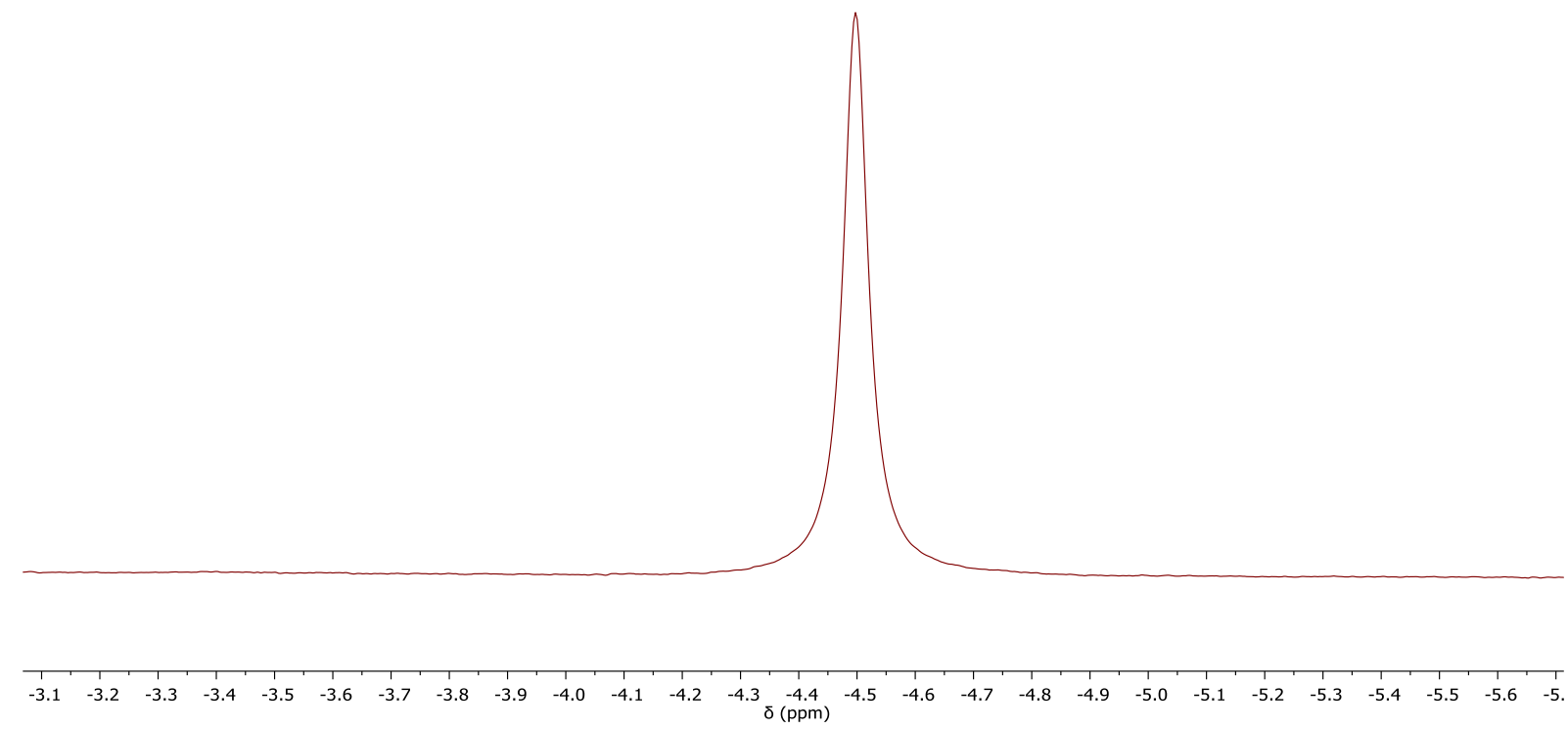

Figure S80. ${ }^{7} \mathrm{Li}-\mathrm{NMR}\left(\mathrm{C}_{6} \mathrm{D}_{6}\right)$ of $\left[\mathrm{Ph}_{2} \mathrm{PCHCHCMe}_{2} \mathrm{Li}(\mathrm{PMDETA})\right] \mathbf{1 0}$

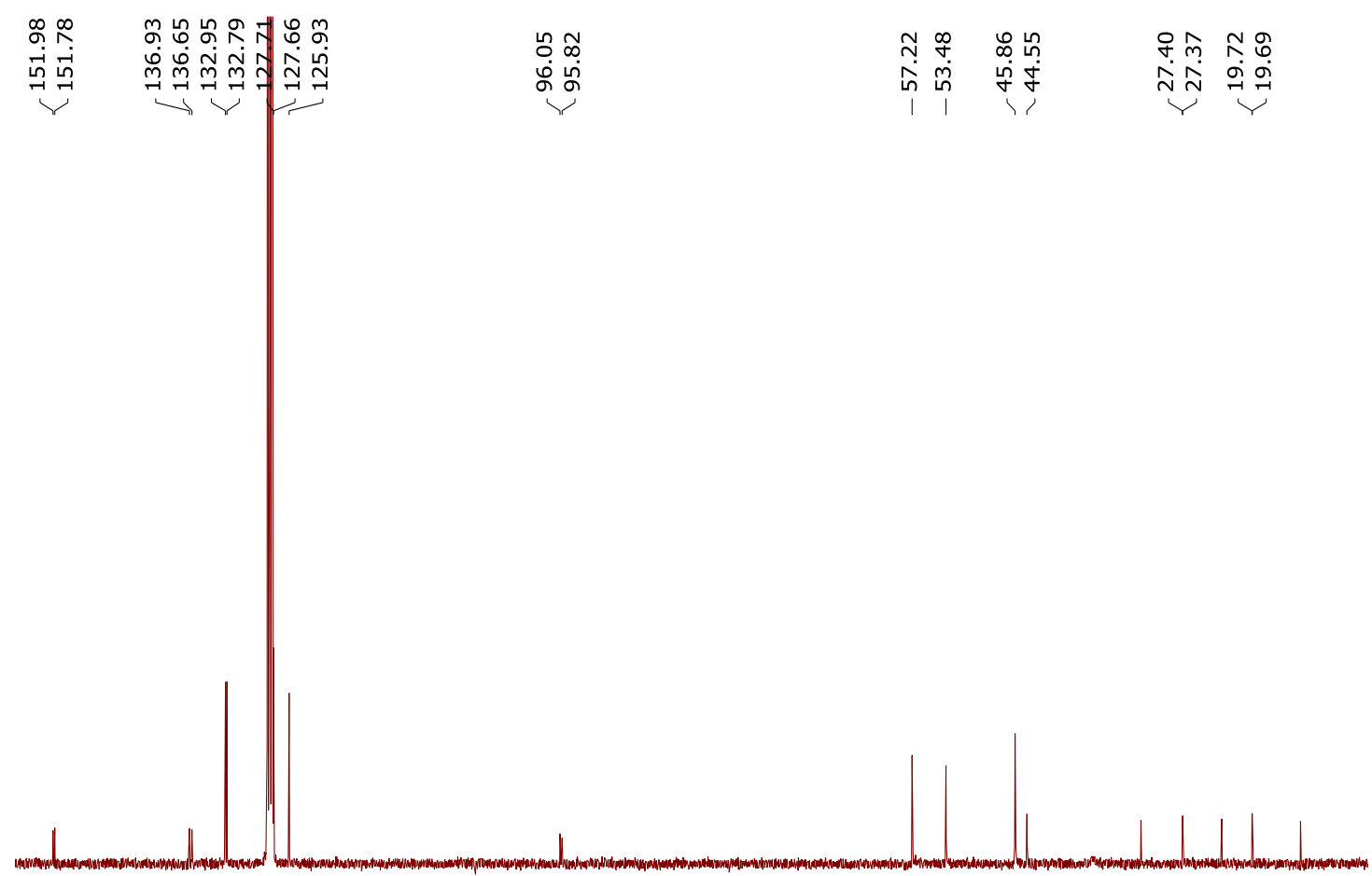

\begin{tabular}{llllllllllllllllllllllllllllllllll}
155 & 150 & 145 & 140 & 135 & 130 & 125 & 120 & 115 & 110 & 105 & 100 & 95 & 90 & 85 & 80 & 75 & 70 & 65 & 60 & 55 & 50 & 45 & 40 & 35 & 30 & 25 & 20 & 15 & 10 \\
\hline$(\mathrm{ppm})$ & & & &
\end{tabular}

Figure S81. ${ }^{13} \mathrm{C}-\mathrm{NMR}\left(\mathrm{C}_{6} \mathrm{D}_{6}\right)$ of $\left[\mathrm{Ph}_{2} \mathrm{PCHCHCMe} 2 \mathrm{Li}(\mathrm{PMDETA})\right] \mathbf{1 0}$ 


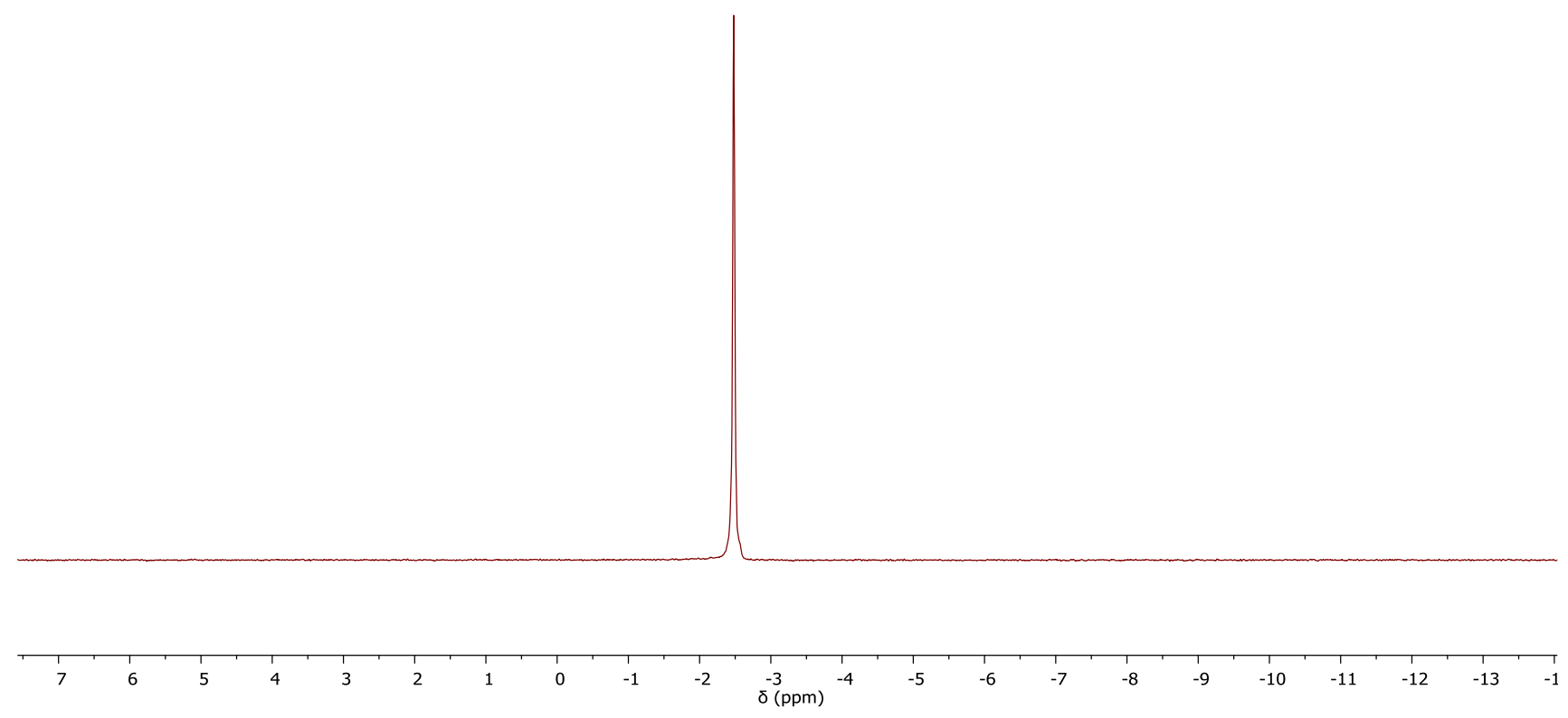

Figure S82. ${ }^{31} \mathrm{P}\{\mathrm{H}\}-\mathrm{NMR}\left(\mathrm{C}_{6} \mathrm{D}_{6}\right)$ of $\left[\mathrm{Ph}_{2} \mathrm{PCHCHCMe} 2 \mathrm{Li}(\mathrm{PMDETA})\right] 10$

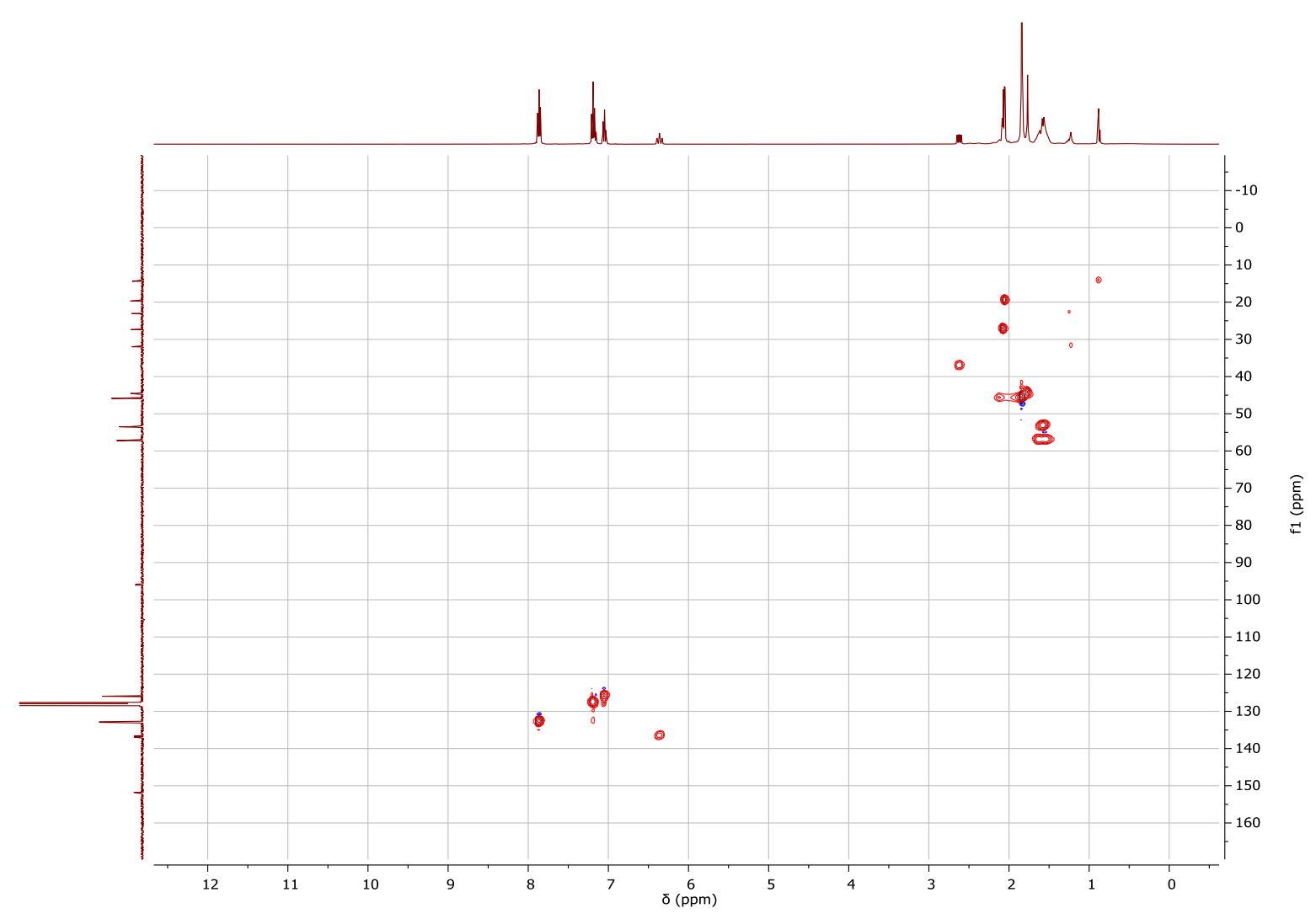

Figure S83. HMQC-NMR $\left(\mathrm{C}_{6} \mathrm{D}_{6}\right)$ of $\left[\mathrm{Ph}_{2} \mathrm{PCHCHCMe}_{2} \mathrm{Li}(\mathrm{PMDETA})\right] 10$ 


\section{NMR spectra of [Ph 2 PCHC(Me) $\left.\mathrm{CH}_{2} \mathrm{Li}(\mathrm{TMEDA})\right]$ 11:}

${ }^{1} \mathrm{H}$ NMR $\left(\mathrm{C}_{6} \mathrm{D}_{6}, 400.20 \mathrm{MHz}\right): \delta 1.56$ (br-s, 4H, TMEDA-CH$), 1.73$ (br-s, 12H, TMEDA-CH 3 ), 2.34 (br-s, 3H, -C(Me)=), 3.27 (br-s, 1H, =CHH), 3.34 (br-s, 1H =CHH), 3.55 (br-s, 1H, P-CH-), 7.08 (m, 2H, p-H), 7.20 (m, 4H, m-H), 7.82 (m, 4H, $o-\mathrm{H})$.

${ }^{1} \mathrm{H}$ NMR (THF-d8, $400.20 \mathrm{MHz}$ ): $\delta 1.93$ (s, 3H, -C(Me)=), 2.15 (s, 12H, TMEDA-CH 3 ), 2.31 (s, 4H, TMEDA-CH2), 2.73 (br-s, 1H, =CHH), 2.97 (br-s, 1H, P-CH-), 3.18 (br-s, 1H =CHH), 7.00 (m, 2H, p-H), 7.09 (m, 4H, $m-\mathrm{H}), 7.36(\mathrm{~m}, 4 \mathrm{H}, o-\mathrm{H})$.

${ }^{7} \mathrm{Li} \mathrm{NMR}\left(\mathrm{C}_{6} \mathrm{D}_{6}, 155.53 \mathrm{MHz}\right): \delta-4.47$.

${ }^{7} \mathrm{Li}$ NMR (THF-d8, $\left.155.53 \mathrm{MHz}\right): \delta-6.82$.

${ }^{13} \mathrm{C}$ NMR $\left(\mathrm{C}_{6} \mathrm{D}_{6}, 100.6 \mathrm{MHz}\right): \delta 23.06$ (br-m, $\left.-\mathrm{C}\left(\mathrm{CH}_{3}\right)=\right)$, 45.71 (s, TMEDA-CH 3 ), 55.02 (br-m, = $\left.\mathrm{CH}_{2}\right), 56.45$ (s, TMEDA$\mathrm{CH}_{2}$ ), 67.37 (br-m, P-CH-), 126.52 (br-s, $p$-C), 128.00 (m, $m$-C), 132.66 (br-s, $\left.o-\mathrm{C}\right), 150.01$ (br-m, -C(Me)=), 157.43 (br-m, $i$-C).

${ }^{13} \mathrm{C}$ NMR (THF-d8, 100.6 MHz): $\delta 24.55$ (br-m, -C(CH3)=), 46.31 (s, TMEDA-CH $\left.\mathrm{CH}_{3}\right), 56.53$ (br-m, P-CH-), 58.91 (s, TMEDA-CH 2$), 67.13$ (br-m, =CH2), $126.21(\mathrm{~s}, p-\mathrm{C}), 127.86\left(\mathrm{~d},{ }^{3} J_{\mathrm{C}-\mathrm{P}}=5.6 \mathrm{~Hz}, m-\mathrm{C}\right), 132.82\left(\mathrm{~d},{ }^{2} J_{\mathrm{C}-\mathrm{P}}=18.0 \mathrm{~Hz}, o-\mathrm{C}\right)$, 150.16 (br-m, $i$-C), 158.24 (d, ${ }^{2} J_{\mathrm{C}-\mathrm{P}}=29.1 \mathrm{~Hz},-\mathrm{C}(\mathrm{Me})=$ ).

${ }^{31} \mathrm{P}\left\{{ }^{1} \mathrm{H}\right\}$ NMR $\left(\mathrm{C}_{6} \mathrm{D}_{6}, 162.00 \mathrm{MHz}\right): \delta-32.7,-27.6,-19.7$.

${ }^{31} \mathrm{P}\left\{{ }^{1} \mathrm{H}\right\}$ NMR (THF-d $\left.8,162.00 \mathrm{MHz}\right): \delta-17.4$.

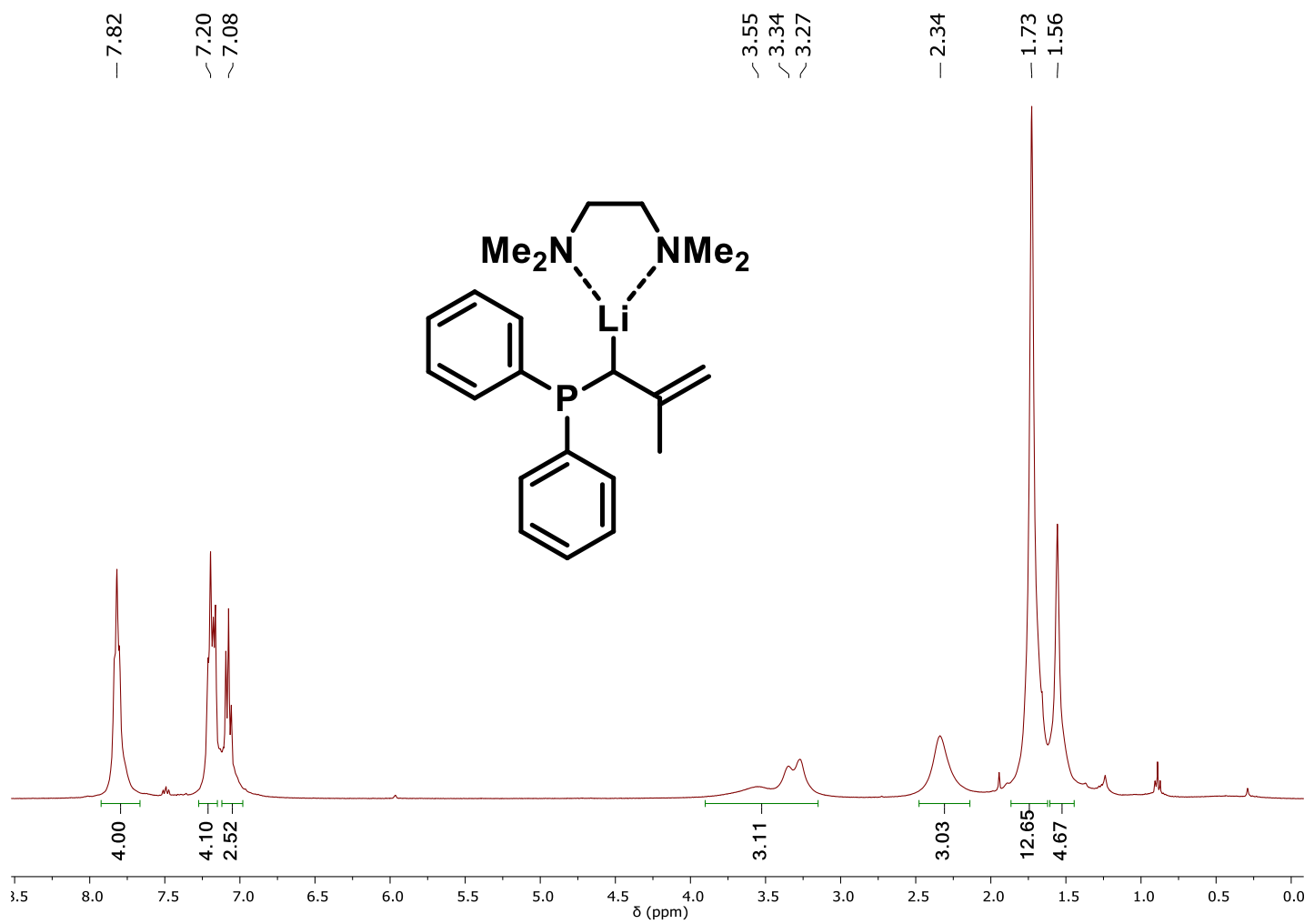

Figure S84. ${ }^{1} \mathrm{H}-\mathrm{NMR}\left(\mathrm{C}_{6} \mathrm{D}_{6}\right)$ of $\left[\mathrm{Ph}_{2} \mathrm{PCHC}(\mathrm{Me}) \mathrm{CH}_{2} \mathrm{Li}(\mathrm{TMEDA})\right] 11$ 


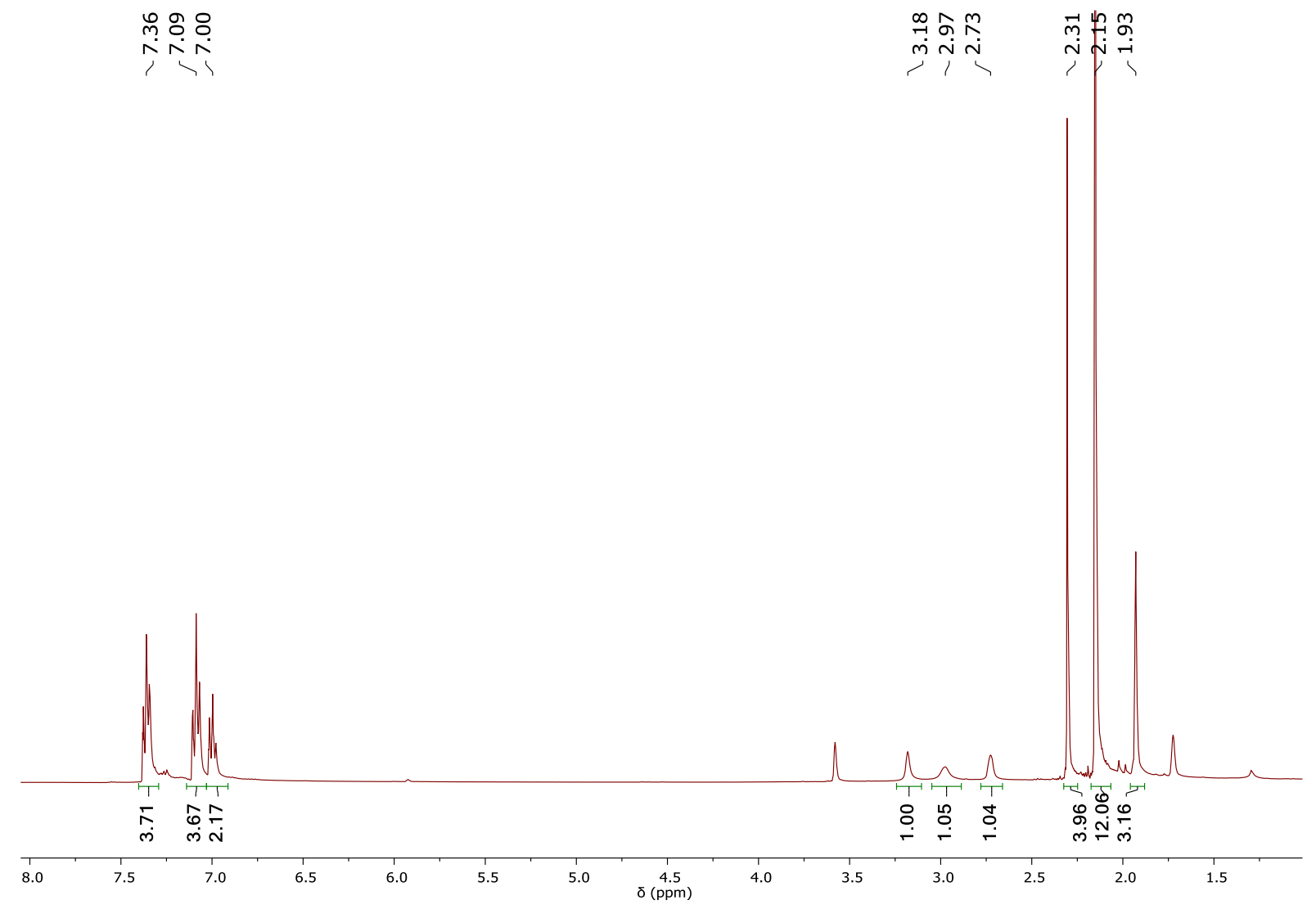

Figure S85. ${ }^{1} \mathrm{H}-\mathrm{NMR}$ (THF-d 8 ) of $\left[\mathrm{Ph}_{2} \mathrm{PCHC}(\mathrm{Me}) \mathrm{CH}_{2} \mathrm{Li}(\mathrm{TMEDA})\right] \mathbf{1 1}$

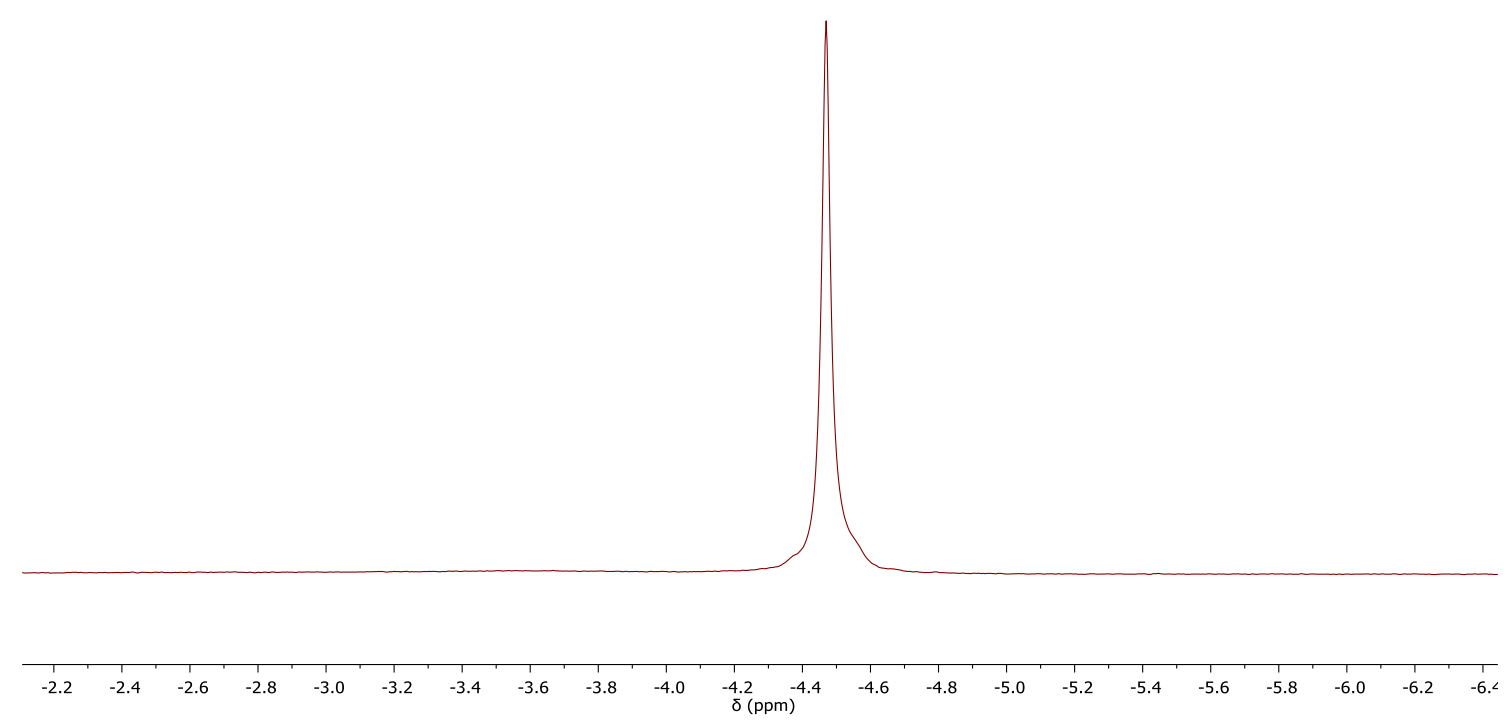

Figure S86. ${ }^{7} \mathrm{Li}-\mathrm{NMR}\left(\mathrm{C}_{6} \mathrm{D}_{6}\right)$ of $\left[\mathrm{Ph}_{2} \mathrm{PCHC}(\mathrm{Me}) \mathrm{CH}_{2} \mathrm{Li}(\mathrm{TMEDA})\right] 11$ 


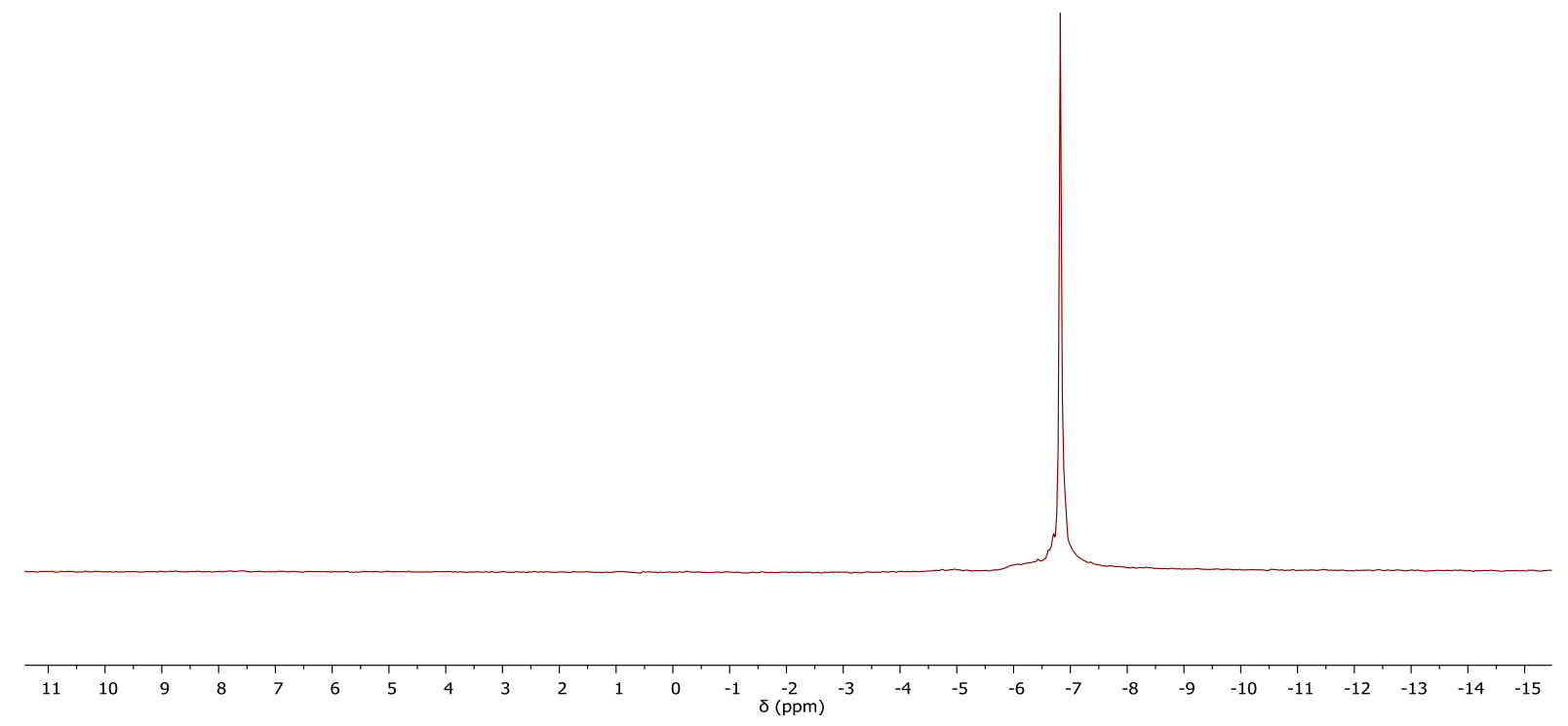

Figure S87. ${ }^{7} \mathrm{Li}-\mathrm{NMR}$ (THF-d $\mathrm{d}_{8}$ ) of $\left[\mathrm{Ph}_{2} \mathrm{PCHC}^{\mathrm{PC}} \mathrm{Me} \mathrm{CH}_{2} \mathrm{Li}\right.$ (TMEDA) $] 11$
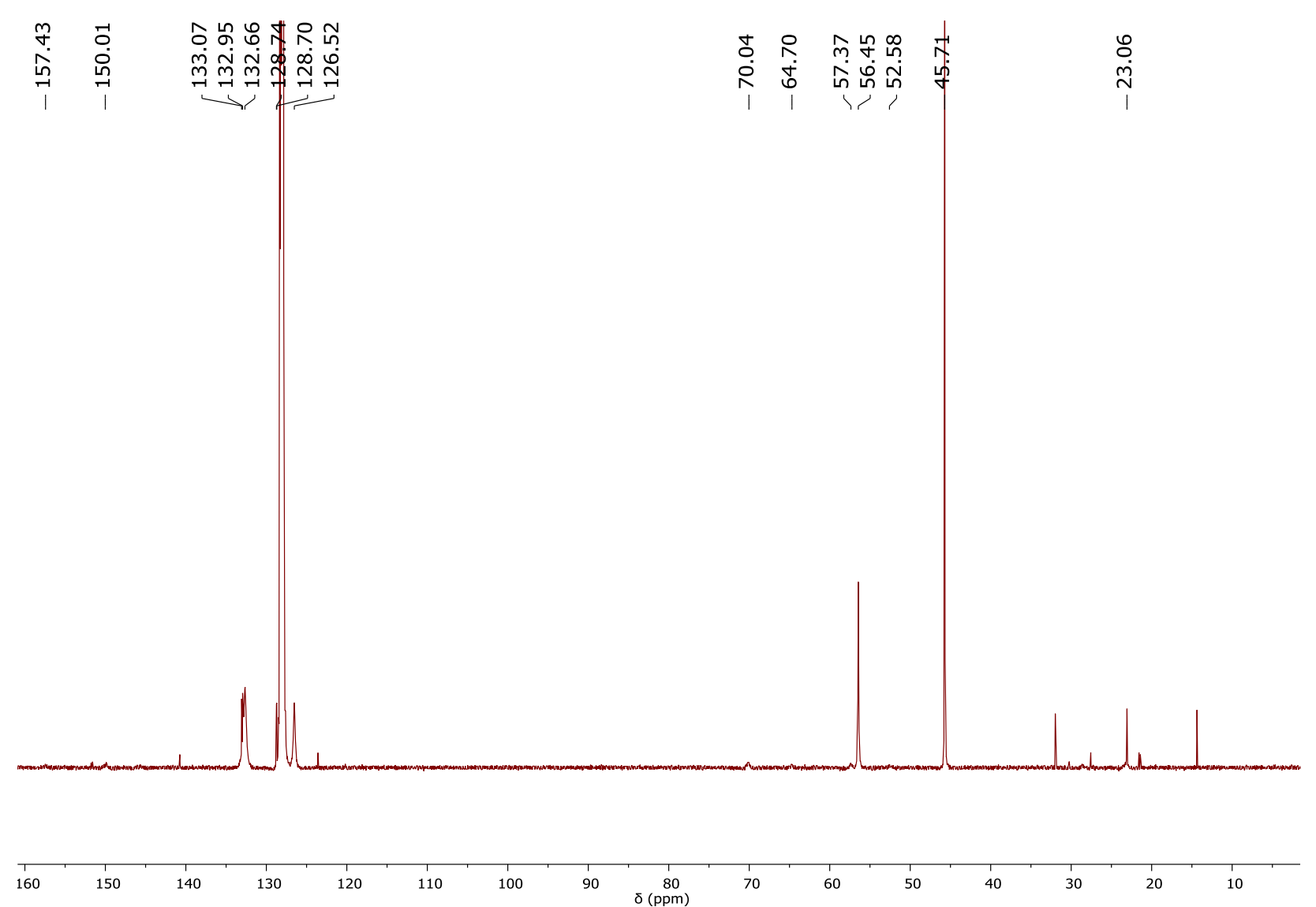

Figure S88. ${ }^{13} \mathrm{C}-\mathrm{NMR}\left(\mathrm{C}_{6} \mathrm{D}_{6}\right)$ of $\left[\mathrm{Ph}_{2} \mathrm{PCHC}(\mathrm{Me}) \mathrm{CH}_{2} \mathrm{Li}(\mathrm{TMEDA})\right] \mathbf{1 1}$ 


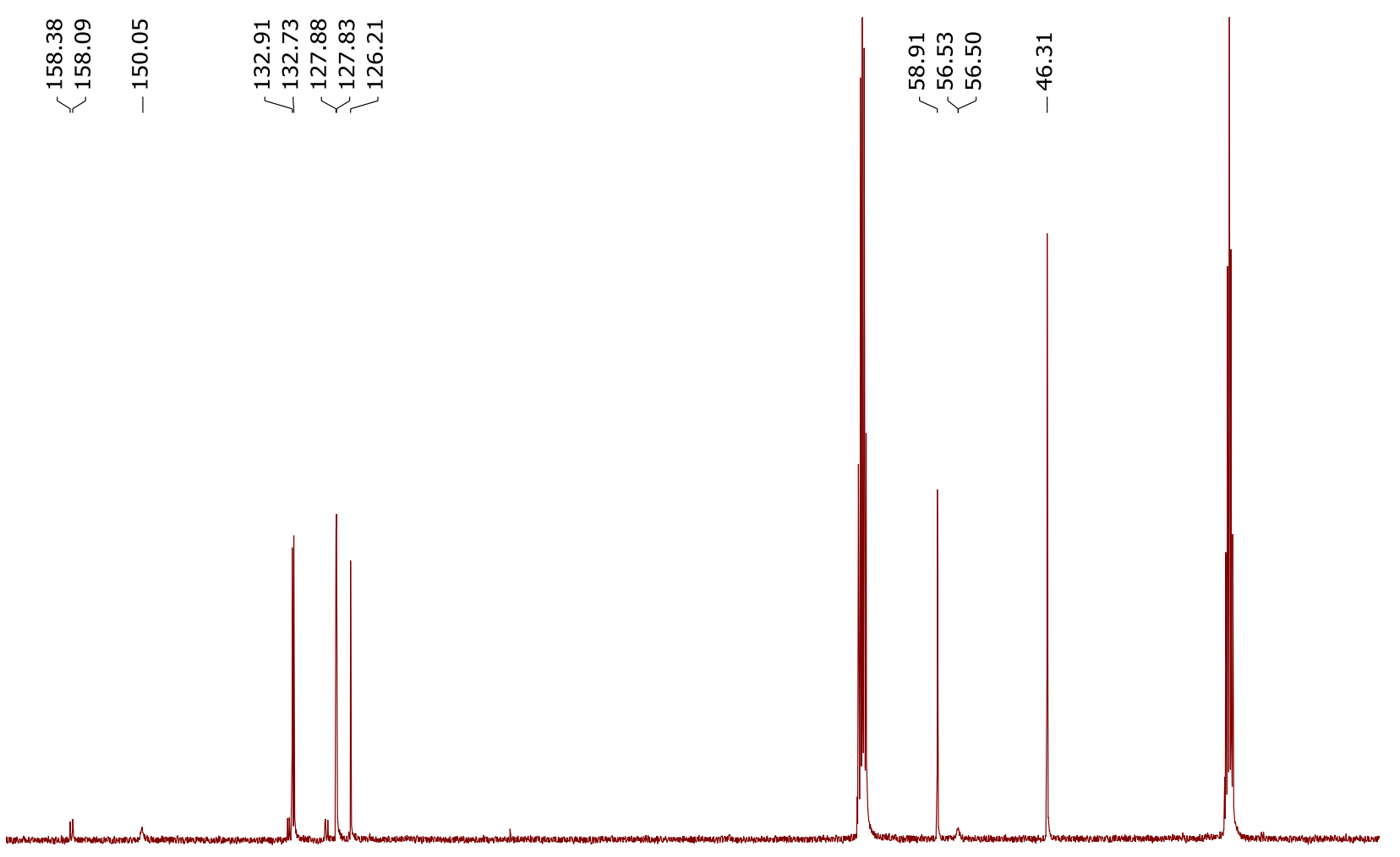

\begin{tabular}{lllllllllllllll}
1 & 1 & 1 & 1 & 1 & 1 & 1 & 1 & 1 & 1 & 1 & 1 & 1 \\
\hline
\end{tabular}

Figure S89. ${ }^{13} \mathrm{C}-\mathrm{NMR}(\mathrm{THF}-\mathrm{d} 8)$ of $\left[\mathrm{Ph}_{2} \mathrm{PCHC}(\mathrm{Me}) \mathrm{CH}_{2} \mathrm{Li}(\mathrm{TMEDA})\right] \mathbf{1 1}$

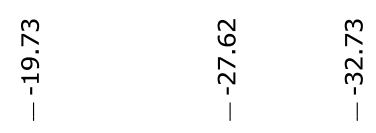

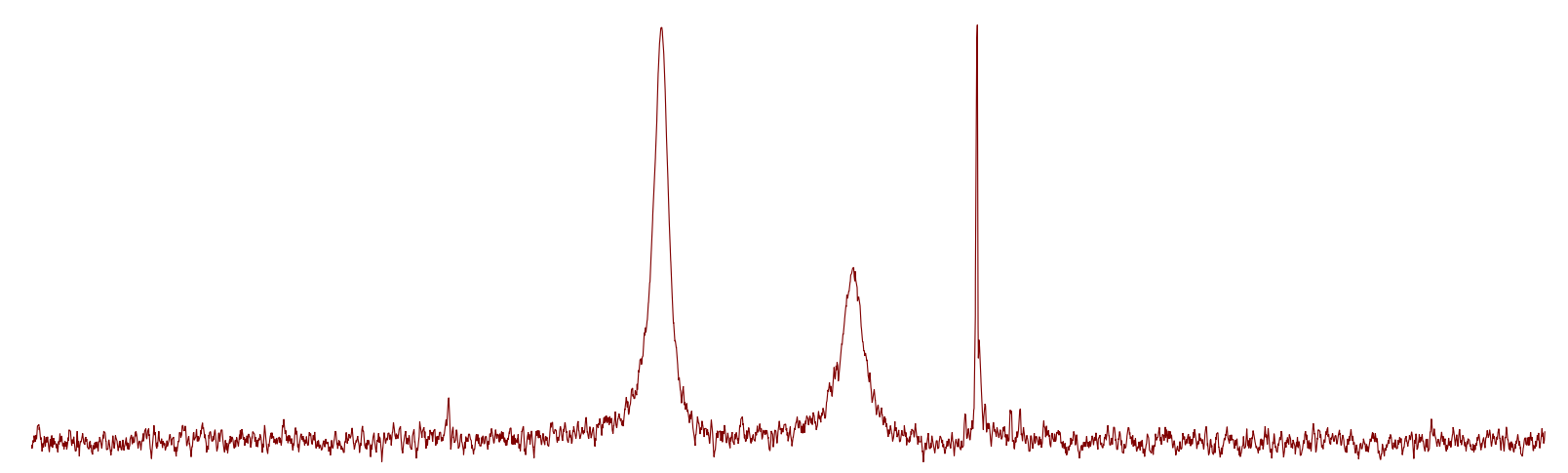

\begin{tabular}{llllllllllllllllllllllllllllllllllll}
\hline 6 & 4 & 2 & 0 & -2 & -4 & -6 & -8 & -10 & -12 & -14 & -16 & -18 & -20 & -22 & -24 & -26 & -28 & -30 & -32 & -34 & -36 & -38 & -40 & -42 & -44 & -46 & -48 & -50 & -52 & -54 & -5
\end{tabular}

Figure S90. ${ }^{31} \mathrm{P}\left\{{ }^{1} \mathrm{H}\right\}-\mathrm{NMR}\left(\mathrm{C}_{6} \mathrm{D}_{6}\right)$ of $\left[\mathrm{Ph}_{2} \mathrm{PCHC}(\mathrm{Me}) \mathrm{CH}_{2} \mathrm{Li}(\mathrm{TMEDA})\right] \mathbf{1 1}$ 


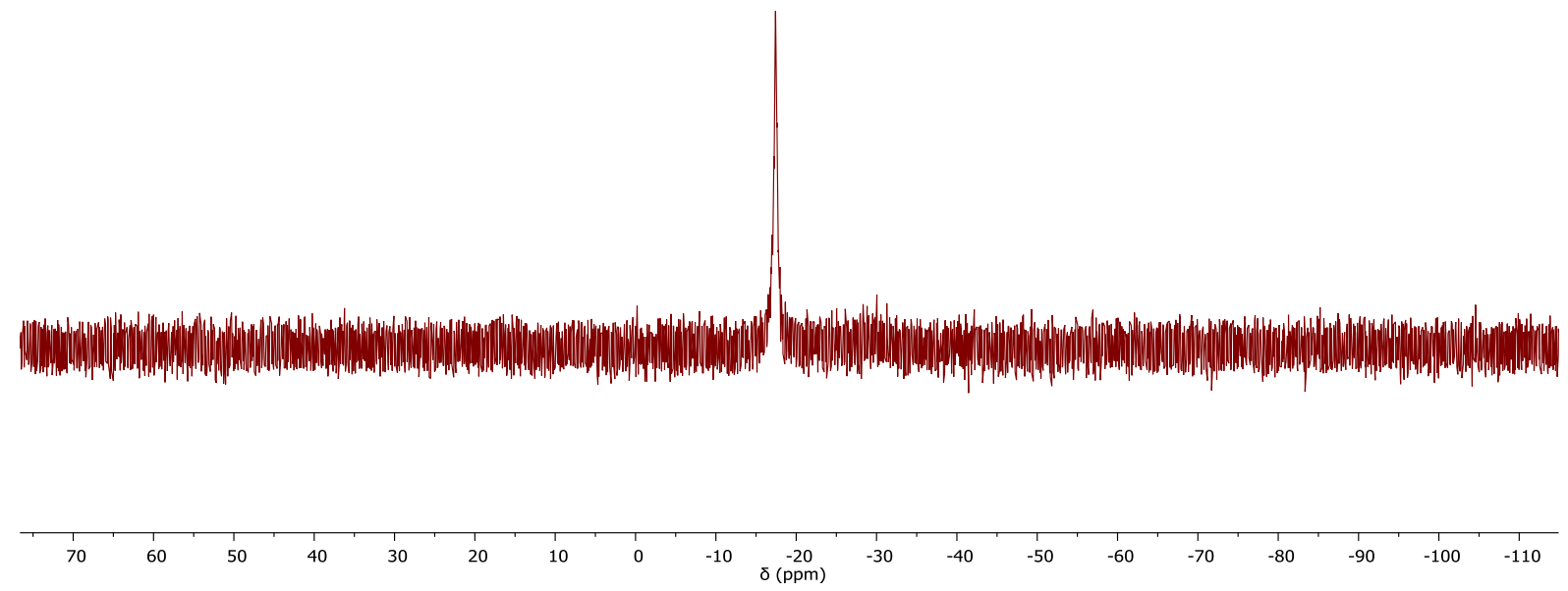

Figure S91. ${ }^{31} \mathrm{P}\left\{{ }^{1} \mathrm{H}\right\}-\mathrm{NMR}\left(\mathrm{THF}-\mathrm{d}_{8}\right)$ of $\left[\mathrm{Ph}_{2} \mathrm{PCHC}(\mathrm{Me}) \mathrm{CH}_{2} \mathrm{Li}(\mathrm{TMEDA})\right] \mathbf{1 1}$

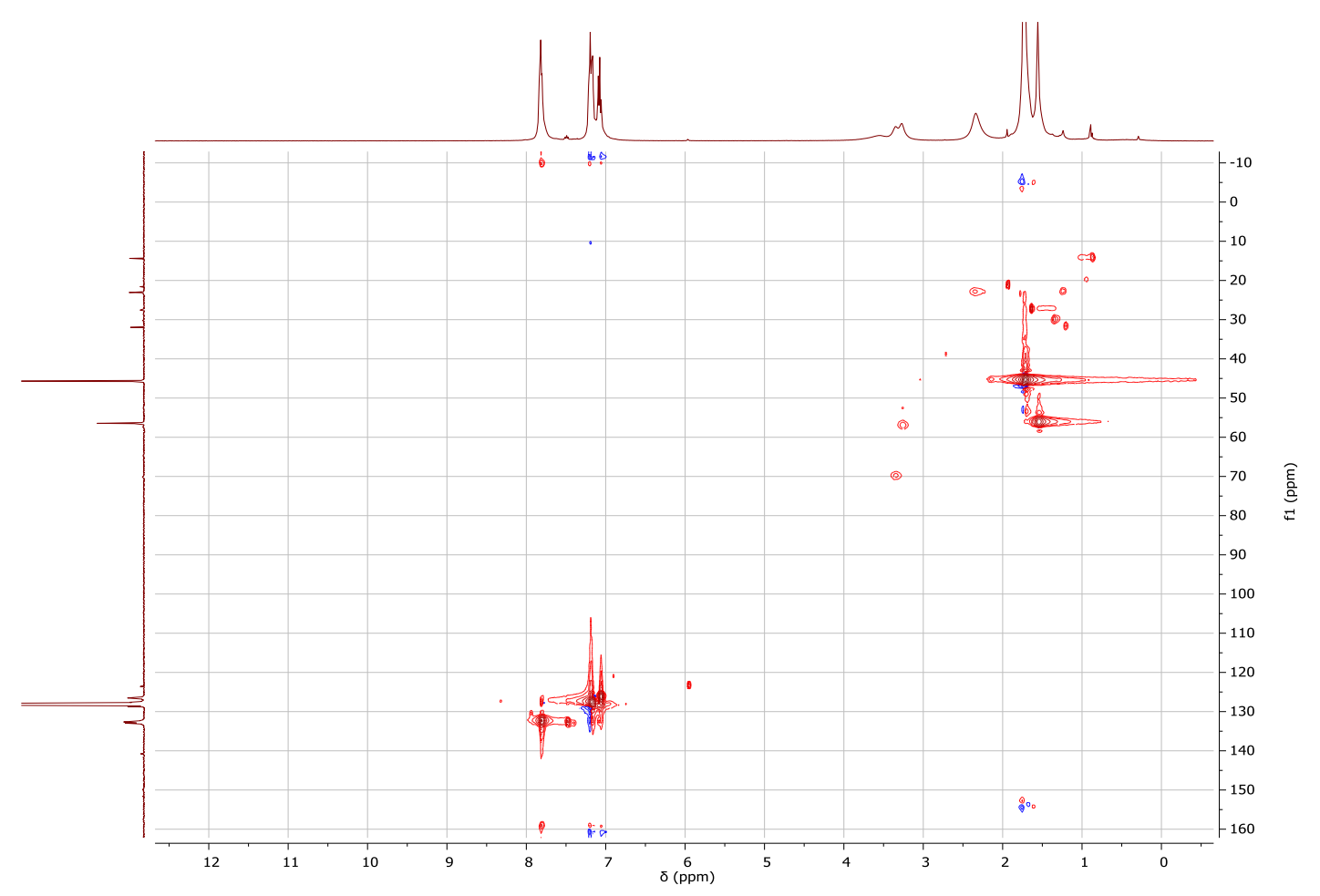

Figure S92. HMQC-NMR $\left(\mathrm{C}_{6} \mathrm{D}_{6}\right)$ of $\left[\mathrm{Ph}_{2} \mathrm{PCHC}(\mathrm{Me}) \mathrm{CH}_{2} \mathrm{Li}(\mathrm{TMEDA})\right] \mathbf{1 1}$ 


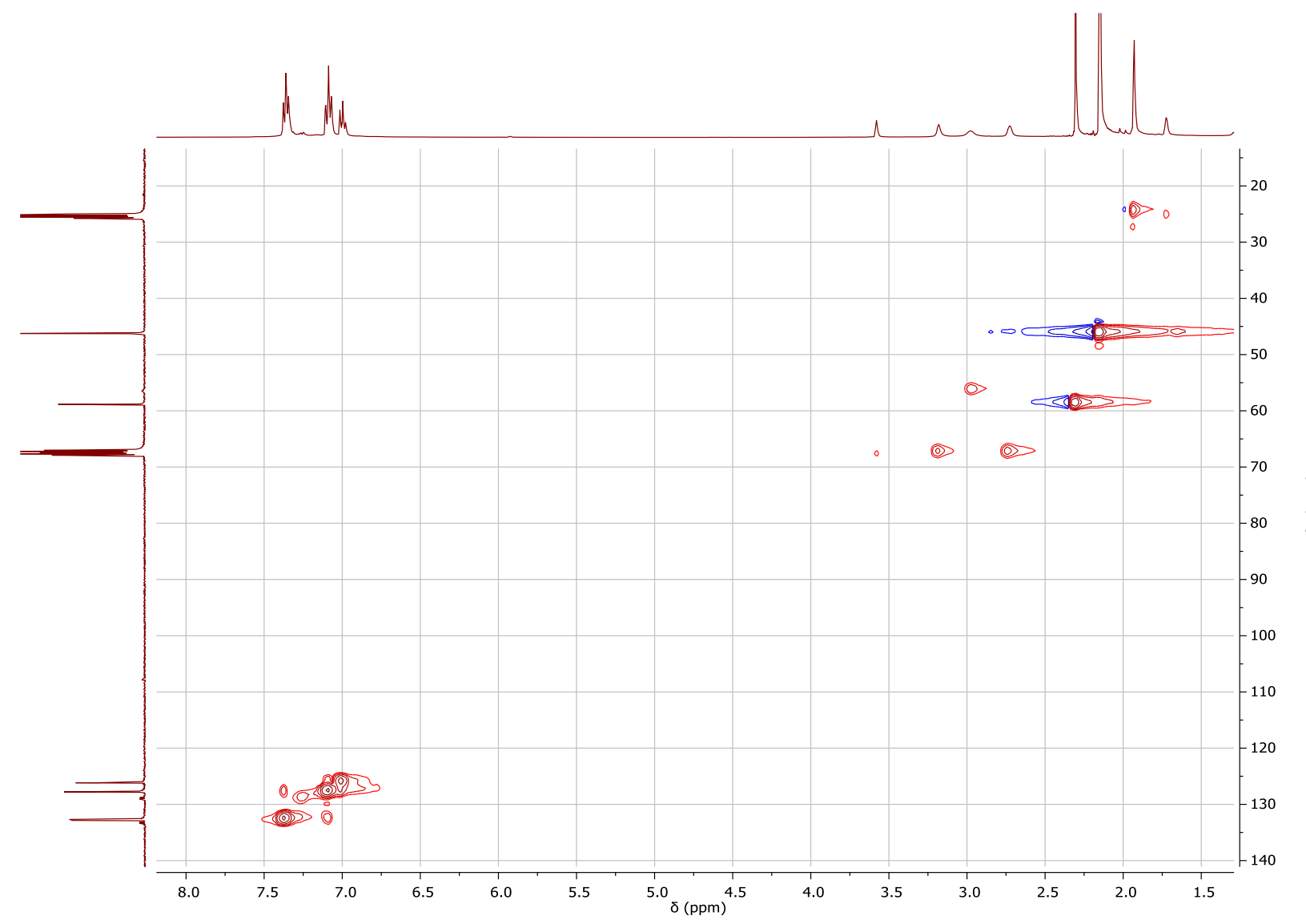

Figure S93. HMQC-NMR (THF-d8) of $\left[\mathrm{Ph}_{2} \mathrm{PCHC}(\mathrm{Me}) \mathrm{CH}_{2} \mathrm{Li}(\mathrm{TMEDA})\right] 11$

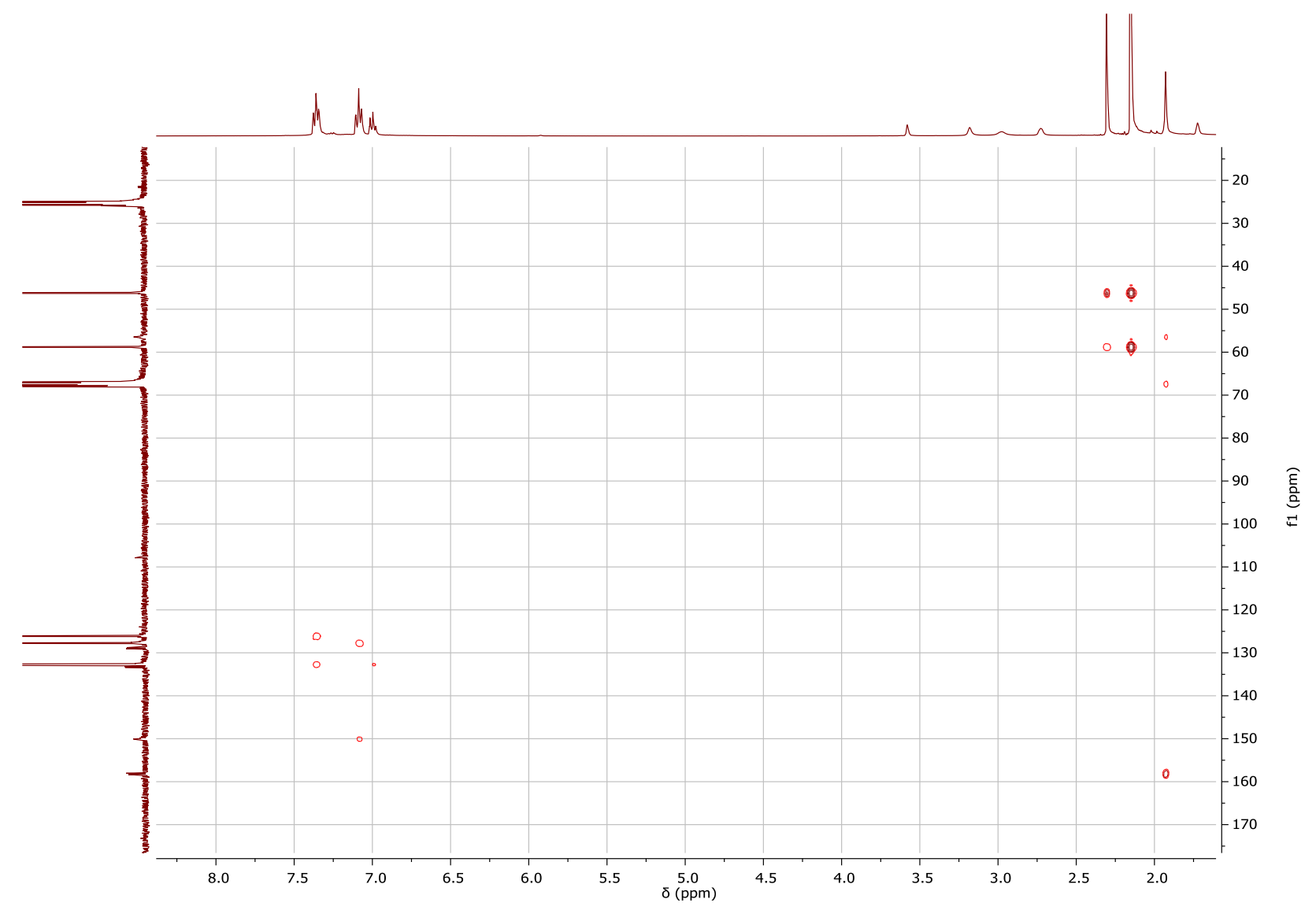

Figure S94. HMBC-NMR (THF- $\left.\mathrm{d}_{8}\right)$ of $\left[\mathrm{Ph}_{2} \mathrm{PCHC}(\mathrm{Me}) \mathrm{CH}_{2} \mathrm{Li}(\mathrm{TMEDA})\right] 11$ 


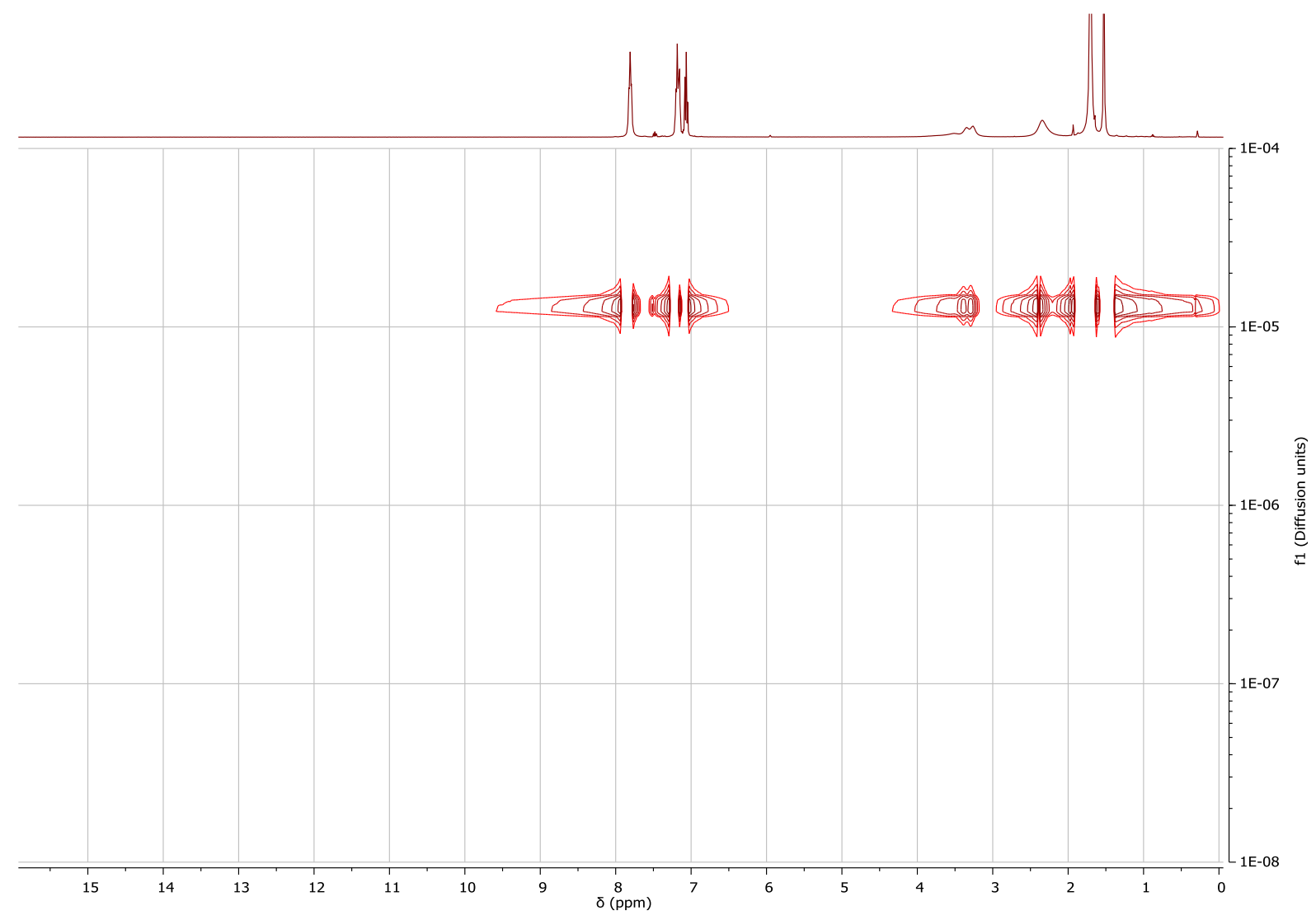

Figure S95. DOSY-NMR $\left(\mathrm{C}_{6} \mathrm{D}_{6}\right)$ of $\left[\mathrm{Ph}_{2} \mathrm{PCHC}(\mathrm{Me}) \mathrm{CH}_{2} \mathrm{Li}(\mathrm{TMEDA})\right] 11$ 


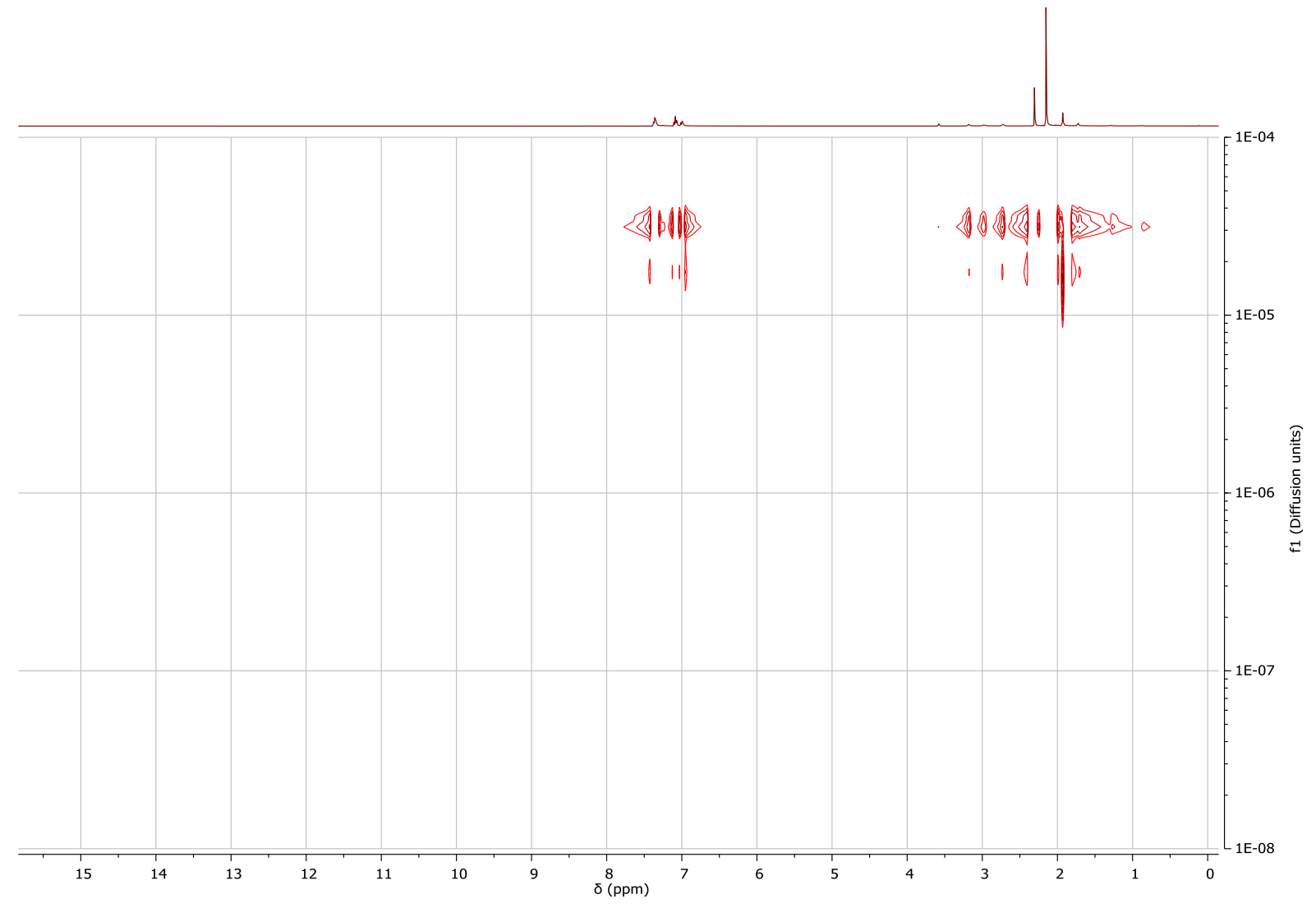

Figure S96. DOSY-NMR (THF-d 8 ) of $\left[\mathrm{Ph}_{2} \mathrm{PCHC}(\mathrm{Me}) \mathrm{CH}_{2} \mathrm{Li}(\mathrm{TMEDA})\right] 11$ 


\section{NMR spectra of $\left[\left\{\mathrm{Ph}_{2} \mathrm{P}(\mathrm{O}) \mathrm{CHCHCH}_{2} \mathrm{Li}_{2}\right\}_{2}\left(\mathrm{Et}_{2} \mathrm{O}\right)_{\mathbf{n}}\right]$ 12:}

${ }^{1} \mathrm{H}$ NMR $\left(\mathrm{C}_{6} \mathrm{D}_{6}, 400.20 \mathrm{MHz}\right): \delta 1.06$ (br-t, 6H, Et $\left.2 \mathrm{O}-\mathrm{CH}_{3}\right), 3.22\left(\mathrm{br}-\mathrm{q}, 4 \mathrm{H}, \mathrm{Et}_{2} \mathrm{O}-\mathrm{CH}_{2}\right), 3.37\left(\mathrm{dd},{ }^{2} J_{\mathrm{H}-\mathrm{P}}=21.0 \mathrm{~Hz},{ }^{3} J_{\mathrm{H}-\mathrm{H}(\beta)}=\right.$ $12.3 \mathrm{~Hz}, 2 \mathrm{H}, \mathrm{P}-\mathrm{CH}-) 3.66\left(\mathrm{dt},{ }^{3} J_{\mathrm{H}-\mathrm{H}(\beta)}=10.1 \mathrm{~Hz},{ }^{3} J_{\mathrm{H}-\mathrm{H}(\text { trans })}=3.5 \mathrm{~Hz}, 2 \mathrm{H},=\mathrm{CHH}(\right.$ cis $\left.)\right), 4.17\left(\mathrm{dd},{ }^{3} J_{\mathrm{H}-\mathrm{H}(\beta)}=16.4 \mathrm{~Hz},{ }^{3} J_{\mathrm{H}-\mathrm{H}(\text { cis })}=\right.$ $2.6 \mathrm{~Hz}, 2 \mathrm{H},=\mathrm{CHH}($ trans $)), 6.63\left(\mathrm{dddd},{ }^{3} J_{\mathrm{H}-\mathrm{P}}=18.1 \mathrm{~Hz},{ }^{3} J_{\mathrm{H}-\mathrm{H}(\text { trans })}=16.5 \mathrm{~Hz},{ }^{3} J_{\mathrm{H}-\mathrm{H}(\alpha)}=12.3 \mathrm{~Hz},{ }^{3} J_{\mathrm{H}-\mathrm{H}(\mathrm{cis})}=10.1 \mathrm{~Hz}, 2 \mathrm{H},-\right.$ $\mathrm{CH}=), 7.03(\mathrm{~m}, 4 \mathrm{H}, p-\mathrm{H}), 7.03(\mathrm{~m}, 2 \mathrm{H}, p-\mathrm{H}), 7.03(\mathrm{~m}, 4 \mathrm{H}, m-\mathrm{H}), 7.91(\mathrm{~m}, 4 \mathrm{H}, o-\mathrm{H})$.

${ }^{7} \mathrm{Li}$ NMR $\left(\mathrm{C}_{6} \mathrm{D}_{6}, 155.5 \mathrm{MHz}\right): \delta-3.67$.

${ }^{13} \mathrm{C}$ NMR $\left(\mathrm{C}_{6} \mathrm{D}_{6}, 100.6 \mathrm{MHz}\right): \delta 15.54\left(\mathrm{~s}, \mathrm{Et}_{2} \mathrm{O}-\mathrm{CH}_{3}\right), 46.49$ (br-m, P-CH-), 65.90 (s, Et $\left.\mathrm{Et}_{2} \mathrm{O}-\mathrm{CH}_{2}\right), 83.66\left(\mathrm{br}-\mathrm{m},=\mathrm{CH}_{2}\right), 128.68$ $\left(\mathrm{d},{ }^{3} J_{\mathrm{C}-\mathrm{P}}=11.6 \mathrm{~Hz}, m-\mathrm{C}\right), 130.84\left(\mathrm{~d},{ }^{4} J_{\mathrm{C}-\mathrm{P}}=2.3 \mathrm{~Hz}, p-\mathrm{C}\right), 133.32\left(\mathrm{~d},{ }^{2} J_{\mathrm{C}-\mathrm{P}}=9.8 \mathrm{~Hz}, o-\mathrm{C}\right), 141.82$ (br m, -CH=).

${ }^{31} \mathrm{P}\left\{{ }^{1} \mathrm{H}\right\}$ NMR $\left(\mathrm{C}_{6} \mathrm{D}_{6}, 162.0 \mathrm{MHz}\right): \delta 38.8$.

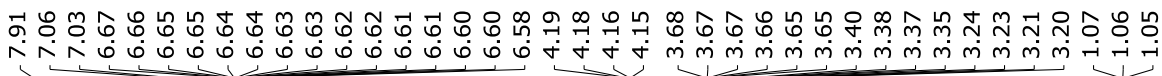

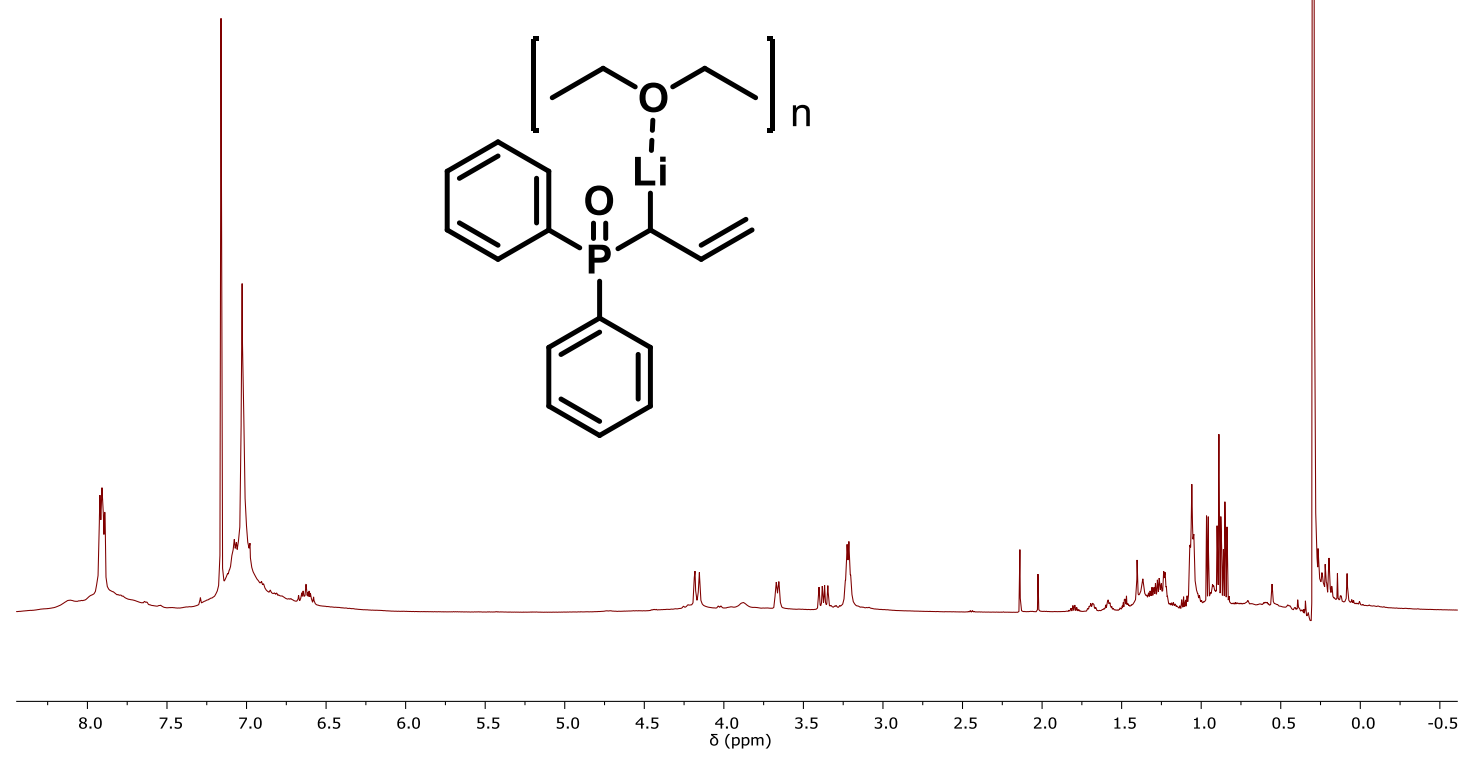

Figure S97. ${ }^{1} \mathrm{H}-\mathrm{NMR}\left(\mathrm{C}_{6} \mathrm{D}_{6}\right)$ of $\left[\left\{\mathrm{Ph}_{2} \mathrm{P}(\mathrm{O}) \mathrm{CHCHCH}_{2} \mathrm{Li}_{2}\left(\mathrm{Et}_{2} \mathrm{O}\right)\right] \mathbf{1 2}\right.$ 

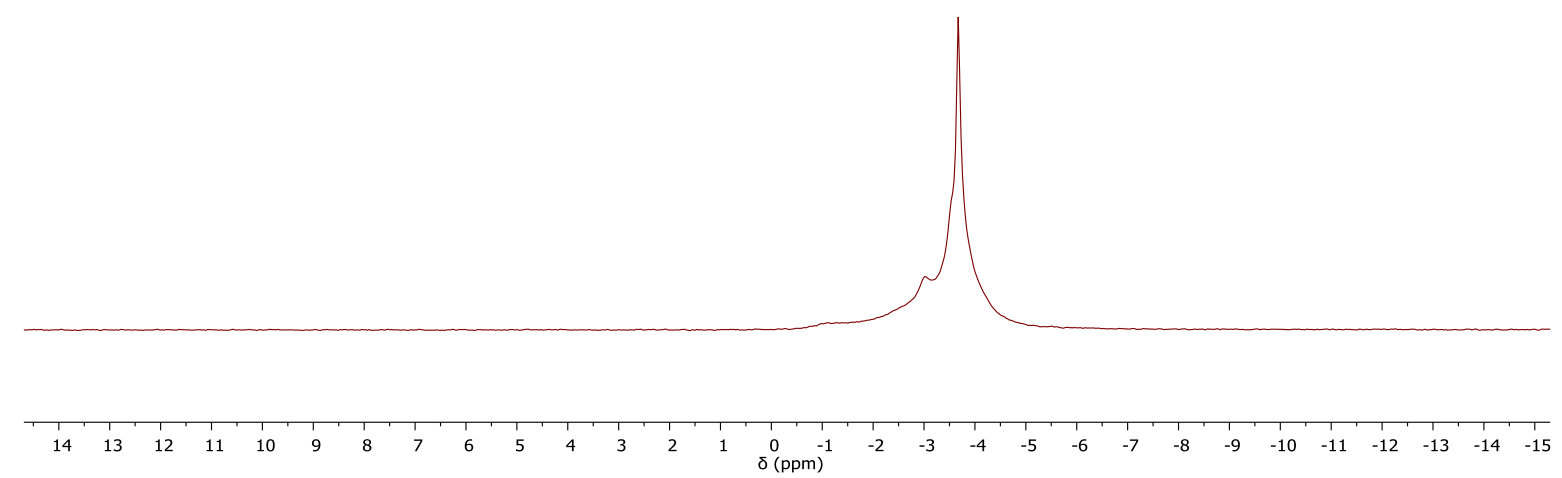

Figure S98. ${ }^{7} \mathrm{Li}-\mathrm{NMR}\left(\mathrm{C}_{6} \mathrm{D}_{6}\right)$ of $\left[\left\{\mathrm{Ph}_{2} \mathrm{P}(\mathrm{O}) \mathrm{CHCHCH}_{2} \mathrm{Li}_{2}\left(\mathrm{Et}_{2} \mathrm{O}\right)\right] 12\right.$

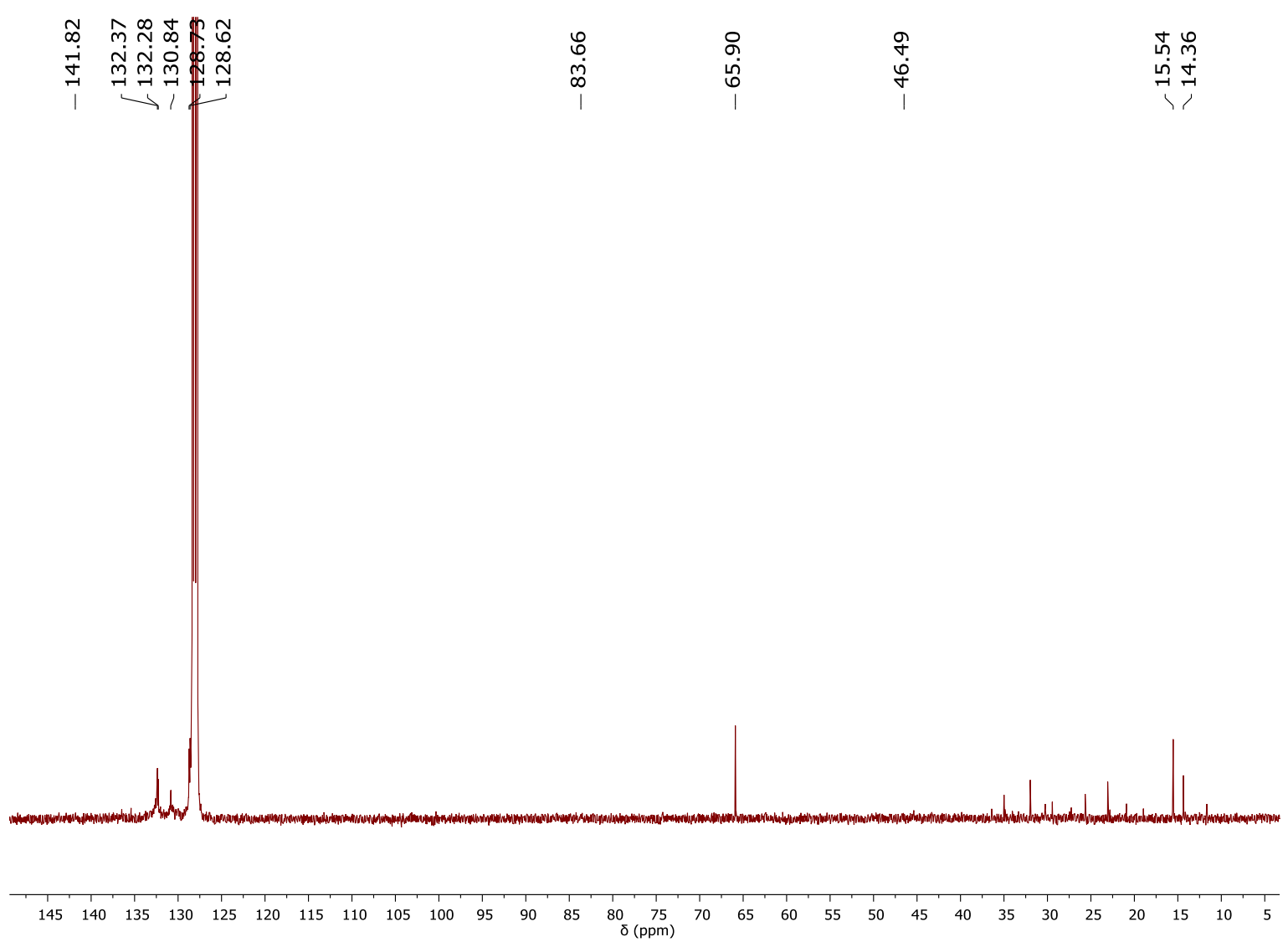

Figure S99. ${ }^{13} \mathrm{C}-\mathrm{NMR}\left(\mathrm{C}_{6} \mathrm{D}_{6}\right)$ of $\left[\left\{\mathrm{Ph}_{2} \mathrm{P}(\mathrm{O}) \mathrm{CHCHCH}_{2} \mathrm{Li}_{2}\left(\mathrm{Et}_{2} \mathrm{O}\right)\right] \mathbf{1 2}\right.$ 


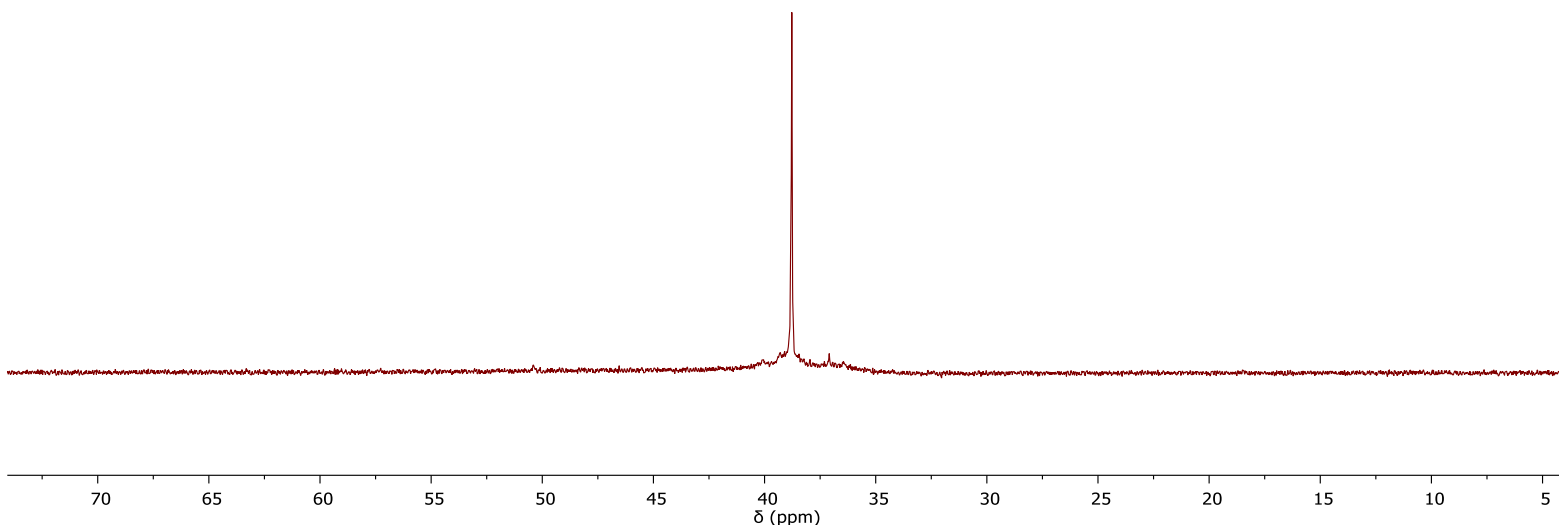

Figure S100. ${ }^{31} \mathrm{P}\left\{{ }^{1} \mathrm{H}\right\}-\mathrm{NMR}\left(\mathrm{C}_{6} \mathrm{D}_{6}\right)$ of $\left[\left\{\mathrm{Ph}_{2} \mathrm{P}(\mathrm{O}) \mathrm{CHCHCH}_{2} \mathrm{Li}_{2}\left(\mathrm{Et}_{2} \mathrm{O}\right)\right] 12\right.$

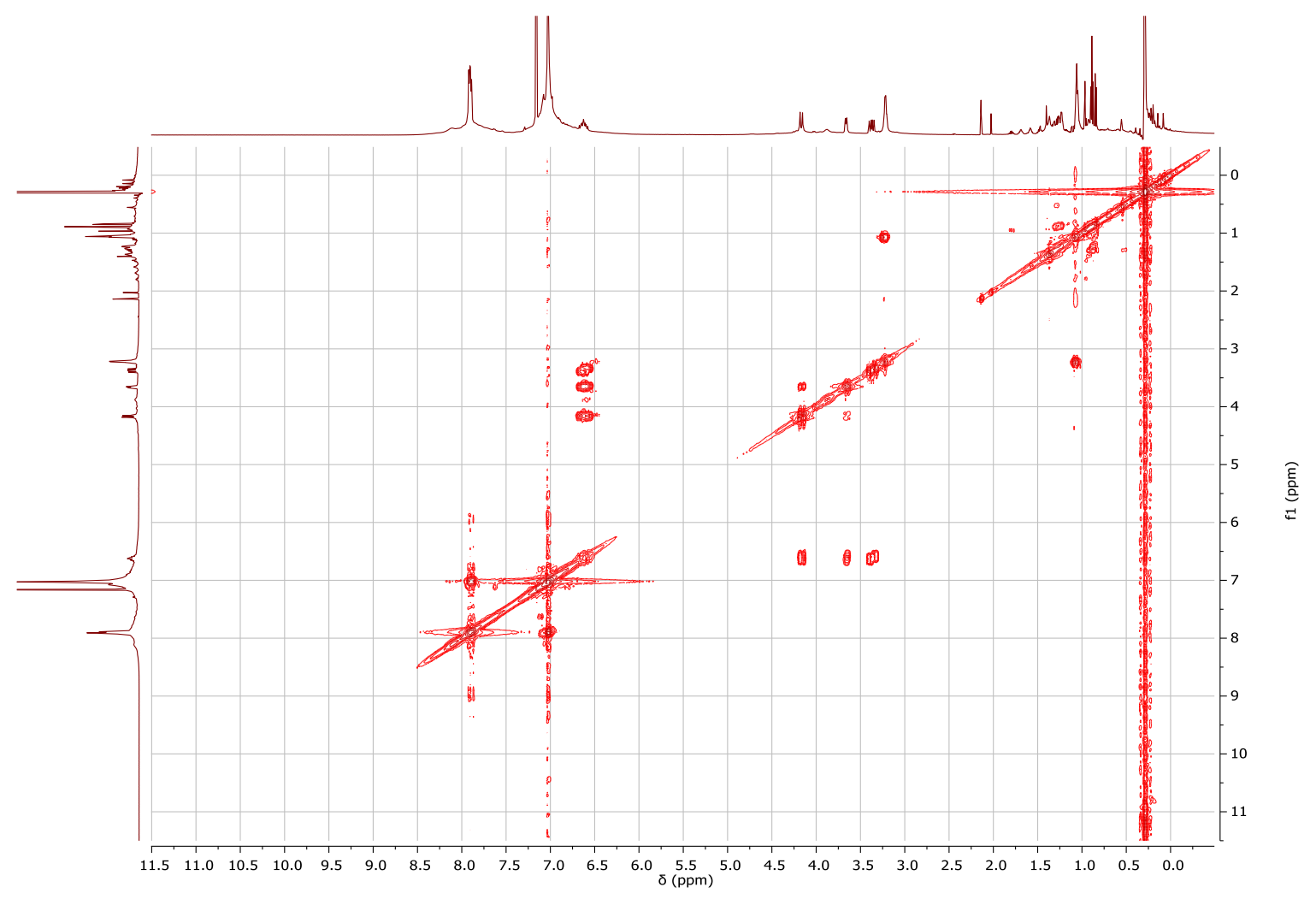

Figure S101. ${ }^{1} \mathrm{H}-{ }^{1} \mathrm{H}$ COSY-NMR $\left(\mathrm{C}_{6} \mathrm{D}_{6}\right)$ of $\left[\left\{\mathrm{Ph}_{2} \mathrm{P}(\mathrm{O}) \mathrm{CHCHCH}_{2} \mathrm{Li}_{2}\left(\mathrm{Et}_{2} \mathrm{O}\right)\right] \mathbf{1 2}\right.$ 


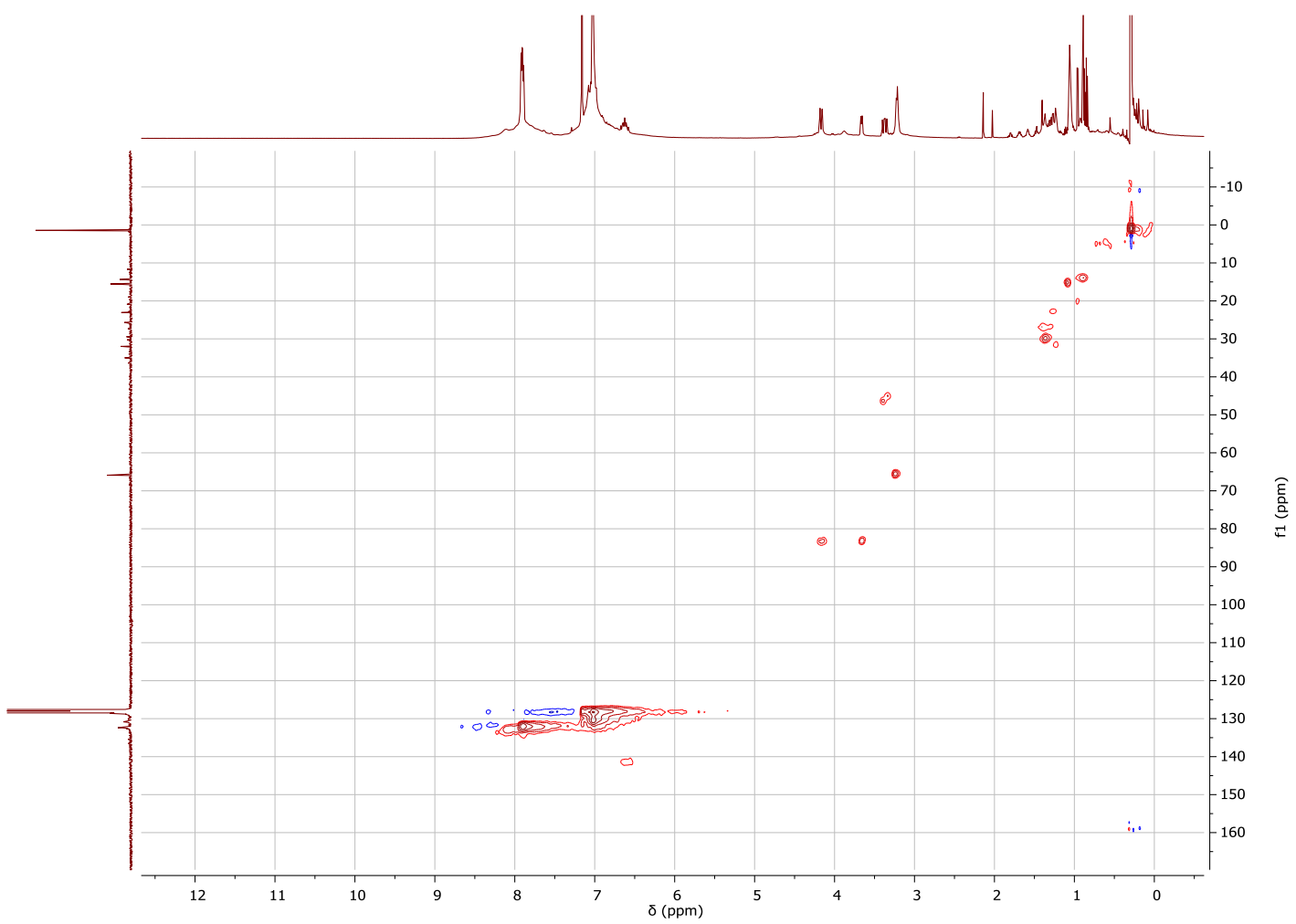

Figure S102. HMQC-NMR $\left(\mathrm{C}_{6} \mathrm{D}_{6}\right)$ of $\left[\left\{\mathrm{Ph}_{2} \mathrm{P}(\mathrm{O}) \mathrm{CHCHCH}_{2} \mathrm{Li}_{2}\left(\mathrm{Et}_{2} \mathrm{O}\right)\right] 12\right.$

S73 